\title{
Spin-orbit interaction in quantum dots and quantum wires of correlated electrons - A way to spintronics?
}

\author{
Dissertation \\ zur Erlangung des Doktorgrades \\ der Mathematisch-Naturwissenschaftlichen Fakultäten \\ der Georg-August-Universität zu Göttingen
}

vorgelegt von

Jens Eiko Birkholz

aus Hannover

Göttingen 2008 
D7

Referent: Prof. Dr. Volker Meden

Korreferent: Prof. Dr. Reiner Kree

Tag der mündlichen Prüfung: 6.10.2008 


\begin{abstract}
We study the influence of the spin-orbit interaction on the electronic transport through quantum dots and quantum wires of correlated electrons. Starting with a one-dimensional infinite continuum model without Coulomb interaction, we analyze the interplay of the spin-orbit interaction, an external magnetic field, and an external potential leading to currents with significant spin-polarization in appropriate parameter regimes. Since lattice models are known to often be superior to continuum models in describing the experimental situation of low-dimensional mesoscopic systems, we construct a lattice model which exhibits the same low-energy physics in terms of energy dispersion and spin expectation values. Confining the lattice to finite length and connecting it to two semi-infinite noninteracting Fermi liquid leads, we calculate the zero temperature linear conductance using the Landauer-Büttiker formalism and show that spin-polarization effects also evolve for the lattice model by adding an adequate potential structure and can be controlled by tuning the overall chemical potential of the system (quantum wire and leads). Next, we allow for a finite Coulomb interaction and use the functional renormalization group (fRG) method to capture correlation effects induced by the Coulomb interaction. The interacting system is thereby transformed into a noninteracting system with renormalized system parameters. For short wires $(\sim 100$ lattice sites), we show that the energy regime in which spin polarization is found is strongly affected by the Coulomb interaction. For long wires ( $>1000$ lattice sites), we find the power-law suppression of the total linear conductance on low energy scales typical for inhomogeneous Luttinger liquids while the degree of spin polarization stays constant. Considering quantum dots which consist of two lattice sites, we observe the well-known Kondo effect and analyze, how the Kondo temperature is affected by the spin-orbit interaction. Moreover, we show how the linear conductance and the spin-polarization can be controlled by tuning the spin-orbit interaction in an Aharonov-Bohm interferometer with a quantum dot in one arm. Finally, an estimation of the magnitude of the spin-orbit interaction in e.g. semiconductor heterojunctions shows that the system parameters used in our simulations are achievable in experiments. Therefore, the theoretical results obtained in this thesis might also be observable experimentally pointing out the relevance for future spintronic applications.
\end{abstract}




\section{Zusammenfassung}

In der vorliegenden Dissertation wird der Einfluss der Spin-Bahn-Wechselwirkung auf den elektronischen Transport durch Quantenpunkte und Quantendrähte korrelierter Elektronen untersucht. Von einem eindimensionalen unendlichen Kontinuumsmodel ohne Coulomb-Wechselwirkung ausgehend wird zunächst das Zusammenspiel zwischen Spin-Bahn-Wechselwirkung, externem Magnetfeld und externer Potentialstruktur diskutiert und gezeigt, dass Ströme mit signifikanter Spin-Polarisation für geeignet gewählte Systemparameter möglich sind. Da Gittermodelle die experimentelle Situation in niedrig-dimensionalen meso-skopischen Systemen häufig besser beschreiben als entsprechende Kontinuumsmodelle, wird ein Gittermodell konstruiert, welches bezüglich Energiedispersion und Spinpolarisation die gleichen Niedrigenergie-Eigenschaften wie das Kontinuumsmodell aufweist. Anschließend wird das Gitter auf eine endliche Länge beschränkt und mit zwei halbunendlichen wechselwirkungsfreien Zuleitungen, die als Fermi-Flüssigkeiten beschrieben werden können, verbunden. Eine Berechnung des linearen Leitwerts im Rahmen des LandauerBüttiker-Formalismus zeigt, dass spinpolarisierte Ströme durch geeignete Parameterwahl auch für das Gittermodell auftreten, wobei der Grad der Spinpolarisation über das chemische Potential des Gesamtsystems gesteuert werden kann. Um die durch die Coulomb-Wechselwirkung induzierten Korrelationseffekte korrekt zu beschreiben, wird die funktionale Renormierungsgruppenmethode (fRG) benutzt. Für kurze Drähte ( 100 Gitterplätze) wird gezeigt, dass die Coulomb-Wechselwirkung den Energiebereich, in dem Spinpolarisation zu beobachten ist, stark modifizieren kann. Für lange Drähte (> 1000 Gitterplätze) beobachtet man eine Unterdrückung des linearen Leitwerts in Form eines Potenzgesetzes auf niedrigen Energieskalen, was charakteristisch für das Verhalten inhomogener Luttinger-Flüssigkeiten ist, wohingegen der Grad der Spinpolarisation erhalten bleibt. Bei der Untersuchung von Quantenpunkten (zwei Gitterplätze) wird der Kondo-Effekt beobachtet und der Einfluss der Spin-Bahn-Wechselwirkung auf die Kondo-Temperatur analysiert. Weiterhin wird für ein Aharonov-Bohm-Interferometer mit einem Quantenpunkt in dem einen Arm gezeigt, dass auch in komplizierteren Netzwerken der lineare Leitwert und die Spin-Polarisation durch Anpassung der Spin-Bahn-Kopplung gesteuert werden können. Eine Betrachtung der Stärke der Spin-Bahn-Kopplung in z.B. HalbleiterHeterostrukturen zeigt, dass die hier gewählten Systemparameter experimentell realisierbar sind. Die in dieser Arbeit theoretisch diskutierten Ergebnisse könnten somit von bedeutender Relevanz für zukünftige Anwendungen in der Spintronik sein. 


\section{Contents}

\begin{tabular}{lll}
\hline & Introduction & 1
\end{tabular}

2 Spin-orbit interaction (SOI) in continuum 4

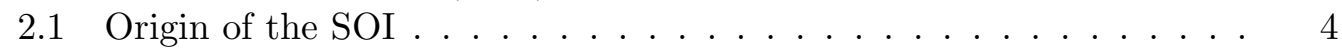

2.21 D continuum model. SOI and Zeeman effect . . . . . . . . . 7

2.3 Transport and spin polarization . . . . . . . . . . . . . . 10

2.3.1 Homogenous svstem. $V_{0}=0$. . . . . . . . . . . 12

2.3 .2 Potential step . . . . . . . . . . . . . . . . . . . . 14

2.3 .3 Potential barrien . . . . . . . . . . . . . . . 16

2.4 Summarv . . . . . . . . . . . . . . . . . . . 21

$\begin{array}{lll}3 & \text { Transport properties of a non-interacting lattice model with SOI } 22\end{array}$

3.1 Lattice model with SOI and Zeeman term . . . . . . . . . . . . . 22

3.2 Preliminary discussion about transport on a lattice . . . . . . . . . . 24

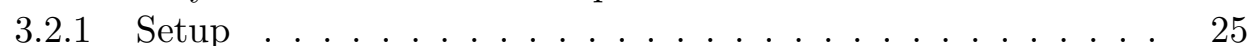

3.2 .2 Projection of the leads . . . . . . . . . . . . . . . . . 27

3.2 .3 The linear conductance . . . . . . . . . . . . . . 28

3.3 The four components of the linear conductance . . . . . . . . . . 29

3.4 Interplav of SOI and magnetic field . . . . . . . . . . . . . . 33

3.4 .1 Potential step . . . . . . . . . . . . . . . . . . . . . . . . . . . . . . 35

3.4 .2 Single impurity . . . . . . . . . . . . . . . . . . . . . . . 38

3.5 Summarv . . . . . . . . . . . . . . . . . . . . . . . 41

4 The functional renormalization group (fRG) 43

4.1 Functional integral and vertex functions . . . . . . . . . . . . . . . . . . 4 44

4.2 Infrared cutoff and flow equations . . . . . . . . . . . . . . . 46

4.3 Numerical realization . . . . . . . . . . . . . . . . . . . . . . . . . . 49

4.4 Summarv $\ldots \ldots \ldots \ldots \ldots \ldots$

5 Quantum wires of correlated electrons with SOI and magnetic field 53

5.1 Transport properties of short quantum wires with SOI, magnetic field and Coulomb interaction . . . . . . . . . . . . . 55

5.1 .1 Potential step . . . . . . . . . . . . . . . . 56

5.1 .2 Potential barrien . . . . . . . . . . . . . . 58 
5.2 Luttinger liauid behavior in long guantum wires . . . . . . . . . 62

5.2.1 Correlated electrons in svstems with single impuritv . . . . . 63

5.2.2 Correlated electrons in svstems with potential step . . . . . . 68

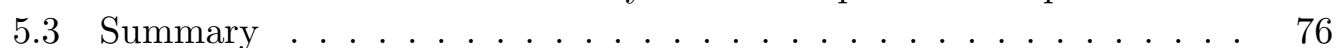

6 Quantum dots of correlated electrons with SOI and magnetic field 79

6.1 Transport through a serial quantum dot with SOI and magnetic field 80

6.1 .1 Non-interacting case . . . . . . . . . . . . . . 80

6.1.2 Coulomb interaction . . . . . . . . . . . . . . 87

6.2 Aharonov-Bohm interferometer . . . . . . . . . . . . . . . . 93

6.2 .1 Non-interacting case . . . . . . . . . . . . . . . . . 94

6.2 .2 Coulomb interaction . . . . . . . . . . . . . . 100

6.3 Summary . . . . . . . . . . . . . . . . . . . 102

\begin{tabular}{lll}
\hline & Towards experimental realization & 104
\end{tabular}

7.1 Realization of quantum dots . . . . . . . . . . . . . . . . . . . . 104

7.2 Realization of quantum wires . . . . . . . . . . . . 106

7.3 The svstem parameters . . . . . . . . . . . . . . . . . . 107

$\begin{array}{lll}8 & \text { Conclusions and outlook } & 109\end{array}$

\begin{tabular}{|ll}
\hline A The Green's function of the leads & 113
\end{tabular}

\begin{tabular}{|ll}
\hline $\mathcal{O}(N)$-algorithm for matrix inversion in presence of SOI & 117
\end{tabular}

B.1 Matrix representation in presence of SOI . . . . . . . . . . . . 117

B.2 Calculation of the $G_{1, N}$ matrix element of the full propagator . . . 120

\begin{tabular}{|l|l|l|l|}
\hline$C$ & The eigenvalue problem of the double dot & 122
\end{tabular}

\begin{tabular}{lr}
\hline Bibliography & 125
\end{tabular}

\begin{tabular}{lr}
\hline Acknowledgement & 130
\end{tabular}

\begin{tabular}{lr}
\hline Lebenslauf & 131
\end{tabular} 


\section{Chapter 1}

\section{Introduction}

Modern microelectronics has undergone a matchless development in the past 20 years. The emerging field of spintronics is often believed to continue this story of success. The birth of spintronics is dated to 1988 with the discovery of the giant magnetoresistance effect 1, 2]. Since then, many theoretical and experimental studies have been performed on spin-dependent electronic transport in order to get a clear understanding of the underlying physics and to investigate the possibility of fabricating spintronic devices 3, 4, 5]. In general, the term spintronics can be understood as spin-based electronics in which not only the electron charge, but also the electron spin carries information. This offers the opportunity of creating a new generation of devices by implementing spin-dependent effects into standard microelectronics [6].

Quantum dots and quantum wires with spin-orbit interaction are very well suited examples of physical systems which may provide a good starting point for a spintronic device setup. These low-dimensional electron systems are strongly affected by the two-particle Coulomb interaction making an exact theoretical description difficult or even impossible. However, during roughly the same period in which the fabrication of such well-defined low-dimensional systems has become possible due to the fast development of vacuum deposition and epitaxial growth technology on the one hand and elaborate measurement techniques for a precise investigation of the physical properties of these systems on the other hand, many theoretical tools and methods have been developed to tackle the influence of the Coulomb interaction on the low-energy behavior of interacting low-dimensional electron systems.

In this thesis, the recently developed functional renormalization group method (fRG) is used to estimate and predict correlation effects due to Coulomb interaction in electron systems with spin-orbit interaction. The fRG is based on Wilson's general RG idea [7] and starts with the introduction of an infrared cutoff to the free propagator of the system under consideration, therefore neglecting all modes with energy below this cutoff. Derivation with respect to this cutoff parameter leads to an exact hierarchy of flow equations for the one-particle irreducible vertex functions, which are obtained from a generating functional by derivation with respect 
to external source fields. In practice, this hierarchy of flow equations needs to be truncated in order to be integrated numerically [8, 9. Since the numercial effort of the fRG within our approach is quite small compared to other RG methods such as the numerical renormalization group (NRG) 7, 10, or the density-matrix renormalization group (DMRG) method [11, 12, 13, very large systems of up to $10^{6}$ lattice sites can be addressed with this method in the case of spinless fermions (Hubbard model) and up to $10^{4}$ lattice sites for systems of spinful fermions (extended Hubbard model). Systems of this size with impurities are known to show characteristics of inhomogeneous Luttinger liquids, namely a power-law behavior of correlation functions over some energy range with interaction-dependent exponents [14. One example for the great success of the fRG in the last few years is the prediction of the correct exponents in leading order of the Coulomb interaction strength compared to the analytical solution which is possible by Bethe ansatz for special integrable cases [15, 16, 17, 18. Moreover, fRG accounts for aspects of correlation effects in quantum dots, e.g. the Kondo effect [19, 20]. It will be shown that the fRG method is also the appropriate computational tool for investigating the low-energy behavior of zero- and one-dimensional quantum systems with spin-orbit interaction.

The spin-orbit interaction (SOI) is a relativistic effect of order $\mathcal{O}\left(v^{2} / c^{2}\right)$, which follows directly from the Dirac equation by expansion in $v / c$ and is known to be responsible for many interesting quantum effects affecting the electron spin in systems with large gradients of the electrostatic potential, e.g. heavy atoms or semiconductor heterostructures [21. The latter define a two-dimensional electron gas (2DEG) which can be confined further by applying a structure of gate electrodes to form a one- or even zero-dimensional electron system. Due to this sharp confinement, any classical or semi-classical description of the electrons breaks down and quantum mechnical properties like the electron spin must be taken into consideration.

It has recently be shown by conductance measurements and excited-state spectroscopy that carbon nanotubes which are known of being well-described by onedimensional systems of correlated electrons reveal a remarkable SOI [22]. Besides their outstanding mechanical properties and the possibility of applying electrical gates to the carbon nanotubes as well as connecting them to higher-dimensional leads, the occurence of SOI makes carbon nanotubes being a prominent candidate for spintronic applications.

Another realization of a physical system with considerable SOI is a quantum dot formed by applying gate electrodes to a two-dimensional electron gas (2DEG) at the interface of a semiconductor hetero-structure. GaAs quantum dots reveal prodigious SOI induced effects as has been shown by measurements and theoretical analysis of conductance oscillations [23, 24. The SOI in GaAs quantum dots is caused by the intrinsic asymmetry of the underlying atomic Zinkblende structure of GaAs on the one hand and the sharp gradients of the ambient scalar potential confining the electrons on the other hand. The resulting electric fields and therefore the SOI can be tuned by an external gate voltage. Hence, one may hope to be able to control the electron spin by controlling the strength of the SOI.

This thesis is organized as follows. In Chapter 2 we give a short derivation of the 
SOI Hamiltonian for an infinite one-dimensional continuum model and discuss the low-energy properties such as energy dispersion and spin expectation values of the lowest energy subband. The interplay of SOI and an arbitrary external magnetic field will be shown to lead to subtle spin polarization effects considering the electronic transport across a potential step and a potential barrier.

Chapter [3 starts with the construction of a tight-binding lattice model which exhibits the same low-energy physics as the continuum model in terms of energy dispersion and spin expectation values. We examine a lattice system of finite length being attached to two electron reservoirs which can be regarded as Fermi liquids. This setup matches the experimental situation often better than the infinite continuum model. We study the linear conductance at zero temperature $(T=0)$ and the spin polarization of this system in presence of a potential step and a potential barrier the latter of which can be thought of an impurity atom, thus meeting quite a realistic experimental situation.

The functional renormalization group method which will be proved to be a suitable numerical tool to tackle the Coulomb interaction in electron systems with SOI is presented in Chapter 4

Chapter $[$ is the sequel to Chapter 3 but with focus on the finite Coulomb interaction and its influence on the linear conductance and the spin polarization. Luttinger liquid behavior will manifest itself in these systems and the influence of SOI on the power law decay of correlation functions, like the linear conductance, will be investigated. Moreover, we will explore the asymptotic behavior of the spin polarization.

In Chapter [6] we study a very short lattice system of only two sites representing a two-level quantum dot in the spin-degenerate case. Again, the system under consideration is coupled to two higher-dimensional leads. A detailed analysis of the influence of SOI, magnetic field and Coulomb interaction on the energy level splitting and on the linear conductance and the spin polarization will be given. Especially, we will analyse the impact of the SOI on the Kondo effect and its relevant energy scale, the Kondo temperature. Furthermore, we will study interference effects in an Aharonov-Bohm interferometer with the double quantum dot of correlated electrons in one arm and a spin-dependent hopping in the other.

Chapter 7 contains a short description of recent experiments matching the different situations described theoretically in this thesis. We will give a profound estimation of the parameters used in our model taking into account data from abinitio calculations as well as from experiments. Whenever the reader feels to get lost in technical details, he/she may read through this chapter to hold up a clear view of the motivation behind our calculations.

Finally in Chapter 8 we will sum up the results obtained in this thesis and give an outlook of further investigations which could be performed with the methods presented here. Furthermore, we will shortly address additional effects that have been omitted in our models. 


\section{Chapter 2}

\section{Spin-orbit interaction (SOI) in continuum}

In this chapter, we address the spin-orbit interaction (SOI) as a relativistic effect following directly from the Dirac equation in second order $\mathcal{O}\left(v^{2} / c^{2}\right)$ and investigate its influence on the energy eigenvalues and spin expectation values of a homogeneous one-dimensional continuum model. Starting from the general three-dimensional case, in which the SOI Hamiltonian (in SI-units) can be written as

$$
H_{\mathrm{SO}}=-\frac{e \hbar}{4 m^{2} c^{2}} \boldsymbol{\sigma} \cdot\left[\mathbf{E} \times\left(\mathbf{p}-\frac{e}{c} \mathbf{A}\right)\right],
$$

with the electric field $\mathbf{E}=-\nabla V / e$ ( $e<0$ is the electron charge) being the gradient of an ambient scalar potential $V$, we will confine the system to one dimension and discuss the effect of the SOI.

\subsection{Origin of the SOI}

Before we discuss the effect of SOI on the physical properties of a homogeneous continuum model, we first derive the Hamiltonian Eq. (2.1) from the Dirac equation by an expansion in $v / c$.

The Dirac equation for a particle with mass $m$, charge $e$ and canonical momentum p reads for time-independent problems 25]

$$
\left(c \boldsymbol{\alpha}\left(\mathbf{p}-\frac{e}{c} \mathbf{A}\right)+\beta m c^{2}+V \mathbf{1}_{4}\right) \psi=E \psi
$$

with arbitrary scalar potential $V$, vector potential $\mathbf{A}$ and

$$
\boldsymbol{\alpha}=\left(\begin{array}{ll}
0 & \boldsymbol{\sigma} \\
\boldsymbol{\sigma} & 0
\end{array}\right) \quad \text { and } \beta=\left(\begin{array}{cc}
\mathbf{1}_{2} & 0 \\
0 & -\mathbf{1}_{2}
\end{array}\right)
$$

$\boldsymbol{\sigma}$ is the three-component vector consisting of the Pauli spin matrices which are given by

$$
\sigma_{x}=\left(\begin{array}{cc}
0 & 1 \\
1 & 0
\end{array}\right), \quad \sigma_{y}=\left(\begin{array}{cc}
0 & -i \\
i & 0
\end{array}\right), \quad \sigma_{z}=\left(\begin{array}{cc}
1 & 0 \\
0 & -1
\end{array}\right)
$$


for spin quantization along the $z$-direction. In the following, we omit writing down the $n$-dimensional identity matrices $\mathbf{1}_{n}$ explicitely in favor of a brief notation. $\psi$ denotes a four-component spinor and can be split into an upper and a lower twocomponent part, $\psi=\left(\psi_{A}, \psi_{B}\right)^{T}$. In this notation, we obtain the two coupled equations

$$
\begin{aligned}
\boldsymbol{\sigma}\left(\mathbf{p}-\frac{e}{c} \mathbf{A}\right) \psi_{B} & =\frac{1}{c}(\tilde{E}-V) \psi_{A} \\
\boldsymbol{\sigma}\left(\mathbf{p}-\frac{e}{c} \mathbf{A}\right) \psi_{A} & =\frac{1}{c}\left(\tilde{E}-V+2 m c^{2}\right) \psi_{B}
\end{aligned}
$$

with $\tilde{E}=E-m c^{2}$. This can be transformed into an eigenvalue equation for the upper component $\psi_{A}$,

$$
\boldsymbol{\sigma}\left(\mathbf{p}-\frac{e}{c} \mathbf{A}\right) c^{2}\left(\tilde{E}-V+2 m c^{2}\right)^{-1} \boldsymbol{\sigma}\left(\mathbf{p}-\frac{e}{c} \mathbf{A}\right) \psi_{A}=(\tilde{E}-V) \psi_{A} .
$$

Since the inverse of a diagonal matrix is also diagonal, we can expand $\left(\tilde{E}-V+2 m c^{2}\right)^{-1}$ in the non-relativistic limit, $(\tilde{E}-V) / 2 m c^{2} \ll 1$, into

$$
c^{2}\left(\tilde{E}-V+2 m c^{2}\right)^{-1}=\frac{1}{2 m}\left[1-\frac{\tilde{E}-V}{2 m c^{2}}+\mathcal{O}\left(\frac{v^{3}}{c^{3}}\right)\right]
$$

and obtain the approximate eigenvalue equation

$$
H_{A} \psi_{A}=\left[\boldsymbol{\sigma}\left(\mathbf{p}-\frac{e}{c} \mathbf{A}\right) \frac{1}{2 m}\left(1-\frac{\tilde{E}-V}{2 m c^{2}}\right) \boldsymbol{\sigma}\left(\mathbf{p}-\frac{e}{c} \mathbf{A}\right)+V\right] \psi_{A}=\tilde{E} \psi_{A} .
$$

However, this equation contains several problems. Firstly, the Hamiltonian $H_{A}$ contains the eigenenergy $\tilde{E}$ and is not a Hermitian operator. Secondly, one should note that $\psi_{A}$ itself is not normalized to unity, but $\int d^{3} r \psi^{\dagger} \psi=\int d^{3} r\left(\psi_{A}^{\dagger} \psi_{A}+\right.$ $\left.\psi_{B}^{\dagger} \psi_{B}\right)=1$. Therefore, one defines a new two-component wave function $\psi$ via

$$
\tilde{\psi}=\Omega \psi_{A}=\left[\mathbf{1}_{2}+\frac{\boldsymbol{\sigma}\left(\mathbf{p}-\frac{e}{c} \mathbf{A}\right)}{8 m^{2} c^{2}}\right] \psi_{A},
$$

which is normalized up to $\mathcal{O}\left(v^{2} / c^{2}\right)$. Thus, the new eigenvalue equation for the normalized wave function $\tilde{\psi}$ reads

$$
\Omega^{-1} H_{A} \psi_{A}=\Omega^{-1} H_{A} \Omega^{-1} \tilde{\psi}=\tilde{E}\left(\Omega^{-1}\right)^{2} \tilde{\psi}
$$

with $\Omega^{-1}$ given by

$$
\Omega^{-1}=\mathbf{1}_{2}-\frac{\left[\boldsymbol{\sigma}\left(\mathbf{p}-\frac{e}{c} \mathbf{A}\right)\right]^{2}}{8 m^{2} c^{2}}+\mathcal{O}\left(\frac{v^{3}}{c^{3}}\right)=\mathbf{1}_{2}-\frac{\left(\mathbf{p}-\frac{e}{c} \mathbf{A}\right)^{2}}{8 m^{2} c^{2}}+\frac{e \hbar \boldsymbol{\sigma} \mathbf{B}}{8 m^{2} c^{3}}+\mathcal{O}\left(\frac{v^{3}}{c^{3}}\right)
$$


with the magnetic field $\mathbf{B}=\nabla \times \mathbf{A}$.

In the following, we use the Lorenz gauge, $\nabla \mathbf{A}=0$ (for time-independent scalar potential $V$ ). Assuming that the second partial derivatives of $\mathbf{A}$ commute, $\partial_{i} \partial_{j} \mathbf{A}=$ $\partial_{j} \partial_{i} \mathbf{A}$ with $i, j=x, y, z$, we also get $\nabla \mathbf{B}=0$. Writing the gradient of the scalar potential as an electric field, i.e. $\mathbf{p} V=i e \hbar \mathbf{E}$, we can transform $\Omega^{-1} H_{A} \Omega^{-1}$ in Eq. (2.10) into

$$
\begin{aligned}
& \Omega^{-1} H_{A} \Omega^{-1}=V+\frac{2 m c^{2}-\tilde{E}}{4 m^{2} c^{2}}\left[\left(\mathbf{p}-\frac{e}{c} \mathbf{A}\right)^{2}-\frac{e \hbar}{c} \boldsymbol{\sigma} \mathbf{B}\right]-\frac{e \hbar}{4 m^{2} c^{2}}\left[\mathbf{E} \times\left(\mathbf{p}-\frac{e}{c} \mathbf{A}\right)\right] \boldsymbol{\sigma} \\
& +\frac{e \hbar}{4 m^{3} c^{3}} \underline{\mathbf{p}(\boldsymbol{\sigma} \mathbf{B})} \mathbf{p}-\frac{e \hbar^{2} \operatorname{div} \mathbf{E}}{8 m^{2} c^{2}}-\frac{\left(\mathbf{p}-\frac{e}{c} \mathbf{A}\right)^{4}}{8 m^{3} c^{2}}
\end{aligned}
$$

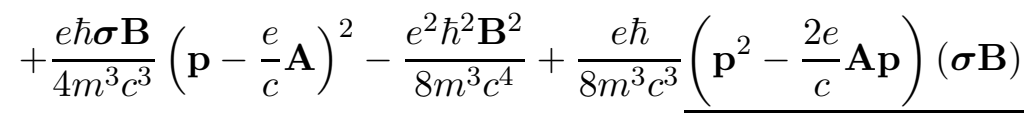

where the underscore indicates that the momentum operator just acts on the underscored part and not on the subsequent wave function. Note that, despite the fact that $\mathbf{p B}=0, \mathbf{p}(\boldsymbol{\sigma} \mathbf{B}) \neq 0$ in general.

Using

$$
\left(\Omega^{-1}\right)^{2}=1-\frac{\left(\mathbf{p}-\frac{e}{c} \mathbf{A}\right)^{2}}{4 m^{2} c^{2}}+\frac{e \hbar \boldsymbol{\sigma} \mathbf{B}}{4 m^{2} c^{3}}+\mathcal{O}\left(\frac{v^{3}}{c^{3}}\right),
$$

we finally can write down the so-called Pauli equation

$$
\tilde{H} \tilde{\psi}=\tilde{E} \tilde{\psi}
$$

with

$$
\begin{aligned}
\tilde{H}= & \frac{\left(\mathbf{p}-\frac{e}{c} \mathbf{A}\right)^{2}}{2 m}+V-\frac{e \hbar}{4 m^{2} c^{2}} \boldsymbol{\sigma}\left[\mathbf{E} \times\left(\mathbf{p}-\frac{e}{c} \mathbf{A}\right)\right]-\frac{e \hbar}{2 m c} \boldsymbol{\sigma} \mathbf{B} \\
& -\frac{\left(\mathbf{p}-\frac{e}{c} \mathbf{A}\right)^{4}}{8 m^{3} c^{2}}+\frac{e \hbar}{8 m^{3} c^{3}}\left(\mathbf{p}-\frac{e}{c} \mathbf{A}\right)^{2}(\boldsymbol{\sigma} \mathbf{B})+\frac{e \hbar}{8 m^{3} c^{3}}(\boldsymbol{\sigma} \mathbf{B})\left(\mathbf{p}-\frac{e}{c} \mathbf{A}\right)^{2} \\
& -\frac{e^{2} \hbar^{2} \mathbf{B}^{2}}{8 m^{3} c^{4}}-\frac{e \hbar^{2} \operatorname{div} \mathbf{E}}{8 m^{2} c^{2}} .
\end{aligned}
$$

In the following, we only take the first line of Eq. (2.15) into account, where we abandon the correction $-e \mathbf{A} / c$ to the canonical momentum, which is a valid approximation in the limit of a weak magnetic field leading to a magnetic length larger than the width of the ambient confining potential. The first and second term are the kinetic and potential energy, respectively, the third term is the spin-orbit interaction and the fourth is the Zeeman term. The higher order corrections to the Zeeman term and the kinetic energy in the second and third line of Eq. (2.15) will be omitted. The last term in the third line is known as the Darwin term, which is responsible for the breakdown of exact localization, i.e. the position of the particle under consideration fluctuates in an area given by the Compton wave length. This effect will not be taken into account either. 
In the next subsection, we will investigate the influence of the spin-orbit interaction on the energy dispersion, eigenvectors and spin expectation values of a homogenous one-dimensional continuum model. The interplay between the SOI and the Zeeman effect will be of particular interest.

\section{$2.21 \mathrm{D}$ continuum model, SOI and Zeeman effect}

The transport properties of non-interacting electrons in quasi one-dimensional quantum wires with SOI and Zeeman effect have already been studied in Refs. [26, 27, 28, 29, 30, 31, 32, but mostly under a very particular aspect and strong restrictions to the system parameters. Therefore, we give a more general overview of the interplay of the SOI and the Zeeman effect.

Starting from a three-dimensional (3D) system of free electrons, one can create a well-defined 1D electron system (in $x$-direction) by considering a confining scalar potential $V$ with large gradients (in $y$ - and $z$-direction). Potentials which confine the electrons at least in one direction occur naturally at the interface of semiconductor heterojunctions, e.g. GaAs/AlGaAs or GaAs/InGaAs [21, and form a two-dimensional electron gas. The confinement in the second direction orthogonal to the first is normally achieved by applying an electrode structure to the 2DEG such that the strengh of the lateral confinement can be tuned very accurately. Another example of experimentally feasible 1D electron systems are carbon nanotubes, which are of particular interest for modern applications due to their excellent mechanical and electrical properties [33].

Considering the electron motion to be possible only in the $x$-direction, i.e. $\mathbf{p}=$ $\left(p_{x}, 0,0\right)$, we obtain from Eq. (2.15) the spin-orbit Hamiltonian

$$
H_{\mathrm{SO}}^{1 \mathrm{D}}=-\frac{e \hbar}{4 m^{2} c^{2}}\left(\sigma_{y} E_{z}-\sigma_{z} E_{y}\right) p_{x}=\frac{\hbar \alpha_{y}}{m} \sigma_{z} p_{x}-\frac{\hbar \alpha_{z}}{m} \sigma_{y} p_{x}
$$

with the SOI coupling parameters

$$
\alpha_{y}=\frac{4 m c^{2}}{e} E_{y} \quad \text { and } \quad \alpha_{z}=\frac{4 m c^{2}}{e} E_{z},
$$

which are proportional to the electric field induced by the gradient of the surrounding scalar potential $V . \alpha_{y}$ and $\alpha_{z}$ are assumed to be constant in space, i.e. on the $x$-axis in our model. The Hamiltonian Eq. (2.16) is often referred to as Rashba Hamiltonian (see e.g. 34]).

With the above-mentioned restrictions to Eq. (2.15), the Hamiltonian for the 1D continuum model with SOI and Zeeman term is given by

$$
H=\frac{p_{x}^{2}}{2 m}-\frac{\hbar \alpha_{z}}{m} \sigma_{y} p_{x}+\frac{\hbar \alpha_{y}}{m} \sigma_{z} p_{x}-\frac{g e \hbar}{4 m_{0} c} \boldsymbol{\sigma} \cdot \mathbf{B},
$$

where we account for neglected effects by considering $m$ as an effective electron mass 35]. Compared to Eq. (2.15), where we implicitely assumed a Landé factor $g=2$, we 
modified the expression of the Zeeman term with $m_{0}$ being the electron mass in vacuum and $g$ being the effective Landé factor. The parameters $m$ and $g$ both strongly depend on the details of the experimental setup like the choice of the material and the precise form of the confinement structure [21]. In contrast to most cases found in the literature, we allow for a magnetic field $\mathbf{B}=B(\sin \theta \cos \varphi, \sin \theta \sin \varphi, \cos \theta)$, pointing in arbitrary direction. The normalized eigenstates are characterized by quantum numbers $k$ and $s= \pm$ and are given by the product of a plane wave (in $x$-direction) and a two-component spinor

$$
\phi_{k}^{(s)}(x)=\frac{1}{\sqrt{2 \pi}} e^{i k x}\left(\begin{array}{c}
A_{k}^{(s)} \\
B_{k}^{(s)}
\end{array}\right)
$$

Applying the Hamiltonian Eq. (2.18) to this ansatz, we obtain

$$
\left(\begin{array}{cc}
k^{2}+2 \alpha_{y} k+2 k_{Z}^{2} \cos \theta-\epsilon, & 2 i k \alpha_{z}+2 k_{Z}^{2} e^{-i \varphi} \sin \theta \\
2 i k \alpha_{z}+2 k_{Z}^{2} e^{i \varphi} \sin \theta, & k^{2}-2 \alpha_{y} k-2 k_{Z}^{2} \cos \theta-\epsilon
\end{array}\right)\left(\begin{array}{c}
A_{k}^{(s)} \\
B_{k}^{(s)}
\end{array}\right)=0
$$

with $\epsilon=2 m E / \hbar^{2}, \alpha_{y}=e E_{y} /\left(4 m c^{2}\right), \alpha_{z}=e E_{z} /\left(4 m c^{2}\right)$, and $k_{Z}^{2}=-g e m B /\left(4 \hbar m_{0} c\right)$. Note that due to the negative electron charge $\alpha_{y}, \alpha_{z}<0$ for $E_{y}, E_{z}>0$ in our notation. One obtains the eigenenergy (divided by $\hbar^{2} / 2 m$ )

$$
\epsilon^{(s)}(k)=k^{2}+2 s \operatorname{sgn}\left(k-k_{0}\right) \sqrt{C(k)},
$$

with $C(k)=\left(\alpha_{y}^{2}+\alpha_{z}^{2}\right) k^{2}+2 k_{Z}^{2} k\left(\alpha_{y} \cos \theta-\alpha_{z} \sin \theta \sin \varphi\right)+k_{Z}^{4}$ and $k_{0}=-k_{Z}^{2}\left(\alpha_{y} \cos \theta-\alpha_{z} \sin \theta \sin \varphi\right) /\left(\alpha_{y}^{2}+\alpha_{z}^{2}\right)$ being the wave number at which the "energy gap" $E_{G}=4 \sqrt{C(k)}$ becomes smallest [see Fig. 2.1]. At a given energy $\epsilon$ outside the gap, we obtain a fourfold degeneracy, i.e. four allowed states with different $k$, but the same $\epsilon$. Note that it is not an energy gap in the strong sense, but more a "pseudo gap", since there are still two allowed states (one left-moving and one right-moving state) for energies in this region. However, we simply call it the energy gap in the following. The corresponding eigenfunctions are

$$
\phi_{k}^{(s)}(x)=\frac{1}{\sqrt{2 \pi} \sqrt{1+\left|a_{k}^{(s)}\right|^{2}}} e^{i k x}\left(\begin{array}{c}
a_{k}^{(s)} \\
1
\end{array}\right)
$$

with

$$
a_{k}^{(s)}=\frac{-i \alpha_{z} k-k_{Z}^{2} e^{-i \varphi} \sin \theta}{\alpha_{y} k+k_{Z}^{2} \cos \theta-s \operatorname{sgn}\left(k-k_{0}\right) \sqrt{C(k)}}
$$

and the spin expectation values are given by

$$
\left\langle\sigma_{x}+i \sigma_{y}\right\rangle_{k, s}=2 \frac{\left(a_{k}^{(s)}\right)^{*}}{1+\left|a_{k}^{(s)}\right|^{2}}, \quad\left\langle\sigma_{z}\right\rangle_{k, s}=\frac{-1+\left|a_{k}^{(s)}\right|^{2}}{1+\left|a_{k}^{(s)}\right|^{2}} .
$$




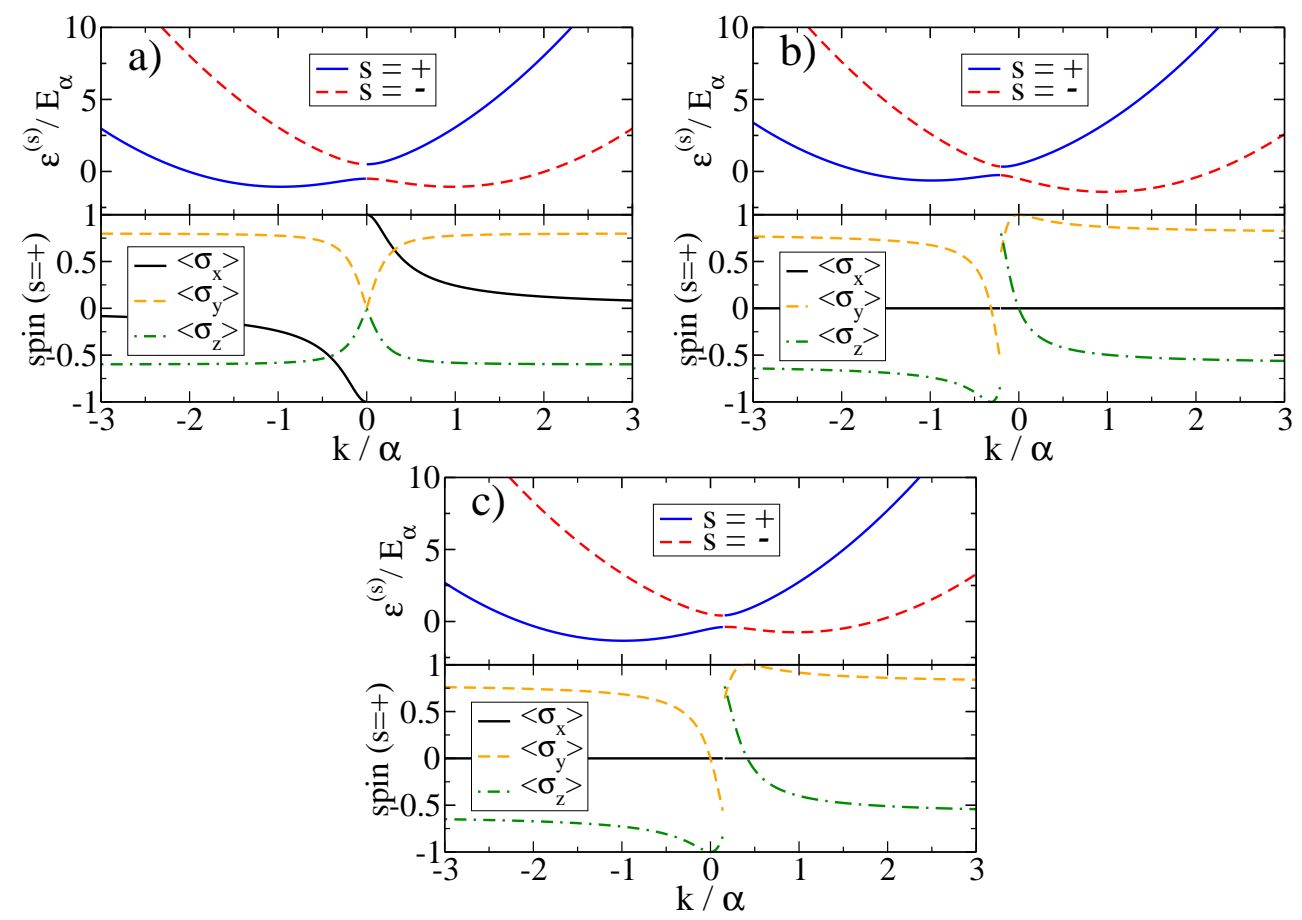

Figure 2.1: Dispersion and spin expectation values on the $(s=+)$-branch for a magnetic field in a) $x$ - , b) $y$ - and c) $z$-direction, $\alpha_{y} / \alpha=-0.6, \alpha_{z} / \alpha=-0.8$, $k_{Z} / \alpha=0.5$. The spin on the $(s=-)$-branch points in the opposite direction, i.e. $\left\langle\sigma_{i}\right\rangle_{k, s}=-\left\langle\sigma_{i}\right\rangle_{k,-s}$. The shape of the dispersion and the $k$-value at which the energy gap becomes smallest clearly depends on the direction of the magnetic field.

As can be seen from Eq. (2.24), the necessary condition $\left\langle\sigma_{x}\right\rangle_{k, s}^{2}+\left\langle\sigma_{y}\right\rangle_{k, s}^{2}+\left\langle\sigma_{z}\right\rangle_{k, s}^{2}=$ 1 holds for all values of $s$ and $k$. The existence of the confinement in $y$-direction (represented by $\alpha_{y}$ ) leads to a rotation of the spin out of the $x-y$-plain into the $z$ direction. This indicates that the ratio of $\alpha_{y}$ and $\alpha_{z}$ is crucial for the spin direction.

The energy dispersion Eq. (2.21) and the spin expectation values on the $(s=+)$ branch are shown in Fig. 2.1 as a function of $k$, with $k$ given in units of $\alpha=\sqrt{\alpha_{y}^{2}+\alpha_{z}^{2}}$ and the energy in units of $E_{\alpha}=\hbar^{2} \alpha^{2} / 2 m$. For $|k| \gtrsim \alpha$, the spin expectation values reach their asymptotic $k$-independent values. The spin on the $(s=-)$-branch points in the opposite direction, i.e. $\left\langle\sigma_{i}\right\rangle_{k, s}=-\left\langle\sigma_{i}\right\rangle_{k,-s}$, and is not shown explicitly here. In combination with the fact that for $\mathbf{B}=(B, 0,0)\left\langle\sigma_{y}\right\rangle_{k, s}$ and $\left\langle\sigma_{z}\right\rangle_{k, s}$ are symmetric with respect to $k=0$ on both branches, this explains why the ground 
state magnetization

$$
M_{i}=\int_{E_{0}}^{E_{F}} d \epsilon^{(s)}(k)\left\langle\sigma_{i}\right\rangle_{k, s} \quad, i=x, y, z
$$

vanishes in the $y$ - and $z$-direction for $\mathbf{B}$ being parallel to the wire. However, integrating $\left\langle\sigma_{x}\right\rangle_{k, s}$ according to Eq. (2.25) from the minimum $E_{0}$ of the energy dispersion up to the Fermi energy $E_{F}$ over all possible $k$-values and both $s$-branches accounting for Eq. (2.21) leads to a nonvanishing ground state magnetization in the $x$-direction, since $\left\langle\sigma_{x}\right\rangle_{k, s}=-\left\langle\sigma_{x}\right\rangle_{-k, s}$.

The energy gap is given by $4 \sqrt{C\left(k_{0}\right)}$ [see Eq. (2.21)] and does not necessarily decrease from its maximum value $4 k_{Z}^{2}$, if $\mathbf{B}$ is tilted against $\mathbf{e}_{x}$ as stated in Ref. [26]. In units of the Zeeman energy $E_{Z}=2 \hbar^{2} k_{Z}^{2} / 2 m$, the size of the gap $E_{G}$ for an arbitrary magnetic field $\mathbf{B}=B(\sin \theta \cos \phi, \sin \theta \sin \phi, \cos \theta)$ is given by

$$
\frac{E_{G}}{E_{Z}}=2-2 \frac{\left(\alpha_{y} \cos \theta-\alpha_{z} \sin \theta \sin \phi\right)^{2}}{\alpha^{2}} \text {. }
$$

Therefore, a finite $\alpha_{y}$ term is necessary for opening the gap for $\mathbf{B} \| \mathbf{e}_{y}$, i.e. $\theta=\phi=$ $\pi / 2$. We chose the parameter set $\left(\alpha_{y}, \alpha_{z}, k_{Z}\right) / \alpha=(-0.6,-0.8,0.5)$ in Fig. 2.1 in order to exemplify this effect.

In many experimental systems, the confining potential in the $y$-direction might be much weaker than in the $z$-direction. In this case $\left|\alpha_{y}\right| \ll\left|\alpha_{z}\right|$, but subband mixing becomes relevant. The latter strongly affects the spin-dependent transport properties as e.g. investigated in Ref. [29], and the polarization effects discussed here can be expected to vary or even disappear. A strong confinement in the $y$ direction leading to a sizable $\alpha_{y}$ is thus essential to achieve spin polarization in the present setup. The lower dispersion branch in Fig. 2.1 has a "W"-like shape. For $\mathbf{B}=(B, 0,0)$, the condition for this behavior is $\alpha_{y}^{2}+\alpha_{z}^{2}>2 k_{Z}^{2}$ and becomes much more complex for an arbitrary direction of the magnetic field. We now focus on the situation in which $\mathbf{B}=(B, 0,0)$ and investigate and compare the electronic transport and spin polarization at a potential step and a potential barrier.

\subsection{Transport and spin polarization}

At first, we implement a potential step in our model and calculate the transmission and spin polarization of an electron with a certain energy $E_{F}$ [see Fig. [2.2 a)]. Although such a potential step might be difficult to realize experimentally without any unintentional side-effects, it is nevertheless a very good model to get a first insight into spin-dependent transport.

This potential step setup was first suggested by Strěda and Sěba in Ref. [26]. However, they neglected $\alpha_{y}$ and therefore did not account for a sharp confinement in $y$-direction, which is neccessary to obtain a well-defined one-dimensional system. The transmissions $t_{s s^{\prime}}$ of an electron at fixed Fermi energy $E_{F}$ passing a potential 

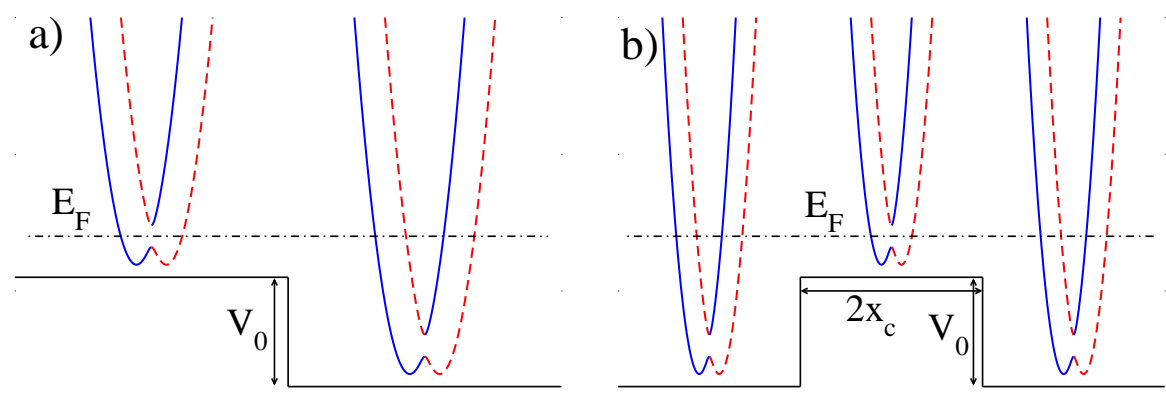

Figure 2.2: a) A potential step of height $V_{0}$ and b) a potential barrier of height $V_{0}$ and width $2 x_{c}$. The corresponding dispersions in the different regions are sketched (solid line: $s=+$, dashed line: $s=-$ ).

step in the wire direction [see Fig. 2.2 a)] are obtained by assuming continuity of the wave functions and their derivatives at the interface. It was argued in Ref. 36] that one has to consider the continuity of the wave function's flux and not simply its derivative, but in our setup both conditions lead to the same equations as we consider a homogeneous SOI. The first index in $t_{s s^{\prime}}$ labels the $s$-branch of the electron state on the left side and the second index labels the branch on the right side of the potential step.

Since we assume a translational invariant system, we can choose the $x$-coordinate such that the potential step is at $x=0$.

Therefore, the continuity condition of the wave function and its first derivative gives the system of linear equations

$$
\begin{aligned}
\phi_{k_{1}}^{(s)}(0)+A \phi_{k_{2}}^{(+)}(0)+B \phi_{k_{3}}^{(-)}(0) & =C \phi_{k_{4}}^{(+)}(0)+D \phi_{k_{5}}^{(-)}(0) \\
k_{1} \phi_{k_{1}}^{(s)}(0)+A k_{2} \phi_{k_{2}}^{(+)}(0)+B k_{3} \phi_{k_{3}}^{(-)}(0) & =C k_{4} \phi_{k_{4}}^{(+)}(0)+D k_{5} \phi_{k_{5}}^{(-)}(0)
\end{aligned}
$$

which needs to be solved for the coefficients $A, B, C, D$. $k_{1}$ is the wave number of the incoming wave with either $s=+$ or $s=-, k_{2}, k_{3}$ belong to the reflected wave with $s= \pm$ and $k_{4}, k_{5}$ belong to the transmitted wave with $s= \pm$, respectively. All wave numbers $k_{j}$ with $j \in\{1, \ldots, 5\}$ belong to the same Fermi energy $E_{F}$. For the right region of the potential step, i.e. for $k_{4}$ and $k_{5}$, one has to consider a dispersion shifted by $V_{0}$. Moreover, determining $k_{j}$ from the corresponding dispersion at given $E_{F}$, one has to account for the sign of the Fermi velocity $v_{j}$ at $k_{j}$ (left- or rightmoving states) to get a clear one-to-one correspondence between $k_{j}$ and $E_{F}$. The Fermi velocity is thereby given by the gradient of the dispersion at a certain $k$-value,

$$
v^{(s)}(k)=\frac{\partial \epsilon^{(s)}(k)}{\partial k} .
$$

One needs to be careful in the case in which the Fermi energy lies in the energy gap region, since there exists only one $s$-branch for propagating states, $s=-$ for rightmoving and $s=+$ for left-moving states. Instead of a plain wave $e^{i k x}$, an evanescent, 
i.e. exponentially decaying, wave $e^{-\tilde{k}|x|}$ must be used instead in Eq. (2.19) for the other $s$-value leading to the eigenfunction (for $\mathbf{B} \| \mathbf{e}_{x}$ )

$$
\psi_{\tilde{k}}(x)=\frac{1}{k_{Z} \sqrt{2\left(k_{Z}^{2}-\alpha_{z} \tilde{k}\right)}} e^{-\tilde{k}|x|}\left(\begin{array}{c}
i \alpha_{y} \tilde{k} \pm \sqrt{k_{Z}^{4}-\left(\alpha_{y}^{2}+\alpha_{z}^{2}\right) \tilde{k}^{2}} \\
-k_{Z}^{2}+\alpha_{z} \tilde{k}
\end{array}\right)
$$

which is normalized in spin space. One could multiply $\psi$ by $\sqrt{2 \tilde{k}}$ to get a normalization in the product of position and spin space. The sign in the first component in Eq. (2.29) depends on $\tilde{k}$, i.e. "+" for $\tilde{k}^{2}<k_{Z}^{4} /\left(\alpha_{y}^{2}+\alpha_{z}^{2}\right)$ and "-" for $\tilde{k}^{2}>k_{Z}^{4} /\left(\alpha_{y}^{2}+\alpha_{z}^{2}\right)$. The wave number $\tilde{k}$, belonging to a given $E_{F}$, can be obtained from the dispersion [see Eq. (2.21)] by allowing for an imaginary wave number $\kappa$ and setting $\tilde{k}=-i \kappa, \tilde{k} \in \mathbb{R}^{>0}$. Inserting $\psi$ instead of $\phi$ in Eq. (2.27) for the corresponding wave gives the continuity condition for the case with $E_{F}$ being within the energy gap.

The systems of linear equations for the different energy regions can be solved numerically or by computer algebra programs for the coefficients $A, B, C, D$ in Eq. (2.27). The transmission is just the absolute square of the corresponding coefficient multiplied by the ratio of the Fermi velocities, e.g. setting $s=-$ in Eq. (2.27) gives

$$
t_{-+}=|C|^{2} \frac{v_{4}}{v_{1}} \text { and } t_{--}=|D|^{2} \frac{v_{5}}{v_{1}} .
$$

$t_{++}$and $t_{+-}$are obtained analogously. The total transmission $T$ is the sum of the four components $t_{++}, t_{+-}, t_{-+}$, and $t_{--}$. To the right of the potential step and for momentum $|k| \gtrsim \alpha$, one can assign spins with quantum numbers $\uparrow, \downarrow$ and a properly chosen quantization axis to the branches $s=+,-$ because of the independence of $\langle\boldsymbol{\sigma}\rangle_{k, s}$ from $k$.

The polarization vector $\mathbf{P}=\left(P_{x}, P_{y}, P_{z}\right)$ is given by

$$
\mathbf{P}=\frac{t_{++}+t_{-+}}{T}\langle\boldsymbol{\sigma}\rangle_{k,+}+\frac{t_{+-}+t_{--}}{T}\langle\boldsymbol{\sigma}\rangle_{k,-} .
$$

\subsubsection{Homogenous system, $V_{0}=0$}

To get a first insight into the transport behavior of a system with SOI and magnetic field, we first of all consider the trivial limit $V_{0}=0$, i.e. a perfectly homogenous system. The four transmission components $t_{s s^{\prime}}$ with $s, s^{\prime}= \pm$ are shown in Fig. 2.3 a) as a function of the Fermi energy $E_{F}$ for $\alpha=2$. Choosing the energy offset such that $E_{F}=0$ corresponds to the middle of the energy gap leads to a minimum energy $\epsilon_{\min }$ for allowed states given by

$$
\frac{\epsilon_{\min }}{E_{Z}}=-\frac{\alpha^{4} / k_{Z}^{4}+1}{2 \alpha^{2} / k_{Z}^{2}} .
$$

The transmission $t_{++}$vanishes for energies inside the energy gap, but $t_{++}=1$ for $E_{F}$ being outside the gap, i.e. $E_{F} \in\left(\epsilon_{\min },-E_{Z}\right) \cup\left(E_{Z}, \infty\right)$, while $t_{--}=1$ and $t_{+-}=$ 

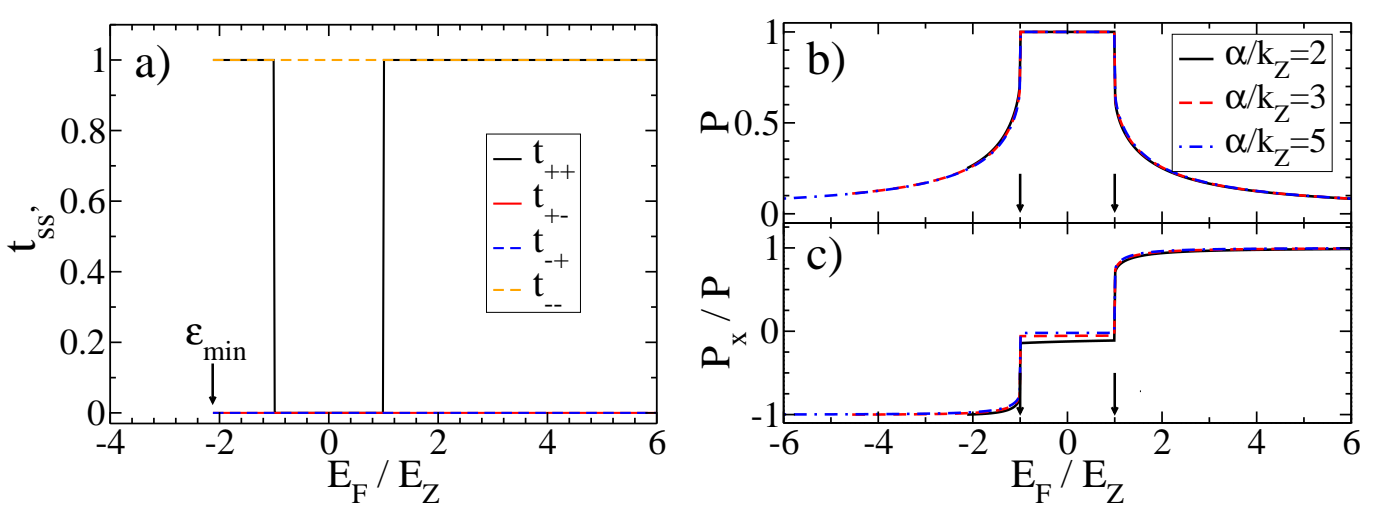

Figure 2.3: Transmission current and polarization for a homogeneous system, $V_{0}=0$, as a function of the Fermi energy $E_{F}$.

a) Transmission components $t_{s s^{\prime}}$ for $\alpha / k_{Z}=2$. The minimum energy $\epsilon_{\min }$ is marked by the arrow. $t_{++}=1(0)$ outside (inside) the energy gap reaching from $-E_{Z}$ to $E_{Z}$. $t_{--}=1$ and $t_{+-}=t_{-+}=0$ holds for all energies. Note that the components do not depend on $\alpha$.

b) Total polarization $P$ and c) contribution of $P_{x}$ for $\alpha / k_{Z}=2,3,5$. One observes $P=1$ inside the gap (indicated by the black arrows) and a drop outside the gap, almost independent of $\alpha . P_{x}$ is negligible inside the gap and decreases with increasing $\alpha$, but strongly dominates the total polarization outside the gap, independent of $\alpha$.

$t_{-+}=0$ holds for all energies. This behavior is independent of the SOI coupling parameters $\alpha_{y}$ and $\alpha_{z}$ and is also obtained in the trivial case $\alpha_{y}=\alpha_{z}=0$, where $\epsilon_{\min }$ corresponds to the lower boundary of the energy gap. The total polarization

$$
P=|\mathbf{P}|=\sqrt{P_{x}^{2}+P_{y}^{2}+P_{z}^{2}}
$$

and the polarization $P_{x}$ parallel to the wire (and the magnetic field) only depends on $\alpha$ and not on the separate choice of $\alpha_{y}$ and $\alpha_{z}$. The two SOI parameters only determine the ratio of the orthogonal polarization via $\left|\alpha_{y} / \alpha_{z}\right|=\left|P_{z} / P_{y}\right|$. As can be seen from Fig. 2.3 b) and c), the contribution of $P_{x}$ to the total polarization $P$ decreases with increasing $\alpha$, but $P$ itself is almost independent of $\alpha$.

The example of $V_{0}=0$ shows that spin polarization is possible without a potential step, which might seem astoninishing, since $\left\langle\sigma_{i}\right\rangle_{k, s}=-\left\langle\sigma_{i}\right\rangle_{k,-s}$. However, at given $E_{F}$ the allowed states on the $(s=+)$-branch have a different $k$-value than those on the $(s=-)$-branch, which leads to a finite spin polarization. But this spin polarization is homogenous in the entire system and one cannot control or modify it properly by adjusting the system parameters. 


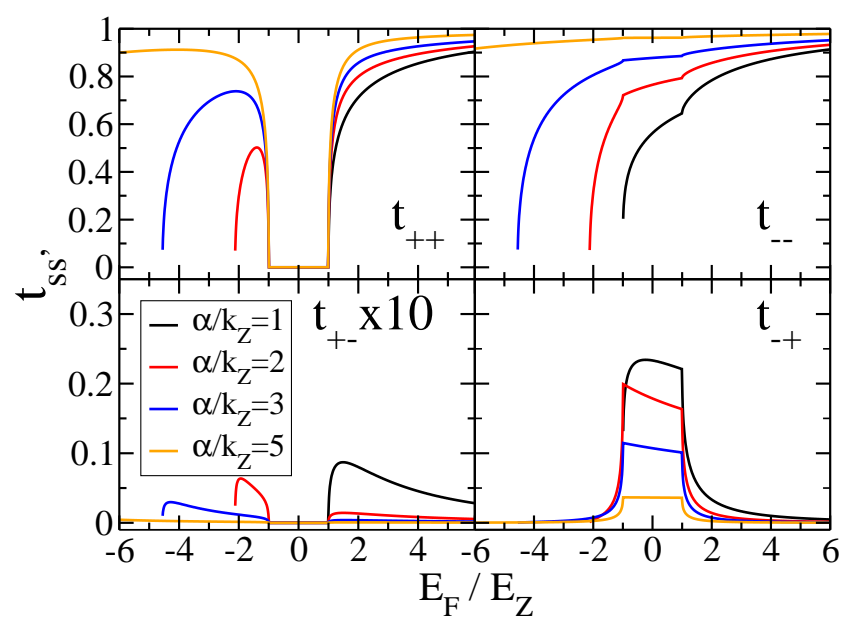

Figure 2.4: Transmission components at a potential step as a function of the Fermi energy for $V_{0} / E_{Z}=15$ and $\alpha / k_{Z}=1,2,3,5$. The $t_{++}$component is suppressed for energies in the gap, $E_{F} / E_{Z} \in(-1,1)$, where $t_{--}$dominates the total transmission and $t_{-+}$gets a growing impact. $t_{+-}$can be disregarded for all energies, whereas $t_{--}$becomes energy-independent for large $\alpha$ and tends towards unity.

\subsubsection{Potential step}

We now focus on a finite potential step $V_{0}>0$ and investigate, how spin polarized currents can be controlled by tuning the system parameters.

Fig. 2.4 shows the four transmission components $t_{s s^{\prime}}, s, s^{\prime}= \pm$, as a function of the Fermi energy for $V_{0} / E_{Z}=15$ and $\alpha / k_{Z}=1,2,3,5$. Referred to the Zeeman energy, the energy gap is given by $E_{F} / E_{Z} \in(-1,1)$. The components $t_{++}$and $t_{+-}$ are exactly zero in this region, since there are no propagating $(s=+)$-states in the left area of the potential step for these energies. Whereas $t_{+-}$can be disregarded for all energies, $t_{++}$has significant values outside the gap, which increase with increasing $\alpha . t_{--}$is strictly monotonic increasing with increasing energy and tends to the unitary limit for large $\alpha$, i.e. $t_{--} \rightarrow 1$, independent of the energy. $t_{-+}$ plays an important role for energies inside the gap and is drastically suppressed with increasing $\alpha$. It is clear from Figs. 2.1 and 2.2 that the Fermi energy needs to exceed a certain value $E_{0}$ in order to allow for propagating states and therefore a finite transmission. $E_{0}$ grows with decreasing $\alpha$ and is just the lower boundary of the energy gap for $\alpha / k_{Z}=1$.

As shown in Fig. 2.5 a), the total polarization $P=|\mathbf{P}|$ of the current passing the potential step is large for energies in the gap and increases with $\alpha$. Similar to the transmissions $t_{s s^{\prime}}, P$ as well as the parallel polarization $P_{x}$ depend only on $V_{0}, k_{Z}$, and $\alpha$ for $\mathbf{B} \| \mathbf{e}_{x}$ and not on $\alpha_{y}$ and $\alpha_{z}$ independently. The relevant energy scale of the polarization shown in Fig. 2.5 is given by $E_{Z}$, which defines the size of the gap [see Eq. (2.26)]. Therefore, energies are given in units of $E_{Z}$ 

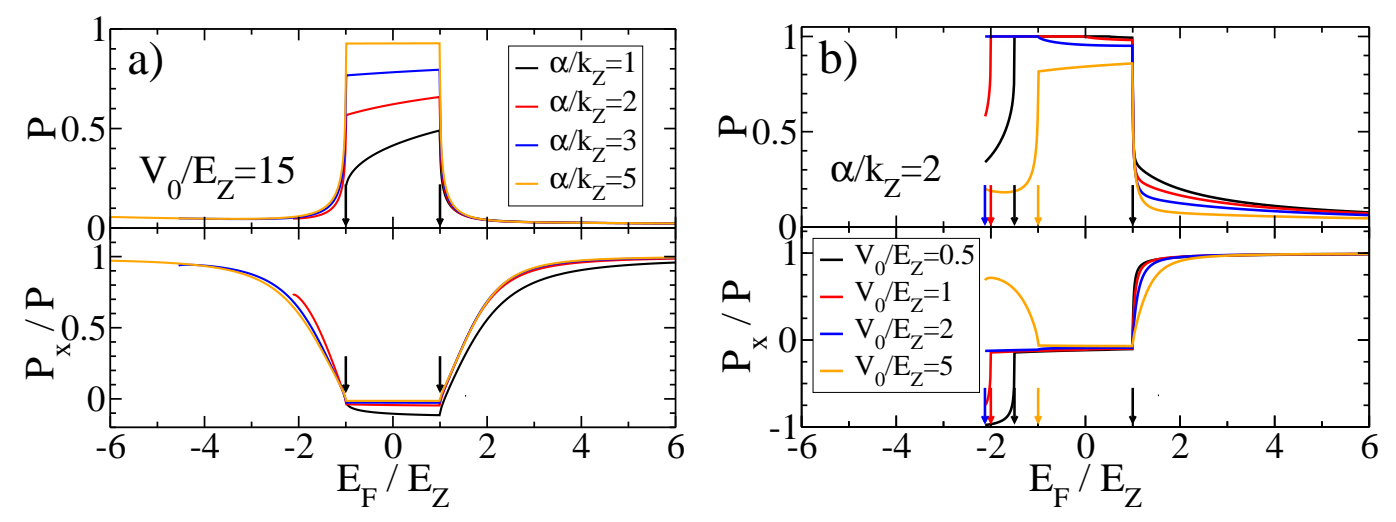

Figure 2.5: Polarization of the transmission current at a potential step as a function of the Fermi energy.

a) constant $V_{0} / E_{Z}=15$ and $\alpha / k_{Z}=1,2,3,5$. The total polarization $P$ is sizable for energies in the gap (indicated by the black arrows). In this regime it is mostly carried by $P_{y}$ and $P_{z}$. The polarization becomes negligible for energies outside the gap where $P_{x}$ dominates.

b) constant $\alpha / k_{Z}=2$ and $V_{0} / E_{Z}=0.5,1,2,5$. The arrows indicate the corresponding lower boundary of the energy gap (being either on the left or the right side of the step). The height of $V_{0}$ controls the sign of $P_{x}$ for small energies.

and wave vectors in units of $k_{Z}$. The parameters in Fig. 2.5 are $V_{0} / E_{Z}=15$, and $\alpha / k_{Z}=1,2,3,5$. The energy offset is chosen such that $E_{F} / E_{Z}=0$ corresponds to the middle of the gap. The parallel polarization $P_{x}$ gives the main contribution to the total polarization as the energy departs from the gap, $P_{x} / P \rightarrow 1$. For energies beyond the gap, however, the total polarization is negligible and within the gap, the parallel component plays an inferior role. The ratio of the two perpendicular polarizations is given by $\left|P_{z} / P_{y}\right|=\left|\alpha_{y} / \alpha_{z}\right|$. Therefore, the orthogonal polarization $\mathbf{P}_{\perp}=\left(0, P_{y}, P_{z}\right)$ can be rotated within the $y$ - $z$-plane by adjusting $\alpha_{y}$ and $\alpha_{z}$.

Fig. $2.5 \mathrm{~b}$ ) shows the total polarization and the relative polarization parallel to the wire (in $x$-direction) for constant SOI coupling $\alpha / k_{Z}=2$ and different height of the potential step, $V_{0} / E_{Z}=0.5,1,2,5$. The coloured arrows indicate the lower boundary of the energy gap of the dispersion on the right side of the potential step for the different $V_{0}$. For $V_{0} / E_{Z}<1$, there is an energy interval, which meets the energy gap on the left side of the step as well as the energy gap on the right side. For $E_{F} \in\left(\epsilon_{\min },-E_{Z}\right)$, both branches on the left side allow for propagating states, but there might be only one propagating (and one evanescent) state on the right side. Here, $\epsilon_{\min }$ denotes the minimum energy on the left side of the step.

Independent from whether or not there are one or two propagating states on the left side, i.e. whether or not $E_{F}$ is in or below the left energy gap, $P=1$, as long as $E_{F}$ meets the energy gap on the right side of the step.

Let us take the black curve in Fig. 2.5 b) $\left(V_{0} / E_{Z}=0.5\right)$ to clarify this. The 
left gap in units of $E_{Z}$ is given by the interval $(-1,1)$ and the minimum energy on the left side is $\epsilon_{\min } / E_{Z}=-17 / 8$ according to Eq. (2.32). Starting at $\epsilon_{\min }$, the polarization increases with energy until it reaches unity at $E_{F} / E_{Z}=-1.5$ which is the lower boundary of the right gap. At $E_{F} / E_{Z}=0.5$, the upper boundary of the right gap is reached and the polarization decreases slightly until the upper boundary of the left gap is reached at $E_{F} / E_{Z}=1$ and the polarization drops very sharply. The $x$-component $P_{x}$ of the polarization has the opposite sign at small energies compared to the regime in which $V_{0} \gg E_{Z}$. Moreover, Fig. 2.5 a) shows for the large $V_{0}$ regime that $P_{x}$ gains relative importance only for energies with almost negligible total polarization. However, in the regime $V_{0} \lesssim E_{Z}$, the total polarization is carried almost by $P_{x}$ alone for $E_{F}>E_{Z}$ and takes non-negligible values of up to 0.3 slightly above $E_{Z}$ [see Fig. [2.5 b)].

This very interesting case of small potential steps has not been reported in Refs. 26, 35, but might be of even greater importance for generating spin polarized currents than the case of large potential steps considerd in the literature so far, since in the small $V_{0}$ regime perfect spin polarization in the gap can be achieved for smaller SOI strength than in the large $V_{0}$ regime [see Fig. 2.5.

\subsubsection{Potential barrier}

We next study the transmission current at a potential barrier of height $V_{0}$ and width $2 x_{c}$ [see Fig. 2.2 b)]. This situation might be more realistic than a simple potential step, if one thinks of further structuring by applying gates to the quantum wire or inserting one or a small number of impurity atoms. The four transmission components $t_{s s^{\prime}}$ are obtained in exactly the same way as for the potential step. The only difference is that one has to take into account the continuity condition of the wave function and its first derivative at both ends $\left( \pm x_{c}\right)$ of the barrier.

\section{Large barrier height}

Fig. 2.6 a) shows the four components of the transmission as a function of $E_{F} / E_{Z}$ for $\alpha / k_{Z}=1,2,3, V_{0} / E_{Z}=15$, and $k_{Z} x_{c}=1$. Again, the SOI affects the transmissions $t_{s s^{\prime}}$ only via $\alpha$. The energy offset is chosen such that the energy gap in the middle region [see Fig. [2.2 b)] corresponds to the interval $\left(-E_{Z}, E_{Z}\right)$. Interestingly and in contrast to the potential step, the $s$-flipping transmissions are degenerate, $t_{+-}=t_{-+}$. This can be understood, if one considers the possible $s$ flips at the two interfaces leading to an overall $s$-flip. Labeling the left interface (1) and the right interface (2), one simply has to take the sum of the products of transmissions at each interface and obtains

$$
\begin{aligned}
& t_{+-}=t_{++}(1) t_{+-}(2)+t_{+-}(1) t_{--}(2) \\
& t_{-+}=t_{--}(1) t_{-+}(2)+t_{-+}(1) t_{++}(2)
\end{aligned}
$$

An analysis of the potential step problem shows that the $s$-conserving transmissions $t_{++}$and $t_{--}$are independent of the sign of $V_{0}$ and the $s$-flipping transmissions just 

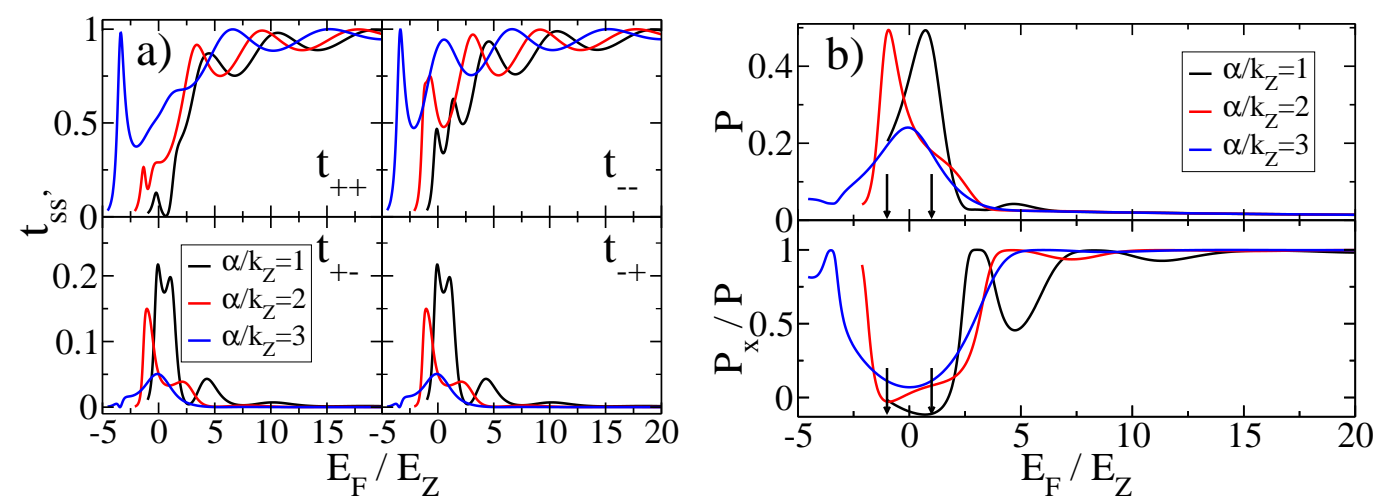

Figure 2.6: Partial transmissions and polarization at a potential barrier as a function of the Fermi energy $E_{F}$ for $\alpha / k_{Z}=1,2,3$. and barrier parameters $x_{c} k_{Z}=1$ and $V_{0} / E_{Z}=15$.

a) The high energy regime of $t_{++}$and $t_{--}$is dominated by strong oscillations. $t_{+-}$ and $t_{-+}$are degenerate and have their maximum values near the energy gap, but also have non-negligible values well beyond it.

b) The total polarization $P$ is sizable for energies well beyond the gap (indicated by the arrows) and shows oscillatory behavior. The $x$-component $P_{x}$ is only relevant in regimes in which the total polarization is small.

swap, i.e. $t_{+-}(1)=t_{-+}(2)$ and $t_{-+}(1)=t_{+-}(2)$. This leads to exactly the same values of $t_{+-}$and $t_{-+}$in Eq. (2.34). The exponential suppression of $t_{++}(1)$ and $t_{-+}(1)$ for energies within the gap does not affect this behavior. The $s$-conserving transmissions $t_{++}$and $t_{--}$show an oscillatory behavior, which is a well-known phenomenon of the standard quantum mechanical scattering problem at a potential barrier without SOI. However, especially for low energies, the amplitude strongly depends on $\alpha$. The $s$-flipping transmissions $t_{+-}$and $t_{-+}$oscillate as well. The second peak of $t_{++}$, which lies in the energy gap, is suppressed compared to $t_{--}$, since right-moving $(s=+)$-waves are exponentially damped in the barrier region and therefore, as shown in Ref. [26], $t_{--}$is the dominant component at each interface in this energy range.

Fig. 2.6 b) shows the total polarization $P$ and its $x$-component $P_{x} / P$ for the same parameters as in the left plot. Similarly to the potential step case, $P=|\mathbf{P}|$ and $P_{x}$ only depend on $\alpha$ and not on $\alpha_{y}$ and $\alpha_{z}$ independently. Surprisingly, the polarization now has a sizable value in an energy interval much bigger than the gap, which just reaches from $-E_{Z}$ to $E_{Z}$ [see the arrows in Fig. [2.6 b)]. This behavior must be contrasted to the polarization in the case of a potential step [see Fig. 2.5]. It can be traced back to the energy dependence of $t_{+-}$and $t_{-+}$shown in Fig. 2.6 a). Both have finite weight well beyond the energy gap. This might be due to interference effects of transmitted and reflected waves in the barrier region. 

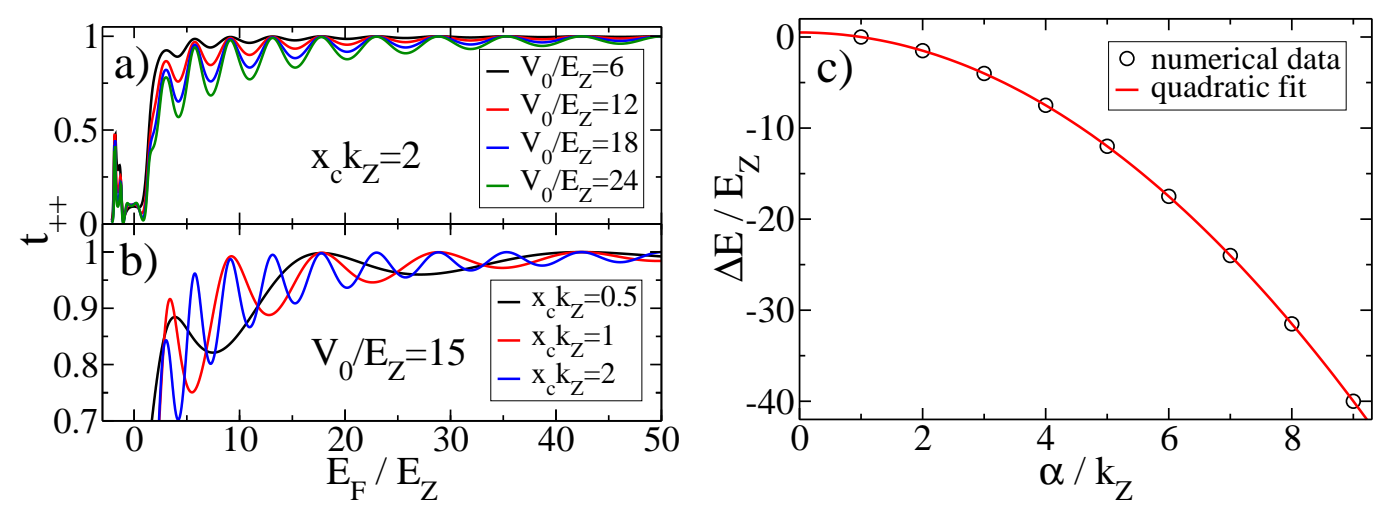

Figure 2.7: Transmission component $t_{++}$over the Fermi energy $E_{F}$ for $\alpha / k_{Z}=2$ and phase shift $\Delta E$ as a function of $\alpha$.

a) The barrier height $V_{0}$ only affects the oscillation amplitude but not its frequency for high energies.

b) The oscillation frequency is proportional to the barrier width $x_{c}$ for high energies.

c) The phase shift $\Delta E$ shows a perfectly quadratic dependence on $\alpha$.

\section{Transmission oscillations}

The oscillations of the transmission components $t_{++}$and $t_{--}$for high energies are another interesting feature which was not present in the simple potential step case. Figs. 2.7 a) and b) show the influence of the barrier parameters on the oscillatory behavior of the $t_{++}$component for constant SOI coupling $\alpha / k_{Z}=2$. Whereas the barrier height $V_{0}$ affects only the oscillation amplitude (for constant barrier width) and not the oscillation frequency, the barrier width $x_{c}$ leads to the opposite effect and one observes a clear proportional dependence of the oscillation frequency $\omega_{\text {osc }}$ on $x_{c}$, but the oscillations' envelope function is independent of $x_{c}$ for constant $V_{0}$. These results are in analogy to the spin-degenerate case (no SOI and no magnetic field) known from quantum mechanical textbooks. Moreover, the period of the oscillations is independent from $\alpha$ for high energies, but the oscillation phase is shifted by $\alpha$. Taking the $(\alpha=1)$-curve as reference, the shift $\Delta E$ of the curves is given by

$$
\frac{\Delta E}{E_{Z}}=0.5-0.5 \frac{\alpha^{2}}{k_{Z}^{2}}
$$

independent from the barrier parameters $V_{0}$ and $x_{c}$ [see Fig. $2.7 \mathrm{c}$ )]. All these results also hold for $t_{--}, t_{+-}$and $t-+$, but are not so obvious for the latter two due to the suppression of the $s$-flipping components for high energies.

\section{Small barrier height}

We now focus on the case in which $V_{0} / E_{Z} \lesssim 1$ is small and, therefore, one also has to consider the energy gaps of the dispersions on the left and right side of the 

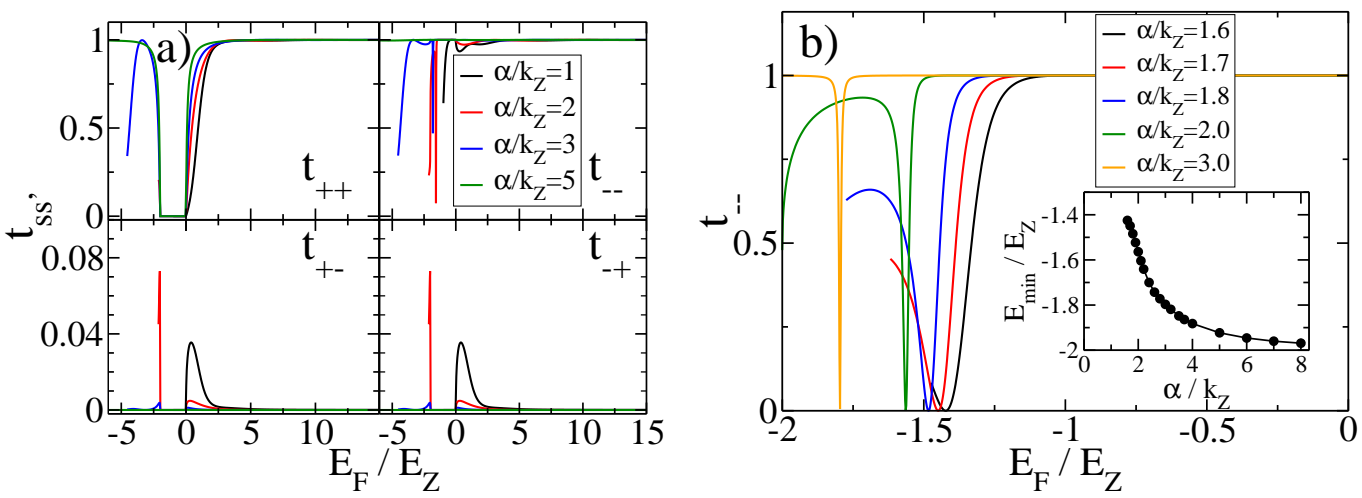

Figure 2.8: Transmission components $t_{s s^{\prime}}$ over the Fermi energy $E_{F}$ for $x_{c} k_{Z}=1$ and $V_{0} / E_{Z}=1$.

a) $t_{++}, t_{+-}$and $t_{-+}$vanish inside the energy gap $\left(-2 E_{Z}, 0\right)$ of the left/right side of the barrier, since there are no propagating states. $t_{--}$shows characteristic dips at certain energies depending on $\alpha$.

b) The transmission minima of $t_{--}$are shifted towards lower energies and their width decreases for increasing $\alpha$. The inset shows that the minima tend towards the lower boundary of the energy gap of the left/right side of the barrier.

barrier.

As can be seen from Fig. $2.8 \mathrm{a}$ ), the transmission components $t_{++}, t_{+-}$and $t_{-+}$ vanish in the energy gap of the left/right side of the barrier, which is the interval $\left(-2 E_{Z}, 0\right)$ for the barrier parameters $x_{c} k_{Z}=1$ and $V_{0} / E_{Z}=1$ chosen here. This is clear from the fact that states with this energy cannot propagate in the left/right region. The $s$-flipping components can be neglected, whereas $t_{++} \rightarrow 1$ outside the gap, except for the decay at the lower boundary $\epsilon_{\min }^{\mathrm{L}, \mathrm{R}}$ of the dispersion on the left/right side of the barrier given by

$$
\frac{\epsilon_{\min }^{\mathrm{L}, \mathrm{R}}}{E_{Z}}=-\frac{\alpha^{4} / k_{Z}^{4}+1}{2 \alpha^{2} / k_{Z}^{2}}-\frac{V_{0}}{E_{Z}} .
$$

The component $t_{--}$shows characteristic dips at certain energies which depend on $\alpha$. This is shwon in Fig. $2.8 \mathrm{~b}$ ). The inset shows the position $E_{\min }$ of the minimum of $t_{--}$as a function of $\alpha$. One observes a monotonic decay and a saturation

$$
\frac{E_{\min }}{E_{Z}} \rightarrow-\frac{V_{0}}{E_{Z}}-1 \quad \text { for } \alpha \rightarrow \infty
$$

which holds for arbitrary $V_{0} \lesssim E_{Z}$. Since there occur more dips for larger $x_{c}$, this might be a destructive interference effect resulting from reflected waves on both ends of the barrier.

It is interesting to note that the dips of $t_{--}$do not affect the spin polarization, neither for small nor for large barrier width $x_{c}$ [see Fig. 2.9]. Especially for small 

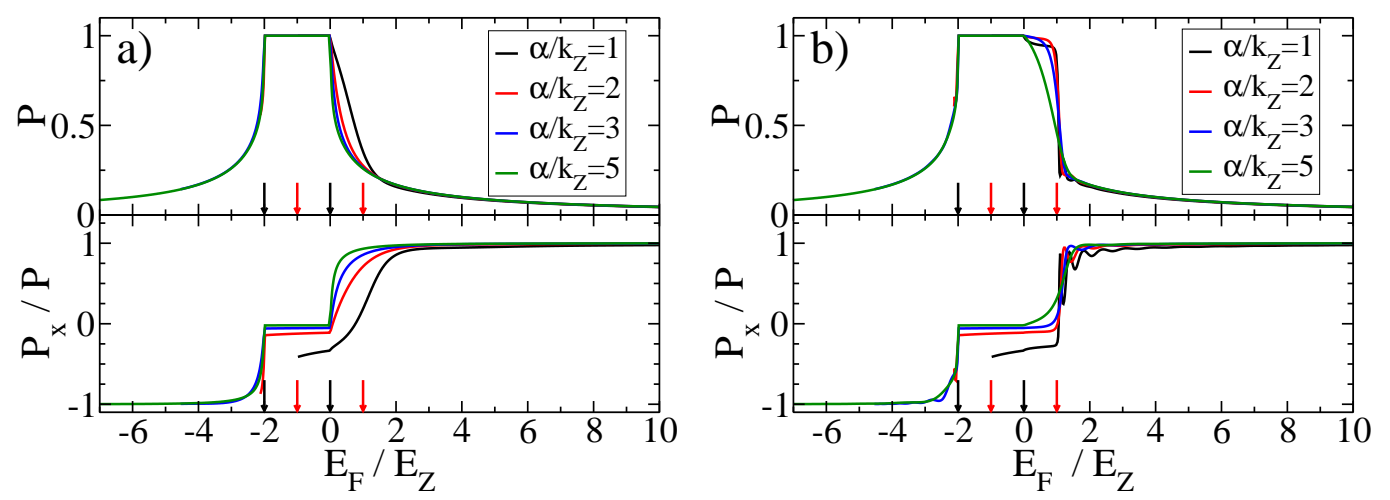

Figure 2.9: Total polarization and $x$-component $P_{x}$ (parallel to the wire) over the Fermi energy $E_{F}$ for small height of the potential barrier, $V_{0} / E_{Z}=1$. The black (red) arrows indicate the energy gap of the left/right (barrier) region.

a) small barrier width $\left(x_{c} k_{Z}=1\right)$ : the total polarization $P$ is comparable to the case $V_{0}=0$ [see Fig. 2.3] with the modification that the decay of $P$ at the upper boundary of the gap in the left/right region is slowed down for small $\alpha$. The parallel component $P_{x}$ shows the same qualitative behavior as in the $V_{0}=0$ case, but the increase of $P_{x}$ for energies between the upper boundary of the left/right gap and the upper boundary of the barrier gap is broadened for small $\alpha$.

b) large barrier width $\left(x_{c} k_{Z}=5\right)$ : the total polarization and $P_{x}$ strongly differs from the small $x_{c}$ regime for energies between the upper boundaries of the two energy gaps (between the right black and right red arrow), but coincides outside this interval. $P_{x}$ shows oscillations for energies slightly above the upper boundary of the energy gap in the barrier region. These oscillations become less pronounced with increasing $\alpha$.

$x_{c}$, a potential barrier with height $V_{0}=1$ does not seem to play a role for the polarization compared to the $V_{0}=0$ case shown in Fig. 2.3 Solely for small $\alpha$, one observes a difference for energies in the interval $E_{\mathrm{ub}}$ between the upper boundary of the energy gap on the left/right side of the barrier and the upper boundary of the energy gap in the barrier region (defined by the right black and right red arrow in Fig. (2.9). The decrease of $P$ is nearly linear in this interval for small $\alpha$, but shows the same steepness like in the $V_{0}=0$ case, if $\alpha$ becomes large. The same behavior is observed for the parallel component $P_{x}$, which also shows astonishing correspondence to the situation with vanishing barrier height except for energies in the interval $E_{\mathrm{ub}}$. Again, the tendency of reaching the asymptotic high energy polarization is slowed down for small $\alpha$. Fig. 2.9 b) shows the polarization for large barrier width $\left(x_{c} k_{Z}=5\right)$. The region in which $P \approx 1$ and $P_{x} \approx 0$, is enlarged by the interval $E_{\mathrm{ub}}$, if $\alpha$ becomes not too large. Moreover, $P_{x}$ shows oscillations for energies slightly above the upper boundary of the energy gap of the barrier region, which are stongly pronounced for small $\alpha$, but vanish as $\alpha$ is increased. 
The conditions for the experimental realization of a spin-polarized current are discussed in Ref. 26] and can be extended straightforwardly to the setups considered here. We will discuss this issue in detail in Chapter 7.

\subsection{Summary}

The starting point of this chapter was the expansion of the relativistic Dirac equation up to second order in $v / c$ yielding the SOI Hamiltonian. We implemented this Hamiltonian, represented by Rashba fields $\alpha_{y}$ and $\alpha_{z}$, into a homogenous continuum model for the one-dimensional motion (in $x$-direction) of non-interacting electrons. Furthermore, we accounted for a Zeeman effect induced by a magnetic field $\mathbf{B}$ in arbitrary direction and studied the eigenvalues (energy dispersion), eigenstates and spin expectation values of the resulting Hamiltonian. Besides the wave number $k$, an additional quantum number $s= \pm$ was introduced to unambigiously classify the different energy eigenvalues and eigenstates.

Being interested just in the low-energy behavior of the system, we focused on the lowest energy subband and omitted any subband mixing, which is an admissible simplification, since the energy subbands are well-separated in the spatially strongly confined electron systems under our consideration.

The quadratic dispersion of free electrons in 1D is spin-degenerate. We have shown that SOI leads to a splitting of the two degenerate parabulas in $k$-direction, whereas an additional magnetic field causes an energy gap due to Zeeman splitting. The interplay of SOI and Zeeman effect turned out to have also great influence on the spin expectation values allowing for almost every orientation of the spins by carefully tuning the parameters $\alpha_{y}, \alpha_{z}$ and $\mathbf{B}$.

In order to generate spin-polarized currents, which are of great experimental interest for spintronic applications, we then implemented a potential step in the system and studied the quantum mechanical scattering problem in terms of the transmissions $t_{s s^{\prime}}$ with $s, s^{\prime}= \pm$. The $t_{s s^{\prime}}$ as functions of the Fermi energy were shown to stongly depend on the SOI, especially in the magnetic field induced energy gap. For energies within the gap, we also observed significant spin polarization depending on the ratio of SOI and magnetic field and the height of the potential step. For large SOI fields, the polarization was shown to reach the limit of perfect polarization, $P \rightarrow 1$, within a certain energy range.

We also studied the experimentally more realistic situation of a potential barrier of finite width. Again, perfect polarization could be achieved by carefully tuning the system parameters. The oscillatory behavior of the transmission, which is wellknown for the scattering at a potential barrier in the absence of SOI, was also observed for the $t_{s s^{\prime}}$ and led to interesting interference phenomena.

In the next chapter, we will construct a lattice model with SOI and Zeeman term which shows the same low-energy behavior in terms of energy dispersion and spin expectation values. We will study the transport properties of the lattice model and compare them with the results obtained above for the continuum model. 


\section{Chapter 3}

\section{Transport properties of a non-interacting lattice model with SOI}

As discussed in the previous chapter in relation to the continuum model, spin-orbit interaction (SOI) can strongly alter the system's transport properties and generate spin-polarized currents. The polarization can be tuned by including a potential step or a potential barrier in the system.

However, it is a widely accepted fact that lattice models have given proof of being indispensible in mesoscopic solid state physics, not only due to many formal advantages [37, but also because they often model experimental situations in mesoscopic regimes far better than corresponding continuum models, especially in low dimensions.

Firstly, we aim at constructing an infinite tight-binding lattice model which shows, in appropriate parameter regimes, similar low-energy physics as the continuum model in terms of energy dispersion and spin expectation values. Secondly, we investigate how the transport properties regarding the linear conductance of this lattice model are modified by the SOI and whether spin polarization is possible for an appropriate potential structure.

In order to study transport phenomena, we will confine the lattice model under consideration to a finite size and attach it to two semi-infinite lattice systems without SOI and without a magnetic field. This situation is often found in experiments where a mesoscopic low-dimensional quantum system is attached to higher-dimensional leads.

\subsection{Lattice model with SOI and Zeeman term}

We start with a representation of the Hamiltonian in terms of Wannier states $|j, \sigma\rangle$ with $j \in \mathbb{Z}$ labeling the lattice site and $\sigma=\uparrow, \downarrow$ labeling the spin. The spin quantization is chosen along the $z$-direction. With $c_{j, \sigma}^{\dagger}$ being the creation operator of 
an electron at site $j$ with spin $\sigma$, the lattice model Hamiltonian for an arbitrary magnetic field $\mathbf{B}=B(\sin \theta \cos \varphi, \sin \theta \sin \varphi, \cos \theta)$ can be written as

$$
H=H_{0}+H_{\mathrm{pot}}+H_{R}+H_{Z},
$$

with the free part

$$
H_{0}=\epsilon \sum_{j, \sigma} c_{j, \sigma}^{\dagger} c_{j, \sigma}-t \sum_{j, \sigma}\left(c_{j+1, \sigma}^{\dagger} c_{j, \sigma}+c_{j, \sigma}^{\dagger} c_{j+1, \sigma}\right)
$$

containing the on-site energy and the conventional (spin-conserving) hopping, external potential (e.g. due to nano-device structuring)

$$
H_{\mathrm{pot}}=\sum_{j, \sigma} V_{j, \sigma} c_{j, \sigma}^{\dagger} c_{j, \sigma}
$$

the SOI (Rashba) hopping terms

$$
\begin{aligned}
H_{R}= & -\alpha_{z} \sum_{j, \sigma, \sigma^{\prime}}\left(c_{j+1, \sigma}^{\dagger}\left(i \sigma_{y}\right)_{\sigma, \sigma^{\prime}} c_{j, \sigma^{\prime}}+\text { H.c. }\right) \\
& +\alpha_{y} \sum_{j, \sigma, \sigma^{\prime}}\left(c_{j+1, \sigma}^{\dagger}\left(i \sigma_{z}\right)_{\sigma, \sigma^{\prime}} c_{j, \sigma^{\prime}}+\text { H.c. }\right),
\end{aligned}
$$

and the Zeeman term

$$
\begin{aligned}
H_{Z}= & \gamma B \sum_{j, \sigma, \sigma^{\prime}} c_{j, \sigma}^{\dagger}\left[\left(\sigma_{x}\right)_{\sigma, \sigma^{\prime}} \sin \theta \cos \varphi\right. \\
& \left.+\left(\sigma_{y}\right)_{\sigma, \sigma^{\prime}} \sin \theta \sin \varphi+\left(\sigma_{z}\right)_{\sigma, \sigma^{\prime}} \cos \theta\right] c_{j, \sigma^{\prime}} .
\end{aligned}
$$

The Zeeman coupling constant $\gamma=2 k_{Z}^{2} / B$ is introduced here in order to keep the notation short. The SOI Hamiltonian Eq. (3.5) consists of a spin-flip hopping $\alpha_{z}$ due to the confinement in $z$-direction which already was observed in Ref. [28] and, additionally, a complex spin-conserving hopping $\alpha_{y}$ due to confinement in $y$ direction. Note that if we would have chosen the spin quantization axis to point in $y$-direction, the $\alpha_{y}$-term would habe been modelled by a spin-flip hopping and the $\alpha_{z}$-term by a complex spin-conserving hopping. We assume a lattice constant $a=1$ in order to make the wave vector $k$ a dimensionless quantity (for convenience). All system parameters are assumed to be constant over the entire system, a condition we will drop later on. We show the analogy to the continuum case suppressing $H_{\text {pot }}$ and take as an ansatz for the corresponding eigenstates

$$
|k, s\rangle=\sum_{j, \sigma} a_{\sigma}^{s}(k) e^{i k j}|j, \sigma\rangle .
$$

This leads to the eigenenergies

$$
E^{(s)}(k)=\epsilon-2 t \cos k+2 s \operatorname{sgn}\left(k-k_{0}\right) \sqrt{D(k)},
$$


with

$$
k_{0}=\arcsin \left[-k_{Z}^{2}\left(\alpha_{y} \cos \theta-\alpha_{z} \sin \theta \sin \varphi\right) /\left(\alpha_{y}^{2}+\alpha_{z}^{2}\right)\right]
$$

and

$$
D(k)=\left(\alpha_{y}^{2}+\alpha_{z}^{2}\right) \sin ^{2} k+k_{Z}^{4}+2 k_{Z}^{2} \sin k\left(\alpha_{y} \cos \theta-\alpha_{z} \sin \theta \sin \varphi\right) .
$$

Eq. (3.7) has almost the same form as the continuum version Eq. (2.21). In fact, choosing the on-site energy $\epsilon=2 t$, which corresponds just to an overall energy shift, and substituting $\cos k$ by $1-k^{2} / 2$ and $\sin k$ by $k$, which is a valid approximation for sufficiently small $|k|$, we get exactly the same form. Note however that, in contrast to the continuum case, $\alpha_{y}, \alpha_{z}$ and $k_{Z}^{2}$ now have the unit of energy. We choose $a_{\downarrow}^{s}(k)=1$ for the eigenstates Eq. (3.6) and obtain $a_{\uparrow}^{s}(k)=c_{k}^{(s)}$ with

$$
c_{k}^{(s)}=\frac{-i \alpha_{z} \sin k-k_{Z}^{2} e^{-i \varphi} \sin \theta}{\alpha_{y} \sin k+k_{Z}^{2} \cos \theta-s \operatorname{sgn}\left(k-k_{0}\right) \sqrt{D(k)}}
$$

being dimensionless. Therefore, the lattice spin expectation values

$$
\left\langle\sigma_{x}+i \sigma_{y}\right\rangle_{k, s}=2 \frac{\left(c_{k}^{(s)}\right)^{*}}{1+\left|c_{k}^{(s)}\right|^{2}}, \quad\left\langle\sigma_{z}\right\rangle_{k, s}=\frac{-1+\left|c_{k}^{(s)}\right|^{2}}{1+\left|c_{k}^{(s)}\right|^{2}}
$$

have the same form as those in the continuum model, at least for small $|k|$. The energy dispersions and the spin expectation values for magnetic fields pointing in $x-, y$-, and $z$-direction are shown in Fig. 3.1. Besides the cosine-like structure, which becomes particularly relevant near the upper band edges $(k \approx \pm \pi)$, the dispersion and spin expectation values have the same shape as in the continuum model. A direct comparison of Fig. 3.1] and Fig. 2.1] shows that our lattice model reproduces the low energy physics, i.e. for $\left|k-k_{0}\right|<\pi / 2$, which have been observed in the continuum. Again, we only show the spin expectation values on the $(s=+)$-branch. The spin on the $(s=-)$-branch points in the opposite direction, i.e. $\left\langle\sigma_{i}\right\rangle_{k, s}=-\left\langle\sigma_{i}\right\rangle_{k,-s}$. The direct relation between the dispersion and the spin expectation values for energies of the order of the "gap" is the essential feature leading to the remarkable scattering properties of the continuum model (and eventually a spin polarized conductance) at steps and barriers. One can thus expect similar transport characteristics to be realized in the lattice model. In particular, one also obtains spin polarized currents in analogy to the continuum model by carefully tuning the system parameters, as will be shown below.

\subsection{Preliminary discussion about transport on a lattice}

Before we analyze the influence of the SOI on the transport properties of the lattice model, some preliminary discussion is neccessary. 


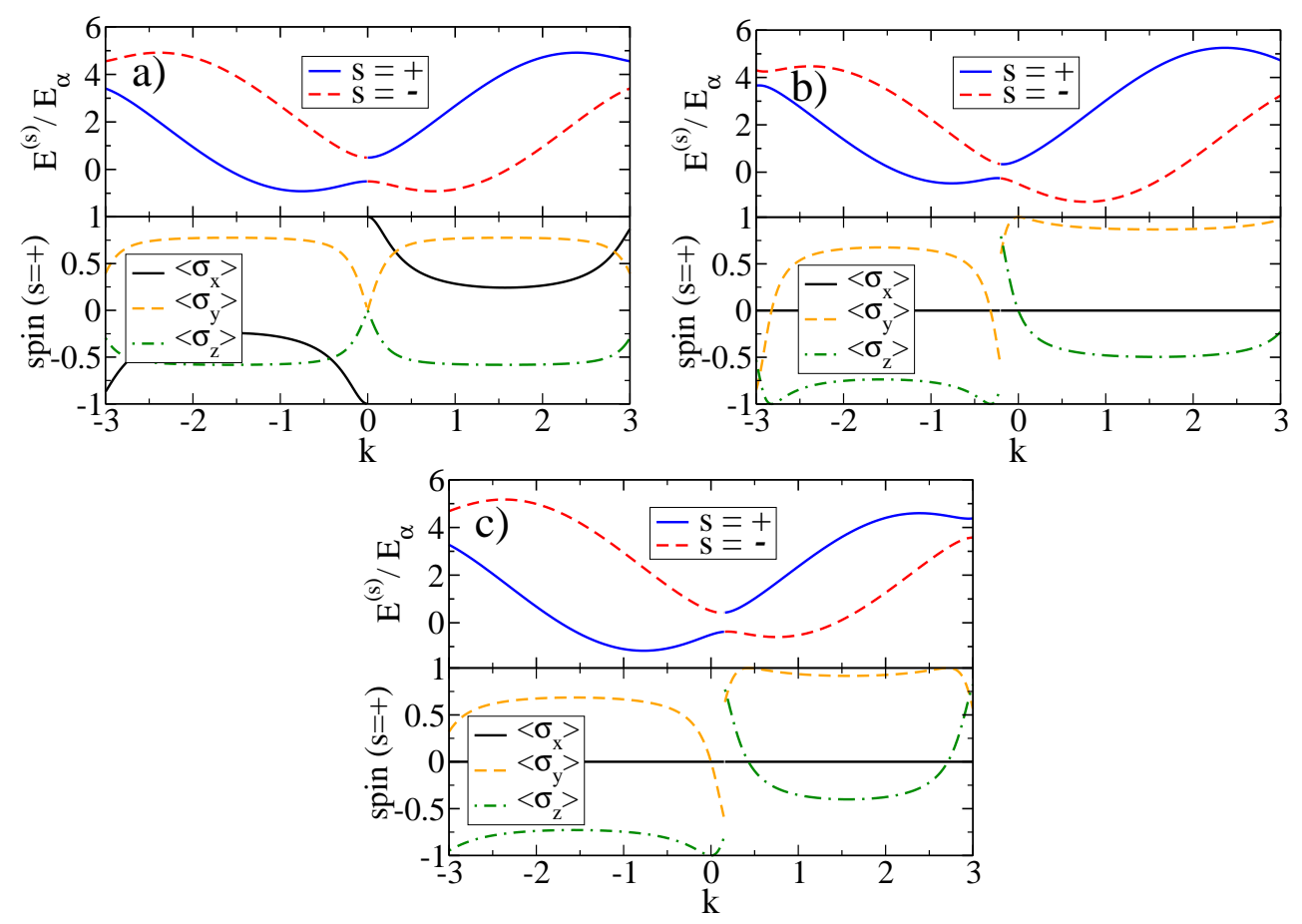

Figure 3.1: Lattice dispersion and spin expectation values on the $(s=+)$-branch for a magnetic field in a) $x$ - , b) $y$ - and c) $z$-direction for $t / \alpha=1, \alpha_{y} / \alpha=-0.6$, $\alpha_{z} / \alpha=-0.8, k_{Z} / \alpha=0.5$. The spin on the $(s=-)$-branch points in the opposite direction, i.e. $\left\langle\sigma_{i}\right\rangle_{k, s}=-\left\langle\sigma_{i}\right\rangle_{k,-s}$. For $\left|k-k_{0}\right|<\pi / 2$ one obtains exactly the same behavior as in the continuum case.

\subsubsection{Setup}

We couple a finite lattice system, consisting of $N$ lattice sites and being described by the Hamiltonian Eq. (3.1), to two semi-infinite leads which can be regarded as Fermi liquids (see Fig. 3.2). We will call the inner part the quantum wire or just the system for convenience.

The leads are modeled by semi-infinite lattices with conventional hopping $\tilde{t}_{L, R}$ and SOI (Rashba) hopping $\tilde{\alpha}_{y ; L, R}$ and $\tilde{\alpha}_{z ; L, R}$ with $L, R$ labeling the left/right lead. The lead Hamiltonian reads

$$
\begin{aligned}
H_{L, R}^{\text {lead }}=\sum_{j} \sum_{\sigma, \sigma^{\prime}} & {\left[d _ { j + 1 , \sigma } ^ { \dagger } \left(-\tilde{t}_{L, R} \delta_{\sigma, \sigma^{\prime}}+\tilde{\alpha}_{y ; L, R}\left(i \sigma_{z}\right)_{\sigma, \sigma^{\prime}}\right.\right.} \\
& \left.\left.-\tilde{\alpha}_{z ; L, R}\left(i \sigma_{y}\right)_{\sigma, \sigma^{\prime}}\right) d_{j, \sigma^{\prime}}+\text { H.c. }\right]
\end{aligned}
$$

with $d_{j, \sigma}^{\dagger}$ being the creation operator of an electron at lattice site $j$ with spin $\sigma$, whereupon $j=-\infty, \ldots, 0$ for the left lead and $j=N+1, \ldots, \infty$ for the right lead.

As will be seen below, we need to calculate the local components $g_{\sigma \sigma^{\prime}}(z)$ of the Green's function (local in the sense of a propagator along a closed path) in order 
to account for the leads' influence on the system properties. After a lengthy but straightforward calculation [see App. A] one obtains the local Green's function of the leads

$$
\begin{aligned}
& g_{\uparrow \uparrow}^{L, R}(z)=\frac{z+\mu_{L, R} \mp \sqrt{z^{2}+2 z \mu_{L, R}+\mu_{L, R}^{2}-4\left(\tilde{t}_{L, R}^{2}+\tilde{\alpha}_{y ; L, R}^{2}+\tilde{\alpha}_{z ; L, R}^{2}\right)}}{2\left(\tilde{t}_{L, R}^{2}+\tilde{\alpha}_{y ; L, R}^{2}+\tilde{\alpha}_{z ; L, R}^{2}\right)} \\
& g_{\uparrow \downarrow}^{L, R}(z)=0 \\
& g_{\downarrow \uparrow}^{L, R}(z)=0 \\
& g_{\downarrow \downarrow}^{L, R}(z)=\frac{z+\mu_{L, R} \mp \sqrt{z^{2}+2 z \mu_{L, R}+\mu_{L, R}^{2}-4\left(\tilde{t}_{L, R}^{2}+\tilde{\alpha}_{y ; L, R}^{2}+\tilde{\alpha}_{z ; L, R}^{2}\right)}}{2\left(\tilde{t}_{L, R}^{2}+\tilde{\alpha}_{y ; L, R}^{2}+\tilde{\alpha}_{z ; L, R}^{2}\right)} .
\end{aligned}
$$

The sign is chosen such that $\lim _{|z| \rightarrow \infty} g_{\sigma \sigma}(z)=0$. We have added chemical potentials $\mu_{L, R}$ for the left/right lead, since our analysis of the transport properties of the system will be performed within a grand-canonical calculation allowing for adding/removing electrons to the system. We consider the equilibrium situation $\mu_{L}=\mu_{R}$ in the following.

The fact that there are no spin-flip components in the leads' Green's function Eq. (3.13) can be understood by considering the different closed paths an electron can "choose" to propagate from the outermost lattice site of the lead into the system or further into the lead and back again. The reader will find a descriptive proof in App. A It is based on the time-inversion symmetry of the leads, which is not broken by SOI 38 .

The electrons can enter and leave the left and right lead via the hopping matrix elements $t_{L, R}$ (conventional hopping) and $\alpha_{y ; L, R}, \alpha_{z ; L, R}$ (Rashba hopping). The hybridization Hamiltonian describing the connection between the quantum wire and

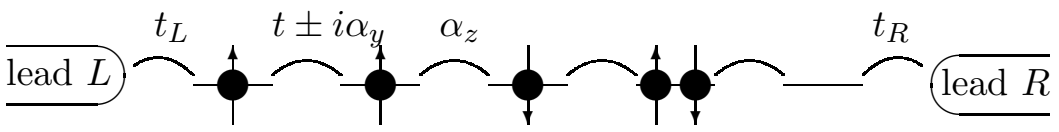

Figure 3.2: Linear geometry of the non-interacting quantum wire with conventional hopping $t$ and SOI parameters $\alpha_{y}$ and $\alpha_{z}$ connected to two semi-infinite non-interacting leads via hopping amplitudes $t_{L}$ and $t_{R}$. For convenience, the inner part is just called the system. 
the leads is given by

$$
\begin{aligned}
H_{\mathrm{QW}-\text { leads }}=\sum_{\sigma, \sigma^{\prime}} & {\left[c _ { 1 , \sigma } ^ { \dagger } \left(-t_{L} \delta_{\sigma, \sigma^{\prime}}+\alpha_{y ; L}\left(i \sigma_{z}\right)_{\sigma, \sigma^{\prime}}\right.\right.} \\
& \left.-\alpha_{z ; L}\left(i \sigma_{y}\right)_{\sigma, \sigma^{\prime}}\right) d_{0, \sigma^{\prime}} \\
& +d_{N+1, \sigma}^{\dagger}\left(-t_{R} \delta_{\sigma, \sigma^{\prime}}+\alpha_{y ; R}\left(i \sigma_{z}\right)_{\sigma, \sigma^{\prime}}\right. \\
& \left.\left.-\alpha_{z ; R}\left(i \sigma_{y}\right)_{\sigma, \sigma^{\prime}}\right) c_{N, \sigma^{\prime}}+\text { H.c. }\right] .
\end{aligned}
$$

In order to avoid backscattering into the left lead at the left interface and into the quantum wire at the right interface, all parameters of the quantum wire which are not present in the leads (i.e. magnetic field and SOI) are turned on/off adiabatically over a certain number $m_{1}$ of lattice sites with a smooth weight function

$$
\begin{aligned}
& f(j)=\frac{1}{2}\left(1-\cos \left(\frac{j}{m_{1}} \pi\right)\right) \quad \text { for } \quad j=1, \ldots, m_{1} \text { and } \\
& f(j)=\frac{1}{2}\left(1+\cos \left(\frac{j-1-N+m_{1}}{m_{1}} \pi\right)\right) \text { for } j=N-m_{1}, \ldots, N .
\end{aligned}
$$

We emphasize that the precise shape of this weight function does not have any influence on the results as long as $m_{1}$ is large enough and $N \gg 2 m_{1}$ [39].

We get rid of the leads by a Feshbach projection (see below), i.e. we project the influence of the left (right) lead to the leftmost (rightmost) lattice site of the quantum wire and end up with a $2 N \times 2 N$ problem for $N$ lattice sites of the quantum wire system (factor 2 is included because of the spin).

\subsubsection{Projection of the leads}

The reader can find a detailed description of the projection technique in e.g. Refs. [17, 40. We just resume the results here and show how this technique can be applied in one-to-one correspondence to the case with SOI, if one accounts for the $2 \times 2$-matrix algebra induced by the spin representation.

We define projection operators $P$ to the quantum wire and $Q$ to the leads, $P+Q=$ 1, and obtain for the Hamiltonian of the complete system

$$
\tilde{H}=\left(\begin{array}{cc}
\tilde{H}_{P P} & \tilde{H}_{P Q} \\
\tilde{H}_{Q P} & \tilde{H}_{Q Q}
\end{array}\right)
$$

As a result of this projection, the influence of the leads gives a contribution to the quantum wire's self energy $\Sigma_{i, j}\left(\sigma, \sigma^{\prime}\right)$. The left lead is projected onto the left boundary site $(j=1)$ of the wire and, similarly, the right lead is projected onto the right boundary site $(j=N)$ via

$$
\Sigma_{1,1}(z)=\left(\begin{array}{cc}
\left(t_{L}^{2}+\alpha_{y ; L}^{2}\right) g_{\uparrow \uparrow}^{L}(z)+\alpha_{z ; L}^{2} g_{\downarrow \downarrow}^{L}(z) & 0 \\
0 & \left(t_{L}^{2}+\alpha_{y ; L}^{2}\right) g_{\downarrow \downarrow}^{L}(z)+\alpha_{z ; L}^{2} g_{\uparrow \uparrow}^{L}(z)
\end{array}\right),
$$


$\Sigma_{N, N}(z)=\left(\begin{array}{cc}\left(t_{R}^{2}+\alpha_{y ; R}^{2}\right) g_{\uparrow \uparrow}^{R}(z)+\alpha_{z ; R}^{2} g_{\downarrow \downarrow}^{R}(z) & 0 \\ 0 & \left(t_{R}^{2}+\alpha_{y ; R}^{2}\right) g_{\downarrow \downarrow}^{R}(z)+\alpha_{z ; R}^{2} g_{\uparrow \uparrow}^{R}(z)\end{array}\right)$.

As can be seen from Eqs. (3.17) and (3.13) the leads' hopping contributions $\tilde{t}_{L, R}$, $\tilde{\alpha}_{y ; L, R}$ and $\tilde{\alpha}_{z ; L, R}$ enter the system's self energy via an effective hopping

$$
\tilde{t}_{L, R}^{\mathrm{eff}}=\sqrt{\tilde{t}_{L, R}^{2}+\tilde{\alpha}_{y ; L, R}^{2}+\tilde{\alpha}_{z ; L, R}^{2}} .
$$

Although it will be pointed out in App. A that a finite SOI in the leads might have consequences for the process of measuring spin polarization, we can disregard the Rashba hopping in the leads at this point and set $\tilde{t}_{L, R}=1$, i.e. all energies are given in units of the effective lead hopping. This choice of an energy scale will be used consequently throughout the remaining part of this thesis.

In addition to the fact that the lead contribution to the self energy of the system is diagonal in the spin index, because of $g_{\uparrow \downarrow}^{L, R}=g_{\downarrow \uparrow}^{L, R}=0$ [see Eq. (3.13)], we see from Eq. (3.17) that $\alpha_{z ; L, R}$ is the spin-flip hybridization and $t_{L, R}$ and $\alpha_{y ; L, R}$ couple to an effective spin-conserving hybridization. Therefore, we can abandon $\alpha_{y ; L, R}$ without loss of generality. If the spin-conserving components of the leads' Green's function are degenerate, $g_{\uparrow \uparrow}^{L}=g_{\downarrow \downarrow}^{L}$ and $g_{\uparrow \uparrow}^{R}=g_{\downarrow \downarrow}^{R}$, which is the case if no external magnetic field is applied to the leads, we have the same structure as in Eq. (3.18). Since we assume no magnetic field in the leads, we just take $t_{L, R}$ into account.

Note that the lead's contribution to the self energy can also be absorbed into the free propagator $\mathcal{G}_{0}$ of the wire, since the full propagator is given by the Dyson equation

$$
\mathcal{G}=\left(\mathcal{G}_{0}^{-1}-\Sigma\right)^{-1}
$$

which also holds in presence of Coulomb interaction [see Chapter 5 and 6 .

\subsubsection{The linear conductance}

As discussed in Ref. [41, the linear conductance $G$ is a convenient physical quantity for investigating transport properties of an electron system, since $G$ can be measured very accurately in voltage probe experiments even for more-terminal setups. On the other hand, the linear conductance can be calculated within the Landauer-Büttiker formalism. We want to resume the most important steps here.

The full conductance $G_{\text {total }}$ is normally defined as the proportionality factor between the current $I$ through a given sample and the voltage $V$ applied to it (Ohm's law) and is therefore a sample-specific quantity, in difference to the conductivity, which is just a property of the underlying material.

The linear conductance is given by the Kubo formula (see e.g. Ref. [42])

$$
G=\lim _{\omega \rightarrow 0} \Re\left(\frac{i e^{2}}{\omega} C_{I I}^{R}(\omega)\right)
$$


with $C_{I I}^{R}(\omega)$ being the Fourier transform of the time-dependent retarded currentcurrent correlation function

$$
C_{I I}^{R}\left(t-t^{\prime}\right)=-i \theta\left(t-t^{\prime}\right)\left\langle\left[\hat{I}(t), \hat{I}\left(t^{\prime}\right)\right]\right\rangle
$$

and $\hat{I}$ being the current operator.

According to the Landauer-Büttiker formalism (see e.g. Refs. [41] and [4]), the current $I$ is connected to the amplitude $\mathcal{T}$ of the transmission through the sample. Taking spin-flip terms into account, we have to assign the transmission amplitude with two spin indices $\mathcal{T} \rightarrow \mathcal{T}_{\sigma \sigma^{\prime}}$ with $\sigma, \sigma^{\prime} \in\{\uparrow, \downarrow\}$ with respect to a given spin quantization axis (chosen to point in the $z$-direction). Thus, the linear conductance (also assigned with two spin indices) at temperature $T$ and chemical potential $\mu$ can be written as

$$
G_{\sigma \sigma^{\prime}}(T, \mu)=\frac{e^{2}}{h} \int_{-W / 2-\mu}^{W / 2-\mu} d \epsilon\left(-\frac{\partial f}{\partial \epsilon}\right)\left|\mathcal{T}_{\sigma \sigma^{\prime}}(\epsilon)\right|^{2},
$$

with $W$ being the bandwith and $f(\epsilon)=1 /(\exp [\beta(\epsilon-\mu)]+1)$ being the Fermi function with $\beta=1 / T$ being the inverse temperature, setting the Boltzmann constant $k_{B}=1$.

In the following, we will always assume $T=0$. The derivative of the Fermi function with respect to $\epsilon$ therefore reduces to a (negative) $\delta$-function with the peak being at the chemical potential. The transmission probability can be expressed by the $(1, N)$ matrix element $\mathcal{G}(1, N)$ of the system's Green's function, i.e.

$$
\mathcal{T}_{\sigma \sigma^{\prime}}=2 t_{L} t_{R} \sin \left(k_{F}\right) \mathcal{G}_{\sigma \sigma^{\prime}}(1, N) .
$$

$k_{F}$ is the Fermi wave number defined by the leads energy dispersion

$$
\epsilon_{L, R}=-2 \tilde{t}_{L, R} \cos (k)=-2 \cos (k) \quad \text { with } \quad \tilde{t}_{L}=\tilde{t}_{R}=1 .
$$

As discussed above, we assume identical leads (left/right) and set $t_{L}=t_{R}=1$ (smooth contacts between the system and the leads). The chemical potential is the same all over the system and we obtain from Eq. (3.24)

$$
k_{F}=\arccos \left(-\frac{\mu}{2}\right) .
$$

We omit a detailed derivation of Eq. (3.23), but refer to Refs. 17, 40, in which the calculation has been carried out in great detail for the spinless case. For electrons with spin and in presence of SOI, the formulas remain the same with additionally assigning two spin indices to the transmission $\mathcal{T}_{\sigma \sigma^{\prime}}$ and the conductance $G_{\sigma \sigma^{\prime}}$ [see Eqs. [3.22) and [3.23)].

\subsection{The four components of the linear conductance}

We calculate the linear conductance as a function of the chemical potential $\mu$ which is the same in the leads and the quantum wire in the equilibrium situation considered 


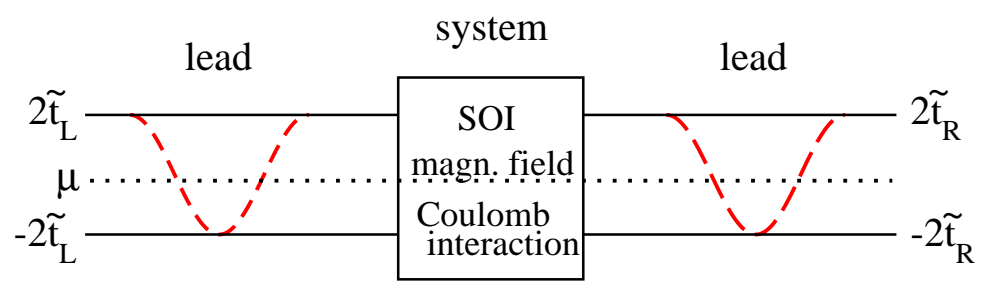

Figure 3.3: Setup for $\mu$-dependent calculation of the linear conductance. The dispersion of the non-interacting leads without SOI and magnetic field have a cosine-like shape (dashed curve). The chemical potential $\mu$ (dotted line) is tuned over the whole bandwidth of the leads. Since we choose $\tilde{t}_{L}=\tilde{t}_{R}$, the left lead and the right lead have the same width and dispersion. The influence of the Coulomb interaction on the transport properties of the system will be addressed in Chapter 5 and 6 .

here. We believe that this approach is more convenient than setting the filling of the entire system [17, 43, especially for experimental realization.

The setup is shown in Fig. 3.3. This corresponds to the energy-dependent calculations, which are common for continuum models [26, 35. The total conductance is given by the sum of the components $G_{\sigma \sigma^{\prime}}$ [defined in Eq. [3.22)]

$$
G_{\text {total }}=G_{\uparrow \uparrow}+G_{\uparrow \downarrow}+G_{\downarrow \uparrow}+G_{\downarrow \downarrow} .
$$

Due to the choice of the $z$-axis as spin quantization axis the spin polarization in $z$-direction can be defined as

$$
P_{z}=\frac{G_{\uparrow \uparrow}+G_{\downarrow \uparrow}-G_{\uparrow \downarrow}-G_{\downarrow \downarrow}}{G_{\text {total }}} .
$$

We emphasize that this definition differs from the continuum polarization which was defined in terms of spin expectation values [see Eq. (2.31)]. However, the lattice setup and consequently the process of measuring the polarization is somewhat different from the continuum version due to the presence of the leads. As we assume the SOI and the magnetic field to vanish in the leads, the spin is a conserved quantity there, i.e. the spin transport through the system is entirely determined by $G_{\sigma \sigma^{\prime}}$ and Eq. (3.27) is the correct definition of the spin polarization.

The conductance components and spin polarization in the $x$ - and $y$-direction can be obtained by a simple base transformation. The transmission amplitudes with respect to the $x$ - and $y$-direction as spin quantization axis are given by

$$
\begin{aligned}
& \mathcal{T}_{\uparrow \uparrow}^{(x)}=\left(\mathcal{T}_{\uparrow \uparrow}+\mathcal{T}_{\uparrow \downarrow}+\mathcal{T}_{\downarrow \uparrow}+\mathcal{T}_{\downarrow \downarrow}\right) / 2 \\
& \mathcal{T}_{\uparrow \downarrow}^{(x)}=\left(\mathcal{T}_{\uparrow \uparrow}-\mathcal{T}_{\uparrow \downarrow}+\mathcal{T}_{\downarrow \uparrow}-\mathcal{T}_{\downarrow \downarrow}\right) / 2 \\
& \mathcal{T}_{\downarrow \uparrow}^{(x)}=\left(\mathcal{T}_{\uparrow \uparrow}+\mathcal{T}_{\uparrow \downarrow}-\mathcal{T}_{\downarrow \uparrow}-\mathcal{T}_{\downarrow \downarrow}\right) / 2 \\
& \mathcal{T}_{\downarrow \downarrow}^{(x)}=\left(\mathcal{T}_{\uparrow \uparrow}-\mathcal{T}_{\uparrow \downarrow}-\mathcal{T}_{\downarrow \uparrow}+\mathcal{T}_{\downarrow \downarrow}\right) / 2
\end{aligned}
$$


and

$$
\begin{aligned}
& \mathcal{T}_{\uparrow \uparrow}^{(y)}=\left(\mathcal{T}_{\uparrow \uparrow}-i \mathcal{T}_{\uparrow \downarrow}+i \mathcal{T}_{\downarrow \uparrow}+\mathcal{T}_{\downarrow \downarrow}\right) / 2 \\
& \mathcal{T}_{\uparrow \downarrow}^{(y)}=\left(-i \mathcal{T}_{\uparrow \uparrow}+\mathcal{T}_{\uparrow \downarrow}+\mathcal{T}_{\downarrow \uparrow}+i \mathcal{T}_{\downarrow \downarrow}\right) / 2 \\
& \mathcal{T}_{\downarrow \uparrow}^{(y)}=\left(i \mathcal{T}_{\uparrow \uparrow}+\mathcal{T}_{\uparrow \downarrow}+\mathcal{T}_{\downarrow \uparrow}-i \mathcal{T}_{\downarrow \downarrow}\right) / 2 \\
& \mathcal{T}_{\downarrow \downarrow}^{(y)}=\left(\mathcal{T}_{\uparrow \uparrow}+i \mathcal{T}_{\uparrow \downarrow}-i \mathcal{T}_{\downarrow \uparrow}+\mathcal{T}_{\downarrow \downarrow}\right) / 2 .
\end{aligned}
$$

Inserting Eqs. (3.28) and (3.29) into Eq. (3.22) yields the conductance components $G_{\sigma \sigma^{\prime}}^{(x)}$ and $G_{\sigma \sigma^{\prime}}^{(y)}$. We then receive the spin polarization in $x$ - and $y$-direction according to Eq. (3.27). The total conductance is independent from the direction of the spin quantization axis. If not written down explicitely, $G_{\sigma \sigma^{\prime}}$ denotes the conductance components with respect to the $z$-direction.

Before we consider correlation effects [see Chapter 5 ] and [6], we firstt investigate the simpler situation without Coulomb interaction here and discuss the influence of the magnetic field and the SOI on the conductance components and the spin polarization. In the following, we choose the SOI parameters $\alpha_{y}, \alpha_{z}>0$ in contrast to Chapter 2 and Fig. 3.1. However, as we have shown in Chapter 2] the SOI parameters are given by $\alpha_{i}=e E_{i} /\left(4 m c^{2}\right), i=y, z$, with $e$ being the (negative) electron charge and $m$ being the effective mass. The electric field $E_{i}$ as the gradient of the confining scalar potential can, naturally, be negative here leading to $\alpha_{y}, \alpha_{z}>0$. However, we emphasize that the total conductance is independent of the sign of $\alpha_{y}$ and $\alpha_{z}$ and the polarization components $P_{i}$ might merely change their sign. We think that this approach with $\alpha_{y}, \alpha_{z}>0$ is more convenient, as all other hopping amplitudes are also positive numbers. This convention is also quite intuitive, if one thinks of the hopping amplitudes being expectation values of the kinetic energy related to some electron hopping processes.

Fig. 3.4 shows the conductance of a system with $N=100$ lattice sites and hopping $t=1$ as a function of the chemical potential $\mu$ in presence of SOI $\left(\alpha_{y}=0.3, \alpha_{z}=\right.$ $0.5)$. The system is perfectly coupled to the leads, i.e. $t_{L}=t_{R}=1$. As can be seen from Fig. 3.4 a), a sharply turned on/off SOI $\left(m_{1}=0\right)$ leads to oscillations of the total conductance even in the energy range far away from the band edges, since the SOI can be seen as hopping impurities in this case. Furthermore, one observes a drastic reduction of the conductance at the band edges. Obviously, it is sufficient to turn on/off the SOI smoothely over $m_{1}=20$ to avoid these oscillations and obtain a perfect conductance $G_{\text {total }}=2 e^{2} / h$ over the entire band width of the leads, $\mu \in(-2, \ldots, 2)$ [see Fig. $3.4 \mathrm{~b})$ ].

Although the conductance components strongly depend on the ratio of $t, \alpha_{y}$ and $\alpha_{z}$, the total conductance depends on these hopping terms only via

$$
t_{\mathrm{eff}}=\sqrt{t^{2}+\alpha_{y}^{2}+\alpha_{z}^{2}}
$$




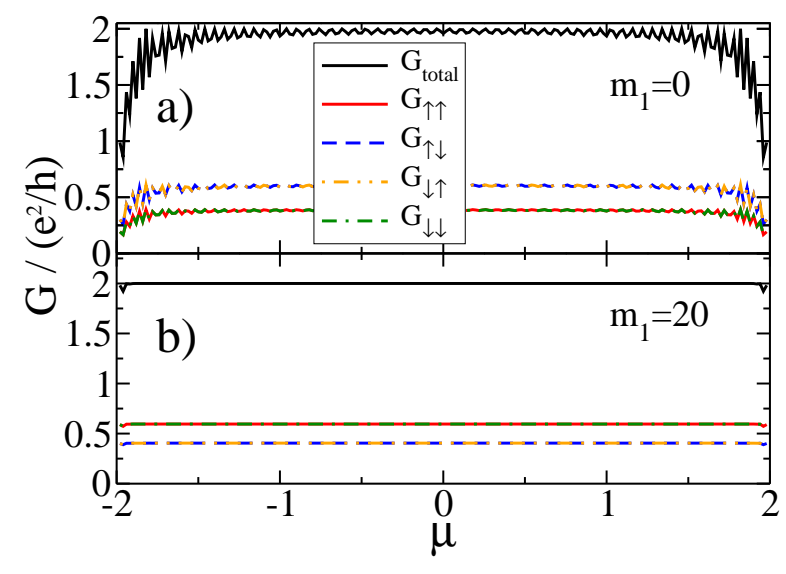

Figure 3.4: Total conductance and the four conductance components over the chemical potential $\mu$ for a system parameters $N=100, t=t_{L}=t_{R}=1, \alpha_{y}=0.3$, $\alpha_{z}=0.5$.

a) Turning on/off the SOI $\left(\alpha_{y}, \alpha_{z}\right)$ sharply leads to oscillations and a severe reduction of the conductance near the band edges.

b) The SOI is turned on/off smoothly over $m_{1}=20$ lattice sites. This ensures perfect conductance over the whole band width and a suppression of the oscillations.
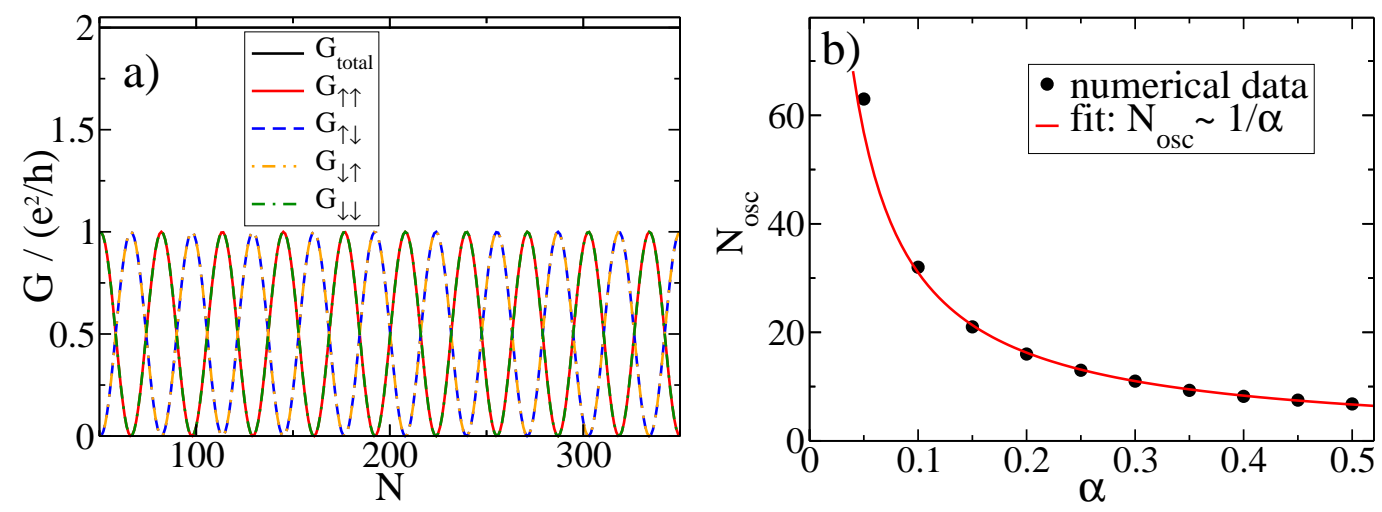

Figure 3.5: a) Total conductance and the four conductance components over the number of lattice sites in presence of SOI. The system is perfectly coupled to the leads $\left(t_{L}=t_{R}=1\right)$ and the SOI $\left(\alpha_{y}=0, \alpha_{z}=0.1\right)$ is turned on/off smoothly over $m_{1}=20$ lattice sites to obtain a perfect total conductance. The components are pairwise degenerate, $G_{\uparrow \uparrow}=G_{\downarrow \downarrow}, G_{\uparrow \downarrow}=G_{\downarrow \uparrow}$, and show a characteristic oscillatory behavior.

b) Oscillation period for different SOI strengths. The numerical data can be fitted well by a hyperbola, $N_{\text {osc }} \sim 1 / \alpha$. 
Due to rotational symmetry around the $x$-axis, $G_{\sigma, \sigma^{\prime}}^{(x)}$ depends only on the total SOI strength $\alpha=\sqrt{\alpha_{y}^{2}+\alpha_{z}^{2}}$. Since no external potential or magnetic field is present, the two spin-conserving and the two spin-flipping components are degenerate for every direction, i.e. $G_{\uparrow \uparrow}^{(x, y, z)}=G_{\downarrow \downarrow}^{(x, y, z)}$ and $G_{\uparrow \downarrow}^{(x, y, z)}=G_{\downarrow \uparrow}^{(x, y, z)}$. Clearly, there are no spin-flipping components in $y$ - $(z-)$ direction, if $\alpha_{y}=0\left(\alpha_{z}=0\right)$.

It has been pointed out in Ref. 3] that the ratio of spin-conserving and spinflipping conductance components depends on the system length. Plotting the conductance components as a function of the system length $N$ for constant $\mu$, one finds an oscillatory behavior with a period $N_{\text {osc }}$ depending on the strength of the Rashba hopping [see Fig. 3.5] a)]. As can be seen from Fig. 3.5 b), the period is inversely proportional to $\alpha$

$$
N_{\mathrm{osc}} \sim \frac{1}{\alpha}
$$

at least if $\alpha$ does not become too large.

\subsection{Interplay of SOI and magnetic field}

In addition to the SOI, we now account for a finite magnetic field $B_{x}$ (in direction of the wire) which is also turned on/off smoothly over $m_{1}=20$ lattice sites in order to avoid backscattering and oscillations of the total conductance.

Fig. 3.6] shows the effect of a magnetic field $\gamma B_{x}=0.6$ on the conductance of a system with $N=100$ lattice sites. The energy range with two conducting channels, i.e. $G_{\text {total }}=2 e^{2} / h$, is shrinked from both sides by $\gamma B_{x}$, which is clear if one considers the Zeeman effect on the components $G_{\sigma, \sigma^{\prime}}^{(x)}$. Besides the fact that the spin-flip components vanish, $G_{\uparrow \downarrow}^{(x)}=G_{\downarrow \uparrow}^{(x)}$, there are no propagating states in the spin-up subband for $\mu \in\left(-2,-2+\gamma B_{x}\right)$, and, analogously, there are no propagating states in the spin-down subband for $\mu \in\left(2-\gamma B_{x}, 2\right)$. However, it is much more interesting to plot the components $G_{\sigma, \sigma^{\prime}}^{(z)}$, because they show an oscillatory behavior which is not clear a priori due to base rotation of the $G_{\sigma, \sigma^{\prime}}^{(x)}$. Since the magnetic field is orthogonal to the spin quantization axis, the degeneracy $G_{\uparrow \uparrow}=G_{\downarrow \downarrow}, G_{\uparrow \downarrow}=G_{\downarrow \uparrow}$ still holds.

This degeneracy is lifted, if one considers a finite SOI. As can be seen from Fig. (3.7), the behavior of the conductance components strongly depends on the angle $\phi=-\arctan \left(\alpha_{y} / \alpha_{z}\right)$ between the spin quantization axis and the effective SOI field which is defined by

$$
\vec{\alpha}=\left(0, \alpha_{y}, \alpha_{z}\right)=\alpha(0,-\sin \phi, \cos \phi) .
$$

For $\phi \in\{0, \pi\}, \alpha_{y}=0$ and $\alpha_{z}=0.8$, we observe a degeneracy $G_{\uparrow \uparrow}=G_{\downarrow \downarrow}$ over the entire band, whereas the degeneracy $G_{\uparrow \downarrow}=G_{\downarrow \uparrow}$ only holds in the middle region and is lifted slightly outside this region. Still, there are oscillations of the conductance components as a function of $\mu$ in the middle region, but the energy period seems to be enlarged by the SOI compared to Fig. 3.6. 


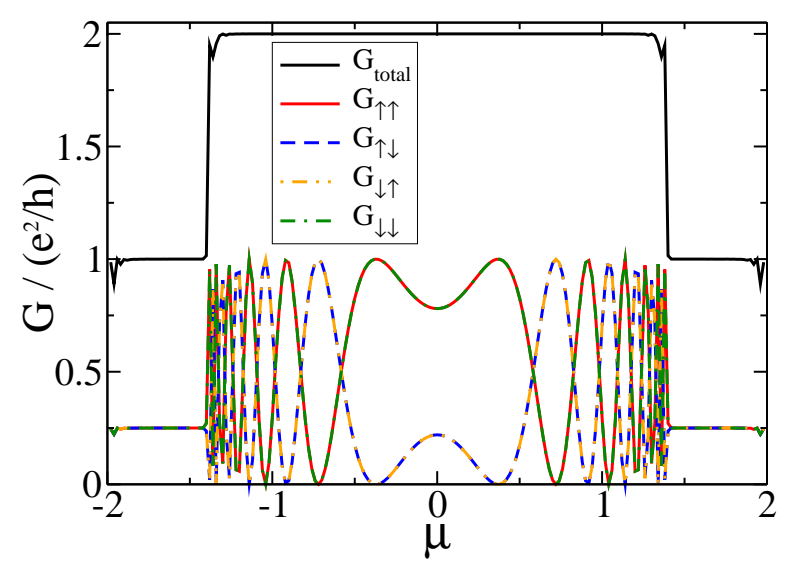

Figure 3.6: Conductance components over the chemical potential in presence of a magnetic field without SOI. The system parameters are $N=100, m_{1}=20, t=1$, $t_{L}=t_{R}=1, \gamma B_{x}=0.6$. The total conductance has plateaus at $G_{\text {total }}=1 e^{2} / h$ of width $\gamma B_{x}$ at the band edges. In this area there is only one conducting channel due to the Zeeman splitting. The four components reveal a fourfold degeneracy in the area with one conducting channel and a severe spin precession in the area with two conducting channels while being pairwise degenerate, $G_{\uparrow \uparrow}=G_{\downarrow \downarrow}, G_{\uparrow \downarrow}=G_{\downarrow \uparrow}$.

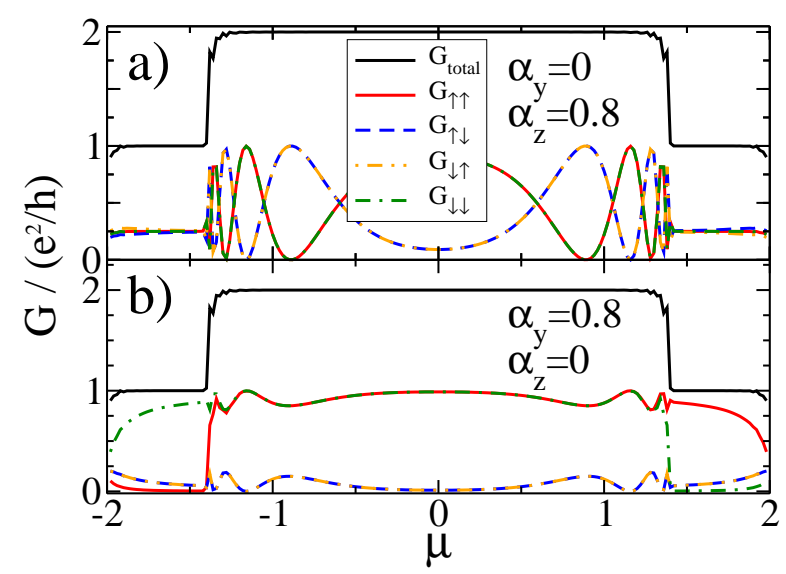

Figure 3.7: Conductance components over the chemical potential in presence of a magnetic field and SOI. The system parameters are $N=100, m_{1}=20, t=1$, $t_{L}=t_{R}=1, \gamma B_{x}=0.6$.

a) For $\alpha_{y}=0$ and $\alpha_{z}=0.8$, there is a degeneracy $G_{\uparrow \uparrow}=G_{\downarrow \downarrow}$ over the entire band, whereas the degeneracy $G_{\uparrow \downarrow}=G_{\downarrow \uparrow}$ only holds in the middle region and is lifted slightly outside this region.

b) For $\alpha_{y}=0.8$ and $\alpha_{z}=0$, we obtain $G_{\uparrow \downarrow}=G_{\downarrow \uparrow}$ for all $\mu$. There are only small oscillations, but a very large split of $G_{\uparrow \uparrow}$ and $G_{\downarrow \downarrow}$ outside the middle region. 
For $\phi=\pi / 2\left(\alpha_{y}=0.8, \alpha_{z}=0\right), G_{\uparrow \downarrow}=G_{\downarrow \uparrow}$ holds for all $\mu$. The oscillations of the components almost vanish, but there is a very large split of $G_{\uparrow \uparrow}$ and $G_{\downarrow \downarrow}$ outside the middle region. Note however that

$$
\begin{aligned}
& G_{\uparrow \uparrow}(\mu)=G_{\downarrow \downarrow}(-\mu) \\
& G_{\uparrow \downarrow}(\mu)=G_{\downarrow \uparrow}(-\mu),
\end{aligned}
$$

independent of $\phi$.

\subsubsection{Potential step}

We now implement a potential step into our setup. The potential of height $V_{0}$ is turned on smoothly with the same function as $\alpha_{y}, \alpha_{z}$ and $B_{x}$ and is turned off sharply in the middle of the system as shown in Fig. 3.8. The three different regions (left lead, quantum wire, right lead) are plotted with their corresponding energy dispersion. The leads have a cosine-like dispersion, whereas there are two nondegenerate branches (distinguished by some quantum number $s= \pm$ ) in the quantum wire as discussed in Chapter 2 and Ref. [35. The dispersion in the left part of the wire is shifted by $V_{0}$. The energy gap due to the Zeeman effect is $E_{G}=2 \gamma B_{x}$, and the horizontal shift between $(s=+)$-branch (solid line) and the $(s=-)$-branch (dashed line) comes from the SOI.

As can be seen from Fig. 3.9 for a system with the same parameters as in Fig. 3.7 but with an additional potential step $V_{0}=0.4$, the region with perfect total conductance, i.e. $G_{\text {total }}=2 e^{2} / h$, is confined to the interval $\left(-2+\gamma B_{x}+V_{0}, 2-\gamma B_{x}\right)$ and the component oscillations in the middle region are symmetric around $\mu=V_{0} / 2$. However, the component oscillations start at $\mu_{0}=-2+\gamma B_{x}$. Thus, $\mu_{0}$ is a characteristic point for given magnetic field $B_{x}$, independent from $V_{0}, \alpha_{y}$ and $\alpha_{z}$.

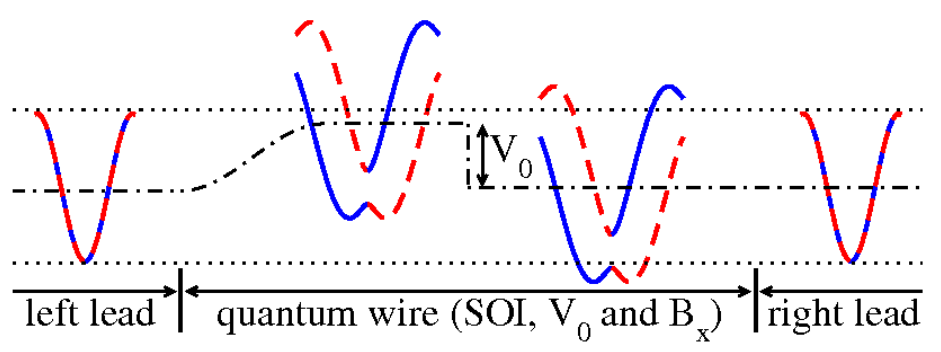

Figure 3.8: Schematic picture of the system under consideration with qualitative dispersions of the different regions. The leads have a cosine-like dispersion, whereas the dispersion in the quantum wire has two non-degenerate branches, i.e. $s=+$ (solid line) and $s=-$ (dashed line). The on-site potential structure is indicated by the dashed-dotted curve. The energy band of the leads is confined by the dotted line. 


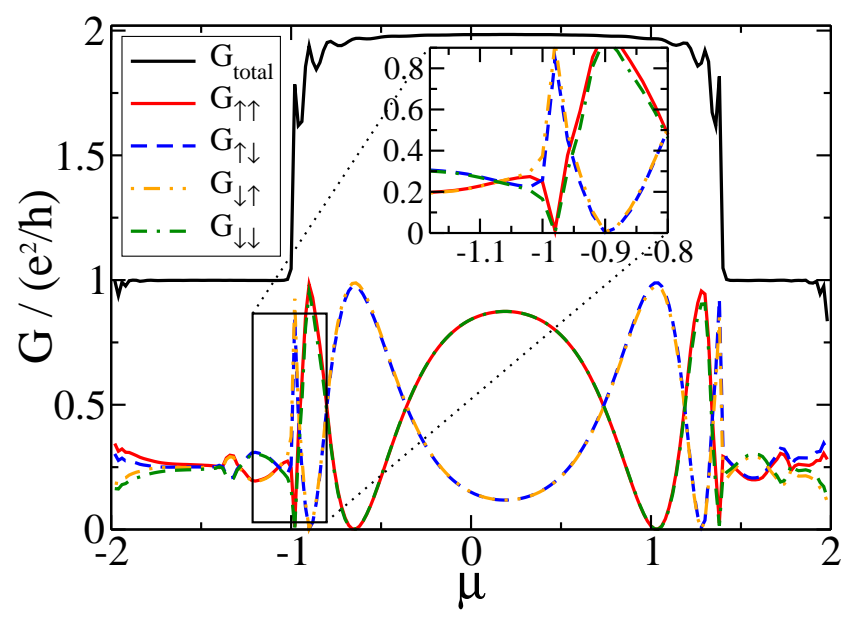

Figure 3.9: Conductance components over the chemical potential in presence of a magnetic field, SOI and a potential step with system parameters $N=100, m_{1}=20$, $t=1, t_{L}=t_{R}=1, \alpha_{y}=0, \alpha_{z}=0.8, \gamma B_{x}=0.6$ and $V_{0}=0.4$. Perfect conductance is obtained for $\mu \in\left(-2+\gamma B_{x}+V_{0}, 2-\gamma B_{x}\right)$. The inset shows the degeneracy change of the conductance components at the interface of the left region $\left(G_{\uparrow \uparrow}=G_{\downarrow \uparrow}\right.$, $\left.G_{\uparrow \downarrow}=G_{\downarrow \downarrow}\right)$ and the middle region $\left(G_{\uparrow \uparrow}=G_{\downarrow \downarrow}, G_{\uparrow \downarrow}=G_{\downarrow \uparrow}\right)$.

Another interesting feature is plotted in the inset of Fig. 3.9. In the middle region, one obtains the same degeneracy as in the case without potential step [see Fig. 3.7, i.e. $G_{\uparrow \uparrow}=G_{\downarrow \downarrow}, G_{\uparrow \downarrow}=G_{\downarrow \uparrow}$. This degeneracy is changed into $G_{\uparrow \uparrow}=G_{\downarrow \uparrow}$, $G_{\uparrow \downarrow}=G_{\downarrow \downarrow}$ at the left interface and into $G_{\uparrow \uparrow}=G_{\uparrow \downarrow}, G_{\downarrow \uparrow}=G_{\downarrow \downarrow}$ at the right interface.

In terms of simple scattering theory, the behavior of the conductance shown in this subsection can at least roughly be explained by a qualitative picture as shown in Fig. 3.8

Lets think of a single electron coming out of the left lead with energy $\mu$ close to the lower dotted line, i.e. $\mu \in(-2,-2+\delta)$. If the potential $V_{0}$ is as large as shown for clarity in Fig. 3.8 there are no propagating states in the left part of the quantum wire with this energy and the electron can only be transmitted by an evanescent wave. Indeed, we can observe such a tunnel mechanism for very short systems $(N<10)$. For $V_{0}<\gamma B_{x}$, there is always one conducting branch for small $\mu$ in both areas of the quantum wire and therefore $G_{\text {total }}=1 e^{2} / h$. For $\mu>\gamma B_{x}$, the right part of the wire provides two branches, but since the left part's dispersion is shifted by $V_{0}$, the region of perfect conductance starts at $\mu_{0}=-2+\gamma B_{x}+V_{0}$. The lower branch of the dispersion in the right part stops at $\mu_{1}=2-\gamma B_{x}$. Thus, perfect conductance is restricted to $\mu \in\left(\mu_{0}, \mu_{1}\right)$, at least for long systems in which all tunneling processes can be neglected.

The analysis of the different conductance components in this picture is much more difficult, as the $z$-component of the electron spin is not conserved within the quantum wire due to SOI and all of the spin expectation values depend on the wave number 


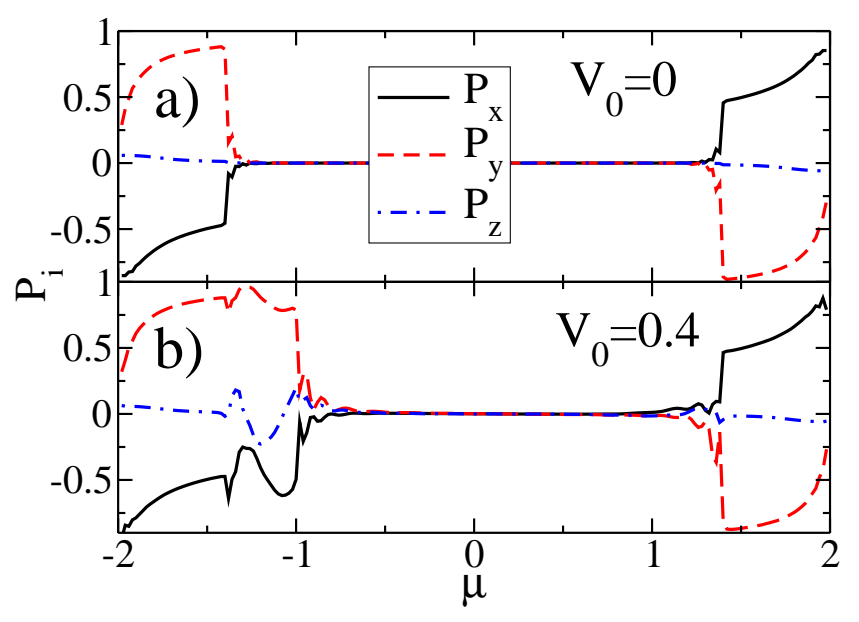

Figure 3.10: Spin polarization in $x$-, $y$ - and $z$-direction for a) a homogeneous system $\left(V_{0}=0\right)$ and b) a system with potential step $V_{0}=0.4$. The other system parameters are the same as in Fig. 3.9. Inside the region with two conducting channels, there is no significant spin polarization. The high and low energy polarization is independent from $V_{0}$ in good approximation. Only for $\mu \in\left(-2+\gamma B_{x},-2+\gamma B_{x}+V_{0}\right)$, we obtain modifications due to the interplay of SOI with the potential step.

$k$ and thus on $\mu$. One would need to solve the entire scattering problem analytically with exact eigenstates, which is a complicated but feasible task in continuum (see Ref. [26]), but impossible for the lattice setup presented here.

Instead of deepening this problem, we focus on the spin polarization. Fig. 3.10 shows the spin polarization $P_{x}, P_{y}, P_{z}$ in $x-, y$ - and $z$-direction for a homogeneous system [a) $\left.V_{0}=0\right]$ and a system with potential step [b) $\left.V_{0}=0.4\right]$. The other parameters are the same as in Fig. 3.9. The curves show a large spin polarization in $x$ - and $y$-direction for $\mu \notin\left(-2+\gamma B_{x}+V_{0}, 2-\gamma B_{x}\right)$, i.e. as long as there is only one conducting channel. This is in close analogy to the continuum case. By direct comparison of Figs. 3.10 a) and b), one observes that, at least in good approximation, each polarization component is independent from $V_{0}$ in the high-energy regime, i.e. for $\mu>2-\gamma B_{x}$, as well as in the low-energy regime, i.e. for $\mu<-2+\gamma B_{x}$. For the homogeneous system $\left(V_{0}=0\right)$, we obtain polarizations $P_{i}$ which are perfectly pointsymmetric with respect to $\left(\mu, P_{i}\right)=(0,0), i=x, y, z$. In analogy to the continuum case, the spin expectation values of the lattice system have opposite sign on the different branches $(s=+,-)$ of the energy dispersion, if the wave number $k$ is the same, i.e. $\left\langle\sigma_{i}\right\rangle_{k, s}=-\left\langle\sigma_{i}\right\rangle_{k,-s}$. Moreover, the quantum number $s$ is conserved in a homogeneous system and the energetic range (within the energy band of the leads) in which only $(s=+)$-states propagate and the range where only $(s=-)$-states propagate cover exactly the same interval in $k$-space [see Fig. 3.8 with $V_{0}=0$ ]. Therefore, we get the point-symmetry obtained in Fig. $3.10 \mathrm{a}$ ).

However, the most interesting part for our purpose is the region $\mu \in(-2+$ 
$\left.\gamma B_{x},-2+\gamma B_{x}+V_{0}\right)$ in Fig. 3.10 b), which is, up to band edge effects, the same region, where spin polarization was observed in the continuum case. In this interval of width $V_{0}$, we observe sizeable oscillations of the $P_{i}$. This also leads to finite values of $P_{z}$ which was shown to vanish for $\alpha_{y}=0$ in the continuum case. As we have pointed out in Chapter 2 and Ref. [35] for the continuum model, the polarization $P_{x}$ (in $x$-direction) only depends on the absolute value $\alpha$ and not on the direction of the effective SOI field and $\left|P_{y} / P_{z}\right|=\left|\alpha_{z} / \alpha_{y}\right|$. Averaging over the polarization oscillations, this also holds in very good approximation for the lattice model, although the lattice polarization is defined by Eq. (3.27) and not in terms of spin expectation values (see Ref. [26]). However, we emphasize that a correspondence to the continuum model can be at least qualitatively, since we investigate a different system here due to the finite length of the quantum wire and the connection to the leads matching a somewhat more realistic situation, if one thinks of experimental implementation.

However, the occurence of large oscillations means that the spin polarization reacts very sensitively to changes in the chemical potential $\mu$. Conversely, one could hope that the spin polarization can be tuned by altering the chemical potential of the system, which should be feasible in experiments by applying a gate voltage to the leads and the quantum wire itself.

\subsubsection{Single impurity}

Another interesting polarization effect occurs, if we insert a single impurity of strength $V_{1}$ in the middle of our system. This can be realized by setting $V_{j, \sigma}$ in Eq. (3.3) to $V_{1}$ for $j=[N / 2]$ and $\sigma=\uparrow, \downarrow$ and $V_{j, \sigma}=0$ for $j \neq[N / 2]$. Experimentally, there might be several possibilities to obtain $V_{1} \neq 0$. It might be difficult to contact a single lattice site (a single atom) in order to apply a gate voltage to it, but one can think of inserting an impurity atom or exciting a host atom by laser radiation in order to tune $V_{1}$ very precisely [44].

Fig. 3.11 shows the conductance and spin polarization of a system with $N=101$ lattice sites with a single impurity $V_{1}$ in the middle of the system for a magnetic field $\gamma B_{x}=0.6$ and different SOI couplings $\alpha_{z}\left(\alpha_{y}=0\right)$. The exact position of $V_{1}$ is not relevant as long as it is far away from the contacts to the leads. As can be seen from Fig. 3.11 a), the system without SOI and without any impurity $\left(V_{1}=0\right)$ shows a perfect step-like polarization in $x$-direction, whereas $P_{y}=P_{z}=0$. The spins are aligned along the direction of the magnetic field $B_{x}$, i.e. $x$-direction, and show the normal Zeeman splitting behavior. Both spin directions are possible for $\mu \in\left(-2+\gamma B_{x}, 2-\gamma B_{x}\right)$ leading to $P_{x}=0$ in this interval. Moreover, there is a range of width $\gamma B_{x}$ (starting at the band edges) in which only one spin direction is energetically possible leading to a perfect polarization, $P_{x}= \pm 1$. Choosing $\gamma B_{x}=2$, one would be able to swap $P_{x}$ between -1 and +1 just by a small variation of the chemical potential around $\mu=0$. However, we are more interested in controlling the spin arbitrarily in the sphere, defined by $P_{i} \in[0,1]$ and $\sum P_{i}^{2}=1$ with $i=x, y, z$.

Fig. $3.11 \mathrm{~b}$ ) shows that a smooth tuning of $P_{x}$ is possible in presence of a strong 


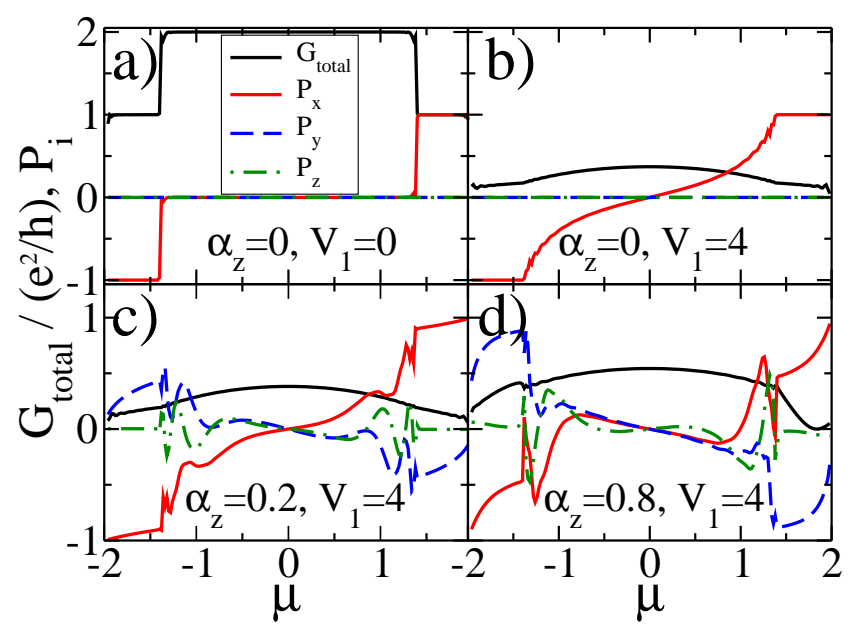

Figure 3.11: Conductance and spin polarization for a system of $N=101$ lattice sites with a single impurity $V_{1}$ in the middle of the system in presence of a magnetic field $\gamma B_{x}=0.6$ and different SOI couplings $\alpha_{z}\left(\alpha_{y}=0\right)$. a) The homogeneous system shows a perfect step-like polarization in $x$-direction. b) The shape of $P_{x}$ is washed out in presence of a strong single impurity $V_{1}=4$ and the conductance is severely decreased. c) Small SOI coupling $\alpha_{z}=0.2$ leads to finite $P_{y}, P_{z}$ and polarization oscillations. d) The oscillations are enhanced by increasing $\alpha_{z}$.

single impurity $V_{1}=4$, but the total conductance is severely decreased in this case. As $\alpha_{y}=\alpha_{z}=0$, there is no polarization in $y$ - and $z$-direction.

For small SOI, $\alpha_{z}=0.2, P_{x}$ is still the dominant polarization component, but $P_{y}$ and $P_{z}$ become finite. All polarization components show oscillations for $\mu \in$ $\left(-2+\gamma B_{x}, 2-\gamma B_{x}\right)$, which become heavily pronounced at the edges of this interval [see Fig. $3.11 \mathrm{c}$ )].

As shown in Fig. 3.11 d) for large SOI, $\alpha_{z}=0.8$, we observe the same behavior for $\mu \notin\left(-2+\gamma B_{x}, 2-\gamma B_{x}\right)$ as in the impurity-free case [see Fig. [3.10] a)] with $P_{z}$ playing only a minor role. The oscillations of the polarization components are more pronounced compared to the case with small $\alpha_{z}$, especially for $P_{x}$. Moreover, the total conductance is enlarged due to the smaller ratio of $V_{1}$ and the effective hopping $\sqrt{t^{2}+\alpha^{2}}$. Due to the occurrence of such large oscillations, each spin polarization component reacts very sensitively to changes in $\mu$ and can therefore be tuned by adjusting $\mu$.

We also observe in Fig. 3.11 that the point symmetry of each polarization component is conserved in presence of a single impurity.

If we change the direction of the magnetic field, e.g. $\mathbf{B}=(B, 0,0) \rightarrow \mathbf{B}=$ $(0,0, B)$, we, qualitatively, obtain no new results. For $\alpha_{y}=0, \alpha_{z} \neq 0$, we obtain the same conductance and $P_{x} \rightarrow P_{z}, P_{y} \rightarrow P_{y}$ and $P_{z} \rightarrow-P_{x}$, i.e. changing the direction of the magnetic field from $x$ - to $z$-direction leads to a rotation of the polarization 


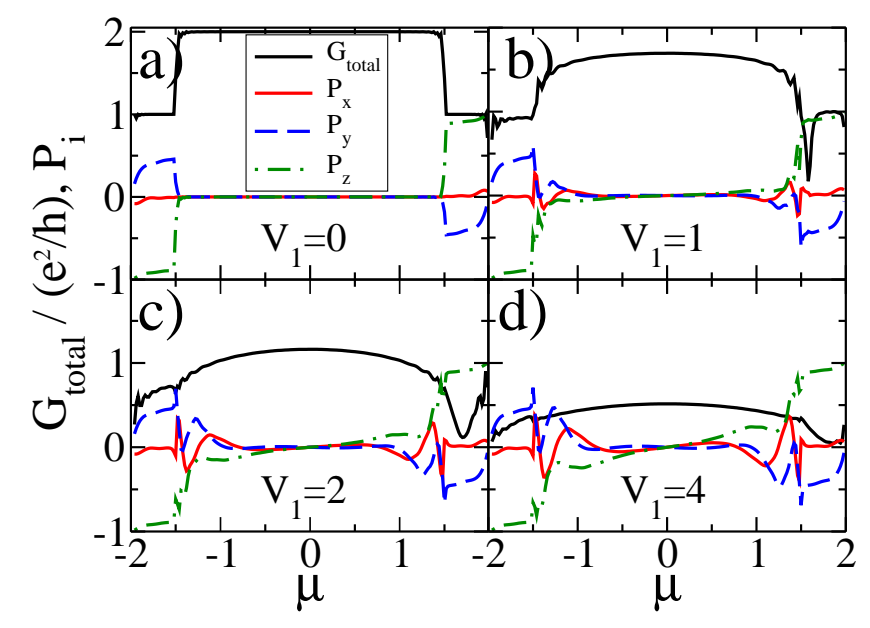

Figure 3.12: Conductance and spin polarization for a system of $N=101$ lattice sites with a single impurity $V_{1}$ in the middle of the system in presence of SOI, $\alpha_{y}=\alpha_{z}=0.5$, and a magnetic field in $z$-direction, $\gamma B_{z}=0.6$.

a) The main part of the polarization is carried by $P_{y}$ and $P_{z}$, whereas $P_{x}$ is negligible.

b) Even for small impurity strength, $V_{1}=1$, one obtains oscillations in all polarization components starting at the energy crossover between one and two coducting channels. The conductance is slightly decreased and its symmetry $G(\mu)=G(-\mu)$ is broken.

c) and d) For intermediate (strong) impurity strength, $V_{1}=2\left(V_{1}=4\right)$, the conductance is further decreased and the polarization oscillations are more pronounced leading to a non-neglibible $P_{x}$.

vector of $\pi / 2$ around the $y$-axis, independent of $\alpha_{z}$.

If, however, $\alpha_{y} \neq 0$ and $\alpha_{z}=0$, we get different results for the conductance and no link between the polarization components for $\mathbf{B}$ pointing either in $x$ - or $z$-direction. This is clear from the fact that the SOI coupling $\alpha_{y}$ causes a spin rotation in $z$ direction for electrons moving along the $x$-direction, whereas $\alpha_{z}$ rotates the spin into the $y$-direction avoiding any Zeeman splitting for $\mathbf{B}=B \mathbf{e}_{x}$ or $\mathbf{B}=B \mathbf{e}_{z}$. Another effect is that the conductance symmetry $G(\mu)=G(-\mu)$ is broken in presence of a magnetic field and an impurity, whereas the polarization symmetry $P_{i}(\mu)=-P_{i}(-\mu)$ is conserved for $i=x, y, z$.

Another example for the subtle interplay of SOI, magnetic field and single impurity is shown in Fig. 3.12 for $N=101$ lattice sites, $\alpha_{y}=\alpha_{z}=0.5$ and magnetic field in $z$-direction, $\gamma B_{z}=0.6$. In the case without impurity [see Fig. [3.12 a)], we again get a perfect step-like conductance with $G_{\text {total }}=e^{2} / h$ in the region with one conducting channel and $G_{\text {total }}=2 e^{2} / h$ in the region with two conducting channels.

The polarization components $P_{y}$ and $P_{z}$ would be of the same size without magnetic field, since $\alpha_{y}=\alpha_{z}$, but $P_{z}$ is favored here because of $\mathbf{B}=(0,0, B)$. Whereas 
the $x$-polarization $P_{x}$ is negligible in the case without impurity, it becomes sizeable for finite impurity strength $V_{1}$ due to the induced polarization oscillations. For energies close to the band edges with only one conducting channel the polarization is comparable to the impurity-free case. Thus, the polarization $\mathbf{P}=\left(P_{x}, P_{y}, P_{z}\right)$ can be tuned over quite a wide range in presence of SOI, magnetic field and single impurity by adjusting the chemical potential at the energy crossover between one and two conducting channels.

The systems under consideration in this chapter consisted of $N \sim 100$ lattice sites, which corresponds to a quantum wire length of tens of nm assuming a lattice spacing of several angstroms. This is far below the limit of $L \sim 1 \mu \mathrm{m}$ feasible in recent experiments [45. However, as long as the Coulomb interaction and therefore correlation effects are disregarded, an increase of the system size beyond $N \sim 100$ does not affect the results obtained and discussed in this chapter.

Our next aim is to include the Coulomb interaction in our model and study correlation effects influencing the linear conductance and the spin polarization. We add a local (Hubbard like) Coulomb interaction via

$$
H_{1}=\sum_{j, \sigma, \sigma^{\prime}} U_{1}(j) c_{j, \sigma^{\prime}}^{\dagger} c_{j, \sigma} c_{j, \sigma^{\prime}}^{\dagger} c_{j, \sigma^{\prime}}\left(1-\delta_{\sigma, \sigma^{\prime}}\right)
$$

and a nearest-neighbor Coulomb interaction $U_{2}$ via

$$
H_{2}=\sum_{j, \sigma, \sigma^{\prime}} U_{2}(j) c_{j+1, \sigma}^{\dagger} c_{j+1, \sigma} c_{j, \sigma^{\prime}}^{\dagger} c_{j, \sigma^{\prime}}
$$

to the Hamiltonian introduced in Eq. (3.1), where the lattice site argument $j$ in $U_{1}(j)$ and $U_{2}(j)$ allows for an inhomogeneous strength of the Coulomb interaction along the wire.

The new Hamiltonian cannot be analyzed by simple one-particle scattering theory as in this chapter, but a conceptual new approach is neccessary accounting for the many-particle behavior of the underlying physics. This new approach will be presented in the next chapter.

\subsection{Summary}

In this chapter, we constructed a lattice model which exhibits the same low-energy physics as the continuum model discussed in Chapter 2 in terms of energy dispersion and spin expectation values. However, in order to study the transport properties of this lattice model, some important modifications have been made in comparison to the situation in which transport was studied in the continuum model framework.

As the idea of infinite systems is an academical tool rather than a realistic assumption feasible in experiments, we restricted the system to a finite number of lattice sites and attached it to two semi-infinite Fermi liquid leads via hopping amplitudes $t_{L, R}$, which corresponds to an experimental situation of a finite size quantum wire connected to higher-dimensional leads by some tunnel junctions. The leads were 
modelled by a simple tight-binding Hamiltonian with conventional spin-conserving hopping amplitudes. We showed that additional SOI hopping elements in the leads and/or at the junctions can be absorbed into an effective hopping. However, a finite SOI in the leads would necessitate a different definition of spin polarization regarding the process of measuring the spin, since its orientation would depend on the position in the lead where it is measured. This would not match the experimental situation we had in mind here though and we therefore abandoned the SOI from the leads.

We chose a representation of electronical Wannier states $|j, \sigma\rangle$ in a lattice-spin base and used the Kubo formula and the Landauer-Büttiker formalism in order to define the linear conductance $G_{\sigma \sigma^{\prime}}$ in terms of transmission amplitudes $\mathcal{T}_{\sigma \sigma^{\prime}}$ with $\sigma, \sigma^{\prime}=\uparrow, \downarrow$ labelling the $z$-component of the spin in our representation. The linear conductance with respect to any other spin quantization axis could be obtained by a simple base tranformation. Since the spin is conserved in the leads, $G_{\sigma \sigma^{\prime}}$ is a measure for the transport of electrons which are injected into the system from the left lead with spin $\sigma$ and enter the right lead with spin $\sigma^{\prime}$. Thus, the dependence of $G_{\sigma \sigma^{\prime}}$ on the system parameters was used to give a detailed insight into how the spin can be manipulated and used for information transfer in this setup.

Expressing $\mathcal{T}_{\sigma \sigma^{\prime}}$ in terms of the $(1, N)$ matrix element $\mathcal{G}_{\sigma \sigma^{\prime}}(1, N)$ of the systems Green's function, we studied the dependence of $G_{\sigma \sigma^{\prime}}$ on the SOI parameters and the magnetic field. In order to allow for an analog to the energy-dependent measurements in the continuum case, we varied the overall chemical potential of the system and the leads, which defines the Fermi energy. The total linear conductance as a function of the chemical potential was shown to be seperated in regions with zero, one or two conducting channels. The size of these regions was easy to manipulate by the magnetic field, whereas the SOI parameters led to an oscillatory behavior of the different conductance components. Furthermore, we showed that spin polarization can be obtained and tuned over a wide range by adjusting the system parameters, if we insert a single impurity or a potential step into the system. Due to its feasible experimental implementation in terms of inserting an impurity atom into the quantum wire, the single impurity case might particularly be interesting for upcoming experiments .

Up to now, we have omitted any Coulomb interaction between the electrons. Due to the small spatial dimension of the mesoscopic systems under consideration, this effect certainly must be accounted for in order to give a realistic description. However, this cannot be done in the single-particle picture described so far. Conventional methods like first- or second-order perturbation theory as well as the self-consistent Hartree-Fock approximation are known to lead to artifacts, if they are applied to such systems in order to calculate correlation functions. Therefore, more subtle instruments, like renormalization group methods, are neccessary here. The functional renormalization group (fRG) method will shape up as appropriate numerical method for addressing the Coulomb interaction. We therefore present the main ideas of the fRG in the following chapter and study correlation effects on the conductance and spin polarization induced by the Coulomb interaction in Chapter [5] and [6] 


\section{Chapter 4}

\section{The functional renormalization group (fRG)}

The functional renormalization group (fRG) method is based on Wilson's renormalization group ( $\mathrm{RG}$ ) idea, which, originally, was motivated by problems of strong interaction in quantum field theory [46. However, the Wilson RG is a very general approach to interacting systems with many degrees of freedom (on different energy scales). Thus, it is possible to extend this idea to interacting many-particle quantum systems (see e.g. [8]). These systems are known to often suffer from infrared-divergencies in low dimensions leading to a breakdown of the conventional perturbation theory.

The functional integral approach to many-particle physics constitutes the basis of the fRG. One introduces a scale parameter $\Lambda$ (e.g. on the energy scale) which cuts off all modes with energy below $\Lambda$ to the free propagator in the generating functional for the single-particle irreducible $m$-particle vertex functions $\gamma_{m}$, which therefore become $\Lambda$-dependent, $\gamma_{m} \rightarrow \gamma_{m}^{\Lambda}$. Differentiating these vertex functions with respect to $\Lambda$ leads to an infinite hierarchy of differential equations, also known as fRG flow equations. Although this hierarchy needs to be truncated in practice, it is at least in principle exact. The set of coupled differential equations is integrated from $\Lambda=\infty$ down to $\Lambda \rightarrow 0$, resulting in the original cutoff-free problem, but with renormalized parameters, which account for the modifications due to interactions. In the past five years, the fRG has been applied to many different physical situations, e.g. the single-impurity Anderson model [47, which is quite a generic model of a single quantum dot coupled to leads, and a variety of quantum dot geometries [20. Moreover, characteristics of inhomogeneous Luttinger liquids manifesting as power law properties of correlation functions could be obtained with the fRG in one-dimensional quantum wires of correlated electrons [17, 18.

Our derivation of the fRG flow equations mainly follows Ref. 9]. 


\subsection{Functional integral and vertex functions}

As a very good description of the functional integral approach to many-particle physics can be found in e.g. Ref. 48, we refrain from giving a detailed introduction to this field, but start directly with the formalism needed to set up the fRG flow equations.

The continuum imaginary-time version of the grand canonical partition function of a fermionic many-particle system can be written as a functional integral

$$
\mathcal{Z}=\int \mathcal{D} \bar{\psi} \psi \exp \left\{-\frac{1}{\hbar} \int_{0}^{\beta \hbar} d \tau\left[\sum_{l} \hbar \bar{\psi}_{l}(\tau+0) \frac{d \psi_{l}(\tau)}{d \tau}+\mathcal{H}(\{\bar{\psi}\},\{\psi\})\right]\right\}
$$

with $\mathcal{H}$ being the system's Hamiltonian (with a free and an interaction part), $\psi, \bar{\psi}$ being Graßmann fields and $\tau$ being the imaginary time. The index $l$ denotes an arbitrary set of quantum numbers in which the free Hamiltonian is diagonal. The Graßmann fields obey the boundary condition $\psi_{l}(\beta \hbar)=\zeta \psi_{l}(0)$ and $\bar{\psi}_{l}(\beta \hbar)=\zeta \bar{\psi}_{l}(0)$ with $\zeta=1$ for bosons and $\zeta=-1$ for fermions. The Fourier transformed version of this integral in which the imaginary time $\tau$ is replaced by Matsubara frequencies $\omega_{n}$ obeying

$$
\omega_{n}=\frac{\pi}{\beta \hbar} \begin{cases}2 n & \text { for bosons } \\ 2 n+1 & \text { for fermions }\end{cases}
$$

is much more convenient for our purposes and Eq. (4.1) can be rewritten as

$$
\mathcal{Z}=\int \mathcal{D} \bar{\psi} \psi \exp \left(-\frac{1}{\hbar} S\right)
$$

with the action $S$ given by

$$
\begin{aligned}
S= & \sum_{l} \sum_{n}(-\hbar) \bar{\psi}_{l}\left(i \omega_{n}\right)\left[\mathcal{G}_{l}^{0}\left(i \omega_{n}\right)\right]^{-1} \psi_{l}\left(i \omega_{n}\right) \\
& +\frac{1}{\beta \hbar} \sum_{i, j} \sum_{m, n} V_{i, j} \delta_{m, n} \bar{\psi}_{i}\left(i \omega_{m}\right) \psi_{j}\left(i \omega_{n}\right) \\
& +\frac{1}{4 \beta \hbar} \sum_{i, j, k, l} \sum_{m, m^{\prime}, n, n^{\prime}} \bar{v}_{i, j, k, l} \delta_{m+m^{\prime}, n+n^{\prime}} \bar{\psi}_{i}\left(i \omega_{m}\right) \bar{\psi}_{j}\left(i \omega_{m^{\prime}}\right) \psi_{l}\left(i \omega_{n}^{\prime}\right) \psi_{k}\left(i \omega_{n}\right) \\
= & S_{0}+S_{\mathrm{int}}
\end{aligned}
$$

with $S_{0}$ being the free action and $S_{\text {int }}$ accounting for one-particle scattering, induced e.g. by an external scalar potential $V$, and two-particle interaction, induced e.g. by a Coulomb potential $\bar{v}$ for charged particles. $\mathcal{G}_{l}^{0}\left(i \omega_{n}\right)$ is the free propagator, $V_{i, j}$ is the $(i, j)$ matrix element of the external scalar potential, $\bar{v}_{i, j, k, l}$ is the antisymmetrized matrix element of the two-particle interaction and the Kronecker $\delta$ ensures energy conservation. A convergence factor $\exp \left(i \omega_{n} 0+\right)$ in the first two terms of Eq. (4.4) 
is essential in order to circumvent ill-defined expressions. However, we omit writing down this factor explicitely for convenience.

In order to write down and calculate expectation values of products of the Graßmann fields, like e.g. $m$-particle Green's functions, more easily, we define a generating functional

$$
\begin{aligned}
\mathcal{W}(\{\bar{\eta}\},\{\eta\}) & =\frac{1}{\mathcal{Z}_{0}} \int \mathcal{D} \bar{\psi} \psi \exp \left\{-\frac{1}{\hbar}\left[S_{0}+S_{\mathrm{int}}+(\bar{\psi}, \eta)+(\bar{\eta}, \psi)\right]\right\} \\
& =\left\langle\exp \left\{-\sum_{l} \sum_{n}\left[\bar{\psi}_{l}\left(i \omega_{n}\right) \eta_{l}\left(i \omega_{n}\right)+\bar{\eta}_{l}\left(i \omega_{n}\right) \psi\left(i \omega_{n}\right)\right]\right\}\right\rangle
\end{aligned}
$$

with external source fields $\eta_{l}, \bar{\eta}_{l} . \mathcal{Z}_{0}$ is the partition function of the non-interacting system represented by the action $S_{0}$. To keep the notation short, we introduce a multi-index $k=\left(\omega_{n}, l\right)$ and set $\hbar=1$. Differentiating Eq. (4.5) with respect to $\bar{\eta}$ and $\eta$ gives the $m$-particle Green's functions. Following this way, one would also account for unimportant Feynman diagrams which cancel out later on anyway.

Therefore, we define the functional

$$
\mathcal{W}^{c}(\{\bar{\eta}\},\{\eta\})=\ln [\mathcal{W}(\{\bar{\eta}\},\{\eta\})]
$$

and obtain by differentiating $\mathcal{W}^{c}$ with respect to $(\bar{\eta}, \eta)$ the connected Green's functions

$$
\begin{aligned}
G_{m}^{c}\left(k_{1}^{\prime}, \ldots, k_{m}^{\prime} ; k_{1}, \ldots, k_{m}\right) & =\zeta^{m}\left\langle\psi_{k_{1}^{\prime}} \ldots \psi_{k_{m}^{\prime}} \bar{\psi}_{k_{m}} \bar{\psi}_{k_{1}}\right\rangle_{c} \\
& =\left.\frac{\delta^{m}}{\delta \bar{\eta}_{k_{1}^{\prime}} \ldots \delta \bar{\eta}_{k_{m}^{\prime}}} \frac{\delta^{m}}{\delta \eta_{k_{m}} \ldots \delta \eta_{k_{1}}} \mathcal{W}^{c}(\{\bar{\eta}\},\{\eta\})\right|_{\eta=0=\bar{\eta}}
\end{aligned}
$$

In a final step, we take the Legendre transform of Eq. (4.6) in order to obtain a generating functional $\Gamma$ for the connected one-particle irreducible Green's functions which are the vertex functions $\gamma_{m}$ which play a central role in the fRG formalism. By introducing

$$
\phi_{k}=-\left.\frac{\delta}{\delta \bar{\eta}_{k}} \mathcal{W}^{c}(\{\bar{\eta}\},\{\eta\})\right|_{\eta=0=\bar{\eta}}
$$

and

$$
\bar{\phi}_{k}=-\left.\zeta \frac{\delta}{\delta \eta_{k}} \mathcal{W}^{c}(\{\bar{\eta}\},\{\eta\})\right|_{\eta=0=\bar{\eta}}
$$

as the new fields, we obtain

$$
\Gamma(\{\bar{\phi}\},\{\phi\})=-\mathcal{W}^{c}\left(\{\bar{\eta},\{\eta\})-(\bar{\phi}, \eta)-(\bar{\eta}, \phi)+\left(\bar{\phi},\left[\mathcal{G}^{0}\right]^{-1} \phi\right) .\right.
$$

The last summand on the rhs has been added just for later convenience. In analogy to Eq. (4.7), the vertex functions are given by

$$
\gamma_{m}\left(k_{1}^{\prime}, \ldots, k_{m}^{\prime} ; k_{1}, \ldots, k_{m}\right)=\left.\frac{\delta^{m}}{\delta \bar{\phi}_{k_{1}^{\prime}} \ldots \delta \bar{\phi}_{k_{m}^{\prime}}} \frac{\delta^{m}}{\delta \phi_{k_{m}} \ldots \delta \phi_{k_{1}}} \Gamma(\{\bar{\phi}\},\{\phi\})\right|_{\phi=0=\bar{\phi}}
$$


$\gamma_{0}$ can be interpretated as the grand canonical potential induced by the action $S_{\text {int }}$ [49]. $\gamma_{1}$ is the systems self energy $\Sigma$ (up to a sign), i.e.

$$
\gamma_{1}\left(k^{\prime}, k\right)=\zeta \Sigma_{k^{\prime}, k}
$$

with $\zeta=1(-1)$ for bosons (fermions). $\gamma_{2}$ is often referred to as effective interaction and is given by

$$
\gamma_{2}\left(k_{1}^{\prime}, k_{2}^{\prime} ; k_{1}, k_{2}\right)=-\sum_{q_{1}^{\prime}, q_{2}^{\prime}, q_{1}, q_{2}}[\mathcal{G}]_{k_{1}^{\prime}, q_{1}^{\prime}}^{-1}[\mathcal{G}]_{k_{2}^{\prime}, q_{2}^{\prime}}^{-1}[\mathcal{G}]_{q_{2}, k_{2}}^{-1}[\mathcal{G}]_{q_{1}, k_{1}}^{-1} G_{2}^{c}\left(q_{1}^{\prime}, q_{2}^{\prime} ; q_{1}, q_{2}\right)
$$

\subsection{Infrared cutoff and flow equations}

Interacting fermionic many-particle systems often suffer from analytical divergencies at the Fermi surface, particularly in low dimensions. If these divergencies occur without any counterterms of the same diagrammatic order, one can expect a breakdown of the perturbation theory in the low-energy limit. For the one-dimensional translational invariant electron gas with two-particle interaction $\tilde{v}$, an example of such a divergency can already be found for the two-particle Green's function in second order $\tilde{v}$. The divergency in this model is logarithmic and stems from the singular behavior of the free propagator in the low-energy limit. But other electronic models, especially lattice models, also suffer from this difficulty and need a special treatment.

The idea is to provide the free propagator with an infrared-cutoff parameter $\Lambda>0$ which neglects all contributions with frequency $\left|\omega_{n}\right|<\Lambda$. The easiest and most intuitive cutoff is a $\Theta$-function

$$
\mathcal{G}_{q}^{0, \Lambda}\left(i \omega_{n}\right)=\Theta\left(\left|\omega_{n}\right|-\Lambda\right) \mathcal{G}_{q}^{0}
$$

but a smooth cutoff is also conceivable and might be advantageous, depending on the special situation. Naturally, the generating functionals $\mathcal{W}, \mathcal{W}^{c}$ and $\Gamma$ now also depend on $\Lambda$.

The RG idea is to differentiate the vertex functions with respect to $\Lambda$ in order to obtain a system of coupled ordinary differential equations (ODE). The solutions of this ODE system are regular in many cases, even for $\Lambda \rightarrow 0$, and correspond to the vertex functions of the original cutoff-free model in this limit. We will only sum up the most important steps, since a detailed description of this procedure is given in Ref. [9].

We first need to differentiate $\Gamma$ with respect to $\Lambda$. For a given free propagator $\mathcal{G}^{0, \Lambda}$, we define the full propagator $\mathcal{G}^{\Lambda}$ via

$$
\mathcal{G}^{\Lambda}=\left[\left(\mathcal{G}^{0, \Lambda}\right)^{-1}-\Sigma^{\Lambda}\right]^{-1}
$$


with $\Sigma^{\Lambda}$ being the system's cutoff dependent self energy, which accounts for interaction effects. Introducing

$$
\mathcal{V}_{\bar{\phi}, \phi}\left(\Gamma^{\Lambda}, \mathcal{G}^{0, \Lambda}\right)=\left(\begin{array}{cc}
\frac{\delta^{2} \Gamma^{\Lambda}}{\delta \bar{\phi} \delta \phi}-\zeta\left(\mathcal{G}^{0, \Lambda}\right)^{-1} & \frac{\delta^{2} \Gamma^{\Lambda}}{\delta \bar{\phi} \delta \bar{\phi}} \\
\frac{\delta^{2} \Gamma^{\Lambda}}{\delta \phi \delta \phi} & \frac{\delta^{2} \Gamma^{\Lambda}}{\delta \phi \delta \bar{\phi}}-\left[\left(\mathcal{G}^{0, \Lambda}\right)^{-1}\right]^{t}
\end{array}\right)^{-1}
$$

and

$$
\tilde{\mathcal{V}}_{\bar{\phi}, \phi}\left(\Gamma^{\Lambda}, \mathcal{G}^{\Lambda}\right)=-\mathcal{V}_{\bar{\phi}, \phi}\left(\Gamma^{\Lambda}, \mathcal{G}^{0, \Lambda}\right)\left(\begin{array}{cc}
\zeta \mathcal{G}^{\Lambda} & 0 \\
0 & {\left[\mathcal{G}^{\Lambda}\right]^{t}}
\end{array}\right)^{-1}
$$

we can write

$$
\dot{\Gamma}^{\Lambda}=-\zeta \operatorname{Tr}\left(\left[\mathcal{G}^{0, \dot{\Lambda}}\right]^{-1} \mathcal{G}^{0, \Lambda}\right)+\zeta \operatorname{Tr}\left(\mathcal{G}^{\Lambda}\left[\mathcal{G}^{0, \dot{\Lambda}}\right]^{-1} \tilde{\mathcal{V}}_{\bar{\phi}, \phi}^{1,1}\left(\Gamma^{\Lambda}, \mathcal{G}^{0, \Lambda}\right)\right)
$$

whith $\tilde{\mathcal{V}}_{\bar{\phi}, \phi}^{1,1}\left(\Gamma^{\Lambda}, \mathcal{G}^{\Lambda}\right)$ denoting the $(1,1)$ element (which itself is a matrix) of $\tilde{\mathcal{V}}_{\bar{\phi}, \phi}\left(\Gamma^{\Lambda}, \mathcal{G}^{\Lambda}\right)$ Here and in the following, the dot denotes the derivative with respect to the cutoff parameter $\Lambda$. Using Eq. (4.7), we can expand $\Gamma^{\Lambda}$ in the fields $\bar{\phi}$ and $\phi$ with the vertex function $\gamma_{m}^{\Lambda}$ being the coefficient of $m$-th order, i.e.

$\Gamma^{\Lambda}(\{\bar{\phi}\},\{\phi\})=\sum_{m=0}^{\infty} \frac{\zeta^{m}}{(m !)^{2}} \sum_{k_{1}^{\prime}, \ldots, k_{m}^{\prime}} \sum_{k_{1}, \ldots, k_{m}} \gamma_{m}^{\Lambda}\left(k_{1}^{\prime}, \ldots, k_{m}^{\prime} ; k_{1}, \ldots, k_{m}\right) \bar{\phi}_{k_{1}^{\prime}} \ldots \bar{\phi}_{k_{m}^{\prime}} \phi_{k_{m}} \ldots \phi_{k_{1}}$.

Comparison of the derivative of this expression with Eq. (4.18) in each order of the fields $\bar{\phi}$ and $\phi$ yields the system of coupled differential equations for the vertex functions mentioned above. We obtain in zeroth order

$$
\dot{\gamma}_{0}^{\Lambda}=-\zeta \operatorname{Tr}\left(\left[\mathcal{G}^{0, \dot{\Lambda}}\right]^{-1} \mathcal{G}^{0, \Lambda}\right)+\zeta \operatorname{Tr}\left(\left[\mathcal{G}^{0, \dot{\Lambda}}\right]^{-1} \mathcal{G}^{\Lambda}\right),
$$

which means that the flow of $\gamma_{0}^{\Lambda}$ couples to $\gamma_{1}^{\Lambda}$. As $\gamma_{0}^{\Lambda}$ itself does not appear on the right-hand side of Eq. (4.20), this equation can directly be integrated, at least formally, but is of no further interest here.

With $\gamma_{2}^{\Lambda}\left(k^{\prime}, ; k,\right)$ denoting the matrix with elements

$$
\left[\gamma_{2}^{\Lambda}\left(k^{\prime}, ; k,\right)\right]_{q^{\prime}, q}=\gamma_{2}^{\Lambda}\left(k^{\prime}, q^{\prime} ; k, q\right),
$$

the flow equation of first order reads

$$
\dot{\gamma}_{1}^{\Lambda}\left(k^{\prime} ; k\right)=\zeta \dot{\Sigma}_{k^{\prime}, k}^{\Lambda}=\operatorname{Tr}\left(\mathcal{G}^{\Lambda}\left[\mathcal{G}^{0, \dot{\Lambda}}\right]^{-1} \mathcal{G}^{\Lambda} \gamma_{2}^{\Lambda}\left(k^{\prime}, ; k,\right)\right)
$$

Thus, the flow of $\gamma_{1}^{\Lambda}$ is coupled to itself and the two-particle vertex $\gamma_{2}^{\Lambda}$. This is diagrammatically shown in Fig. 4.1. The line with the dash corresponds to $\mathcal{G}^{\Lambda}\left(\partial_{\Lambda}\left[\mathcal{G}^{0, \Lambda}\right]^{-1}\right) \mathcal{G}^{\Lambda}$ and is often referred to as the single-scale propagator. 


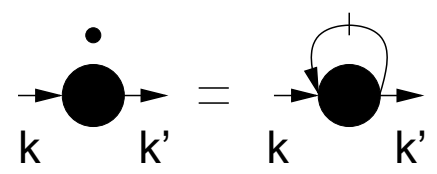

Figure 4.1: Diagrammatic representation of the flow equation for the one-particle vertex (self energy), see Eq. (4.22).
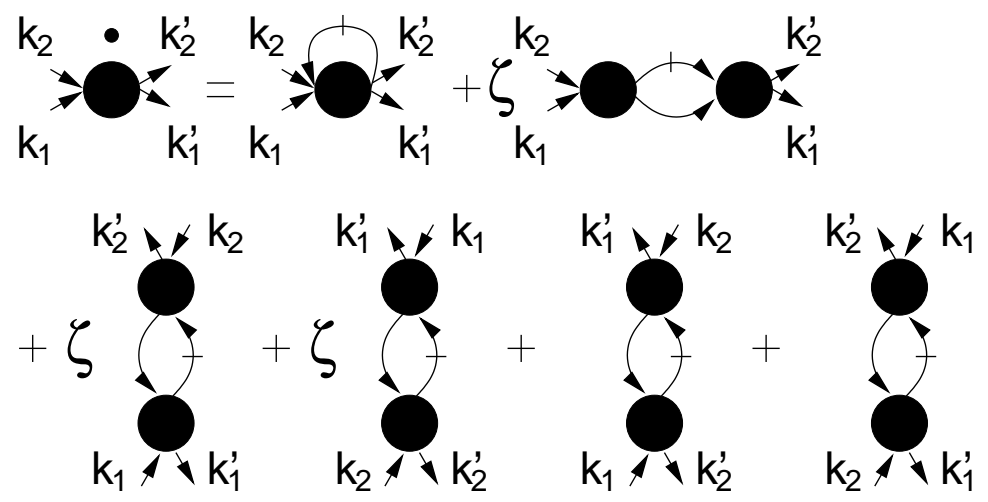

Figure 4.2: Diagrammatic representation of the flow equation for the two-particle vertex, see Eq. (4.23).

In second order, we obtain

$$
\begin{aligned}
\dot{\gamma}_{2}^{\Lambda}\left(k_{1}^{\prime}, k_{2}^{\prime} ; k_{1}, k_{2}\right)= & \operatorname{Tr}\left(\mathcal{G}^{\Lambda}\left[\mathcal{G}^{0, \dot{\Lambda}}\right]^{-1} \mathcal{G}^{\Lambda} \gamma_{3}^{\Lambda}\left(k_{1}^{\prime}, k_{2}^{\prime} ; k_{1}, k_{2},\right)\right) \\
& +\zeta \operatorname{Tr}\left(\mathcal{G}^{\Lambda}\left[\mathcal{G}^{0, \dot{\Lambda}}\right]^{-1} \mathcal{G}^{\Lambda} \gamma_{2}^{\Lambda}\left(, ; k_{1}, k_{2}\right)\left[\mathcal{G}^{\Lambda}\right]^{t} \gamma_{2}^{\Lambda}\left(k_{1}^{\prime}, k_{2}^{\prime} ;,\right)\right) \\
& +\zeta \operatorname{Tr}\left(\mathcal{G}^{\Lambda}\left[\mathcal{G}^{0, \dot{\Lambda}}\right]^{-1} \mathcal{G}^{\Lambda} \gamma_{2}^{\Lambda}\left(k_{1}^{\prime}, ; k_{1},\right) \mathcal{G}^{\Lambda} \gamma_{2}^{\Lambda}\left(k_{2}^{\prime}, ; k_{2},\right)\right. \\
& \left.+\zeta\left[k_{1}^{\prime} \leftrightarrow k_{2}^{\prime}\right]+\zeta\left[k_{1} \leftrightarrow k_{2}\right]+\left[k_{1}^{\prime} \leftrightarrow k_{2}^{\prime}, k_{1} \leftrightarrow k_{2}\right]\right),
\end{aligned}
$$

with $\left[q \leftrightarrow q^{\prime}\right]$ in the last line standing for the third line with a permutation of $q$ and $q^{\prime}$. As can be seen from Fig. 4.2, there are different contributions to the flow of $\gamma_{2}^{\Lambda}$. The first diagram in the first line of Fig. 4.2 represents the coupling to the threeparticle vertex $\gamma_{3}^{\Lambda}$, the second diagram in the first line accounts for particle-particle processes and the diagrams in the second line for particle-hole processes.

We could continue building up this hierarchy of flow equations with $\dot{\gamma}_{m}^{\Lambda}$ being coupled to $\gamma_{n}^{\Lambda}$ with $0<n \leq m+1$. This would lead to an infinite system of differential equations, which would yield the exact vertex functions in all orders. Such an infinite system is, naturally, impossible to solve. Hence, we will neglect all $m$-particle vertex functions $\gamma_{m}^{\Lambda}$ with $m \geq 3$ in the following, which has been shown by comparison to other RG methods to lead to very good results [17, 20, 43, 47, since $\gamma_{3}^{\Lambda}$ is generated only from terms of third in the two-particle vertex $\gamma_{2}^{\Lambda}$, which 
are small as long as $\gamma_{2}^{\Lambda}$ becomes not too large. Thus, we establish a closed system of coupled differential equations for $\gamma_{1}^{\Lambda}$ and $\gamma_{2}^{\Lambda}$.

In order to solve the obtained differential equations, one needs to consider a starting value $\Lambda_{0}$ for the cutoff parameter. We choose $\Lambda_{0}$ such that $\mathcal{G}^{0, \Lambda_{0}}=0$, i.e. all degrees of freedom in the free propagator are turned off at the beginning of the RG flow. We do not want to address the issue of mathematical peculiarities which can arise due to the choice of $\Lambda_{0}$, but choose a more descriptive way. Since $\mathcal{G}^{0, \Lambda_{0}}=0$, only the bare interaction vertices occur in the diagrammatic description of the $m$-particle vertex functions at $\Lambda=\Lambda_{0}$. It follows for the generating functional

$$
\Gamma^{\Lambda_{0}}(\{\bar{\phi}\},\{\phi\})=S_{\text {int }}(\{\bar{\phi}\},\{\phi\})
$$

and for the $m$-particle vertex functions

$$
\begin{aligned}
\gamma_{0}^{\Lambda_{0}} & =0 \\
\gamma_{1}^{\Lambda_{0}}\left(k^{\prime} ; k\right) & =\zeta \Sigma_{k^{\prime}, k}^{\Lambda_{0}}=\zeta V_{k^{\prime}, k} \\
\gamma_{2}^{\Lambda_{0}}\left(k_{1}^{\prime}, k_{2}^{\prime} ; k_{1}, k_{2}\right) & =\bar{v}_{k_{1}^{\prime}, k_{2}^{\prime}, k_{1}, k_{2}} \\
\gamma_{m}^{\Lambda_{0}}\left(k_{1}^{\prime}, \ldots, k_{m}^{\prime} ; k_{1}, \ldots, k_{m}\right) & =0 \text { for } m \geq 3 .
\end{aligned}
$$

Since we only account for fermions here, we set $\zeta=-1$ in the following.

\subsection{Numerical realization}

In general, it is impossible to solve the flow equations analytically. However, the computational effort to solve them numerically is quite small in the approximation we use here.

Naturally, the starting value $\Lambda_{0}=\infty$ of the cutoff parameter must be finite for any numerical algorithm. One would think intuitively that all degrees of freedom are sufficiently taken into account, if $\Lambda_{0}$ is chosen large enough. However, due to the convergence factor $\exp (i \omega 0+)$ mentioned shortly in the discussion of Eq. (4.3) we obtain a finite contribution $c_{m}^{\Lambda_{0}}$ by integrating the flow equation for $\gamma_{m}^{\Lambda}$ over $\Lambda$ from infinity down to $\Lambda_{0}$. As carried out in great detail in e.g. Refs. [43, 50, this contribution is given in first order by

$$
c_{1}^{\Lambda_{0}}\left(k^{\prime} ; k\right)=-\frac{1}{2} \sum_{q} \bar{v}_{k^{\prime}, q, k, q} .
$$

We thus obtain

$$
\begin{aligned}
\gamma_{1}^{\Lambda_{0}}\left(k^{\prime} ; k\right) & =-\Sigma_{k^{\prime}, k}^{\Lambda_{0}}=-V_{k^{\prime}, k}-\frac{1}{2} \sum_{q} \bar{v}_{k^{\prime}, q, k, q} \\
\gamma_{2}^{\Lambda_{0}}\left(k_{1}^{\prime}, k_{2}^{\prime} ; k_{1}, k_{2}\right) & =\bar{v}_{k_{1}^{\prime}, k_{2}^{\prime}, k_{1}, k_{2}},
\end{aligned}
$$

since the starting condition for the two-particle vertex $\gamma_{2}^{\Lambda}$ is not affected by this integration. 
Following e.g. Ref. 43, we neglect the frequency dependence of $\gamma_{2}^{\Lambda}$. As a consequence, $\gamma_{1}^{\Lambda}$ also becomes frequency independent. Since the bare interaction is frequency independent by definition, this approximation leads to errors in second order of the interaction strength for the self energy and in third order for the twoparticle vertex function on the Fermi surface. It has been shown in Refs. 20, 43 ] that all questions addressed here regarding the low-energy properties of the systems under consideration in this thesis can be captured with this simplification not only qualitatively, but also quantitatively with high accuracy.

We therefore carry out the frequency integration in Eqs. (4.22), (4.23) and obtain

$$
\dot{\gamma}_{1}^{\Lambda}=-\Sigma^{\Lambda}=\frac{1}{2 \pi} \sum_{\omega= \pm \Lambda} \sum_{2,2^{\prime}} \tilde{\mathcal{G}}_{2,2^{\prime}}^{\Lambda}(i \omega) \gamma_{2}^{\Lambda}\left(1^{\prime}, 2^{\prime} ; 1,2\right)
$$

and

$$
\begin{aligned}
& \dot{\gamma}_{2}^{\Lambda}\left(1^{\prime}, 2^{\prime} ; 1,2\right)=\frac{1}{2 \pi} \sum_{\omega= \pm \Lambda} \sum_{3,3^{\prime}} \sum_{4,4^{\prime}}\left[\frac{1}{2} \tilde{\mathcal{G}}_{3,3^{\prime}}^{\Lambda}(i \omega) \tilde{\mathcal{G}}_{4,4^{\prime}}^{\Lambda}(-i \omega) \gamma_{2}^{\Lambda}\left(1^{\prime}, 2^{\prime} ; 3,4\right) \gamma_{2}^{\Lambda}\left(3^{\prime}, 4^{\prime} ; 1,2\right)\right. \\
& \left.\quad+\tilde{\mathcal{G}}_{3,3^{\prime}}^{\Lambda}(i \omega) \tilde{\mathcal{G}}_{4,4^{\prime}}^{\Lambda}(i \omega)\left(-\gamma_{2}^{\Lambda}\left(1^{\prime}, 4^{\prime} ; 1,3\right) \gamma_{2}^{\Lambda}\left(3^{\prime}, 2^{\prime} ; 4,2\right)+\gamma_{2}^{\Lambda}\left(2^{\prime}, 4^{\prime} ; 1,3\right) \gamma_{2}^{\Lambda}\left(3^{\prime}, 1^{\prime} ; 4,2\right)\right)\right]
\end{aligned}
$$

with the indices 1,2 etc. representing a full set of quantum numbers for singleparticle states (e.g. in a lattice site and spin base) without frequencies. The propagator $\tilde{\mathcal{G}}^{\Lambda}$ is defined by a Dyson equation with a frequency independent self energy

$$
\tilde{\mathcal{G}}^{\Lambda}(i \omega)=\left[\mathcal{G}_{0}^{-1}(i \omega)-\Sigma^{\Lambda}\right]^{-1} .
$$

A standard numerical algorithm to solve these flow equations works as follows. One starts by setting up the starting condition according to Eq. (4.27) at $\Lambda=\Lambda_{0}$ and initializes the inverse $\left(\tilde{\mathcal{G}}^{\Lambda}\left(i \Lambda_{0}\right)\right)^{-1}=\left(\mathcal{G}_{0}^{\Lambda}\right)^{-1}\left(i \Lambda_{0}\right)-\Sigma^{\Lambda_{0}}$ of the full propagator given by Eq. (4.30) and obtains the full propagator itself by inversion of that expression. Note that this inversion can be done by an $\mathcal{O}(N)$-algorithm even in presence of SOI and magnetic field [see App. B. The result for $\tilde{\mathcal{G}}$ is inserted into Eqs. (4.28) and (4.29) giving the derivative of $\gamma_{2}^{\Lambda=\Lambda_{0}}$ and $\Sigma^{\Lambda=\Lambda_{0}}$. With an adaptive step size algorithm (e.g. Runge-Kutta or Adams algorithm [51]), a new $\Lambda_{1}<\Lambda_{0}$ is chosen, depending on the value of $\dot{\Sigma} \Lambda=\Lambda_{0}$ and $\dot{\gamma}_{2}^{\Lambda=\Lambda_{0}}$. One initializes $\left(\tilde{\mathcal{G}}^{\Lambda_{1}}\right)^{-1}$, achieves the new propagator and calculates $\dot{\Sigma}^{\Lambda=\Lambda_{1}}$ and $\dot{\gamma}_{2}^{\Lambda=\Lambda_{1}}$. This procedure is repeated until $\Lambda_{n}=0$.

All modifications of the system parameters due to the one-particle interaction $V$ and the two-particle interaction $\bar{v}$ are contained in the self energy $\Sigma^{\Lambda=0}$ at the end of the fRG flow and one is left with an interaction-free renormalized model.

Using the Dyson equation Eq. (4.30) with $\omega=\Lambda=0$ thereafter, one obtains the full propagator of the effective model and can insert it into Eq. (3.23) in order to get the linear conductance and the spin polarization. Moreover, one can calculate 
the expectation value of the particle number $\left\langle n_{j, \sigma}\right\rangle \in[0,1]$ of electrons at lattice site $j$ with spin $\sigma$. According to

$$
\left\langle n_{j, \sigma}\right\rangle=\frac{1}{2 \pi} \int_{-\infty}^{\infty} d \omega \tilde{\mathcal{G}}_{\sigma \sigma^{\prime}}(i \omega ; j, j) \exp (i \omega \delta) \delta_{\sigma \sigma^{\prime}} \stackrel{\delta \rightarrow 0}{=} \frac{1}{2}-\frac{1}{\pi} \int_{0}^{\Lambda_{0}} d \omega \Re\left[\tilde{\mathcal{G}}_{\sigma \sigma}(i \omega ; j, j)\right],
$$

one simply has to integrate the diagonal elements of the full propagator over $\omega$. Again, $\Lambda_{0}$ is a large but finite value and the summand $1 / 2$ accounts for the contribution of the integration over $\left[\Lambda_{0}, \infty\right)$. The average particle number (the filling of the quantum wire) is given by the sum

$$
\langle n\rangle=\frac{1}{N} \sum_{j=1}^{N} \sum_{\sigma=\uparrow, \downarrow}\left\langle n_{j, \sigma}\right\rangle .
$$

Note that we can calculate $\left\langle n_{j, \sigma}\right\rangle$ just approximately with Eq. (4.31), since we do not know $\tilde{\mathcal{G}}_{\sigma \sigma^{\prime}}(i \omega ; j, j)$ exactly. Another approach to obtain the local particle number $\left\langle n_{j, \sigma}\right\rangle$ is to define it via a generating functional (e.g. the grand canonical potential) and derive a separate flow equation for the density response vertex. This approach is superior to our approximation regarding e.g. the prediction of the powerlaw decay of the amplitude of Friedel oscillations in presence of impurities in the quantum wire, but reveals artifacts like $\left\langle n_{j, \sigma}\right\rangle>1$ or $\left\langle n_{j, \sigma}\right\rangle<0$ in certain parameter regimes (especially for quantum dots), in which our approach still leads to correct values. However, the average particle number of the quantum wire is correct within both approximations as has been pointed out in Ref. 43. The same holds for the frequencies of Friedel oscillations in presence of impurities in the wire. Since we will not address the power-law decay of the amplitude of the Friedel oscillations, but are more interested in a correct prediction of the local particle number for quantum dots, we will use Eqs. (4.31) and (4.32) in the following.

\subsection{Summary}

In this chapter, we presented the main idea of the functional renormalization group method. Starting from a functional integral representation for the grand canonical partition function of a general fermionic many-particle system, we derived a generating functional for the connected one-particle irreducible vertex functions. The first-order vertex function is the system's self energy (up to a sign) and the second-order vertex function can be understood as effective two-particle (Coulomb) interaction.

In order to avoid low-energy divergencies at the Fermi surface, which often occur in interacting fermionic many-particle systems, especially in low dimensions, we introduced an infrared-cutoff $\Lambda>0$ to the free propagator of the system suppressing all energy scales below $\Lambda$. The full propagator, defined by the Dyson equation, and the vertex functions therefore became $\Lambda$-dependent quantities. Deriving the vertex 
functions with respect to $\Lambda$ and setting all vertex functions of third and higher order to zero led to a closed system of differential equations.

Starting from a large but finite initial value $\Lambda_{0}$, we are able to integrate the flow equations down to $\Lambda=0$ numerically and account for all interaction-induced modifications of the system properties by considering the finite self energy $\Sigma^{\Lambda=0}$ of the system. At the end of the fRG flow $(\Lambda=0)$, one again obtains a singleparticle picture, but with renormalized system parameters (conventional hopping, SOI, magnetic field etc.).

Note, however, that this procedure also is just an approximation. But since contributions of diagrams of higher (than first) order are contained in the self energy, this method is justified to be thought of being superior to first-order perturbation theory and also to the self-consistend Hartree-Fock approximation, which leads to artifacts like charge density waves in presence of a single impurity in the quantum wire.

The fRG method avoids these artifacts and, furthermore, it is a very fast computational tool for studying correlation effects even regarding large systems and thus appropriate to investigate the behavior of interacting electrons in quantum wires with SOI and magnetic field. 


\section{Chapter 5}

\section{Quantum wires of correlated electrons with SOI and magnetic field}

Electron correlations in one-dimensional quantum systems induced by the Coulomb interaction are a very subtle topic of research in modern condensed matter physics [52. Especially in the past 15 years, much effort has been spent on developing numerical methods in order to get insight into the physics of such systems in which conventional perturbation theory breaks down.

Similarly to the non-interacting case [see Fig. 3.2], the system is connected to two semi-infinite non-interacting leads via hopping amplitudes $t_{L}$ abd $t_{R}$. The conventional hopping $t$ and the SOI parameters $\alpha_{y}, \alpha_{z}$ also have the same meaning as before. Whenever two electrons are at the same lattice site, they naturally must have opposite spin due to Pauli's exclusion principle and are repelled from each other by the repulsive local Coulomb interaction $U_{1}>0$. Electrons being located at neighboring lattice sites feel the repulsive nearest-neighbor Coulomb interaction $U_{2}>0$, independent of their spin [see Fig. 5.1].

As the continuum Coulomb interaction potential between two electrons decreases just with $1 / r$ ( $r$ being the distance between the two electrons), in contrast to the

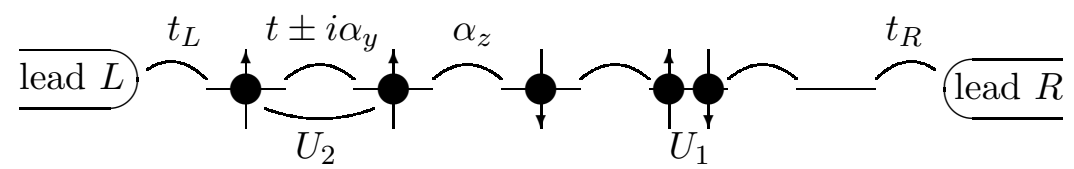

Figure 5.1: Linear geometry of quantum wire with SOI and local $\left(U_{1}\right)$ and nearestneighbor $\left(U_{2}\right)$ Coulomb interaction connected to two semi-infinite non-interacting leads via hopping amplitudes $t_{L}, t_{R}$. 
different hopping parameters which are thought of being induced by tunneling processes and, therefore, decay exponentially with $r$, one could argue that one would still have a finite repulsion induced by next-nearest-neighbor interaction $U_{3}$ and so on. However, we consider these interactions to be negligible, since they might be extremely weak in reality due to screening effects of the electron charges, and fundamental correlation effects are obtained already with implementation of $U_{1}$ and $U_{2}$.

We add the Coulomb interaction to our system via the Hamiltonians

$$
H_{1}=\sum_{j, \sigma, \sigma^{\prime}} U_{1}(j) c_{j, \sigma}^{\dagger} c_{j, \sigma} c_{j, \sigma^{\prime}}^{\dagger} c_{j, \sigma^{\prime}}\left(1-\delta_{\sigma, \sigma^{\prime}}\right)
$$

for the local part $U_{1}$ and

$$
H_{2}=\sum_{j, \sigma, \sigma^{\prime}} U_{2}(j) c_{j+1, \sigma}^{\dagger} c_{j+1, \sigma} c_{j, \sigma^{\prime}}^{\dagger} c_{j, \sigma^{\prime}}
$$

for the nearest-neighbor part $U_{2}$. Like the other system parameters, $U_{1}(j)$ and $U_{2}(j)$ are also given in units of the lead hopping $\tilde{t}_{L}=\tilde{t}_{R}$ and the lattice site argument $j$ will enable us to turn on/off the interaction smoothly at the lead contacts to avoid any unintended backscattering effects.

We apply the fRG method presented in Chapter 4 to treat correlation effects ending up with an effective non-interacting model with the renormalized parameters being included in the system's self energy $\Sigma^{\Lambda=0}$ at the end of the fRG flow.

As the flow equations of the two-particle vertex $\gamma_{2}$ derived in Ref. [18] rest upon the spin rotational invariance, we cannot apply them in our context with finite SOI. However, the flow of $\gamma_{2}$ can be neglected for short quantum wires of $N \sim \mathcal{O}\left(10^{2}\right)$ lattice sites, if $U_{1}$ and $U_{2}$ do not become too large. Therefore, in Sect. [5.1 we first investigate, how the linear conductance and the spin polarization in short systems such as in Sect. 3.4 are altered due to the Coulomb interaction. Since we obtain an effective interaction-free model at the end of the fRG flow, we can use Eqs. (3.22) and (3.23) as before (but with a modified Green's function) to calculate the conductance components $G_{\sigma \sigma^{\prime}}$.

In Sect. 5.2. we then address long quantum wires with impurities which are known to reveal characteristics of inhomogeneous Luttinger liquids in terms of power laws of correlation functions. In principal, the flow of $\gamma_{2}$ must be accounted for, but tuning the $2 k_{F}$-backscattering component $g_{1, \perp}$ to zero by choosing a special ratio $U_{1} / U_{2}$ for given $\mu$, we can suppress the importance of the flow of $\gamma_{2}$. The details of this technical trick will also be discussed in Sect. 5.2. We will then analyze the influence of the SOI on the power-law scaling of the linear conductance as a function of the system size in presence of impurities and show whether or not the spin polarization reveals a similar power-law behavior. 


\subsection{Transport properties of short quantum wires with SOI, magnetic field and Coulomb interaction}

It has been shown in Fig. 3.4 for the non-interacting case that sharply turning on/off the system parameters like the SOI $\alpha_{y}$ and $\alpha_{z}$ leads to severe oscillations of the conductance components as a function of the chemical potential $\mu$ and a decrease of the total conductance towards the band edges. These oscillations are increased for finite Coulomb interaction $U_{1}$ and $U_{2}$, as the SOI parameters represent hopping impurities which are effectively enlarged by the Coulomb interaction and the terms with $U_{1}$ and $U_{2}$ themselves can be seen as impurities if sharply turned on/off [17. However, we can entirely suppress these oscillations by turning on/off the Coulomb interaction (and accordingly the other system parameters) over $m_{1}$ lattice sites with the smooth weight function defined in Eq. (3.15). We observe that $m_{1}=20$ is sufficient in order to obtain a perfect conductance $G_{\text {total }}=2 e^{2} / h$ over the entire band for the impurity-free system of up to $N=400$ lattice sites. If not explicitely stated otherwise, we therefore choose $m_{1}=20$ in the following.

In order to get a first insight into how the Coulomb interaction alters the system's transport properties, Fig. 5.2 shows the evolution of the magnetic field $\gamma B_{x}$, the conventional hopping $t$ and the SOI $\alpha_{y}$ and $\alpha_{z}$ as functions of the flow parameter $\Lambda$ during the fRG flow for a system with $N=100$ at $\mu=0$. The Coulomb interaction is $U_{1}=1$ and $U_{2}=0.5$ and we choose start parameters $\gamma B_{x}\left(\Lambda_{0}\right)=0.6, t\left(\Lambda_{0}\right)=1$, $\alpha_{y}\left(\Lambda_{0}\right)=0.3$ and $\alpha_{z}\left(\Lambda_{0}\right)=0.5$. As expected, the important renormalization of all parameters takes place in quite a small interval $\Lambda \in(0.1,10)$ (compared to the entire flow), which is roughly confined by the bandwidth and the inverse system size.

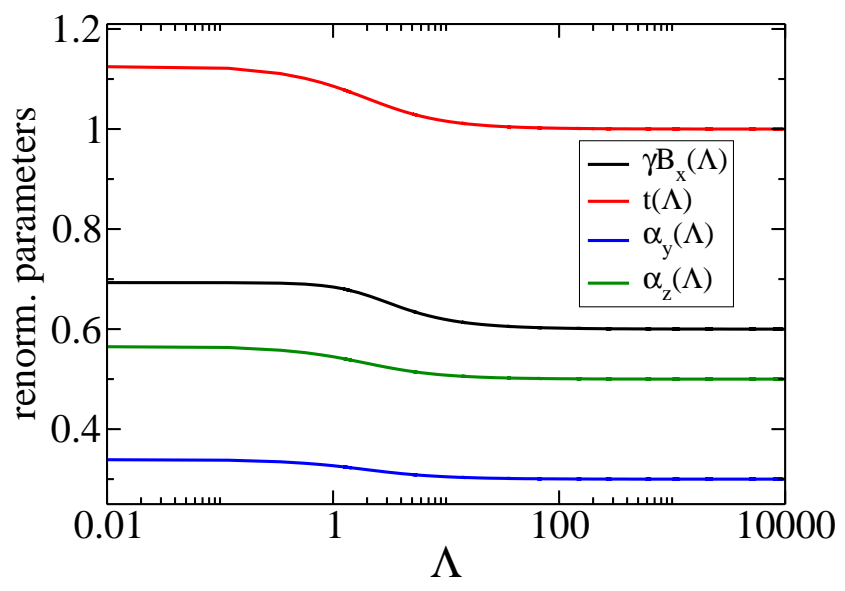

Figure 5.2: Renormalization of the system parameters as a function of the flow parameter $\Lambda$ during the fRG flow for a system with $N=100$ and interactions $U_{1}=1, U_{2}=0.5$ at $\mu=0$. The start parameters are $\gamma B_{x}\left(\Lambda_{0}\right)=0.6, t\left(\Lambda_{0}\right)=1$, $\alpha_{y}\left(\Lambda_{0}\right)=0.3$ and $\alpha_{z}\left(\Lambda_{0}\right)=0.5$. 
We emphasize that for the setup chosen in Fig. 5.2 the hopping parameters $t$, $\alpha_{y}$ and $\alpha_{z}$ are renormalized by the nearest-neighbor interaction $U_{2}$ only, whereas the magnetic field $\gamma B_{x}$ being an on-site property of the system is renormalized by $U_{1}$. However, the renormalization behavior of the system parameters becomes much more complex in presence of impurities, but is not within our scope here. Instead, we concentrate on how the linear conductance and the spin polarization are altered due to the Coulomb interaction in presence of impurities.

\subsubsection{Potential step}

A comparison of the original system parameters and the renormalized system parameters (at the end of the fRG flow at $\Lambda=0$ ) as a function of the lattice site index $j$ in presence of a potential step $V_{0}=1.5$ is shown in Fig. 5.3 for a system with $N=100$ lattice sites, chemical potential $\mu=0$ and Coulomb interactions $U_{1}=1$ and $U_{2}=0.5$. The original system parameters $t=1, \alpha_{y}=\alpha_{z}=0.5$, $\gamma B_{x}=0.6$ are turned on/off adiabatically over $m_{1}=20$ lattice sites. The original spin-degeneracy of the potential $V_{0}$, i.e. $V_{0, \uparrow}(j)=V_{0, \downarrow}(j)$, is lifted during the fRG flow leading to $V_{0, \uparrow}(j) \neq V_{0, \downarrow}(j)$. Similarly, we obtain $\alpha_{y}(j) \neq \alpha_{z}(j)$ at the end of the flow, although these two parameters coincided originally. The potential step is dramatically decreased due to the influence of the Coulomb interaction, whereas the other system parameters are increased. Moreover, a slight dependence on the lattice site index $j$ is induced for all system parameters due to the potential step. This dependence increases with increasing $V_{0}$ and increasing $U_{1}$ and $U_{2}$ and cannot be observed within first-order perturbation theory.

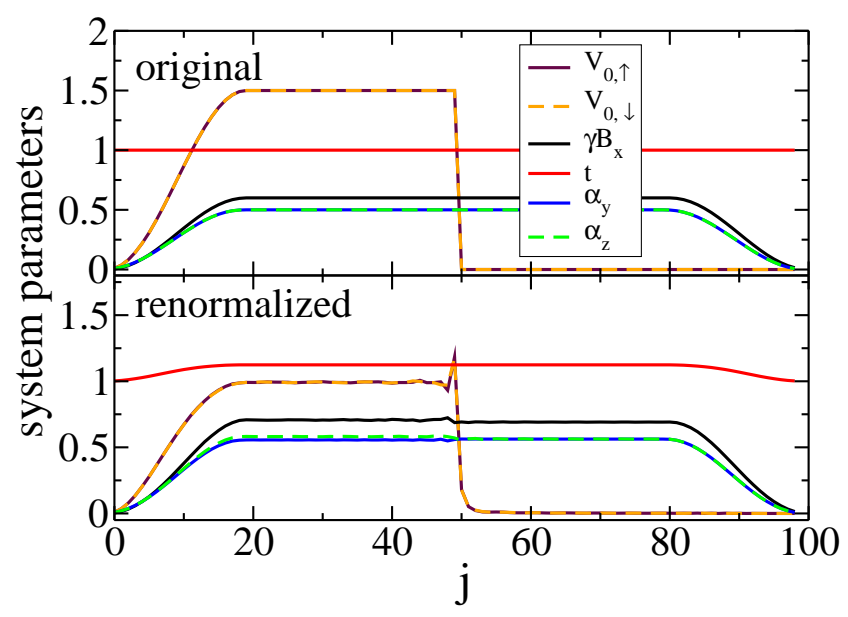

Figure 5.3: Comparison of the original and the renormalized system parameters as a function of lattice site index $j$ in presence of a potential step $V_{0}=1.5$ for a system with $N=100$ lattice sites, chemical potential $\mu=0$ and interactions $U_{1}=1$ and $U_{2}=0.5$. The original system parameters $\alpha_{y}=\alpha_{z}=0.5, \gamma B_{x}=0.6$ are turned on/off adiabatically over $m_{1}=20$ lattice sites, whereas $t=1$ is constant to match the boundary condition (with respect to the leads). 


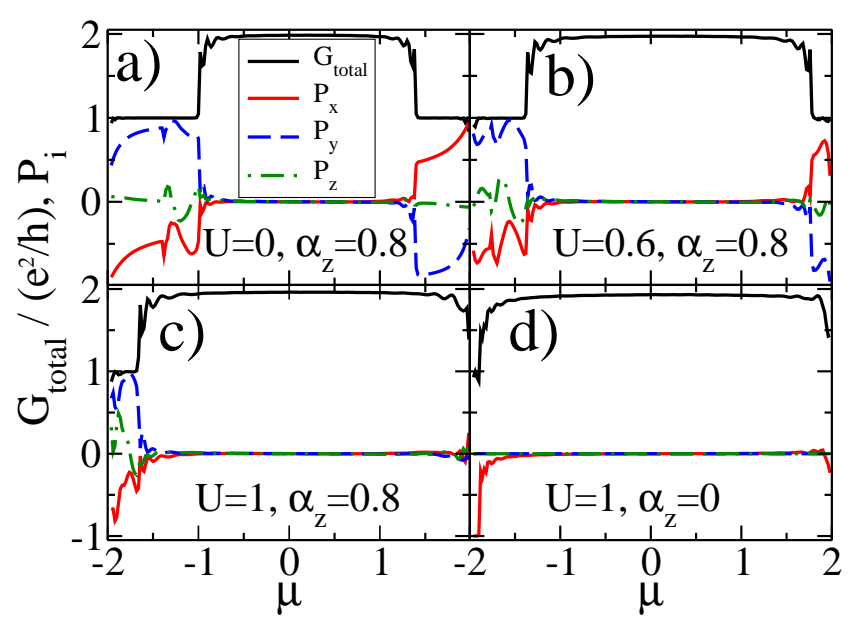

Figure 5.4: Total conductance and spin polarization over the chemical potential $\mu$ for a system with $N=100$ lattice sites, potential step $V_{0}=0.4$, magnetic field $\gamma B_{x}=0.6$ and different Coulomb interaction $U=U_{1}=2 U_{2}$.

a)-c) Increasing the Coulomb interaction for constant SOI $\alpha_{z}=0.8\left(\alpha_{y}=0\right)$ leads to a broadening of the middle part with two open conducting channels. The spin polarization vanishes in this area, but reveals a strong dependence on $\mu$ in the area with only one conducting channel.

d) For $\alpha_{z}=0$ and $U=1$, there is just a very small area with only one conducting channel left at the lower band edge, where the spin is completely polarized in $x$ direction.

Fig. [5.4 shows the total conductance and spin polarization over the chemical potential $\mu$ for a system with $N=100$ lattice sites, potential step $V_{0}=0.4$ and magnetic field $\gamma B_{x}=0.6$. As in the non-interacting case, the scalar potential is turned on adiabatically over $m_{1}$ lattice sites up to its maximum value $V_{0}$ and is sharply turned off in the middle of the system. We consider a constant ratio of local and nearest-neighbor interaction, $U_{1} / U_{2}=2$, call the local interaction $U_{1}$ simply the interaction $U$ and study its influence on $G_{\text {total }}$ and $P_{i}, i=x, y, z$. Comparing Fig. 5.4 a)-c), where the SOI parameters are $\alpha_{y}=0$ and $\alpha_{z}=0.8$, one notes that an increase of $U$ leads to a broadening of the middle part with two open conducting channels with $G_{\text {total }} \sim 2 e^{2} / h$ and $P_{i}=0$. However, we are most interested in the parameter regime which leads to a finite spin polarization. For large Coulomb interaction $U$, spin polarization can only be obtained for a chemical potential close to the lower band edge. In this range, the $P_{i}$ reveal a strong dependence on $\mu$ and can therefore be tuned over a wide range by only a small modification of $\mu$. Note that for large magnetic fields one can also observe a decrease of the middle region's width with increasing $U$. Thus, one can argue that there should be a critical magnetic field $\gamma B_{x}^{c}$ for which the division into band intervals with one and two conducting channels is independent of $U$. However, this critical field is very sensitive to the other system 
parameters and we do not want to deepen this aspect here.

For vanishing SOI, $\alpha_{y}=\alpha_{z}=0$, the range with two conducting channels ranges almost over the entire bandwidth for $U=1$ [see Fig. [5.4 d)] leaving only a narrow interval at the lower band edge in which spin polarization can be achieved. However, as there is no SOI, the spin polarization can not be tuned by changing $\mu$, but the spin is completely polarized antiparallel to the magnetic field, $P_{x}=-1$.

This shows that even in presence of Coulomb interaction the SOI can be used in order to gain control over the spin by modifying the chemical potential of a system with potential step.

\subsubsection{Potential barrier}

We now study the dependence of the conductance and the spin polarization on the Coulomb interaction for a system with a potential barrier realized by a single impurity $V_{1}$ in the middle of the system.

Fig. 5.5 shows the total conductance $G_{\text {total }}$ and polarization components $P_{i}, i=$ $x, y, z$, for a system with $N=101$ lattice sites, SOI parameters $\alpha_{y}=\alpha_{z}=0.5$ and magnetic field $\gamma B_{x}=0.6$ in $x$-direction for $V_{1}=0$ [see Fig. [5.5] a), b) ] and a single impurity in the middle of the system, $V_{1}=2$ [see Fig. [5.5 c), d) ]. We see in the impurity-free case that the range with two conducting branches, i.e. $G_{\text {total }}=$ $2 e^{2} / h$, is enlarged with increasing Coulomb interaction. Consequently, the energy range in which we can obtain spin polarization shrinks. Note that one can also obtain parameter combinations, e.g. large magnetic fields and $U_{2}=0$, where the opposite behavior is observed. This is due to the subtle interplay of the different parameters in the fRG flow leading to renormalized parameters which can not always be predicted a priori, not even qualitatively. For the parameters in Fig. 5.5 however, we could extend the range with two conducting channels over the whole energy band of the leads, i.e. $\mu \in(-2,2)$, by further increasing $U$. This would lead to a total suppression of the polarization.

Comparing Fig. 5.5 c) and d), one realizes that the Coulomb interaction further reduces the total conductance in presence of a single impurity and the area of sizeable polarization shrinks due to the enlargement of the area with two conducting channels. The polarization oscillations induced by the impurity start at the new interface of the areas with one and two conducting channels, but are altered only quantitatively. Moreover, the point symmetry $P_{i}(\mu)=-P_{i}(-\mu)$ with $i=x, y, z$ observed in the non-interacting case is destroyed by the Coulomb interaction.

\section{The way towards long wires}

It has been argued in Refs. 43, 53 that one has to be careful in applying the fRG method to large systems of correlated electrons without taking the flow of the twoparticle vertex into account. For arbitrary interactions $U_{1}, U_{2}$ and filling $n$ (chemical potential $\mu$ ), the flow of the two-particle vertex must be kept at least in lowest (that is second) order to obtain the scaling behavior of correlation functions to leading 


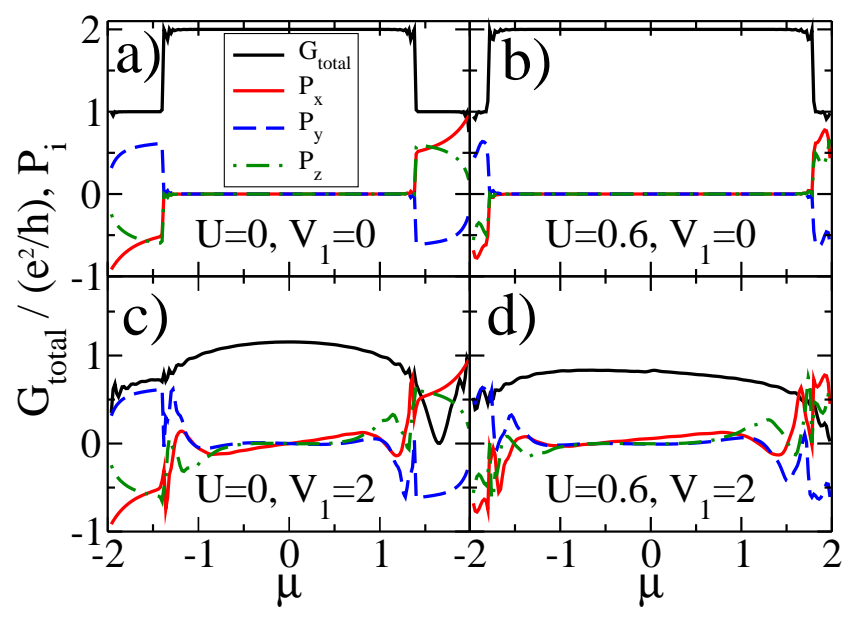

Figure 5.5: Total conductance and spin polarization components for a system with $N=101$ lattice sites, SOI coupling parameters $\alpha_{y}=\alpha_{z}=0.5$ and magnetic field in $x$-direction, $\gamma B_{x}=0.6$.

a) The conductance and spin polarization in the impurity-free non-interacting case shows the typical step-like behavior.

b) The energy range with two conducting channels, $G_{\text {total }}=2 e^{2} / h$, is enlarged with increasing Coulomb interaction, $U=U_{1}=0.6, U_{2}=0.3$.

c) A single impurity $V_{1}=2$ in the middle of the system for the non-interacting case reduces the conductance and leads to polarization oscillations.

d) In presence of Coulomb interaction, $U=U_{1}=0.6, U_{2}=0.3$, the conductance is further decreased and the polarization oscillations are altered quantitatively.

order in the interaction for systems with large $N$.

In the absence of SOI, this is already known from the so-called "g-ology" model [54. It was shown by an RG analysis of this model that the two-particle scattering of electrons with opposite spin and momentum transfer $2 k_{F}$, the so-called $g_{1, \perp}$ term given (for the extended Hubbard model) by

$$
g_{1 \perp}=U_{1}+2 \cos \left(2 k_{F}\right) U_{2}=U_{1}+\left(\mu^{2}-2\right) U_{2},
$$

flows to zero. For large $N$, this scaling must be accounted for in order to obtain correct results (in first order) for the correlation functions like e.g. the linear conductance. Since we cannot use the parametrization of the flow of the two-particle vertex proposed in Ref. [18], which is based on spin conservation, we choose a different approach here.

We choose the local and nearest-neighbor interaction $U_{1}$ and $U_{2}$ at a given chemical potential $\mu$ such that $g_{1 \perp}=0$ at the beginning of the fRG flow. If it is zero initially, it will not be generated in a RG analysis of the corresponding "g-ology" model (for vanishing SOI). Being interested in the behavior of correlation functions in leading order in the interaction, one can neglect the flow of the two-particle al- 


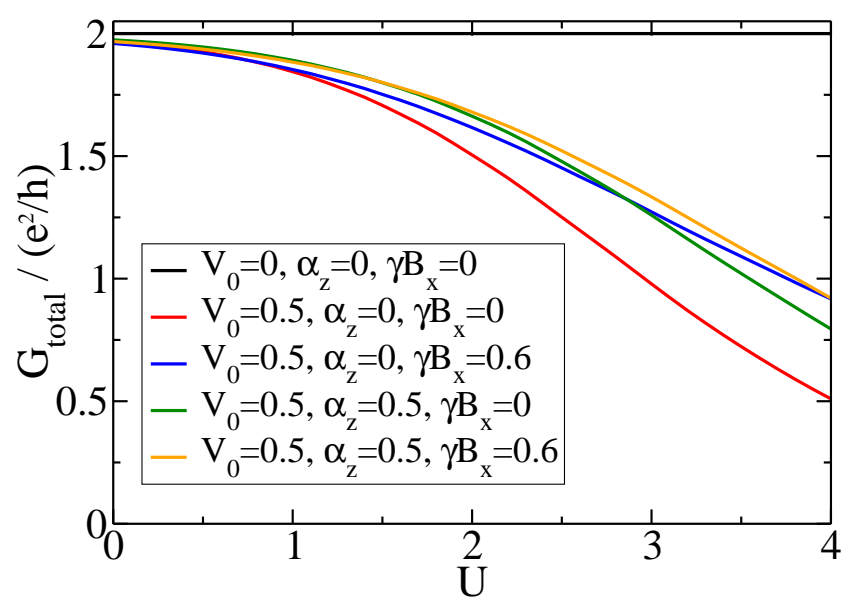

Figure 5.6: Total conductance $G_{\text {total }}$ of a system with $N=400$ lattice sites over the interaction strength $U$ at half filling for qualitatively different parameter settings. The effect that $G_{\text {total }}$ decreases with increasing $U$ in presence of a potential step $V_{0}$ is damped for finite magnetic field as well as for finite SOI.

together. Neglecting terms of order $U_{1,2}^{2} \alpha_{y, z}$ the same reasoning holds if the SOI is included.

Although we believe that the situation is far less critical for comparatively small systems of $N \sim 100$ lattice sites justifying our results mentioned above, we follow that way from now on in order to deal appropriately with somewhat larger systems as well.

For given $\mu$ and $U=U_{1}$, we choose $U_{2}=U /\left(2-\mu^{2}\right)$ in order to obtain $g_{1 \perp}=0$ [see Eq. (5.3)]. Moreover, we tune the artificial one-particle potential $\nu(U, \mu)$, which was introduced in Chapter 4 to cancel the starting value of the self energy at the beginning of the fRG flow such that the filling of the 1D quantum wire with electrons in presence of $U_{1}$ and $U_{2}$ corresponds to the filling $n$ in the non-interacting case at given $\mu$ (without $\nu$ ). According to Ref. [18, due to the integration of the flow equations from $\Lambda=\infty$ down to $\Lambda=\Lambda_{0}$ the starting values of the self energy at lattice site $j$ are given by

$$
\begin{aligned}
& \Sigma_{\sigma \sigma^{\prime}}^{\Lambda_{0}}\left(j ; j^{\prime}\right)=\left(\frac{1}{2} U_{1}(j)+2 U_{2}(j)\right) \delta_{j, j^{\prime}} \delta_{\sigma, \sigma^{\prime}}, j \in[2, N-1] \\
& \Sigma_{\sigma \sigma^{\prime}}^{\Lambda_{0}}\left(j ; j^{\prime}\right)=\left(\frac{1}{2} U_{1}(j)+U_{2}(j)\right) \delta_{j, j^{\prime}} \delta_{\sigma, \sigma^{\prime}}, j \in\{1, N\} .
\end{aligned}
$$

In order to demonstrate the influence of the Coulomb interaction, we study the total conductance as a function of the interaction strength $U=U_{1}$. Fig. [5.6 shows $G_{\text {total }}$ of a system with $N=400$ lattice sites at half filling, $\mu=0$, for qualitatively different parameter settings. As could have been expected, we obtain perfect conductance in systems without potential step or any other inhomogeneities. However, care 


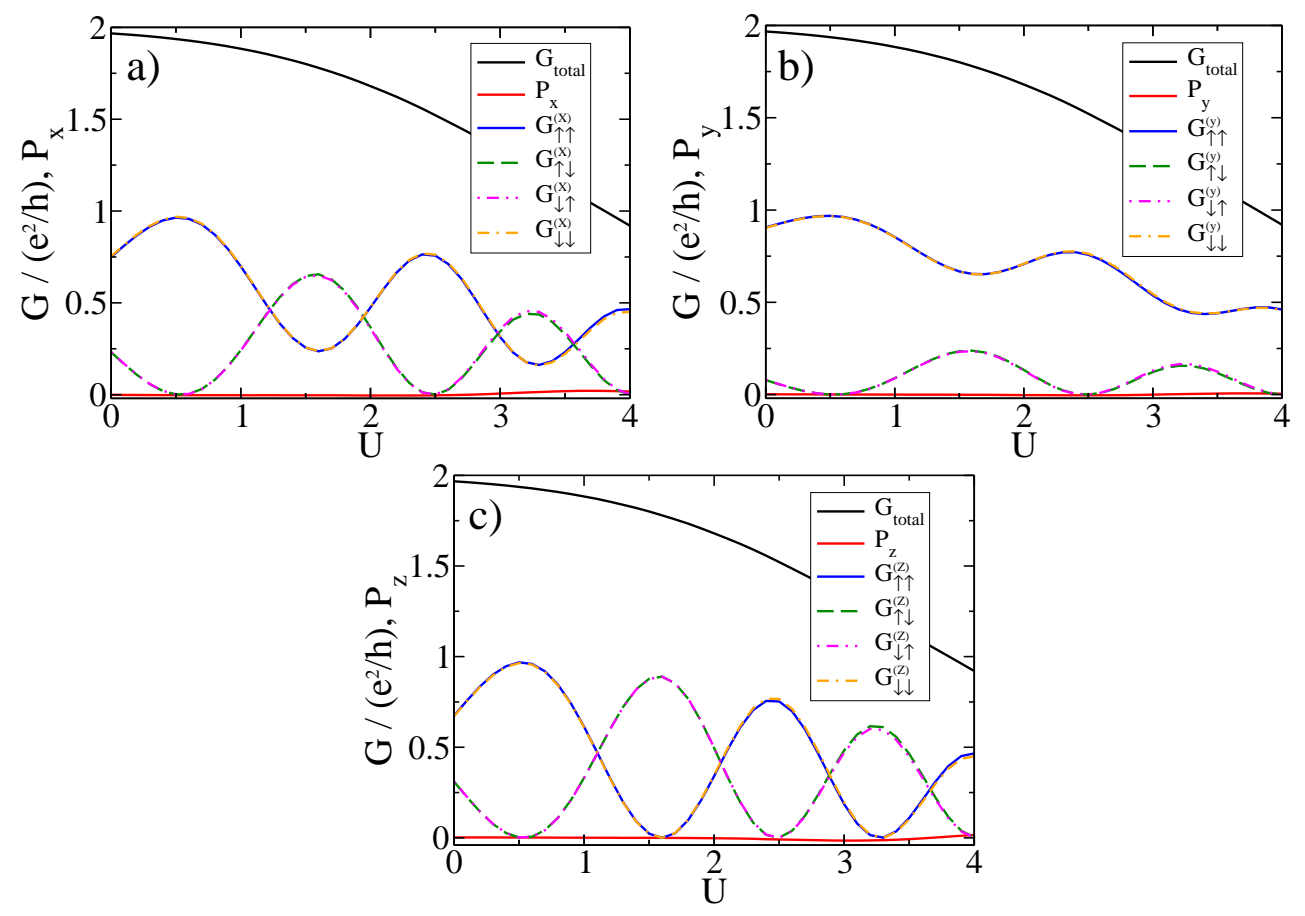

Figure 5.7: Conductance components $G_{\sigma \sigma^{\prime}}^{(x, y)}$, polarization $P_{x, y, z}$ and total conductance $G_{\text {total }}$ as a function of the interaction strength $U$ at half filling for magnetic field $\gamma B_{x}=0.6$, SOI hopping $\alpha_{z}=0.5$ and potential step $V_{0}=0.5 . G_{\sigma \sigma^{\prime}}^{(x, y, z)}$ reveal remarkable oscillations, which depend on whether the components in a) $x$ - b) $y$ - or c) $z$-direction are considered. As the spin-conserving and the spin-flip conductance components are almost degenerate, respectively, the polarization is insignificant.

must be taken for large $U$ regarding the process of adiabatically turning on/off the interaction. For $m_{1}=20$ and $U=4$, we obtain small deviations from the perfect conductance, but these deviations can be suppressed below $10^{-5}$, if we choose $m_{1}=50$, what has been done in Fig. 5.6. In presence of a potential step $V_{0}, G_{\text {total }}$ decreases with increasing $U$. Interestingly, this effect is damped in presence of a magnetic field. Independent of whether or not there is a magnetic field present in the potential step case, an additional SOI hopping $\alpha_{z}$ (or equally $\alpha_{y}$ ) further increases the conductance. Despite these modifications, the qualitative behavior of $G_{\text {total }}$ for large $U$ is governed by the potential step $V_{0}$. However, it can happen for filling $n \neq 1$ and consequently chemical potential $\mu \neq 0$ that one crosses a boundary between regimes with two and one conducting channels for increasing $U$ due to the increased renormalization of the system parameters for larger $U$.

Fig. 5.7 shows the conductance components $G_{\sigma \sigma^{\prime}}^{(i)}, i=x, y, z$, for a system with parameters $N=400, \alpha_{z}=0.5, \gamma B_{x}=0.6$ and $V_{0}=0.5$ [see orange curve in Fig. [5.6]. The conductance components $G_{\sigma \sigma^{\prime}}^{(i)}$ for spin quantization axis $i=x, y, z$ 
reveal remarkable oscillations with period $U_{\text {osc }} \sim 2$ and slowly decreasing amplitude. Since the mean value also decreases with $U$, we observe the decrease of the total conductance already shown in Fig. 5.6. The spin-conserving conductance components $G_{\sigma \sigma}^{(x, y, z)}$ and the spin-flipping components $G_{\sigma-\sigma}^{(x, y, z)}$ are almost degenerate, respectively. Thus, the polarizations $P_{x, y, z}$ are insignificant here, as could have been guessed from the fact that $\mu=0$ at half filling setting the system deep into the two conducting channel regime, where polarization has been shown to play no role. The ratio between spin-conserving and spin-flipping components strongly depends on the direction of the spin quantization axis. Since the magnetic field $B_{x}$ leads to a distinction of $\sigma=\uparrow$ and $\sigma=\downarrow$ in $x$-direction and the SOI hopping $\alpha_{z}$ drives the spin into the $y$-direction, in first order there is no difference between $\sigma=\uparrow$ and $\sigma=\downarrow$ in $z$-direction. Consequently, the oscillating spin-conserving and spin-flipping components with respect to the $z$-direction as functions of $U$ only differ by a phase $\Delta \phi=\pi$, but otherwise have the same oscillation amplitude and mean value [see Fig. 5.7 c)]. In $x$ - and $y$-direction, the phase between spin-conserving and spin-flipping components also is $\Delta \phi=\pi$, but the oscillation amplitude is reduced in comparison to the $z$-direction. Whereas it is still large enough in $x$-direction to create crossings of $G_{\sigma \sigma}^{(x)}$ and $G_{\sigma-\sigma}^{(x)}$ [see Fig. [5.7 a)], the spin-conserving components $G_{\sigma \sigma}^{(y)}$ in $y$-direction are significantly larger than $G_{\sigma-\sigma}^{(y)}$ for all $U$ [see Fig. [5.7] c)].

Further analysis shows that the ratio of $G_{\sigma \sigma}^{(y, z)}$ and $G_{\sigma-\sigma}^{(y, z)}$ depends on the (renormalized) magnetic field $B_{x}$ and the renormalization of $B_{x}$ naturally depends on the interaction strength $U$. The components $G_{\sigma \sigma}^{(x)}$ reveal no oscillations with only a magnetic field (in $x$-direction) being present, but due to the spin rotation out of the $x$-direction for finite SOI and the fact of $G_{\text {total }}$ being independent of the spin quantization axis, we obtain the oscillatory behavior also for the $G_{\sigma \sigma}^{(x)}$.

We now focus on large systems of up to $N \sim 10^{4}$ lattice sites. Such 1 D systems of correlated electrons are known to show Luttinger liquid behavior, i.e. they reveal a power law decay of correlation functions, e.g. the linear conductance. This will be explored in the following section.

\subsection{Luttinger liquid behavior in long quantum wires}

The so-called Luttinger liquids contain a large class of physical systems, e.g. interacting spinless fermions, interacting spin-1/2-fermions, Bose fluids, finite-density gas of solitons of the Sine-Gordon theory and also some kinds of spin systems. The underlying model was proposed in 1963 by J.M. Luttinger [14 and explicitely solved by Mattis and Lieb in 1965 [55]. Apparently, Luttinger was unaware of Tomonaga's work from 1950 [56], who treated a similar model, but did not realize the anomalous decay of correlation functions. However, it rather seems to be a historical fact that one does not call this class of systems Tomonaga-Luttinger liquids. A very good review on the broad spectrum of properties of Luttinger liquids can be found in Refs. [57, 58]. 


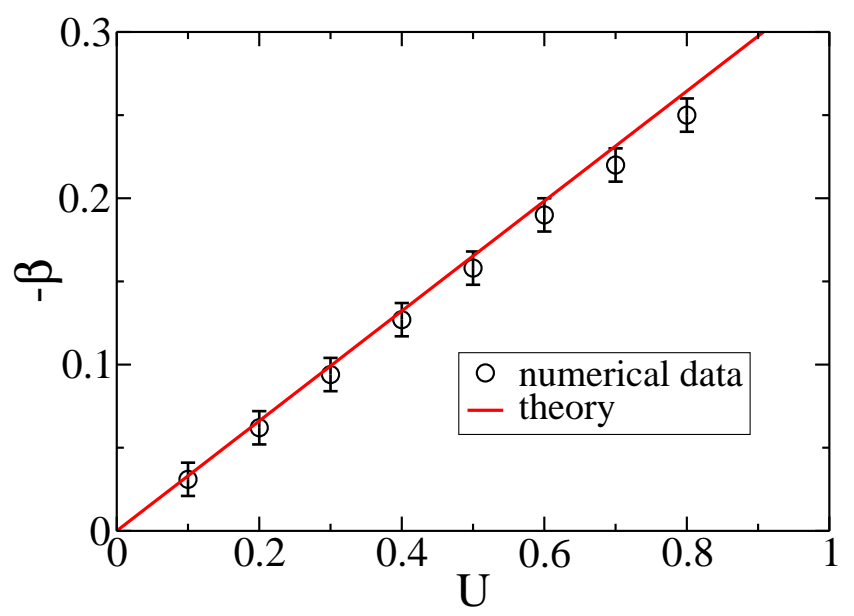

Figure 5.8: Comparison of the exponent $\beta$ obtained from the power law decay of the linear conductance for increasing system size with the theoretical value obtained from perturbation theory calculations of leading order in $U$. The filling of the system is $n=0.9$ and the asymptotical exponent is obtained for $N>2000$ lattice sites in the strong impurity regime, $V_{1}=8$.

The probably most important property of an inhomogeneous Luttinger liquid for one-dimensional transport is the power-law decay of correlation functions with temperature $T \rightarrow 0$ [59]. Since the linear conductance is defined as a current-current correlation function, this behavior should be observable in our setup as well. Instead of exploring the conductance as a function of $T$, we vary the system length $N$ the inverse of which is the relevant energy scale (up to a constant factor) of the system's low-energy excitations.

\subsubsection{Correlated electrons in systems with single impurity}

We now focus on a situation in which all system parameters like chemical potential $\mu$, Coulomb interaction $U$, SOI coupling $\alpha_{y}, \alpha_{z}$, magnetic field $B_{x}$ and single impurity $V_{1}$ are given and the system length $N$ is varied, as the relevant infrared energy scale for low-energy excitations is given by $\delta=v_{F} / N(\hbar=1)$ with $v_{F}$ being the Fermi velocity. In the absence of SOI and a magnetic field, inhomogeneities such as single impurities and potential steps, are known to lead to a power-law suppression of the linear conductance as a function of infrared energy scales [17, 18, 159, 60, 61, 62.

Our aim is to investigate how this power-law suppression is modified in presence of SOI and whether or not a similar behavior holds for the spin polarization.

Without SOI, we indeed obtain a power law decay of the conductance with exponents which correspond very well with those predicted earlier (see e.g. Refs. [59, 63]) for linear order in $U$ as shown examplarily in Fig. 5.8 for filling $n=0.9$ ( $n=1$ corresponds to half filling for spinful systems). The theoretical value of the exponent 


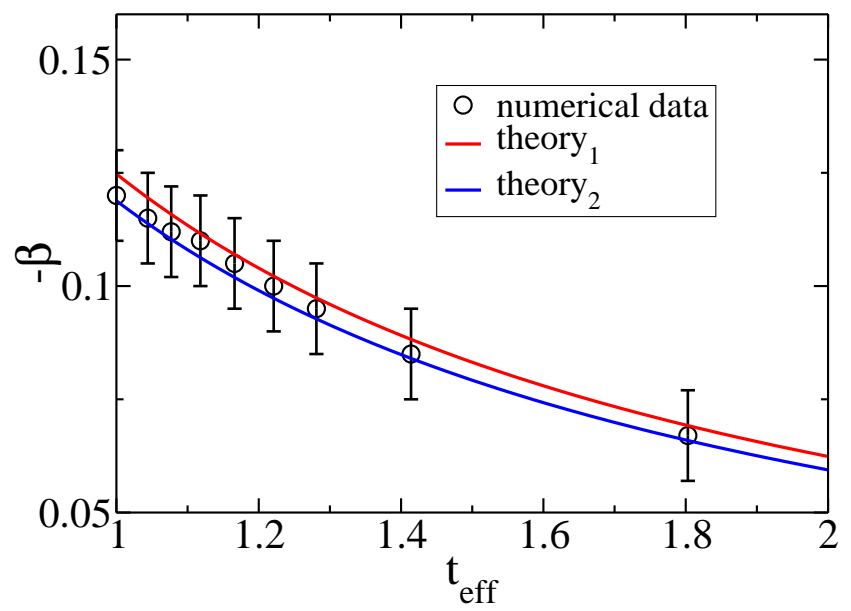

Figure 5.9: Exponent $\beta$ obtained from the power law decay of the linear conductance for increasing system size for interaction $U=0.3$, filling $n=0.75$, impurity strength $V_{1}=8$ and different SOI parameters $\alpha_{z}\left(\alpha_{y}=0\right)$ leading to different $t_{\text {eff }}$. The first theoretical curve is according to Eq. (5.5) with the hopping parameter $t$ being replaced by the unrenormalized $t_{\text {eff }}$. For the second theoretical curve, a relative increase of $5 \%$ is assumed for $t_{\text {eff }}$ due to renormalization.

$\beta$ as a function of the interaction $U$ (leading order), the filling $n$ and the chemical potential $\mu$ is given by 63 .

$$
\beta(U, n, \mu)=-\frac{\mu^{2}-4 \cos (\pi n)}{\left(2-\mu^{2}\right)(2 \pi t \sin (\pi n / 2))} U .
$$

The filling is set to $n=0.9$ by tuning $\nu(U, \mu)$, corresponding to a chemical potential $\mu=-0.313$. We choose a strong single impurity $V_{1}=8$ in the middle of the system in order to obtain the power law behavior already for systems of intermediate size. In fact, the exponent $\beta$ saturates for systems larger than $N=2000$ lattice sites.

If we now allow for finite SOI couplings $\alpha_{y}$ and $\alpha_{z}$, Eq. (5.5) still is a good approximation, but with $t$ in the denominator replaced by the effective hopping

$$
t_{\mathrm{eff}}=\sqrt{\alpha_{y}^{2}+\alpha_{z}^{2}+t^{2}} .
$$

This is shown in Fig. [5.9 for different SOI parameters $\alpha_{z}\left(\alpha_{y}=0\right)$. Again, the exponent $\beta$ has been determined from the power law decay of the linear conductance. The filling was set to $n=0.75$ and the interaction strength to $U=0.3$, i.e. $U_{1}=$ $0.3, U_{2}=0.212$. As set out in Fig. [5.8 we allow for an absolute error of \pm 0.01 in determining $\beta$, which is a rather rough estimation in this case. The data points in Fig. 5.9 are slightly below the red curve obtained from Eq. (5.5) with the hopping parameter $t$ being replaced by $t_{\text {eff }}$ according to Eq. (5.6). Note, however, that one has to insert, in theory, the renormalized hopping parameters obtained at the end 


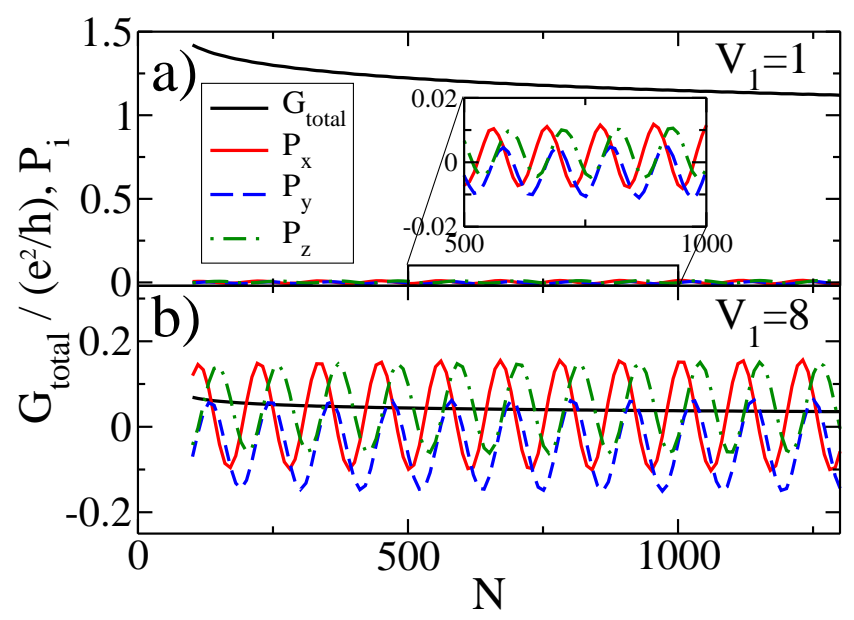

Figure 5.10: Suppression of the total conductance with increasing system size $N$ in presence of a single impurity $V_{1}$ in the middle of the system, Coulomb interaction $U=0.8$, SOI $\alpha_{y}=\alpha_{z}=0.5$ and magnetic field $\gamma B_{x}=0.6$ at $n=0.9(\mu=$ $-0.313)$. The spin polarizations show oscillations with amplitude, frequency and phase relation being independent of $N$ (for large $N$ ), both for a) small, $V_{1}=1$, and b) large, $V_{1}=8$, impurity strength.

of the fRG flow into Eqs. (5.5) and (5.6), but can also get good results with the unrenormalized parameters, since the renormalization of $\alpha_{y}, \alpha_{z}$ and $t$ is quite small as long as $U$ becomes not too large. A good measure is to assume an increase of these hopping parameters by $5-15 \%$ compared to the unrenormalized parameters. For the comparatively small interaction strength $U=0.3$ in Fig. 5.9 an increase of $t_{\text {eff }}$ by $5 \%$ (blue curve) leads to a good estimation of the lower boundary of $|\beta|$. Although the conductance (in the chosen parameter regime) is decreased with increasing magnetic field $B_{x}, \beta$ is independent of $B_{x}$.

\section{Polarization}

Another interesting question is whether or not the spin polarization shows a similar power law behavior with increasing system size. Fig. [5.10 a) shows the total conductance $G_{\text {total }}$ and spin polarization $P_{i}, i=x, y, z$, over the system size $N$ for small single impurity strength, $V_{1}=1$, at half filling $(n=1)$. The SOI couplings are $\alpha_{y}=\alpha_{z}=0.5$. In this regime, $G_{\text {total }}$ still takes quite large values and shows the typical suppression with increasing $N$, which becomes a power-law decay in the limit of large $N$. The $P_{i}$, however, show oscillatory behavior with constant amplitude and frequency for large $N$. The phase relation between the different $P_{i}$ also remains the same, independent of $N$, as can be seen from the inset in Fig. [5.10 a).

In Fig. 5.10 b), we chose $V_{1}=8$, i.e. the strong impurity regime, but otherwise used the same system parameters. One observes that the total conductance is very 


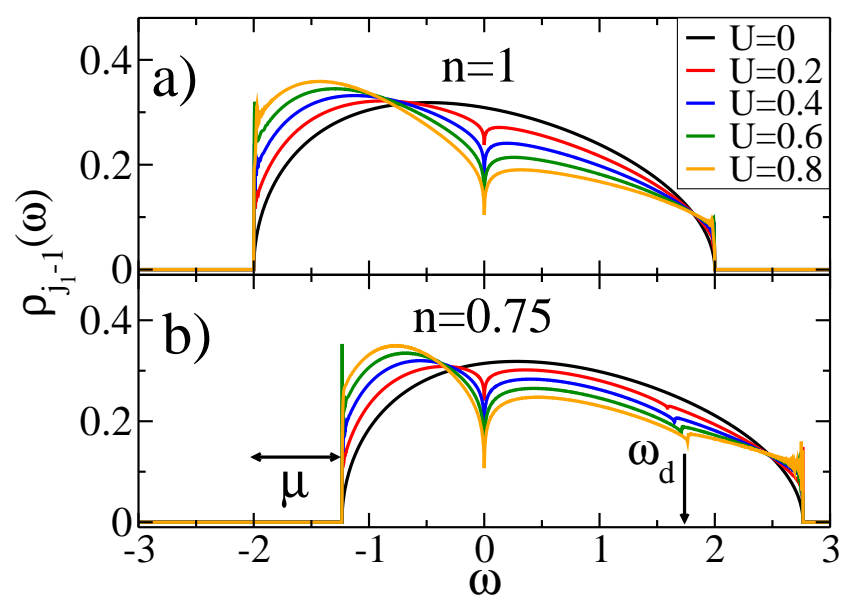

Figure 5.11: Local spectral function $\rho_{j_{1}-1}(\omega)$ next to a strong single impurity $V_{1}=8$ at site $j_{1}=[N / 2+1]$ in the middle of a system with $N=2001$ lattice sites for interaction strengths $U=0,0.2,0.4,0.6,0.8$.

a) At filling $n=1$, one observes a singularity of the spectral function at the Fermi level, i.e. at $\omega=0$.

b) For $n=0.75$, an additional suppression occurs at an interaction-dependent frequency $\omega_{d}$.

small, $G_{\text {total }}<0.1 e^{2} / h$ even for a small system and the regime of a power-law decay is already reached for an intermediate system size of $N \sim 500$. The spin polarization shows oscillations with amplitude, frequency and phase relation being independent of $N$ for smaller systems as well. Note that the polarization is increased by a factor of 10 compared to the small $V_{1}$ regime while exhibiting the same frequency and phase relation. All these features remain valid, if the system size is increased any further.

Thus, although the spin polarization does not depend on the system size, apart from the oscillatory behavior, one would obtain a reduced spin current for larger systems due to the Luttinger liquid behavior, i.e. the power law decay of the total conductance.

In the following, we show that the exponent of the power law extracted from the local spectral function next to the impurity corresponds to the result obtained from the decay of the linear conductance.

\section{Spectral function}

Another approach to determine the exponent $\beta$ is to consider the local spectral function $\rho_{j}(\omega)$ defined by

$$
\rho_{j}(\omega)=-\frac{1}{\pi} \Im\left(\mathcal{G}_{j, j}^{\Lambda=0}(\omega+i 0)\right)=-\frac{1}{\pi} \Im\left(\left[\omega+i 0+\mathcal{G}_{0}^{-1}-\Sigma^{\Lambda=0}\right]_{j, j}^{-1}\right)
$$


with some real frequency $\omega$. Having integrated the flow equations for the self energy $\Sigma^{\Lambda}$ down to $\Lambda=0$, we obtain an approximation to the physical self energy $\Sigma^{\Lambda=0}$ and the single-particle propagator $\mathcal{G}^{\Lambda=0}$ from which all properties of single-particle excitations can be extracted. Fig. [5.11 shows the local spectral function $\rho_{j_{1}-1}(\omega)$ next to a strong single impurity $V_{1}=8$ at site $j_{1}=[N / 2+1]$ in the middle of a system with $N=2001$ lattice sites. Note that the exact position of the impurity is irrelevant as long as it is far away from the lead contacts. At half filling, $n=1$ [see Fig. $5.11 \mathrm{a})$ ], one observes finite values of $\rho_{j_{1}-1}(\omega)$ for $\omega$ being within the energy band, i.e. $\omega \in(-2,2)$ (in units of the lead hopping). Besides the vanishing towards the band edges one observes a dip at the Fermi energy $\omega=0$, which is the more pronounced the larger $U$. This certainly is a correlation effect, as it does not occur in the non-interacting case. For $n=0.75$ [see Fig. [5.11] b)], the overall chemical potential is $\mu=-0.765$ and one observes finite values of the local spectral function for $\omega \in(-2+\mu, 2+\mu)$. In addition to the dip at $\omega_{1}=0$, one finds a slight dip at an interaction-dependent frequency $\omega_{d}$. As pointed out in Ref. 64, this effect comes from the fact that the narrow potential $V_{1}$ in lattice space is a longe-range potential in $k$-space with interaction-induced Friedel oscillations of frequency $2 k_{F}$. Thus, not only states with momenta close to $k_{F}=\pi n / 2$ are scattered at this potential, but also states with momenta close to $\pi-k_{F}$. Essentially, the second dip is expected to be located at $\omega_{2}=\tilde{\epsilon}\left(\pi-k_{F}\right)-\mu$ with

$$
\tilde{\epsilon}(k)=-2 \tilde{t}_{\text {eff }} \cos (k)+\tilde{V}
$$

being the renormalized dispersion. $\tilde{t}_{\text {eff }}$ is the renormalized effective hopping and the additional one-particle potential $\tilde{V}$ being created within the fRG flow accounts for the fact that the chemical potential $\mu=\tilde{\epsilon}\left(k_{F}\right)$ is constant in the entire setup (system and leads). This is assured by the careful tuning of $\nu$. From Fig. $5.11 \mathrm{~b}$ ), one obtains the position of the second $\operatorname{dip} \omega_{d}$. The following table shows a very good correspondence to the theoretical value $\omega_{2}$. The values of $\tilde{V}$ and $\tilde{t}_{\text {eff }}$ obtained from the self energy $\Sigma^{\Lambda=0}$ at the end of the fRG flow are also shown for convenience.

\begin{tabular}{c|c||c|c|c|c|}
$n=0.75$ & $U$ & $\omega_{d}$ & $\omega_{2}$ & $\tilde{t}_{\text {eff }}$ & $\tilde{V}$ \\
\hline & 0.2 & 1.591 & 1.593 & 1.041 & 0.031 \\
& 0.4 & 1.651 & 1.655 & 1.081 & 0.062 \\
& 0.6 & 1.710 & 1.713 & 1.119 & 0.091 \\
& 0.8 & 1.764 & 1.770 & 1.156 & 0.119
\end{tabular}

The relation $\omega_{d}=\omega_{2}$ should hold for all fillings $n$. Indeed, we observe an analogue correspondence for $n=0.9$.

\begin{tabular}{c|c||c|c|c|c|}
$n=0.9$ & $U$ & $\omega_{d}$ & $\omega_{2}$ & $\tilde{t}_{\text {eff }}$ & $\tilde{V}$ \\
\hline & 0.2 & 0.645 & 0.646 & 1.033 & 0.010 \\
& 0.4 & 0.665 & 0.666 & 1.065 & 0.020 \\
& 0.6 & 0.685 & 0.686 & 1.097 & 0.030 \\
& 0.8 & 0.705 & 0.706 & 1.129 & 0.040
\end{tabular}


A comparison of the two tables also shows that the renormalization of the additional one-particle potential $V$ and the effective hopping $t_{\text {eff }}$ is increased, if one goes further away from half filling.

It is a pecularity of half filling, $n=1$, that there is only one dip in the spectral function at $\omega_{1}=0$, since $k_{F}=\pi n / 2=\pi-k_{F}$ and therefore $\omega_{1}=\omega_{2}$ [see Fig. 5.11] a)].

Coming back to our original purpose, namely the identification of the exponent $\beta$ from the spectral function, there are two possible ways. A thorough investigation shows that $\rho_{j_{1}-1}$ can be fitted very well on either side of $\omega= \pm 0$ by a power law with exponent $\beta_{L}^{+}$for $\omega \rightarrow 0+$ and $\beta_{L}^{-}$for $\omega \rightarrow 0$ - with the natural symmetry $\beta_{L}^{+}=\beta_{L}^{-}$. Similarly, we obtain $\beta_{R}^{+}=\beta_{R}^{-}$for the exponents from fitting $\rho_{j_{1}+1}$ around $\omega= \pm 0$. It will be shown in Sect. 5.2.2 that the exponents on the left/right side of the single impurity can be different in presence a potential step. However, with just a single impurity present, we obtain a degeneracy of all four exponents. Taking the sum of these exponents, we obtain exactly the exponent $\beta_{G}$ which was determined from the decay of the linear conductance with increasing system size up to a numerical deviation of $\mathcal{O}\left(10^{-3}\right)$.

Another possibility is to examine the values of $\rho_{j_{1}-1}(\omega=0)$ and $\rho_{j_{1}+1}(\omega=0)$ as a function of system size $N$. Again, one obtains power laws with exponents $\beta_{L}(\omega=0)$ for $\rho_{j_{1}-1}(\omega=0)$ and $\beta_{R}(\omega=0)$ for $\rho_{j_{1}+1}(\omega=0)$, which exactly match the corresponding sums

$$
\begin{aligned}
& \beta_{L}(\omega=0)=\left(\beta_{L}^{+}+\beta_{L}^{-}\right) / 2 \\
& \beta_{R}(\omega=0)=\left(\beta_{R}^{+}+\beta_{R}^{-}\right) / 2
\end{aligned}
$$

and therefore

$$
\beta_{G}=\beta_{L}(\omega=0)+\beta_{R}(\omega=0)
$$

with $\beta_{G}$ being the exponent extracted from the power law decay of the total conductance.

\subsubsection{Correlated electrons in systems with potential step}

We now analyze whether a power law behavior of the linear conductance can also be observed in presence of a potential step $V_{0}$. It was shown above that one needs to implement quite large single impurities, $V_{1} \sim 8$, into the system in order to observe a well-pronounced power law decay of the linear conductance already for an intermediate system size $N \sim 10^{3}$.

Although the simple one-particle picture described in Fig. 3.8 does not hold in presence of Coulomb interaction, one could intuitively guess that the conductance will be zero, if $V_{0}$ is chosen too large, as there will be no propagating states on the left side of the potential step with energies matching the energies of propagating states of the right side (trivial band effect). Such behavior can indeed be observed, even for comparatively small systems with $N \sim 100$ lattice sites. 


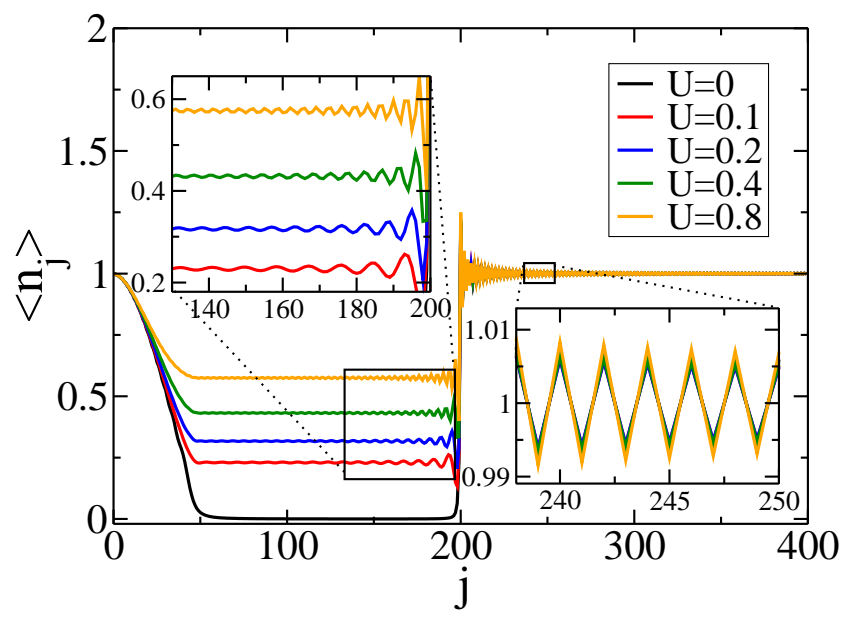

Figure 5.12: Average particle number $\left\langle n_{j}\right\rangle$ over lattice site $j$ for a system of size $N=400$ with a potential step of height $V_{0}=2$ in the middle of the system. $V_{0}$ is turned on adiabatically over $m_{1}=50$ lattice sites and extends over the left part of the system. $\left\langle n_{j}\right\rangle$ strongly depends on $U$ on the left side, but is independent from $U$ on the right side. One observes typical Friedel oscillations with frequency corresponding to the particular filling.

\section{Particle density}

Fig. 5.12 shows the profile of the particle density $\left\langle n_{j}\right\rangle=\left\langle n_{j, \uparrow}\right\rangle+\left\langle n_{j, \downarrow}\right\rangle$ for a system of size $N=400$ at $\mu=0$ ( $n=1$, half filling) for different interaction strengths $U$ without SOI and magnetic field.

Note that the case $n=1$ has one peculiarity. It has been shown for the Hubbard model with vanishing SOI that the system at half filling is driven towards a Mott insulator phase for large $N$ in presence of impurities [65]. However, this phase transition is not captured within our fRG approach and the results are continuous with respect to $n$. Therefore, our results obtained for $n=1$ are to be understood in this continous limit.

Again, the interaction has been tuned such that $U_{1}=U$ and $U_{2}=U_{1} / 2$ in order to achieve $g_{1 \perp}=0$. A potential step of height $V_{0}=2$ is included in the middle of the system. The potential is turned on adiabatically and extends over the left part of the system. As mentioned in the previous section, we also included the one-particle potential $\nu(U, \mu=0)$ that ensures the correct filling $n=1$ (for $\mu=0$ ) for the homogeneous system. One observes impurity-induced density fluctuations, known as Friedel oscillations, which start at the potential step with quite a large amplitude and decay towards the boundaries of the system. As pointed out in Sect. 4.3. our definition of $\left\langle n_{j, \sigma}\right\rangle$ according to Eq. (4.31) is just an approximation which does not account for the correct power-law decay of the Friedel oscillations' amplitude as a function of distance from the impurity. However, the average particle 

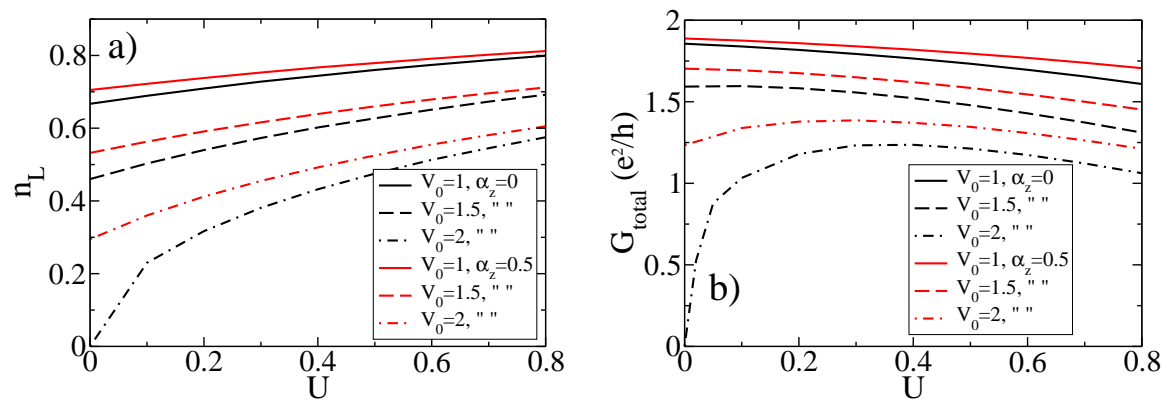

Figure 5.13: a) Filling $n_{L}$ on the left side of the system under consideration in Fig. 5.12 for different heights $V_{0}$ of the potential step. In presence of SOI, $\alpha_{z}=0.5$, $n_{L}$ is increased especially for small interaction strength $U$.

b) The linear conductance $G_{\text {total }}$ is increased for finite $\alpha_{z}=0.5$ for all $U$ and $V_{0}$. The vanishing of $G_{\text {total }}$ for $V_{0}=2$ and $U \rightarrow 0$ corresponds to the behavior of $n_{L}$ in this parameter regime.

number (the filling) and the frequency of the Friedel oscillations are correct within our approximation 43 .

As can be seen from the right inset in Fig. 5.12 , the particle density on the right side oscillates around $n=1$ with period $j_{\text {osc }}=2$, independent of $U$. In contrast, the particle density on the left side is almost zero for the non-interacting case (in fact, $n \sim 10^{-3}$ ) and is strongly increased with $U$. The non-interacting case corresponds very well to the picture described by Fig. 3.8 Since we chose $V_{0}=2$, the left dispersion is just shifted by $\epsilon=2$ matching $\mu=0$ only at the very bottom of its energy branches which are degenerate, because there is no SOI and no magnetic field. Therefore, one can expect that these parameters just indicate the boundary between zero and finite transmission. This behavior is also reflected in the (almost) vanishing particle density. The most astonishing feature of the left inset in Fig. [5.12 is that $\left\langle n_{j}\right\rangle$ is severely increased with $U$. A thorough investigation shows that the frequency of the corresponding oscillation corresponds very well to the Fermi wave vector $k_{F}=\pi n / 2$ such that the particle profile in the bulk of the left part can be modelled for each interaction by a superposition of the corresponding filling $n_{L}$ (average particle number on the left side) and the Friedel oscillations via

$$
n_{j}=n+A(j) \sin (\pi n j+\Phi)
$$

with all parameters depending on $U . A(j)$ accounts for the decaying amplitude of the oscillations and $\Phi$ is an additional phase shift with no physical meaning. The filling $n_{L}$ of the left part of the system is defined by the arithmetic mean of $n_{j}$ for $j$ being in the left bulk, i.e.

$$
n_{L}=\frac{1}{N / 2-m_{1}} \sum_{j>m_{1}}^{N / 2} n_{j},
$$




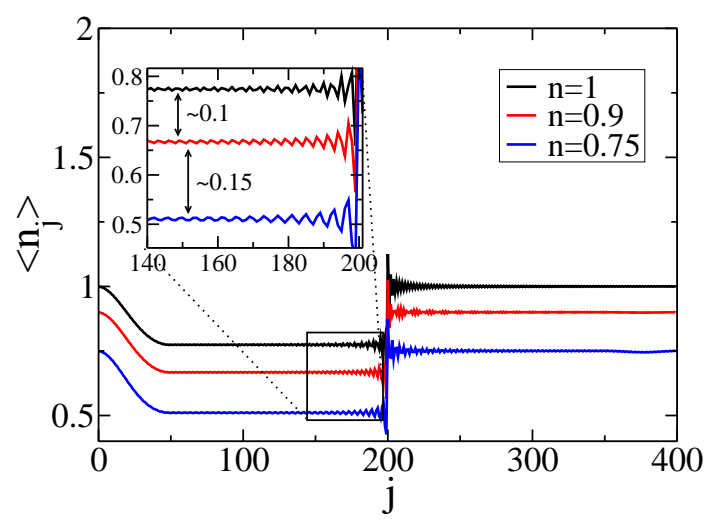

Figure 5.14: Profile of the particle number $\left\langle n_{j}\right\rangle$ for constant interaction strength $U=0.6$, potential step $V_{0}=1$ and fillings $n=1,0.9,0.75$. The average particle number on the right side corresponds very well to the predetermined filling. The average particle number on the left side is decreased by a certain amount independent from $n$, as shown in the inset.

and is analyzed in Fig. $5.13 \mathrm{a}$ ). Naturally, $n_{L}$ decreases with increasing $V_{0}$, whereas the $U$-dependence of $n_{L}$ strongly increases for $V_{0} \rightarrow 2$, which was shown to define the crossover between zero and finite conductance in the non-interacting case. Assuming finite SOI, $\alpha_{z}=0.5$, leads to larger $n_{L}$, especially for small interaction strength $U$, but the asymptotic value of $n_{L}$ which seems to exist for very large $U$ does not seem to depend significantly on $\alpha_{z}$. One should note that the influence of $\alpha_{z}$ (or accordingly $\alpha_{y}$ ) can again be absorbed into an effective hopping $t_{\text {eff }}=\sqrt{t^{2}+\alpha_{y}^{2}+\alpha_{z}^{2}}$.

Fig. $5.13 \mathrm{~b}$ ) shows the linear conductance $G_{\text {total }}$ for the corresponding parameters. $G_{\text {total }}$ is increased for all $V_{0}$ and $U$ in presence of SOI, $\alpha_{z}=0.5$, in correspondence to the increased filling $n_{L}$. Taking the potential step into account as an impurity, one would expect that $G_{\text {total }}$ scales down with increasing $U$. For $V_{0}=2$ however, one observes an increase of $G_{\text {total }}$ for small $U$ up to a maximum value and a subsequent decrease for larger $U$. This behavior has no direct analogue to the filling $n_{L}$, but at least the suppression $G_{\text {total }} \rightarrow 0$ for $U \rightarrow 0$ is connected to the disappearance of the particles on the left side.

Going away from half-filling, we observe for the case with $U=0.6, \alpha_{z}=0$ and otherwise the same system parameters as above that again the filling $n_{R}$ on the right side of the system is the predetermined filling set by the chemical potential $\mu$ of the leads and the interacting region [see Fig. [5.14]. The small dent in the $n=0.75$ curve on the right system boundary is just a numerical peculiarity, since $U_{1}$ and $U_{2}$ need to be switched on/off with the same function entering the initial values of the self energy, but need to be switched on/off with different functions in the fRG flow equation accounting for the fact that $U_{1}$ is an on-site and $U_{2}$ a nearest-neighbor property of the system, but this has no physical relevance. Furthermore, one should 

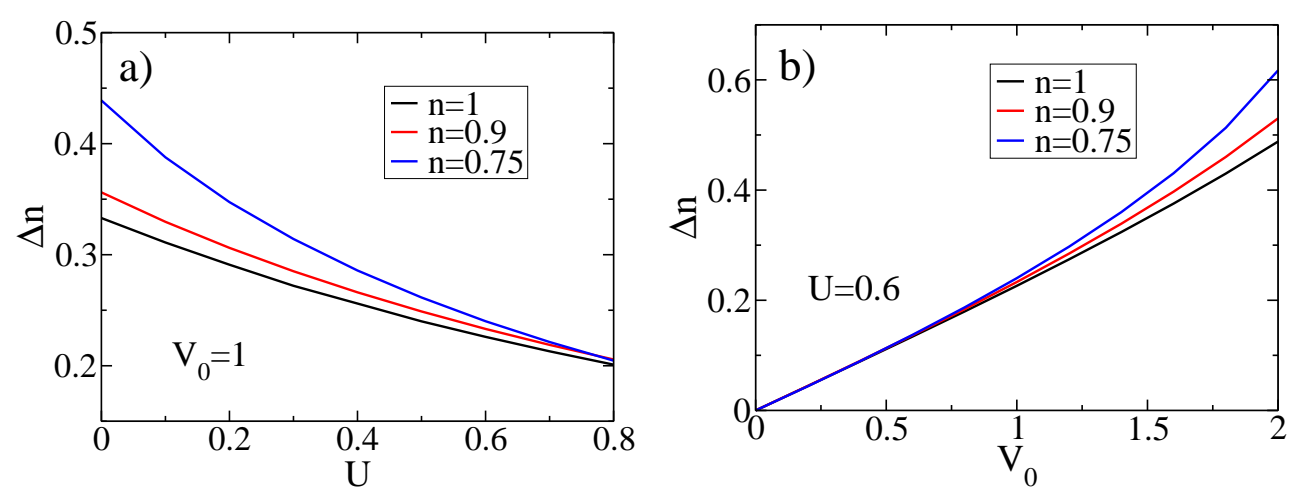

Figure 5.15: Difference $\Delta n$ between the filling on the right side and the left side of the potential step at the end of the fRG flow for initial filling $n=1,0.9,0.75$.

a) For constant $V_{0}=1, \Delta n$ becomes independent of the initial filling $n$, if $U$ is increased.

b) For constant $U=0.6, \Delta n$ is independent of $n$ and grows linearly for small $V_{0}$, but splits up for $V_{0}>0.5$.

note that due to the condition $g_{1, \perp}=0$, i.e. $U=U_{1}=U_{2}\left(2-\mu^{2}\right), U_{2}$ depends on $n$ for constant $U$, since $\mu$ depends on $n$. However, a direct comparison of the curves with the same $U$ but different $n$ should give, at least qualitatively, a rough understanding of the underlying physics.

The difference $\Delta_{n}=n_{R}-n_{L}$ between the fillings on the right side and the left side does not seem to depend significantly on $n$, as should be clear from the inset of Fig. 5.14 since the difference between the fillings $n_{L}$ on the left side is the difference between the fillings $n_{R}$ on the right in good approximation.

However, a more thorough investigation shows that this is not the case for the entire parameter regime. Fig. [5.15] a) clarifies that $\Delta n$ decreases for all fillings $n=1,0.9,0.75$ as a function of $U$ for constant potential step height $V_{0}=1$. Starting from a significant dependence on $n$ for the non-interacting case, the different curves converge for larger $U$. For constant $U=0.6$, Fig. [5.15] b) shows that $\Delta n$ is degenerate with respect to $n$ and grows linear for small $V_{0}$. For $V_{0}>0.5$, the $n$-degeneracy is lifted and the increase of $\Delta n$ is enhanced the more the smaller $n$. Thus, one can expect a competitive behavior of $V_{0}$ and $U$ in affecting $\Delta n$.

Although the filling $n_{L}$ on the left side of the potential step, i.e. the area where there actually is a one-particle potential $V_{0}$, strongly depends on the system parameters, the filling $n_{R}$ on the right side seems to be independent of any parameters but the predetermined filling $n$. This clearly conflicts with Ref. [66] which refers to a slightly different situation. The setup under consideration there was a system of interacting electrons with impurity in the middle of the system and two different Fermi wave vectors $k_{F}=k_{L, R}$ on the left/right side of the scatterer. In a way, this corresponds to our setup with the one-particle potential $V_{0}$ on the left side and the 


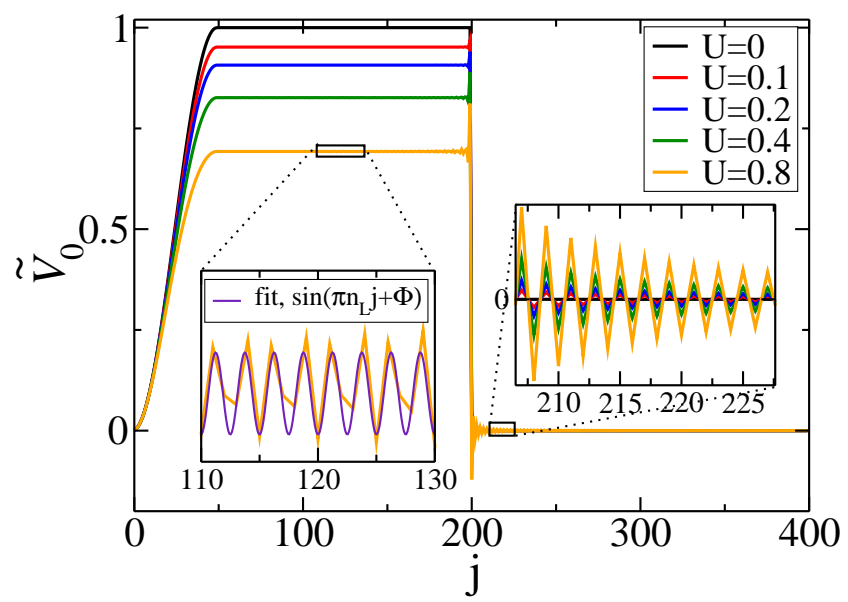

Figure 5.16: Renormalized potential $\tilde{V}_{0}$ over the lattice index $j$ for a system with $N=400$ lattice sites and interaction strength $U=0,0.1,0.2,0.4,0.8$ at half filling, $n=1$. The adiabatic turning-on of the potential over $m_{1}=50$ lattice sites and the potential step in the middle of the system can clearly be observed. The interaction $U$ leads to a decrease of $\tilde{V}_{0}$ in the left region, but does not induce a potential in the right region. The typical Friedel oscillations can be seen on both sides with frequencies corresponding to the respective fillings.

potential step in the middle of the system leading to different fillings $n_{L, R}$ and therefore different Fermi wave vectors $k_{F}=k_{L, R}$ on both sides. However, no leads were included in the model studied in Ref. [6] and the authors assumed two connected semi-infinite systems with $k_{F}=k_{L}$ and $k_{F}=k_{R}$, respectively. For this setup, one observes two different frequencies $\omega_{L, R}$ of Friedel oscillations corresponding to the particular $k_{L, R}$ for the non-interacting case. For finite interaction, one should observe an additional peak in the spectrum of density oscillations at the counterpart $k_{R, L}$ leading to a beating pattern in the Friedel oscillations, i.e. a superposition of two density oscillations with frequency $\omega_{L, R}$ 66. However, we do not observe such behavior in our setup with a finite system connected to the non-interacting leads.

In order to get a deeper insight into the observed phenomena, we now focus on the renormalized potential $\tilde{V}_{0}$ which can be calculated from the diagonal elements $\Sigma_{j, j}^{\Lambda=0}(\sigma, \sigma)$ of the self energy at the end of the fRG flow. Fig. [5.16] shows the profile of the renormalized potential $\tilde{V}_{0}$ for a system with $N=400$ lattice sites and interaction srength $U=0,0.1,0.2,0.4,0.8$. The unrenormalized potential $V_{0}$ entering the initial condition of the system's self energy again is turned on adiabatically over $m_{1}=50$ lattice sites and abruptly turned off in the middle of the system leading to a potential step of height $V_{0}=1$. Besides the Friedel oscillations, no potential is generated in the right area. This explains why no change in the filling $n_{R}$ on the system's right side was observed in Figs. 5.12 and 5.14. According to Eq. (5.11), the frequency of the oscillation is determined by the particular filling. $n_{R}=1$ on the right side of the 


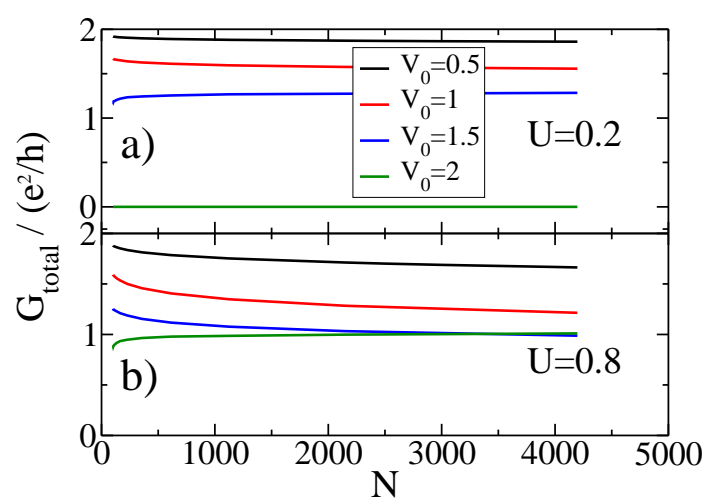

Figure 5.17: Total conductance $G_{\text {total }}$ over system size $N$ for $n=0.75$ and potential step height $V_{0}=0.5,1,1.5,2$.

a) For $U=0.2, G_{\text {total }}$ vanishes for $V_{0}=2$ independent from the system size. The conductance for smaller potential steps obtains sizeable values and depends only weakly on the system size.

b) For $U=0.8$, the $V_{0}=2$-conductance is finite and reveals a slight increase with $N$. $G_{\text {total }}$ decreases for smaller $V_{0}$ with increasing $N$.

system leads to $\omega_{R}=\pi n_{R}=\pi$ as can be seen from the right inset in Fig. 5.16. The left filling is $n_{L}=0.693$ and the corresponding frequency $\omega_{L}=\pi n_{L}$ used for the fit in the left inset is a fairly good approximation to the oscillations of $\tilde{V}_{0}$ (naturally, one could not expect a perfect correspondence, as the lattice space is discrete). Thus, we obtain a universal frequency of the Friedel oscillations holding not only for the filling but also for the renormalized potential.

\section{Conductance}

For the single impurity case, we have shown in Sect. 5.2.1 that only in the strong impurity regime $\left(V_{1} \sim 8\right)$ a well-defined power law decay of the conductance with increasing system size can be observed. Thus, it is questionable whether we can obtain such behavior in systems with potential step $V_{0}$, in which one is much more limited in choosing the height of $V_{0}$ in order to still obtain a finite conductance. Indeed, one has seen in Fig. 5.13 that a certain $V_{0}$ exists which defines a crossover value leading to a severely suppressed total conductance $G_{\text {total }}$ for small interaction strength $U$. In presence of a single impurity, one observed a decrease of $G_{\text {total }}$ with increasing system size $N$. However, Fig. 5.17 shows that in presence of a potential step $V_{0}$ the opposite behavior can be observed as well. For e.g. $U=0.2$ and $V_{0}=1.5$, the total conductance increases with growing system size [see Fig. [5.17 a)]. The same behavior is observed in Fig. $5.17 \mathrm{~b}$ ) for $U=0.8$ and $V_{0}=2$. However, the most interesting feature is the fact that one obtains a quite sizeable conductance $G_{\text {total }} \sim 1 e^{2} / h$ for $V_{0}=2$ and $U=0.8$, whereas it vanishes for $V_{0}=2$ and $U=0.2$. This fact fits very well to the picture mentioned above for the vanishing particle 
densities. Whereas we observed finite particle densities in the left half of the system in Fig. 5.12 for $V_{0}=2$ and $U=0.2$ at $n=1$, i.e. $\mu=0$, the particle density in the left half vanishes for the same parameters at $n=0.75$, i.e. $\mu=-0.765$, establishing once more the idea set out in Fig. 3.8.

Furthermore, all curves in Fig. 5.17 have in common that any fit according to a power law suffers from large discrepancy to the data, as the $2 k_{F}$ scattering component $\tilde{V}_{0}\left(2 k_{F}\right)$ of the renormalized Fourier-transformed potential is not large enough in order to drive the system into the power-law regime. Choosing larger $V_{0}$ would lead to a total suppression of $G_{\text {total }}$ due to the trivial band effects.

Thus, we implement a strong single impurity $V_{1}=8$ in the middle of the system in addition to the potential step in order to force the system into the parameter regime in which a power law behavior can be observed. Indeed, we achieve a decay of the linear conductance with increasing system size, which can be fitted well by a power law, at least for comparatively small height of the potential step, i.e. $V_{0}<1$.

Since we have shown above that a potential step leads to two subsystems with different fillings $n_{L, R}$, one could expect that one can derive the exponents $\beta_{L, R}^{+/-}$and $\beta_{L, R}(\omega=0)$ from the spectral function [see Sect. [5.2.1], which correspond to the particular filling on the left/right side of the potential step according to Eq. (5.5). However, we will see that this is not the case in the present setup.

In the same manner as for the single impurity case in Sect. 5.2.1, we observe the relationship

$$
\begin{aligned}
& \beta_{L}(\omega=0)=\left(\beta_{L}^{+}+\beta_{L}^{-}\right) / 2 \\
& \beta_{R}(\omega=0)=\left(\beta_{R}^{+}+\beta_{R}^{-}\right) / 2
\end{aligned}
$$

and

$$
\beta_{G}=\beta_{L}(\omega=0)+\beta_{R}(\omega=0) .
$$

As expected, the exponents $\beta_{R}(\omega=0)$ and $\beta_{R}^{+/-}$taken from the spectral function $\rho_{j_{1}+1}$ on the right side do not depend on the height $V_{0}$ of the potential step. This corresponds to the fact that the filling $n_{R}$ on this side of the system is also independent from $V_{0}$. The right exponents therefore correspond very well to the theoretical prediction [see Eq. (5.5)]. As the minimum of Eq. (5.5) is at half filling for all interactions $U$, one would expect an increase of the left exponents $\beta_{L}(\omega=0)$ and $\beta_{L}^{+/-}$ for finite $V_{0}$, since $n_{L}$ departs from $n=1$ in this case. Fig. 5.18 shows the comparison of $\beta_{L}(\omega=0)$ as a function of $U$ for different potential steps, $V_{0}=0,0.5,0.8$. The original filling was set to $n=1$, which equals $n_{L}$ for $V_{0}=0$. Increasing $V_{0}$ at constant $U$ leads to a reduced $n_{L}$. The theoretical values have been obtained from Eq. (5.5) with $n$ being replaced by $n_{L}$. Although still being increased with $U$ the slope of the data shows the opposite trend than would be expected from the theory.

This behavior can not be explained in our framework. We emphasize that the tuning $g_{1, \perp}=0$ does not hold in this setup, since the filling on the left side does not correspond to the chemical potential. The quality of our data might suffer from this fact. However, our data show an unexpected trend even for the situation of very 


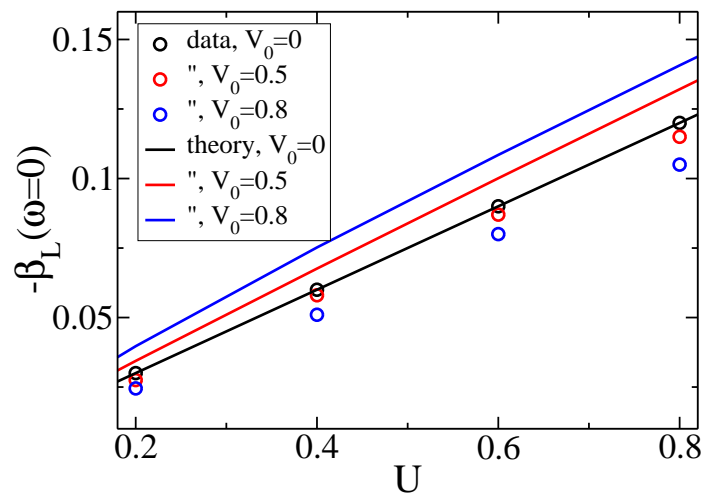

Figure 5.18: Comparison of the exponent $\beta_{L}(\omega=0)$ for a system with filling $n=1$, single impurity $V_{1}=8$ and potential step $V_{0}=0,0.5,0.8$ with the theoretical value, which follow from Eq. (5.5) for the corresponding filling $n_{L}$ on the left side of the system. The data shows the opposite trend as would be expected from the theory.

small $U$ in which $g_{1, \perp} \neq 0$ might not be that crucial. This indicates that the idea of two individual subsystems with filling $n_{L, R}$ on the left/right side of the potential step inducing two different exponents $\beta_{L, R}(\omega=0)$ should be considered with caution.

\subsection{Summary}

The repulsive Coulomb interaction has been modelled by an on-site part $U_{1}$ and a nearest-neighbor part $U_{2}$ in analogy to the extended Hubbard model. It has been shown that sharply turned on/off system parameters which are not present in the leads result in severe conductance oscillations for finite $U_{1}$ and $U_{2}$. Therefore, we chose a smooth weight function to turn on/off the Coulomb interaction, SOI and magnetic field over a certain number $m_{1}$ of lattice sites. $m_{1} \sim 20$ emerged to be sufficient for short wires $(N \lesssim 400)$ and in order to suppress these oscillations and to obtain a perfect conductance over the entire bandwidth. For long wires of up to $N=10^{4}$ lattice sites, we got perfect conductance for $m_{1} \sim 50$.

Analyzing the conductance as function of the chemical potential, we observed a modification of the areas with zero, one and two conducting channels, which has been attributed to the renormalization of the magnetic field and the SOI parameters. This resulted also in a change of the parameter range where spin polarization is achieved.

Since we omitted the flow of the two-particle vertex in our approach, we chose a certain $\mu$-dependent ratio $U_{1} / U_{2}=-2 \cos \left(2 k_{F}\right)=2-\mu^{2}$ in order to tune the $2 k_{F}$-backscattering component $g_{1, \perp}$ to zero and to guarantee the correctness of our results for larger systems of up to $N \sim 10^{4}$ lattice sites as well.

The conductance as function of the interaction strength $U$ at half filling was shown to severely decrease in presence of even small potential steps $V_{0}$. However, this decrease could be damped for finite magnetic field or finite SOI hopping. How- 
ever, the qualitative conductance behavior for large $U$ depends mainly on $V_{0}$. The conductance components plotted over $U$ revealed pronounced oscillations the amplitude of which depending on the direction of the spin quantization axis. The origin of these oscillations could be traced back to the subtle interplay of SOI and magnetic field both of which are effectively changed for finite Coulomb interaction due to renormalization.

Luttinger liquid behavior in form of a power law decay of the conductance with increasing system size could be observed in presence of a strong single impurity $V_{1}$ in the middle of the system. The power law exponent $\beta$ was shown to fit very well to the theoretical predictions for linear order in $U$. The influence of SOI could be absorbed into an effective hopping $t_{\text {eff }}$ and led to an exponent $\beta \sim 1 / t_{\text {eff }}$. For finite Coulomb interaction, we observed two dips in the local spectral function $\rho_{j_{1} \pm 1}(\omega)$ on the lattice site $j_{1} \pm 1$ next to the single impurity. The dips indicated the scattering of states with momentum $k_{F}$ and $\pi-k_{F}$ off the single impurity which, in $k$-space, could be seen as a long-range potential with interaction-induced Friedel oscillations of frequency $2 k_{F}$. The decay of $\rho_{j_{1} \pm 1}(\omega)$ for $\omega \rightarrow 0 \pm$ and the decay of $\rho_{j_{1} \pm 1}(\omega=0)$ for increasing system size led to the same exponent as the one being extracted from the conductance.

Thereafter, we studied systems of correlated electrons with a broad potential $V_{0}$ which was turned on adiabatically in the left part of the system and turned off sharply in the middle of the system leading to a potential step. The filling $n_{R}$ on the right system side shaped up as being independent of the interaction strength and the height of the potential step and matching exactly the filling $n$ which was predetermined by the overall chemical potential, whereas the filling $n_{L}$ on the left side revealed a strong dependence on the system parameters and eventually (almost) vanished for large $V_{0}$. This could be explained, at least qualitatively, by the energetical rupture of the system in a one-particle picture with renormalized parameters. For given $\mu$ and large $V_{0}$, there were no propagating states in the left half of the system and transport could only take place via a tunneling mechanism. Indeed, we could observe a residual conductance for very small systems of $N<15$ lattice sites. However, an analysis of the renormalized potential $\tilde{V}_{0}$ indicated that the potential is decreased with increasing $U$. With $U$ being chosen large enough, we could therefore obtain finite $n_{L}$ for system parameters which lead to $n_{L}=0$ for $U=0$.

The local particle densities $\left\langle n_{L, R}(j)\right\rangle$ on the left/right side of the potential step as well as the renormalized potential $\tilde{V}_{0}(j)$ exhibited strongly pronounced Friedel oscillations with frequencies determined just by the corresponding fillings $n_{L, R}$. With $n_{R}$ being independent from the interaction, only the frequency of the Friedel oscillations on the left side of the system showed a dependence on the interaction strength $U$.

The conductance was entirely suppressed in the regime of large $V_{0}$ with $n_{L}=0$, but also achieved finite values with increasing $U$. However, we were not able to enter the parameter regime in which a power law decay of the conductance could be observed. We therefore introduced an additional strong single impurity $V_{1}$ in the middle of the system (right at the potential step) in order to force the system into 
the Luttinger liquid regime. Actually, we could observe a power law decay of the conductance with increasing system size, but the exponents did not correspond to the theory for any combination of the fillings $n_{L, R}$. We obtained exponents $\beta_{L, R}$ from the local spectral functions on the left/right side of the potential step. $\beta_{R}$ fitted very well to the theoretical prediction made for a single impurity at the corresponding filling $n_{R}$, but this was not the case for $\beta_{L}$. Whereas the theory predicted an increase of $\beta_{L}$ with $V_{0}$ due to the smaller filling $n_{L}$, we found a decrease of $\beta_{L}$. Nevertheless, a linear dependence on $U$ was also observed in this case.

In the following chapter, we will shrink our system to a zero-dimensional quantum dot consisting of only $N=2$ lattice sites. The case $N=1$ is unimportant here, as $\mathrm{SOI}$ is connected to a modification of the hopping amplitudes in the system requiring at least two lattice sites to be detectable. 


\section{Chapter 6}

\section{Quantum dots of correlated electrons with SOI and magnetic field}

Quantum dots have gained much interest in the past years, in particular due to the possibility of their experimental feasibility and their possible application to nanodevices and quantum information processing [67.

A quantum dot can be realized by a single atom or molecule, but also by some semiconductor device in which a suitable electrode structure confines the electrons to a region with a diameter of $10-50 \mathrm{~nm} 68$. Due to the strong spatial confinement, the energy levels of the quantum dot are quantized and well-seperated, which allows for a treatment that only takes a few levels in the vicinity of the relevant energy scale (e.g. the chemical potential of the attached leads) into account. Ideally, the density of states of a decoupled dot is a $\delta$-function, but in reality, fluctuations in the confinement lead to a broadening.

In order to study these properties, the quantum dot is attached to higher-dimensional leads via tunneling barriers. It is experimentally achievable to design an electrode structure of metallic gates which enables the application of an additional gate voltage $V_{G}$ to control the dot's energy levels. In a simple one-particle picture, one would expect a current to flow in the zero-temperature limit, if the dot's eigenenergy matches the Fermi energy of the leads and a small source-drain voltage is impressed on the dot. This should lead to a Lorentzian peak structure in the profile of the linear conductance $G\left(V_{G}\right)$ as function of the gate voltage. The peaks are broadened due to the hybridization of the dot and the leads. However, contrary to this theory conductance measurements on quantum dots reveal conductance plateaus instead of conductance peaks in the low-temperature limit 68. This can be explained by the famous Kondo effect which dominates the physics of a quantum dot for energies below a certain energy scale, the so-called Kondo temperature $T_{K}$ (see below).

We will study the spin-dependent transport properties of a serial double quantum dot (consisting of $N=2$ lattice sites) in Sect. 6.1 and give a detailed analysis of 
the eigenvalue problem of the decoupled non-interacting dot in presence of SOI and a magnetic field. Furthermore, we will discuss the influence of SOI on the Kondo physics of the interacting dot. In Sect. 6.2 we will include the double dot in one arm of an Aharonov-Bohm interferometer and study interference effects and how the linear conductance and the spin polarization can be controlled by tuning the system parameters.

\subsection{Transport through a serial quantum dot with SOI and magnetic field}

We can directly transfer the Hamiltonian we used in the previous chapter to model the quantum wire to describe a serial quantum dot consisting of $N$ lattice sites. The lattice site index $j$ in Eqs. (3.1), (5.1) and (5.2) runs from $j=1$ to $N$ with $N=2$ for the double dot considered here. The hybridization Hamiltonian modeling the tunneling contact to the leads is given by Eq. (3.12) such that the setup is the same as in Fig. 5.1, just with a reduced number of lattice sites. The external scalar potential $V_{j, \sigma}$ in Eq. (3.3) is replaced by a common gate voltage $V_{G}$ leading to the gate Hamiltonian

$$
H_{\text {gate }}=V_{G} \sum_{j=1}^{N} \sum_{\sigma=\uparrow, \downarrow} c_{j, \sigma}^{\dagger} c_{j, \sigma} .
$$

In the following, we will tune the gate voltage $V_{G}$ and investigate the system's reaction, i.e. the modification of the linear conductance. In analogy to the linear conductance of the quantum wire, we must take the four conductance components $G_{\sigma \sigma^{\prime}}$ with $\sigma, \sigma^{\prime}=\uparrow, \downarrow$ into account. If not explicitely mentioned, the spin quantization axis is in $z$-direction. The conductance components with respect to spin quantization along the $x$ - or $y$-axis are given by Eqs. (3.28) and (3.29).

\subsubsection{Non-interacting case}

Before we start analyzing correlation effects like the Kondo effect, we focus on the situation without Coulomb interaction, i.e. $U_{1}=U_{2}=0$, and investigate the interplay of SOI and magnetic field.

Figs. 6.1 a) and b) show the total linear conductance $G_{\text {total }}$ and the conductance components $G_{\sigma \sigma^{\prime}}$ of a double $\operatorname{dot}(N=2)$ at $\mu=0$ for SOI $\alpha_{z}=0$ and $\alpha_{z}=0.5$, respectively. The dot is coupled to the two semi-infinite leads via hopping amplitudes $t_{L}=t_{R}=0.2$. Without SOI, the conductance is fully carried by the spin-conserving components $G_{\sigma \sigma}$ and shows Lorentzian peaks of width $\Gamma=t_{L}^{2}+t_{R}^{2}$ at $V_{G}= \pm t$ with conventional hopping $t=1$ according to the Breit-Wigner formula. Since no magnetic field is present, we obtain $G_{\uparrow \uparrow}=G_{\downarrow \downarrow}$. Note that the components $G_{\sigma \sigma^{\prime}}^{(x, y)}$ are also degenerate with respect to the spin quantization axis in this case. For finite SOI $\left(\alpha_{y}=0, \alpha_{z}=0.5\right)$, the peaks are shifted to $V_{G}= \pm \sqrt{t^{2}+\alpha_{z}^{2}}$, but the peak width remains unchanged. A certain fraction of the total conductance is carried 

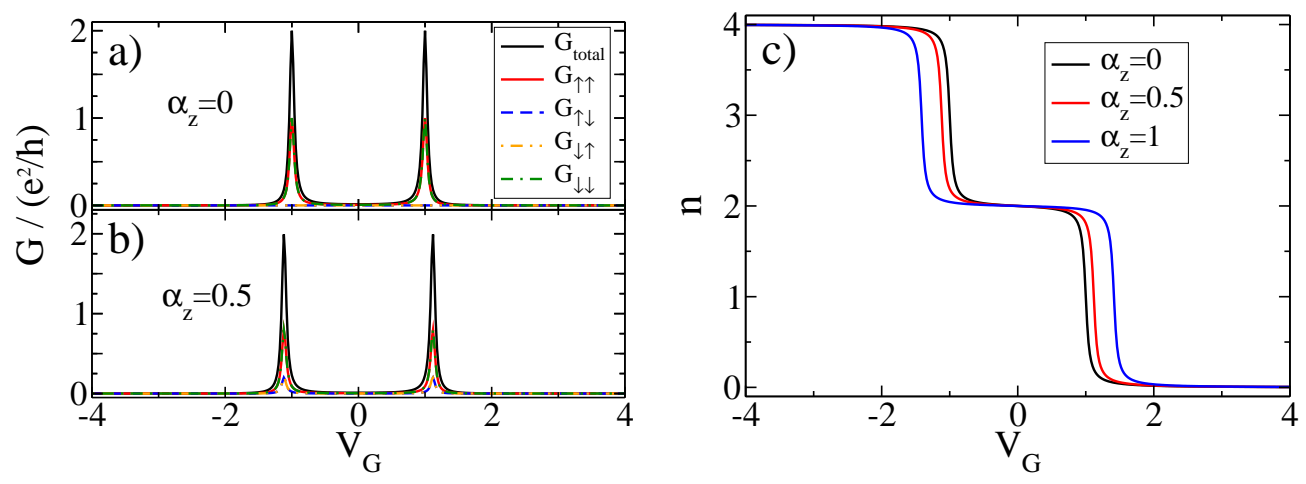

Figure 6.1: Linear conductance and particle number of a double quantum dot $(N=2)$ coupled to semi-infinite leads via $t_{L}=t_{R}=0.2$ as a function of gate voltage $V_{G}$.

a) Without SOI, the conductance is fully carried by the spin-conserving components $G_{\sigma \sigma}$ and shows Lorentzian peaks of width $\Gamma=t_{L}^{2}+t_{R}^{2}$ at $V_{G}= \pm t$ with hopping amplitude $t=1$.

b) For $\alpha_{z}=0.5\left(\alpha_{y}=0\right)$, the peaks are shifted to $V_{G}= \pm \sqrt{t^{2}+\alpha_{z}^{2}}$, but their width remains unchanged. A small fraction of the total conductance is carried by the spin-flip components $G_{\sigma-\sigma}$.

c) The particle number $n$ of the double dot shows a steplike behavior with the position of odd particle numbers matching the corresponding conductance peak positions.

by the spin-flip components $G_{\sigma-\sigma}$. A thorough analysis shows that this fraction strongly depends on the direction with respect to which the spins are 'measured'. For arbitrary hopping amplitudes $\alpha_{y}, \alpha_{z}$ and $t$, we obtain

$$
\begin{aligned}
& G_{\uparrow \downarrow}^{(x)}\left(V_{G}\right)=G_{\downarrow \uparrow}^{(x)}\left(V_{G}\right)=\frac{\alpha^{2}}{2 t_{\text {eff }}^{2}} G_{\text {total }}\left(V_{G}\right) \\
& G_{\uparrow \downarrow}^{(y)}\left(V_{G}\right)=G_{\downarrow \uparrow}^{(y)}\left(V_{G}\right)=\frac{\alpha_{z}^{2}}{2 t_{\text {eff }}^{2}} G_{\text {total }}\left(V_{G}\right) \\
& G_{\uparrow \downarrow}^{(z)}\left(V_{G}\right)=G_{\downarrow \uparrow}^{(z)}\left(V_{G}\right)=\frac{\alpha_{y}^{2}}{2 t_{\text {eff }}^{2}} G_{\text {total }}\left(V_{G}\right)
\end{aligned}
$$

with $\alpha=\sqrt{\alpha_{y}^{2}+\alpha_{z}^{2}}$ and $t_{\text {eff }}=\sqrt{t^{2}+\alpha_{y}^{2}+\alpha_{z}^{2}}$ being already defined in Sect. 3.3, This is in correspondence with the intuitive picture already discussed in the continuum case that the SOI parameters $\alpha_{y}\left(\alpha_{z}\right)$ rotate the spin into the $z$ - $(y$ - $)$ direction to a certain amount which depends on the relative fraction of their absolute values. We emphasize this fact, since it might be crucial for the spatial measurement geometry, e.g. Stern-Gerlach apparatus, in order to detect spin polarization in experiments.

In contrast to the previous chapter in which $n$ denoted the normalized particle 

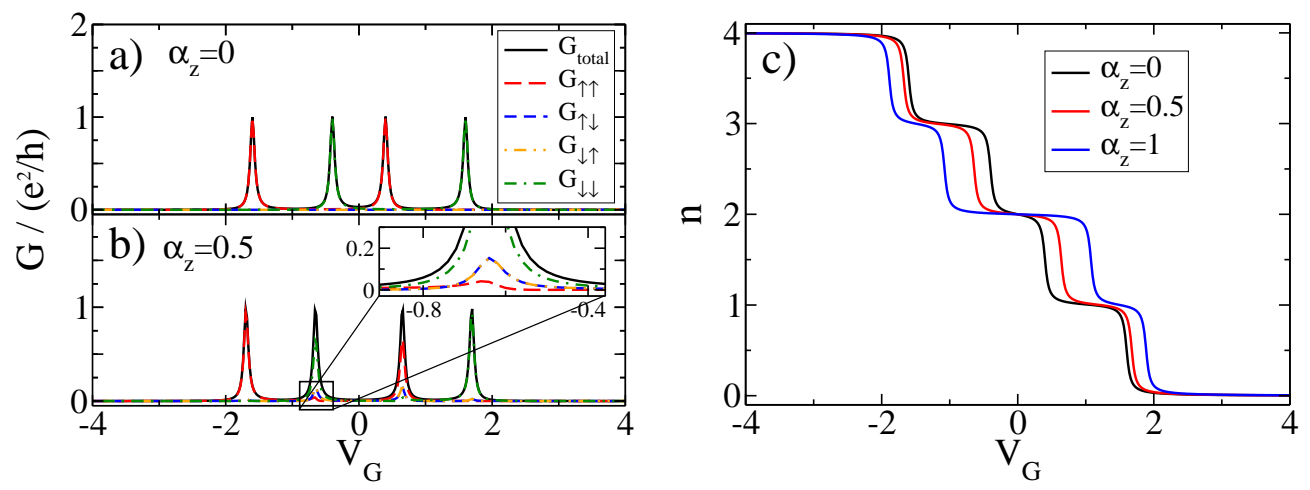

Figure 6.2: Linear conductance and particle number of a double quantum dot with the same parameters as in Fig. 6.1] but finite magnetic field $\gamma B_{z}=0.6$.

a) Without SOI, the spin degeneracy is lifted which leads to a splitting of the conductance peaks to $V_{G}= \pm\left(t \pm \gamma B_{z}\right)$. The peak width is not altered by the magnetic field.

b) For $\alpha_{z}=0.5\left(\alpha_{y}=0\right)$, the peaks are positioned at $V_{G}= \pm \sqrt{\left(t \pm \gamma B_{z}\right)^{2}+\alpha_{z}^{2}}$. The spin-flip components $G_{\sigma-\sigma}$ are still degenerate, but have less impact on the outer peaks than on the inner peaks.

c) The steplike shape of the particle number $n$ is subdivided by the magnetic field and a change of $n$ by one electron corresponds to a conductance peak.

number, i.e. the filling of the quantum wire, in this chapter it is defined via

$$
n=\sum_{j=1}^{N} \sum_{\sigma=\uparrow, \downarrow}\left\langle n_{j, \sigma}\right\rangle
$$

and therefore counts the total number of electrons on the dot. Due to the Pauli principle, $n \in[0,2 N]$ holds for a dot of $N$ lattice sites.

The particle number $n$ as a function of $V_{G}$ is shown in Fig. 6.1] c) for $\alpha_{z}=0,0.5,1$. $n$ shows characteristic plateaus leading to a steplike shape. A steep gradient of $n$ is observed at $V_{G}= \pm t_{\text {eff. }}$ It follows from Fig. 6.1 a) and b) that we obtain a conductance peak, whenever the dot's occupation number $n$ changes. Without an external magnetic field, i.e. in the spin-degenerate case, $n$ always changes by 2 . Therefore, the plateaus only occur for even particle numbers. As mentioned in the introduction to this chapter, a change in the dot's occupancy can be expected, whenever the gate voltage passes over an eigenenergy of the dot. Before we analyze this in detail, we will shortly investigate the behavior of an external magnetic field and address the eigenvalue problem afterwards in general.

We account for an external magnetic field $\mathbf{B}=\left(B_{x}, B_{y}, B_{z}\right)$ applied to the dot by a Zeeman Hamiltonian as given in Eq. (3.6). Fig. 6.2 a) shows the influence of the magnetic field $\gamma B_{z}=0.6$ on the conductance components for the case without SOI. The spin degeneracy is lifted, but we obtain the symmetry $G_{\uparrow \uparrow}\left(V_{G}\right)=G_{\downarrow \downarrow}\left(-V_{G}\right)$. As 
expected, there are four conductance peaks at $V_{G}= \pm\left(t \pm \gamma B_{z}\right)$, two are carried by $G_{\uparrow \uparrow}$ and the other two by $G_{\downarrow \downarrow}$. The spin-flip components come into play for finite SOI as shown in Fig. 6.2 b) for $\alpha_{z}=0.5\left(\alpha_{y}=0\right)$ and are still degenerate, $G_{\uparrow \downarrow}\left(V_{G}\right)=$ $G_{\downarrow \uparrow}\left(V_{G}\right)$. The peaks are positioned at $V_{G}= \pm \sqrt{\left(t \pm \gamma B_{z}\right)^{2}+\alpha_{z}^{2}}$, i.e. $B_{z}$ is only coupled to $t$ and not to $\alpha_{z}$. The inset shows that, although the total conductance is mainly carried by $G_{\downarrow \downarrow}, G_{\uparrow \uparrow}$ also shows a maximum which is not present in the case without SOI. The spin-flip components are much more pronounced in the two inner peaks, which will be explained below.

The particle number $n$ for magnetic field $\gamma B_{z}=0.6$ and SOI parameters $\alpha_{z}=$ $0,0.5,1\left(\alpha_{y}=0\right)$ are shown in Fig. 6.2 $\left.\mathrm{c}\right)$. In contrast to the case without magnetic field, we find a subdivision of the steps such that plateaus also occur for odd occupancy numbers. Again, a change in $n$ is linked to a conductance peak in Fig. 6.2 a) and b) and the plateaus correspond to the almost vanishing conductance in between. In order to analyze all these observations in more detail, we now focus on the eigenvalue problem of the double dot.

\section{The double dot's eigenvalue problem}

Since we omit the Coulomb interaction in the present setup, the one-particle Hamiltonian of the isolated double dot (without the leads) can be written as a complex $4 \times 4$-matrix $\bar{H}$ in the lattice-spin representation of Wannier states $|j, \sigma\rangle$ [see Sect. 3.1. In its most general form with chemical potential $\mu$, arbitrary magnetic field $\mathbf{B}=\left(B_{x}, B_{y}, B_{z}\right)$, conventional hopping amplitude $t$ and SOI parameters $\alpha_{y}$ and $\alpha_{z}$ this matrix is given by

$$
\bar{H}=\left(\begin{array}{cccc}
\mu+\gamma B_{z} & \gamma\left(B_{x}-i B_{y}\right) & -t+i \alpha_{y} & \alpha_{z} \\
\gamma\left(B_{x}+i B_{y}\right) & \mu-\gamma B_{z} & -\alpha_{z} & -t-i \alpha_{y} \\
-t-i \alpha_{y} & -\alpha_{z} & \mu+\gamma B_{z} & \gamma\left(B_{x}-i B_{y}\right) \\
\alpha_{z} & -t+i \alpha_{y} & \gamma\left(B_{x}+i B_{y}\right) & \mu-\gamma B_{z}
\end{array}\right)
$$

The Hermitian structure of $\bar{H}$ guarantees that the four eigenvalues

$$
\begin{aligned}
& \lambda_{1}=\mu+\sqrt{\alpha^{2}+t^{2}+\gamma^{2} B^{2}+2 \gamma \sqrt{t^{2} B^{2}+\left(\alpha_{y} B_{z}+\alpha_{z} B_{y}\right)^{2}}} \\
& \lambda_{2}=\mu+\sqrt{\alpha^{2}+t^{2}+\gamma^{2} B^{2}-2 \gamma \sqrt{t^{2} B^{2}+\left(\alpha_{y} B_{z}+\alpha_{z} B_{y}\right)^{2}}} \\
& \lambda_{3}=\mu-\sqrt{\alpha^{2}+t^{2}+\gamma^{2} B^{2}-2 \gamma \sqrt{t^{2} B^{2}+\left(\alpha_{y} B_{z}+\alpha_{z} B_{y}\right)^{2}}} \\
& \lambda_{4}=\mu-\sqrt{\alpha^{2}+t^{2}+\gamma^{2} B^{2}+2 \gamma \sqrt{t^{2} B^{2}+\left(\alpha_{y} B_{z}+\alpha_{z} B_{y}\right)^{2}}}
\end{aligned}
$$

with $\alpha=\sqrt{\alpha_{y}^{2}+\alpha_{z}^{2}}$ and $B=\sqrt{B_{x}^{2}+B_{y}^{2}+B_{z}^{2}}$ are real. The most interesting feature of Eq. (6.5) is that $\alpha_{y}$ couples directly to $B_{z}$ and $\alpha_{z}$ to $B_{y}$ in the last term. However, this interplay could have been guessed from the fact that $\alpha_{y}\left(\alpha_{z}\right)$ leads to a spinrotation into the $z$ - $(y$ - $)$ direction, i.e. the direction in which $B_{z}\left(B_{y}\right)$ induces a spin 


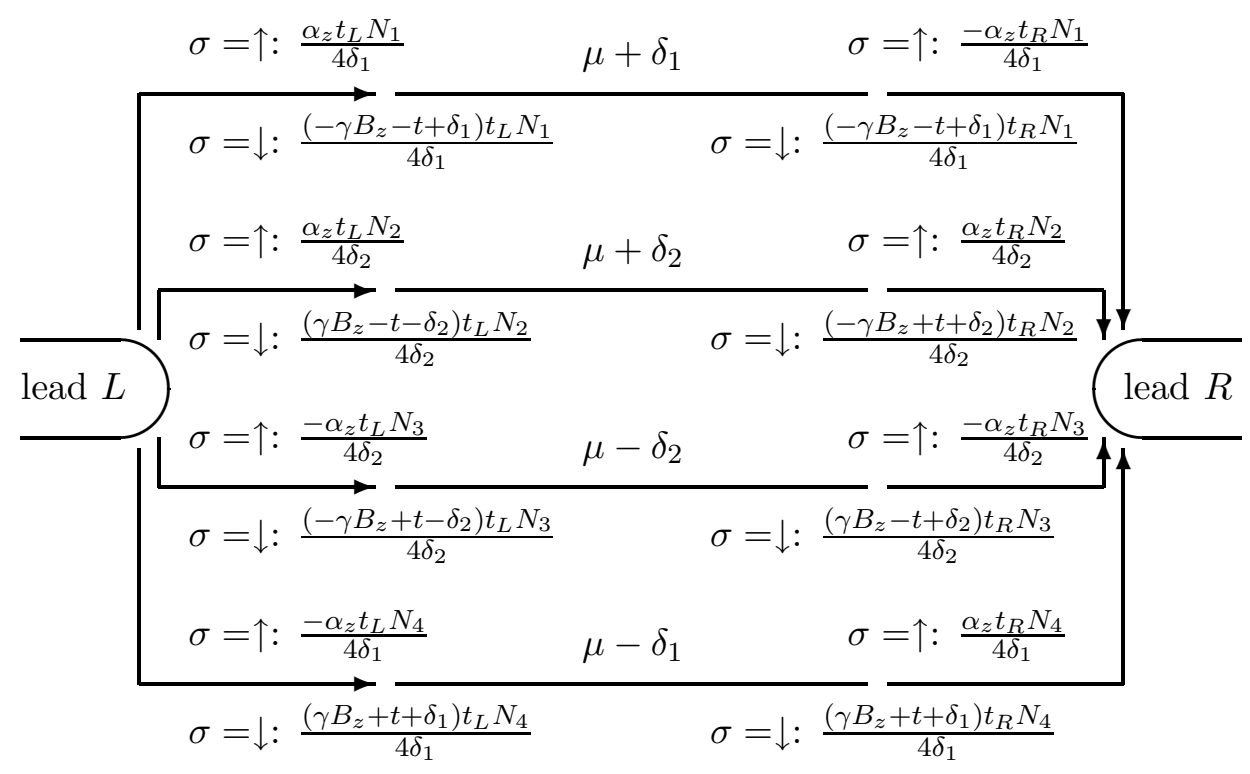

Figure 6.3: Coupling between the leads and the four energy levels $\mu \pm \delta_{1,2}$ of the double dot in energy representation. The effective hopping amplitudes $t_{i}^{L, R}(\sigma)$ depend on the energy level and the spin direction.

splitting effect. The eigenvectors of $\bar{H}$ can be obtained easily by a computer algebra program, but are too cumbersome to write down here.

We therefore concentrate on the special situation shown in Fig. 6.2 with $\alpha_{y}=0$ and $B_{x}=B_{y}=0$. Defining

$$
\begin{aligned}
& \delta_{1}=\sqrt{\alpha_{z}^{2}+\left(t+\gamma B_{z}\right)^{2}} \\
& \delta_{2}=\sqrt{\alpha_{z}^{2}+\left(t-\gamma B_{z}\right)^{2}},
\end{aligned}
$$

the eigenvalues simplify to

$$
\begin{aligned}
& \lambda_{1}=\mu+\delta_{1} \\
& \lambda_{2}=\mu+\delta_{2} \\
& \lambda_{3}=\mu-\delta_{2} \\
& \lambda_{4}=\mu-\delta_{1} .
\end{aligned}
$$

The eigenvectors and further explanations are given in App. [C] Fig. 6.3 shows the coupling of the leads to the four energy levels $\mu \pm \delta_{1,2}$ of the double dot in the energy representation [for a definition of $N_{i}$ see App. C]. The amplitude of the effective hybridization depends on the corresponding energy level $\lambda_{i}$ and on the spin direction $\sigma=\uparrow, \downarrow$. Note, however, that no spin can be assigned to electrons on a certain energy level due to the base transformation, but the spin quantum number 
$\sigma$ only refers to electrons in the leads. It is shown in App. [C] that the two outer levels $\lambda_{1}=\mu+\delta_{1}$ and $\lambda_{4}=\mu-\delta_{1}$ are coupled to each other via hopping processes induced by $t$ and $\alpha_{z}$. The same holds for the inner levels $\lambda_{2}=\mu+\delta_{2}$ and $\lambda_{3}=\mu-\delta_{2}$. Interestingly, there is no coupling between the inner and the outer levels.

For a single dot without SOI, it has been shown by Meir and Wingreen in Ref. 69 that the conductance can also be derived directly from the spectral weight of the dot at the chemical potential. This approach also applies for the case with Coulomb interaction and has been discussed in Ref. 20 within the fRG framework. However, as it is not clear, how this could be transferred quantitatively to our much more complicated situation (even without Coulomb interaction), we just take some qualitative considerations into account to explain the behavior of the conductance components in Fig. 6.2. We define the hybridizations

$$
\Gamma_{i}^{L, R}(\sigma)=\left|t_{i}^{L, R}(\sigma)\right|^{2}
$$

between the left/right lead and the $i$-th energy level $\lambda_{i}$. Since we assumed symmetric coupling to the leads in the lattice-spin base, i.e. $t_{L}=t_{R}$, we obtain $\Gamma_{i}^{L}(\sigma)=\Gamma_{i}^{R}(\sigma)$ from Fig. 6.3. Using the same values as in Fig. 6.2. i.e. $\mu=0, t=1, \alpha_{z}=0.5$ and $\gamma B_{z}=0.6$, we get

$$
\begin{array}{llrl}
\Gamma_{1}^{L}(\uparrow) & =\Gamma_{1}^{R}(\uparrow)=0.49, & & \Gamma_{1}^{L}(\downarrow)=\Gamma_{1}^{R}(\downarrow)=0.01 \\
\Gamma_{2}^{L}(\uparrow)=\Gamma_{2}^{R}(\uparrow)=0.09, & & \Gamma_{2}^{L}(\downarrow)=\Gamma_{2}^{R}(\downarrow)=0.41 \\
\Gamma_{3}^{L}(\uparrow)=\Gamma_{3}^{R}(\uparrow)=0.41, & & \Gamma_{3}^{L}(\downarrow)=\Gamma_{3}^{R}(\downarrow)=0.09 \\
\Gamma_{4}^{L}(\uparrow)=\Gamma_{4}^{R}(\uparrow)=0.01, & & \Gamma_{4}^{L}(\downarrow)=\Gamma_{4}^{R}(\downarrow)=0.49 .
\end{array}
$$

Obviously, the symmetry $\lambda_{1}=-\lambda_{4}$ and $\lambda_{2}=-\lambda_{3}$ leads to a symmetry $\Gamma_{1}^{L, R}(\sigma)=$ $\Gamma_{4}^{L, R}(-\sigma)$ and $\Gamma_{2}^{L, R}(\sigma)=\Gamma_{3}^{L, R}(-\sigma)$. Furthermore, all energy levels are equally coupled to the leads, if we take into account the sum of the respective $(\sigma=\uparrow)$ - and $(\sigma=\downarrow)$-hybridizations. Let us assume in a naive picture that the linear conductance $G_{\sigma \sigma^{\prime}}$ via the energy level $\lambda_{i}$ is proportional to the product $\Gamma_{i}^{L}(\sigma) \Gamma_{i}^{R}\left(\sigma^{\prime}\right)$, which can actually be taken as a modified statement from Ref. 69. We obtain for the two inner levels $\left(\lambda_{2}\right.$ and $\left.\lambda_{3}\right) \Gamma_{2,3}^{L}(\sigma) \Gamma_{2,3}^{R}(-\sigma)=0.037$ in comparison to $\Gamma_{1,4}^{L}(\sigma) \Gamma_{1,4}^{R}(-\sigma)=0.005$ for the outer levels $\left(\lambda_{1}\right.$ and $\left.\lambda_{4}\right)$. Thus, it is quite reasonable to expect the spin-flip components $G_{\sigma-\sigma}$ to be much more pronounced at $V_{G}=\lambda_{2,3}$ than at $V_{G}=\lambda_{1,4}$. The same argument explains the alternation of $G_{\uparrow \uparrow}$ and $G_{\downarrow \downarrow}$ in contributing to the four peaks at $V_{G}=\lambda_{i}$.

Naturally, this view lacks from disregarding any coupling between the different energy levels. However, it is shown in App. C that the inner levels are decoupled from the outer levels. The coupling between $\lambda_{1}$ and $\lambda_{4}$ as well as between $\lambda_{2}$ and $\lambda_{3}$ might achieve reasonable values, but transport via different energy levels at a fixed gate voltage can be omitted, as the level spacing is too large to allow for two energy levels being close to the Fermi energy of the leads simultaneously. 


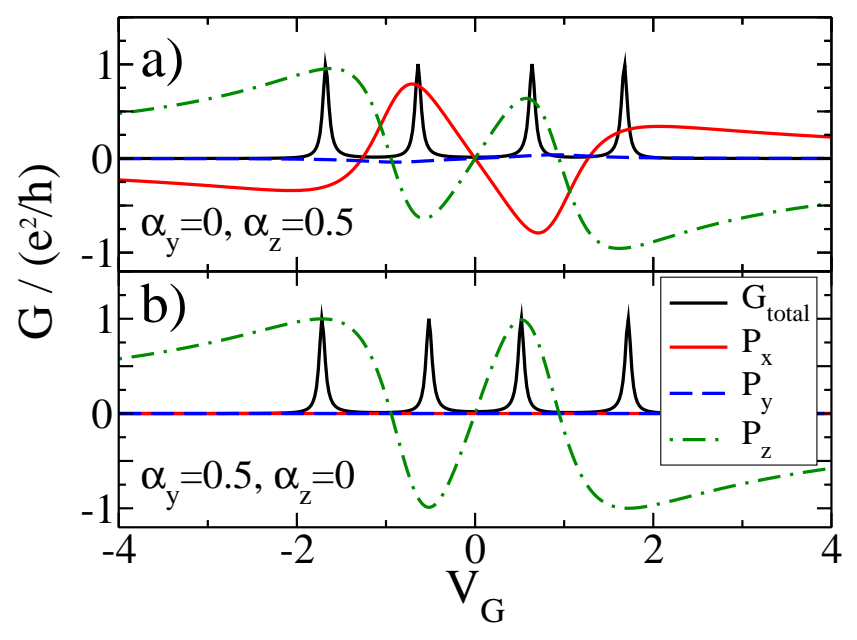

Figure 6.4: Total conductance and spin polarization of a double dot as a function of the gate voltage $V_{G}$ for $\gamma B_{z}=0.6$.

a) For $\alpha_{y}=0$ and $\alpha_{z}=0.5$ the spins are partially rotated from the $z$-direction into the $x$-direction. We observe oscillations and a point symmetry with respect to $\left(V_{G}, P_{i}\right)=(0,0)$ of all polarization components, but the magnitude of $P_{y}$ can be disregarded. $P_{x}$ and $P_{z}$ reveal sizeable values, especially close to the conductance peaks.

b) For $\alpha_{y}=0.5$ and $\alpha_{z}=0, P_{x}=P_{y}=0$ vanish for all $V_{G}$ and we obtain perfect spin polarization $P_{z}= \pm 1$ at the conductance peaks.

\section{Spin polarization}

We now analyze the spin-polarization of the linear current through the double dot. The polarization vector $\mathbf{P}=\left(P_{x}, P_{y}, P_{z}\right)$ for the double dot is defined in the same way as for the quantum wire, i.e. via the conductance components $G_{\sigma \sigma^{\prime}}^{(x, y, z)}$ [see Eqs. (3.27), (3.28) and (3.29)]. We have observed the degeneracies $G_{\uparrow \uparrow}\left(V_{G}\right)=G_{\downarrow \downarrow}\left(V_{G}\right)$ and $G_{\uparrow \downarrow}\left(V_{G}\right)=G_{\downarrow \uparrow}\left(V_{G}\right)$ in Fig. 6.1 for the case without a magnetic field and just SOI being present. Thus, one cannot expect a spin-polarized current.

Fig. 6.4 a) shows the total conductance and the spin-polarization for the same parameters as in Fig. 6.2 b), i.e. with a finite magnetic field $\gamma B_{z}=0.6$. Due to the finite SOI $\alpha_{z}=0.5$, the spins are rotated partially out of the $z$-direction, which is preferred due to the magnetic field $B_{z}$. Interestingly and in contrast to the situations studied in Sect. 3.4. the finite $\alpha_{z}$ does not rotate the spin into the $y$-direction but into the $x$-direction. The interplay of $\alpha_{z}$ and $B_{z}$ leads to an almost perfect degeneracy $G_{\uparrow \uparrow}^{(y)}=G_{\downarrow \downarrow}^{(y)}$ and $G_{\uparrow \downarrow}^{(y)}=G_{\downarrow \uparrow}^{(y)}$ suppressing any sizeable spin polarization $P_{y}$. Although $G_{\uparrow \uparrow}^{(x)}=G_{\downarrow \downarrow}^{(x)}$ holds for the same parameters, we observe a large difference between $G_{\uparrow \downarrow}^{(x)}$ and $G_{\downarrow \uparrow}^{(x)}$ close to the conductance peaks which leads to the pronounced 
polarization $P_{x}$. We find for the total polarization $P=\sqrt{P_{x}^{2}+P_{y}^{2}+P_{z}^{2}}=1$ at the conductance peaks, i.e. a direction exists in which perfect spin polarization can be obtained.

In Fig. 6.4 b), we rotate the Rashba field into the $y$-direction, i.e. $\alpha_{y}=0.5$ and $\alpha_{z}=0$, and obtain perfect spin polarization $P_{z}= \pm 1$ at the conductance peaks, whereas $P_{x}=P_{y}=0$ for all $V_{G}$.

One should keep in mind that a sizeable spin polarization does not automatically lead to a sizeable spin polarized current, especially in situations in which the total conductance shows a pronounced peak structure. Thinking of spin transistors, the product of spin polarization and total conductance is a more meaningful quantity for applications. Although the spin polarization in Fig. 6.4 is sizeable in quite a large neighborhood of the peaks, a gate voltage in just a very small interval around the conductance peaks would lead to useful spin currents.

\subsubsection{Coulomb interaction}

We now include the Coulomb interaction in our model via

$$
H_{1}=U_{1} \sum_{j=1}^{N} \sum_{\sigma, \sigma^{\prime}} c_{j, \sigma}^{\dagger} c_{j, \sigma} c_{j, \sigma^{\prime}}^{\dagger} c_{j, \sigma^{\prime}}\left(1-\delta_{\sigma, \sigma^{\prime}}\right)
$$

for the local part $U_{1}$ and

$$
H_{2}=U_{2} \sum_{j=1}^{N} \sum_{\sigma, \sigma^{\prime}} c_{j+1, \sigma}^{\dagger} c_{j+1, \sigma} c_{j, \sigma^{\prime}}^{\dagger} c_{j, \sigma^{\prime}}
$$

for the nearest-neighbor part $U_{2}$.

As shown for quantum dots without SOI in Ref. 20 by comparison to results obtained by NRG, the fRG method provides very good results, if one takes into account the flow of the two-particle vertex. For quantum dots of up to $N=3$ lattice sites, it is numerically feasible to account for the fRG flow of the two-particle vertex $\gamma_{2}^{\Lambda}$ according to Eq. (4.23) without any further parametrization, even for finite SOI and magnetic field. Therefore, we will use this scheme in the following. Fig. 6.5 shows the conductance components $G_{\sigma \sigma^{\prime}}$ and occupation number $n$ for the same parameters as in Fig. 6.1, but finite Coulomb interaction $U_{1}=1$ and $U_{2}=0.5$. In Fig. 6.5 a), one observes a characteristic broadening of the Lorentzian peaks which have been observed in the non-interacting case [see Fig. 6.1]. This broadening is due to the Kondo effect, which occurs for an odd number of electrons on the dot. The magnetic moments (spins) of the lead electrons interact collectively with the localized ("pinned") spin on the dot forming an effective non-magnetic ground state, which leads to an enhanced conductance [70. Furthermore, the Kondo plateaus are shifted to larger $\left|V_{G}\right|$ in comparison to the Lorentzian peaks in the non-interacting case, which can be explained by the renormalization of the effective hopping. As can be seen from Fig. 6.5 b), finite SOI does not destroy the Kondo resonances. This has 

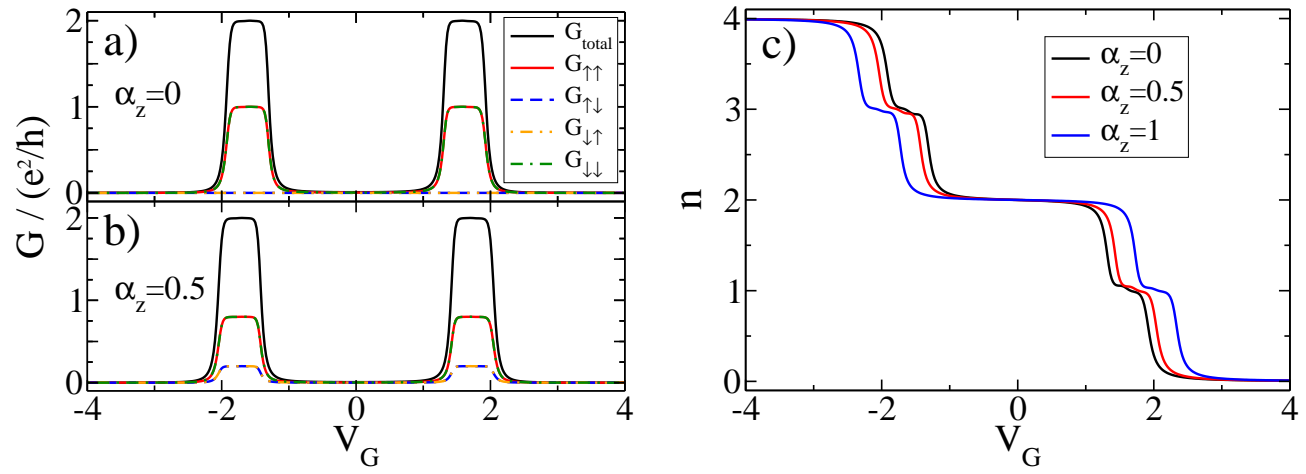

Figure 6.5: Linear conductance and particle number of a double quantum dot with the same parameters as in Fig. 6.1] but finite Coulomb interaction $U_{1}=1$ and $U_{2}=0.5$.

a) The conductance peaks observed in the non-interacting case are broadened to characteristic plateaus.

b) The plateaus are shifted towards larger $\left|V_{G}\right|$ due to SOI. The spin-flip components $G_{\sigma-\sigma}$ give a finite contribution to the total conductance.

c) The finite Coulomb interaction leads to the formation of plateaus for odd occupation numbers $n$. These plateaus are driven to larger $\left|V_{G}\right|$, if the effective hopping via SOI parameters $\alpha_{z}=0,0.5,1$ is increased.

already been pointed out in Refs. [38, 71] and has also been observed in experiments [72. Nevertheless, this is a quite astonishing result, as the spin is not a conserved physical quantity on the dot in presence of SOI and, therefore, it is not clear how spin-scattering as a collective many-body effect can take place on the dot in order to reveal a Kondo resonance. However, as shown in App. A the SOI does not lead to finite spin-flip components of the local Green's function which might explain the "survival" of the Kondo effect in presence of SOI.

The ratio of spin-conserving and spin-flipping components is not altered due to Coulomb interaction, but Eq. (6.2) still holds. This means that the conventional and the SOI hopping are renormalized by the same factor.

Fig. 6.5 c) shows the effect of the SOI $\left(\alpha_{z}=0,0.5,1\right)$ on the particle number for $U_{1}=1, U_{2}=0.5$. The formation of plateaus at odd occupation numbers is not hampered by the SOI. The plateaus are just driven to larger $\left|V_{G}\right|$ due to the increased effective hopping.

\section{Kondo temperature}

We now account for a finite magnetic field $\mathbf{B}=\left(0,0, B_{z}\right)$. Fig. 6.6 a) shows the conductance components of a system with the same parameters as in Fig. 6.5 b), but $\gamma B_{z}=0.6$. The Kondo effect is destroyed and each plateau has split up into two well-separated conductance peaks. In comparison to Fig. 6.2 b) in which the same 

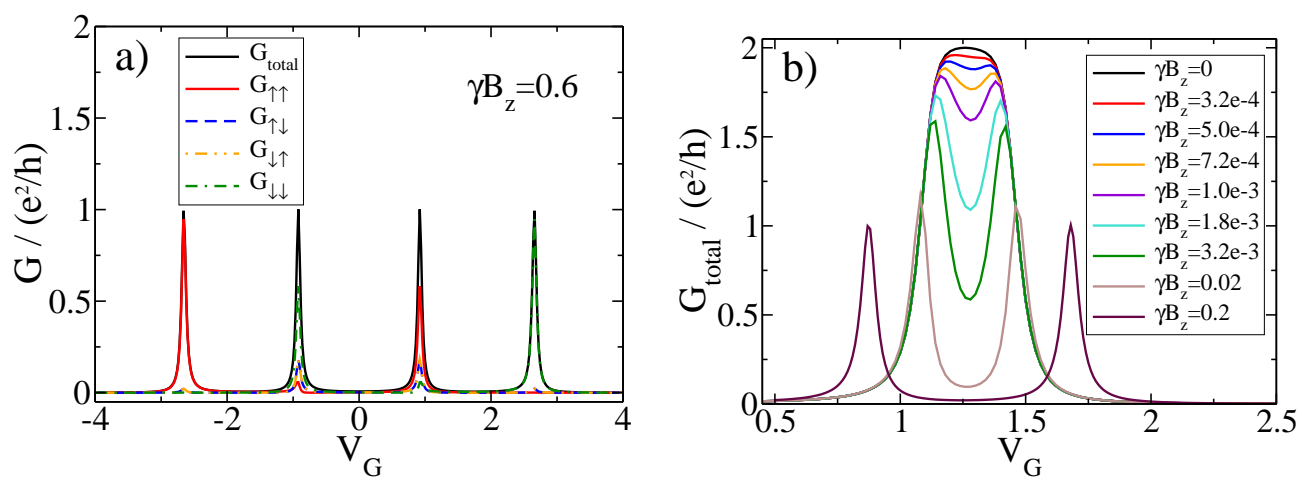

Figure 6.6: Influence of a magnetic field $\gamma B_{z}$ on the conductance of a double dot. a) Conductance components for parameters $\alpha_{z}=0.5, \gamma B_{z}=0.6, U_{1}=1$ and $U_{2}=0.5$. The Kondo plateaus split up into two separated conductance peaks.

b) For small magnetic fields, one observes a continuously increasing dip in the plateau region (here for $U_{1}=1, U_{2}=0$ and no $\mathrm{SOI}$ ).

parameters were chosen except for the finite Coulomb interaction, one observes that all peaks are shifted to larger $\left|V_{G}\right|$ due to the increased renormalized hopping. The separation of the peaks at positive/negative $V_{G}$ also has increased by a factor of 1.7 indicating that the magnetic field also has been renormalized significantly due to the Coulomb interaction. The peak position and the particular contribution of the components to the total conductance can be understood in the same way as in the non-interacting case [see Sect. 6.1.1], but with renormalized system parameters.

Fig. 6.6 b) shows that the Kondo effect is destroyed continuously with increasing magnetic field. It seems as if $\gamma B_{z}$ has to exceed a certain critical value in order to drive the conductance peaks out of the plateau region.

The magnetic field $\gamma B_{z}$, which causes a dip to $G_{\text {total }}=1 e^{2} / h$, defines the characteristic energy scale of our system and corresponds to the Kondo temperature $T_{K}$. It has been shown in Ref. [20] for a single quantum dot without SOI that this is a suitable approach and yields the same behavior of $T_{K}$ as obtained by NRG (in the NRG approach, $T_{K}$ is obtained from the width of the spectral funcion).

In the following, we determine $T_{K}$ as a function of $U_{1}, U_{2}$ and $\alpha$ (since we just consider the total conductance, the ratio $\alpha_{y} / \alpha_{z}$ has no influence). Fig. 6.7 shows the Kondo temperature as a function of $U_{1}$ for parameters $U_{2}$ and $\alpha$. The logarithmic plot reveals an exponential behavior

$$
T_{K}=A \exp \left(-C U_{1} / \Gamma\right)
$$

with $\Gamma=t_{L}^{2}+t_{R}^{2}$. $A$ and $C$ are parameters which are independent of $U_{1}$. The analogy of the original Kondo model to the single impurity Anderson model (corresponding to a single quantum dot without SOI in our setup) has been shown by Schrieffer and Wolff (so-called Schrieffer-Wolff transformation) [73. The Kondo problem has been 


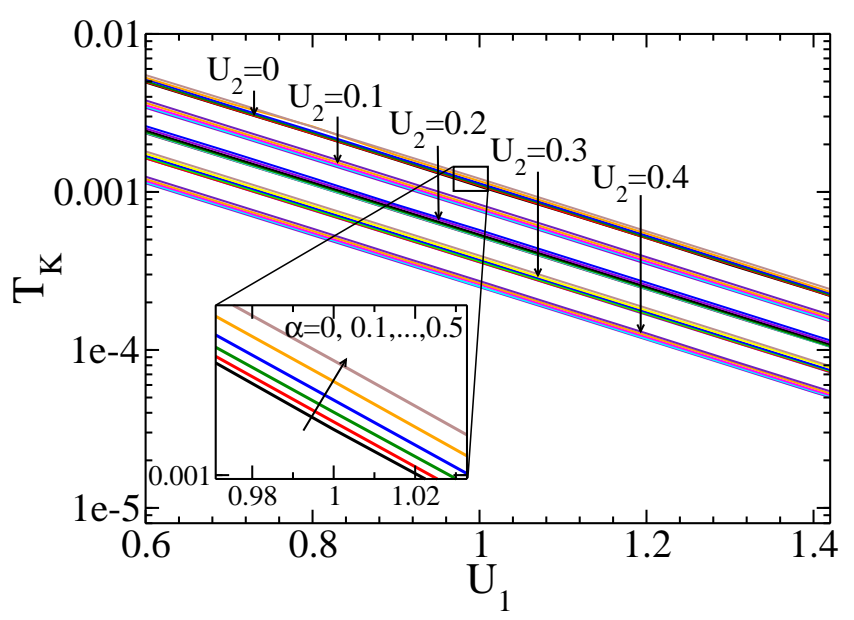

Figure 6.7: Kondo temperature $T_{K}$ of a double dot as a function of $U_{1}$ for different $U_{2}$ and $\alpha$ and $t=1, t_{L}=t_{R}=0.2$. One observes an exponential behavior $T_{K} \sim$ $\exp \left(-C U_{1} / \Gamma\right), C=$ const.

solved exactly by Bethe ansatz for the single impurity Anderson model yielding a prefactor $C=\pi / 8$ in first order $\mathcal{O}\left(U_{1}\right)$ [70. However, this result has to be modified to $C=1 / \pi$ within our fRG approximation. Thus, we obtain a theoretical value of $C_{\text {th }}=0.32$. Fitting the results in Fig. 6.7 yields $C=0.30$ and thus a very good correspondence to the theory.

The prefactor $A$ in Eq. (6.12) and its dependence on the nearest-neighbor interaction $U_{2}$ is determined in Fig. 6.8 The data can be fitted by an exponential law

$$
A=A_{0} \exp \left(-D U_{2} / \Gamma\right)
$$

with $A_{0}$ and $D$ being independent of $U_{2}$. We obtain $D=0.29$ being only slightly smaller than the constant $C$. The prefactor $A_{0}$ in Eq. (6.1.2) reveals a quadratic dependence on the SOI coupling at least for small $\alpha$. With $p\left(\alpha^{2}\right)$ being the polynomial of second order fitting the prefactor $A_{0}$ we can write the Kondo temperature as

$$
T_{K}=p\left(\alpha^{2}\right) \exp \left[-\left(C U_{1}+D U_{2}\right) / \Gamma\right] .
$$

It follows that a larger (renormalized) magnetic field is neccessary to cause a conductance dip to $G_{\text {total }}=1 e^{2} / h$ within the Kondo plateau, if $\alpha$ and, consequently, the effective hopping $t_{\text {eff }}=\sqrt{t^{2}+\alpha^{2}}$ is increased. Considering the non-interacting case, the eigenvalues $\mu \pm \delta_{1,2}$ [see Eq. [6.7]] define the effective energy scale of the system. As can be seen from Fig. 6.7, $T_{K}$ and, therefore, the (unrenormalized) magnetic field is very small in the Kondo regime, i.e. $\gamma B_{z}<0.01$. Using $\mu=0$ in our case, the eigenvalues and, consequently, the energy scale can be approximated by $\epsilon_{s}=\sqrt{t^{2}+\alpha^{2}}$. Assuming that the effective magnetic field is given by the ratio 


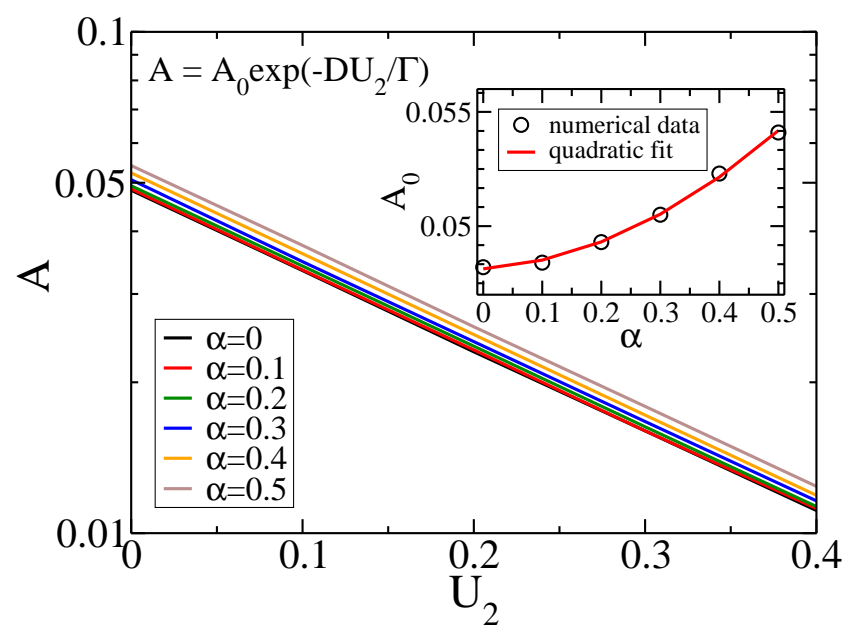

Figure 6.8: Prefactor $A$ in $T_{K}=A \exp \left(-C U_{1} \Gamma\right)$ as a function of $U_{2}$ for different $\alpha$. The data reveal an exponential dependence on $U_{2}$ and a quadratic dependence on $\alpha$.

$\gamma B_{z} / \epsilon_{s}$, this can be expanded to

$$
\frac{\gamma B_{z}}{\epsilon_{s}}=\frac{\gamma B_{z}}{\sqrt{t^{2}+\alpha^{2}}}=\frac{\gamma B_{z}}{t}\left[1-\frac{\alpha^{2}}{t^{2}}+\mathcal{O}\left(\frac{\alpha^{4}}{t^{4}}\right)\right] .
$$

For the parameters $t$ and $\alpha$ chosen here, we can truncate this series after the quadratic term, which confirms our results obtained in Fig. 6.8. Going back to the case with Coulomb interaction, one could simply try to use this explanation with the Coulomb interaction being accounted for by taking the renormalized system parameters at the end of the fRG flow. However, the Kondo effect cannot be explained in such a one-particle picture. Therefore, the explanation above should not be taken too literally, but more as an rough estimation.

As far as we know, there are neither analytical nor numerical solutions of the Kondo problem of a double dot problem with finite $U_{1}, U_{2}$ and SOI. Nevertheless, the exponential dependence of the Kondo temperature on $U_{2}$ and the quadratic dependence of the prefactor $D$ on the SOI coupling $\alpha$ obtained in our approach both seem reasonable.

\section{Spin polarization}

Investigating the influence of the Coulomb interaction on the spin polarization, we concentrate on two different situations. Fig. 6.9 a) shows the total conductance $G_{\text {total }}$ and spin polarization $P_{i}$ as a function of the gate voltage $V_{G}$ for a double dot with Coulomb interaction $U_{1}=1$ and $U_{2}=0.5$ and SOI $\alpha_{y}=0$ and $\alpha_{z}=0.5$. The magnetic field $\gamma B_{z}=0.004$ is chosen such that the system is just driven out of the Kondo regime. Both Kondo plateaus have been transformed into two separate 


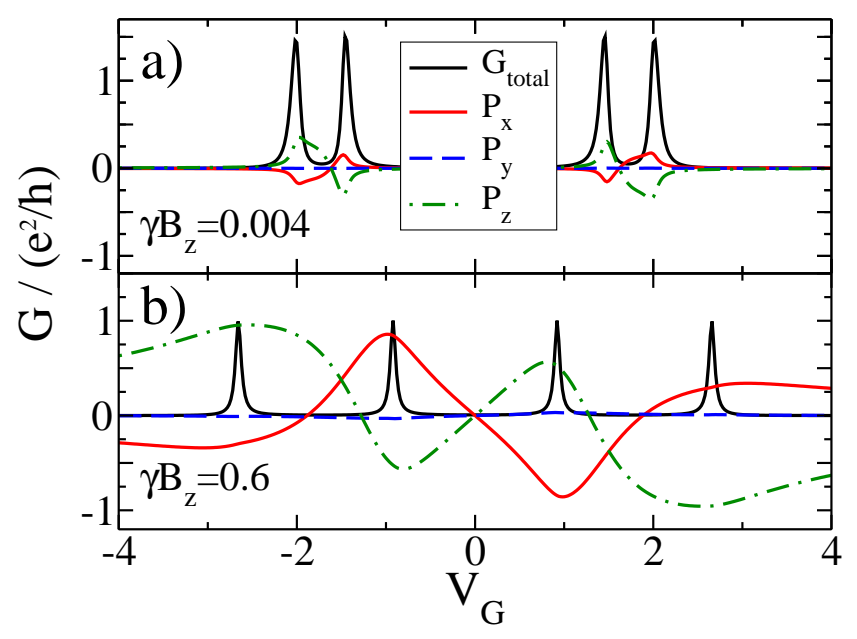

Figure 6.9: $G_{\text {total }}$ and $P_{i}$ of a double dot as a function of the gate voltage $V_{G}$ for Coulomb interaction $U_{1}=1, U_{2}=0.5$ and SOI $\alpha_{z}=0.5$.

a) For a small magnetic field, $\gamma B_{z}=0.004$, the spin polarization is negligible.

b) For larger fields, $\gamma B_{z}=0.6$, we almost obtain the same results as in the noninteracting case [see Fig. 6.4 a) ].

peaks, respectively. Since the peak separation is still quite small, the corresponding energy levels are close to each other. Therefore, the total conductance is not carried by a single conductance component as it is the case for larger splitting of the energy levels [see Sect.6.1.1]. Instead, further analysis reveals $G_{\uparrow \uparrow} / G_{\downarrow \downarrow} \sim 2$ for the left peak and accordingly $G_{\uparrow \uparrow} / G_{\downarrow \downarrow} \sim 0.5$ for the right peak within the double peak region for negative and positive $V_{G}$, respectively. Together with the degeneracy $G_{\uparrow \downarrow}=G_{\downarrow \uparrow}$, this leads to a fairly small polarization. The polarization would become even smaller (and finally zero) by further decreasing the magnetic field, i.e. allowing the system to reenter the Kondo regime.

The case of large magnetic fields, i.e. $\gamma B_{z}=0.6$, is studied in Fig. 6.9 b) and shows much larger spin polarization. Except for the finite Coulomb interaction, the parameters used here are the same as in Fig. 6.4 a). Apart from the altered peak position due to the renormalization of the system parameters, one obtains the same results as in the non-interacting case.

Thus, the Coulomb interaction does not seem to reveal any physical effects that could be helpful in this setup to increase the spin polarization or to create spinpolarized currents.

In the following, we will therefore study a modified setup with the double dot discussed in this section being positioned in one arm of an Aharonov-Bohm interferometer. 


\subsection{Aharonov-Bohm interferometer}

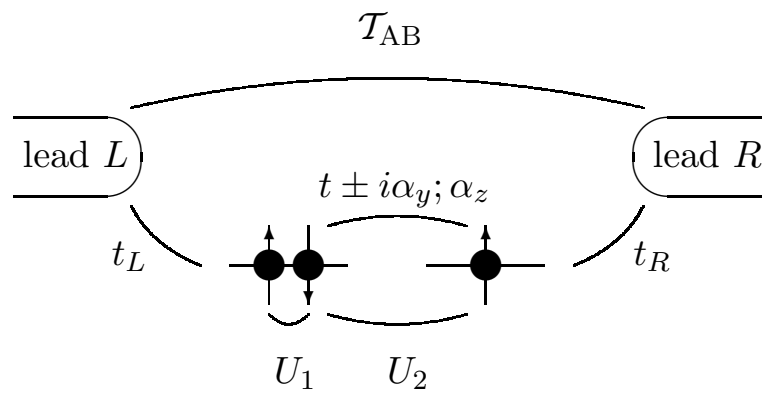

Figure 6.10: Geometry of an Aharonov-Bohm interferometer with a double quantum dot with SOI, magnetic field and Coulomb interaction in the lower arm.

The setup under consideration in this section is shown in Fig. 6.10. The interacting double quantum dot with magnetic field $\mathbf{B}=\left(0,0, B_{z}\right)$, conventional hopping $t$ and SOI hopping $\alpha_{y}$ and $\alpha_{z}$ is positioned in the lower arm of an Aharonov-Bohm interferometer (AB interferometer) and connected to the leads by hopping amplitudes $t_{L}$ and $t_{R}$ as before. The direct hopping from the left lead to the right lead via the upper arm of the interferometer is described by the complex $2 \times 2$-matrix $\mathcal{T}_{\mathrm{AB}}$. We allow for spin-conserving hopping $t_{\mathrm{SC}}$ and spin-flip hopping $t_{\mathrm{SF}}$ multiplied by spin-dependent phases $\exp \left( \pm i \phi_{\mathrm{SC}}\right)$ and $\exp \left(i \phi_{\mathrm{SF}}\right)$, respectively. Thus, $\mathcal{T}_{\mathrm{AB}}$ is given by

$$
\mathcal{T}_{\mathrm{AB}}=\left(\begin{array}{cc}
-t_{\mathrm{SC}} \exp \left(i \phi_{\mathrm{SC}}\right) & t_{\mathrm{SF}} \exp \left(i \phi_{\mathrm{SF}}\right) \\
-t_{\mathrm{SF}} \exp \left(i \phi_{\mathrm{SF}}\right) & -t_{\mathrm{SC}} \exp \left(-i \phi_{\mathrm{SC}}\right)
\end{array}\right)
$$

The hopping from the right lead to the left lead via the upper arm is given by $\mathcal{T}_{\mathrm{AB}}^{\dagger}$. One can think of $\phi_{\mathrm{SC}}$ and $\phi_{\mathrm{SF}}$ being tuned by a magnetic flux piercing the AharonovBohm setup in combination with SOI hopping in the upper arm. Whereas the magnetic flux leads to an overall spin-independent phase for all hopping components, it has been shown by Sun et al. in Ref. 74 for a slightly different setup with ferromagnetic leads that the SOI can lead to a spin-dependent phase. Considering only conventional hopping $t_{\mathrm{AB}}$ as well as SOI hopping $\alpha_{y ; \mathrm{AB}}$ and $\alpha_{z ; \mathrm{AB}}$ without a magnetic flux leads to $t_{\mathrm{SF}}=\alpha_{z ; \mathrm{AB}}, \phi_{\mathrm{SF}}=0$ and $t_{\mathrm{SC}}=\sqrt{t_{\mathrm{AB}}^{2}+\alpha_{y ; \mathrm{AB}}^{2}}, \phi_{\mathrm{SC}}=$ $\arccos \left(t / t_{\mathrm{SC}}\right)$ [and equivalently, $\phi_{\mathrm{SC}}=-\arcsin \left(\alpha_{y ; \mathrm{AB}} / t_{\mathrm{SC}}\right)$ ]. Although it might be difficult to tune $\phi_{\mathrm{SC}}$ and $\phi_{\mathrm{SF}}$ independently in experiments, we will consider the most general case and treat them as independent parameters in the following. 


\subsubsection{Non-interacting case}

Before we start analyzing the influence of Coulomb interaction induced correlation effects on the transport properties of the $\mathrm{AB}$ interferometer, we first focus on the non-interacting case to achieve an understanding of the interplay of the different system parameters.

\section{No SOI and no magnetic field in the lower arm}

In a first step, we concentrate on the simplest possibility with no SOI and no magnetic field being present in the lower arm of the $\mathrm{AB}$ interferometer.

Fig. 6.11 shows the total conductance $G_{\text {total }}$ of the non-interacing AharonovBohm interferometer as a function of the gate voltage $V_{G}$. In the lower arm, all parameters besides the hoppings $t=1$ and $t_{L}=t_{R}=0.2$ vanish. The small hybridization $\Gamma_{L}=\Gamma_{R}=0.04$ ensures that the double dot in the lower is weakly coupled to the leads such that its different energy levels are well separated. In Fig. 6.11 a), the spin-conserving hopping $t_{\mathrm{SC}}$ is varied for vanishing phase and vanishing spin-flip hopping, i.e. $\phi_{\mathrm{SC}}=0$ and $t_{\mathrm{SF}}=0$. For $t_{\mathrm{SC}}=0$, electrons can cross the $\mathrm{AB}$ interferometer only via the lower arm and one observes the same results as for the serial quantum dot, i.e. Lorentzian peaks at $V_{G}= \pm 1$.

For finite $t_{\mathrm{SC}}$, a complete suppression of $G_{\text {total }}$ is observed for a certain $V_{G}$. This phenomenon is known as the Fano effect and has been explained by U. Fano in 1961 (see Ref. 75]) as an interference effect of a discrete state with a continuum of states. Already in 1934, indication of this effect in a somewhat different system was observed experimentally by Whiddington and Priestley as doubly excited autoionizing resonances in the excitation spectrum of helium [76].

This Fano suppression of the conductance can be lifted by a finite phase $\phi_{\mathrm{SC}}$ as can be seen from Fig. 6.11 b). For $\phi_{\mathrm{SC}}=\pi / 2$, the conductance curve is perfectly symmetric w.r.t. $V_{G}=1$, i.e. more generally w.r.t. to the eigenenergy of the (separated) dot in the lower arm. One observes a $2 \pi$-periodicity of the $G_{\text {total }}$ with respect to $\phi_{S C}$ as expected from the structure of $\mathcal{T}_{\mathrm{AB}}$.

Fig. 6.11 c) shows $G_{\text {total }}$ for $t_{\mathrm{SF}}$ being changed at $\phi_{\mathrm{SF}}=0$ and $t_{\mathrm{SC}}=0$. Interestingly, no conductance suppression is observed for finite $t_{\mathrm{SF}}$, but rather an emerging Lorentzian peak at $V_{G}=1$ (the double dot's energy eigenvalue) from a finite ground level of the conductance at $V_{G}=0$ which increases with $t_{\mathrm{SF}}$. At $t_{\mathrm{SF}}=1$, one observes a perfect conductance, $G_{\text {total }}=2 e^{2} / h$, independent of $V_{G}$. This would also be expected, if there was not for a lower arm in the AB interferometer, but just a homogeneous one-dimensional wire without impurities. However, in our situation, we observe that the double dot is occupied by one electron at $V_{G}=1$ establishing transport through the lower arm. Thus, the interference of the two transport channels is perfectly constructive, but can be destroyed by a finite phase $\phi_{\mathrm{SF}}$.

Fig. 6.11 d) shows the influence of $\phi_{\mathrm{SF}}$ on the conductance for $t_{\mathrm{SF}}=0.5$. One observes a $\pi$-periodicity and pronounced minima close to the conductance peak for $\phi_{\mathrm{SF}}=\pi / 2$. Note, however, that these minima only occur, if $G_{\text {total }}\left(V_{G}=0\right) \geq 1$. For $G_{\text {total }}\left(V_{G}=0\right)<1$, simply the height of the conductance peak is decreased. 

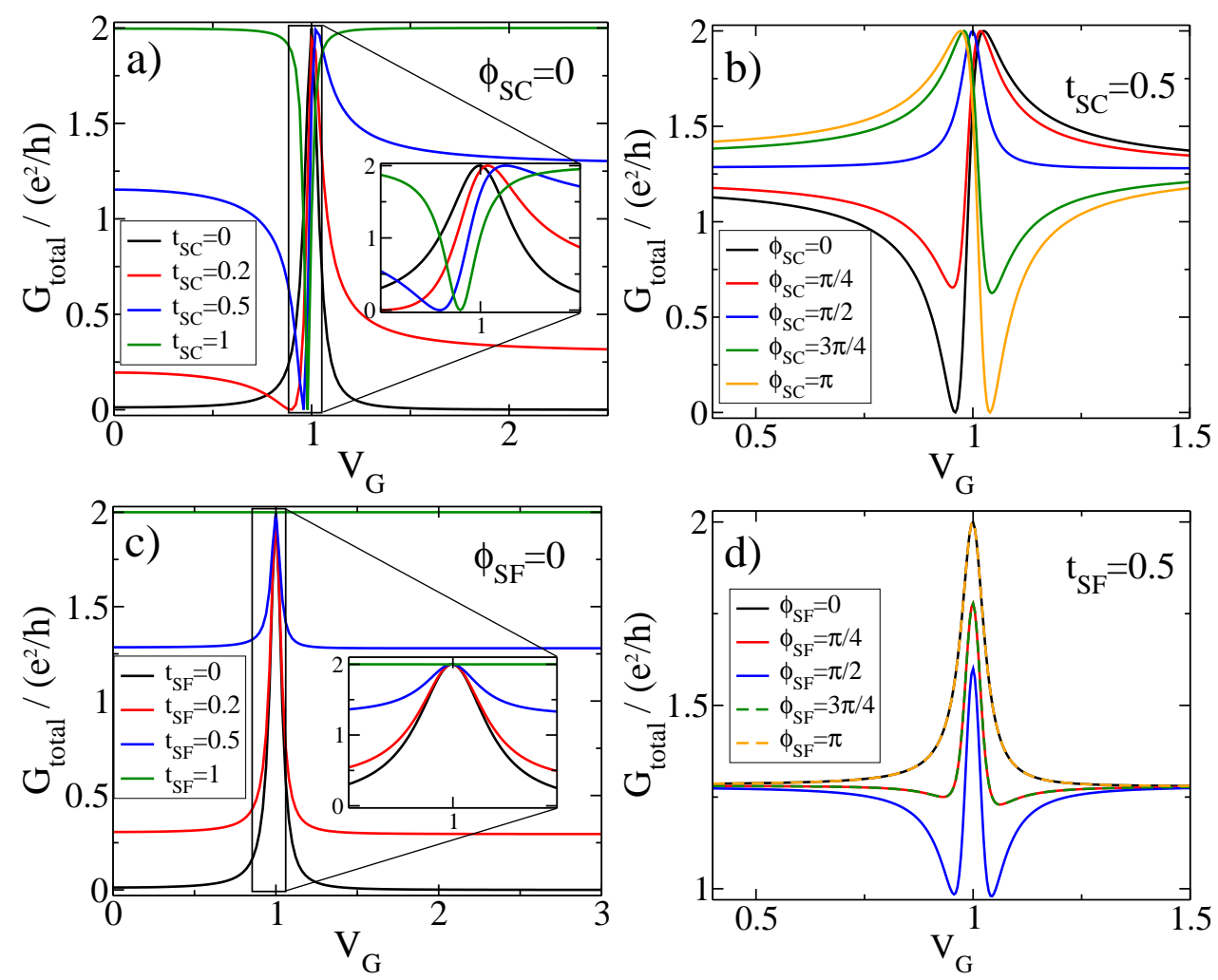

Figure 6.11: Total conductance $G_{\text {total }}$ of a non-interacting Aharonov-Bohm interferometer as a function of gate voltage $V_{G}$. Since the graphs are symmetric w.r.t. the axis $V_{G}=0$, only $V_{G} \geq 0$ is shown. The parameters of the lower arm are $t=1$ and $t_{L}=t_{R}=0.2$. All other parameters are zero.

a) The spin-conserving hopping in the upper arm is varied, $t_{S C}=0,0.2,0.5,1$ $\left(\phi_{\mathrm{SC}}=0\right)$, for vanishing spin-flip hopping $t_{\mathrm{SF}}$. The inset shows strongly pronounced Fano antiresonances.

b) For $t_{S C}=0.5$, the phase $\phi_{\mathrm{SC}}=0, \pi / 4, \pi / 2,3 \pi / 4, \pi$ is tuned. One observes a $2 \pi$-periodicity.

c) The spin-flip hopping in the upper arm is varied, $t_{S F}=0,0.2,0.5,1\left(\phi_{\mathrm{SF}}=0\right)$, for vanishing spin-conserving hopping $t_{\mathrm{SC}}$. The inset shows that there is no Fano effect.

d) For $t_{S F}=0.5$, the phase $\phi_{\mathrm{SF}}=0, \pi / 4, \pi / 2,3 \pi / 4, \pi$ is tuned. One observes a $\pi$-periodicity.

\section{SOI in the lower arm}

We now allow for finite SOI parameters $\alpha_{y}$ and $\alpha_{z}$ in the lower arm of the AB interferometer and study the interplay of $\alpha_{y}, \alpha_{z}$ and $t_{\mathrm{SC}}, t_{\mathrm{SF}}$. Figs. 6.12 a) and c) exhibit for $t_{\mathrm{SC}}=0.5\left(\phi_{\mathrm{SC}}=0\right)$ and $t_{\mathrm{SF}}=0$ that the Fano dip in the total conduc- 

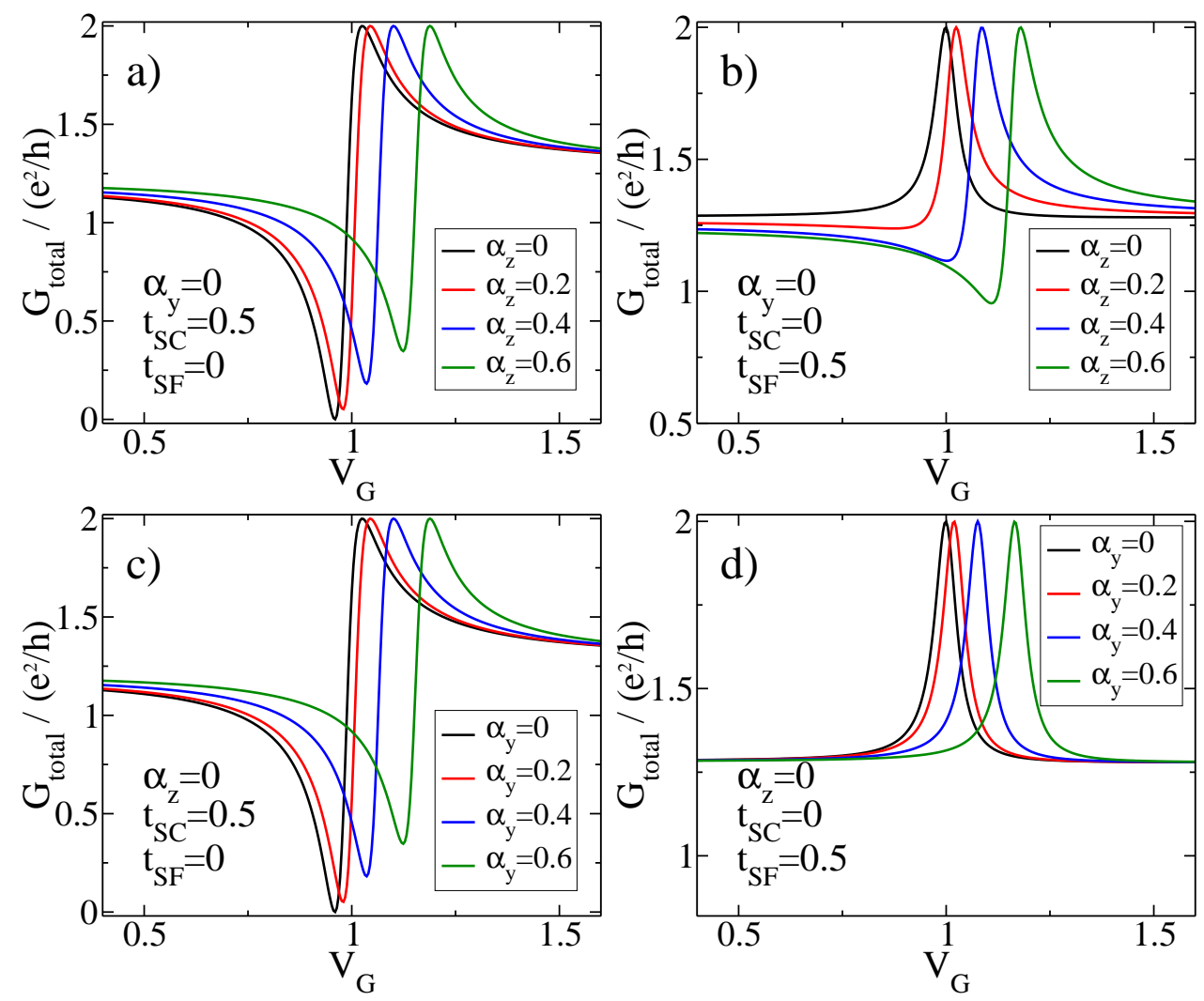

Figure 6.12: Influence of the SOI in the lower arm of an AB interferometer on the total conductance $G_{\text {total }}$ as a function of gate voltage $V_{G}$.

a) For $t_{\mathrm{SC}}=0.5\left(\phi_{\mathrm{SC}}=0\right)$ and $\alpha_{y}=t_{\mathrm{SF}}=0$, we diversify $\alpha_{z}$. The Fano dip at $\alpha_{z}=0$ is diminished with increasing $\alpha_{z}$.

b) For $t_{\mathrm{SF}}=0.5\left(\phi_{\mathrm{SF}}=0\right)$ and $\alpha_{y}=t_{\mathrm{SC}}=0$, a Fano dip evolves for finite $\alpha_{z}$ which is the more pronounced the larger $\alpha_{z}$.

c) For $t_{\mathrm{SC}}=0.5\left(\phi_{\mathrm{SC}}=0\right)$ and $\alpha_{z}=t_{\mathrm{SF}}=0$, a variation of $\alpha_{y}$ leads to exactly the same results as obtained in a) by varying $\alpha_{z}$.

d) For $t_{\mathrm{SF}}=0.5\left(\phi_{\mathrm{SF}}=0\right)$ and $\alpha_{z}=t_{\mathrm{SC}}=0$, a change of $\alpha_{y}$ just leads to a shift of the conductance peak to $\sqrt{t^{2}+\alpha_{y}^{2}}$.

tance $G_{\text {total }}$ is lifted, if either $\alpha_{z}$ or $\alpha_{y}$ is finite. Further investigation reveals that the conductance curves in this situation only depend on the effective SOI hopping $\alpha=\sqrt{\alpha_{y}^{2}+\alpha_{z}^{2}}$ and not on the ratio $\alpha_{y} / \alpha_{z}$. Since the situation with finite spinconserving hopping $t_{\mathrm{SC}}$, but vanishing spin-flip hopping $t_{\mathrm{SF}}$ in the upper arm could probably be realized in experiments, one can expect this effect to be measurable.

Setting the upper arm hopping parameters to $t_{\mathrm{SF}}=0.5\left(\phi_{\mathrm{SF}}=0\right)$ and $t_{\mathrm{SC}}=0$, the Lorentzian peak which is present in the case without SOI is transformed into 
a Fano-like resonance for finite $\alpha_{z}$ [see Fig. 6.12 b)]. The Fano dip is the more pronounced the larger $\alpha_{z}$. Indeed, for very large $\alpha_{z} \geq 3$, the conductance minimum approaches zero, but this situation will probably not be met in experiments [see Chapter 7 . Varying $\alpha_{y}$ at $\alpha_{z}=0$ merely leads to a shift of the conductance curve. The conductance peak is at $V_{G}=\sqrt{t^{2}+\alpha_{y}^{2}}$, but the shape of the curve remains unchanged [see Fig. 6.12 d)].

A Fano-like suppression of the conductance has also been observed by Sánchez et al. in Ref. 77] in linear ballistic quantum wires with local Rashba SOI. They considered several SOI subbands, thus establishing a discrete bound state. This state was coupled to the conduction states via Rashba intersubband mixing giving rise to similar interference effects as in our setup.

It seems quite astonishing that we do not find any spin polarization in all situations discussed so far, even if we tune the phases $t_{\mathrm{SC}}$ and $t_{\mathrm{SF}}$. However, as we have already seen for the serial dot, the SOI alone cannot lift the degeneracy of the spin-conserving and spin-flipping conductance components. Therefore, we allow for a finite magnetic field in the following.

\section{SOI and magnetic field in the lower arm}

As the tuning of the spin polarization is still one of our main goals, we apply an external magnetic field $\mathbf{B}=\left(0,0, B_{z}\right)$ to the double dot in the lower arm of the $\mathrm{AB}$ interferometer. Although this might lead to a magnetic flux through the setup in reality and would therefore influence the phases $t_{\mathrm{SC}}$ and $t_{\mathrm{SF}}$, we consider them to be independent variables in the following, since these parameters could also be realized by SOI in the upper arm as discussed earlier. Fig. 6.13 a) shows how the total conductance $G_{\text {total }}$ of the AB interferometer is influenced by a finite magnetic field $B_{z}$ for $t_{\mathrm{SC}}=0.5\left(\phi_{\mathrm{SC}}=0\right)$ and $\alpha_{y}=\alpha_{z}=t_{\mathrm{SF}}=0$. The typical Fano structure is doubled in a way that the two evolving Fano structures are separated by $\Delta V_{G}=2 \gamma B_{z}$. Whereas the amplitude of the Fano structure without magnetic field is $\Delta G_{\text {total }}=2 e^{2} / h$, i.e. allowing for perfect and totally suppressed conductance, the amplitude for finite magnetic field is in good approximation $\Delta G_{\text {total }}=1 e^{2} / h$. Setting $t_{\mathrm{SF}}=0.5\left(\phi_{\mathrm{SF}}=0\right)$ and $\alpha_{y}=\alpha_{z}=t_{\mathrm{SC}}=0$, one observes that the Lorentzian peak is transformed into two Lorentzian dips for finite magnetic field. The two dips are symmetric w.r.t. $V_{G}=1$ and are separated by $\Delta V_{G}=2 \gamma B_{z}$ [see Fig. 6.13 b)].

Tuning the SOI parameter $\alpha_{z}$ for fixed $t_{\mathrm{SC}}=0.5\left(\phi_{\mathrm{SC}}=0\right)$ and $\gamma B_{z}=0.2$ leads to a shift of the double Fano structure towards larger $V_{G}$ [see Fig. 6.13 c)]. The symmetry point is at $V_{G}=\sqrt{t^{2}+\alpha_{z}^{2}}$ and the distance between two corresponding minima (maxima) is reduced to $2 \gamma B_{z} / \sqrt{t^{2}+\alpha_{z}^{2}}$. Similarly to the case without any magnetic field [see Fig. 6.12 a) and c)], the total conductance depends only on $\alpha=\sqrt{\alpha_{y}^{2}+\alpha_{z}^{2}}$ and not on the ratio $\alpha_{y} / \alpha_{z}$ as long as $t_{\mathrm{SF}}=0$.

As can be seen from Fig. 6.13 d), increasing $\alpha_{z}$ for fixed $t_{\mathrm{SC}}=0.5\left(\phi_{\mathrm{SC}}=0\right)$ and $\gamma B_{z}=0.2$ leads to a formation of a Fano structure, which has already been observed for the case with vanishing magnetic field [see Fig. 6.12 b)]. Since there is 

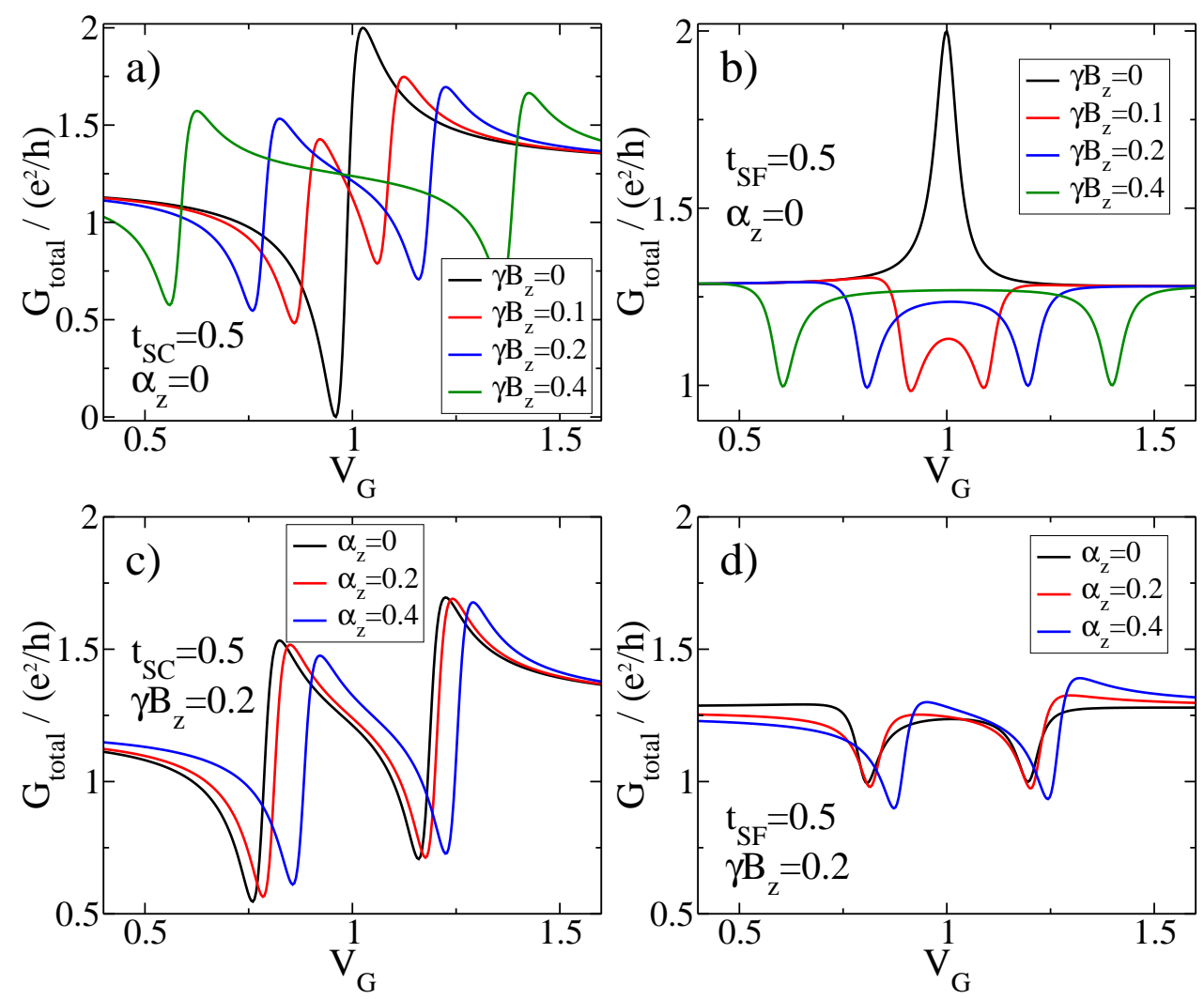

Figure 6.13: Influence of a magnetic field $B_{z}$ in the lower arm of an $\mathrm{AB}$ interferometer on the total conductance $G_{\text {total }}$ as a function of gate voltage $V_{G}$ in presence of SOI $\alpha_{z}\left(\alpha_{y}=0\right)$. The case with finite $\alpha_{y}$ is discussed in the text.

a) For $t_{\mathrm{SC}}=0.5\left(\phi_{\mathrm{SC}}=0\right)$ and $\alpha_{z}=t_{\mathrm{SF}}=0$, two Fano-like structures with smaller amplitude occur for finite magnetic field, which are separated by $2 \gamma B_{z}$.

b) For $t_{\mathrm{SF}}=0.5\left(\phi_{\mathrm{SC}}=0\right)$ and $\alpha_{z}=t_{\mathrm{SC}}=0$, the Lorentzian peak is transformed into two Lorentzian dips for finite magnetic field. The dips are separated by $2 \gamma B_{z}$ and the dip minimum is at $G_{\text {total }} \sim 1 e^{2} / h$, independent of $\gamma B_{z}$.

c) For $t_{\mathrm{SC}}=0.5\left(\phi_{\mathrm{SC}}=0\right), \gamma B_{z}=0.2$ and $t_{\mathrm{SF}}=0$, increasing $\alpha_{z}$ leads to a shift of the Fano double structure to larger $V_{G}$. The symmetry point is at $V_{G}=\sqrt{t^{2}+\alpha_{z}^{2}}$. d) For $t_{\mathrm{SF}}=0.5\left(\phi_{\mathrm{SC}}=0\right), \gamma B_{z}=0.2$ and $t_{\mathrm{SC}}=0$, two Fano-like structures evolve for increasing $\alpha_{z}$. The two minima are symmetric w.r.t. $V_{G}=\sqrt{t^{2}+\alpha_{z}^{2}}$.

a finite magnetic field present, two structures are observed. However, the sum of the amplitudes of the left and right structure is exactly the same as the amplitude of the single structure in the case without magnetic field for corresponding $\alpha_{z}$. Varying $\alpha_{y}$ in this situation only leads to a shift of the $(\alpha=0)$-curve. The analog symmetry point is at $V_{G}=\sqrt{t^{2}+\alpha_{y}^{2}}$. This influence of $\alpha_{y}$ for $t_{\mathrm{SF}} \neq 0$ and $t_{\mathrm{SC}}=0$ has also been observed in the case without magnetic field [see Fig. 6.12 d) for comparison]. 

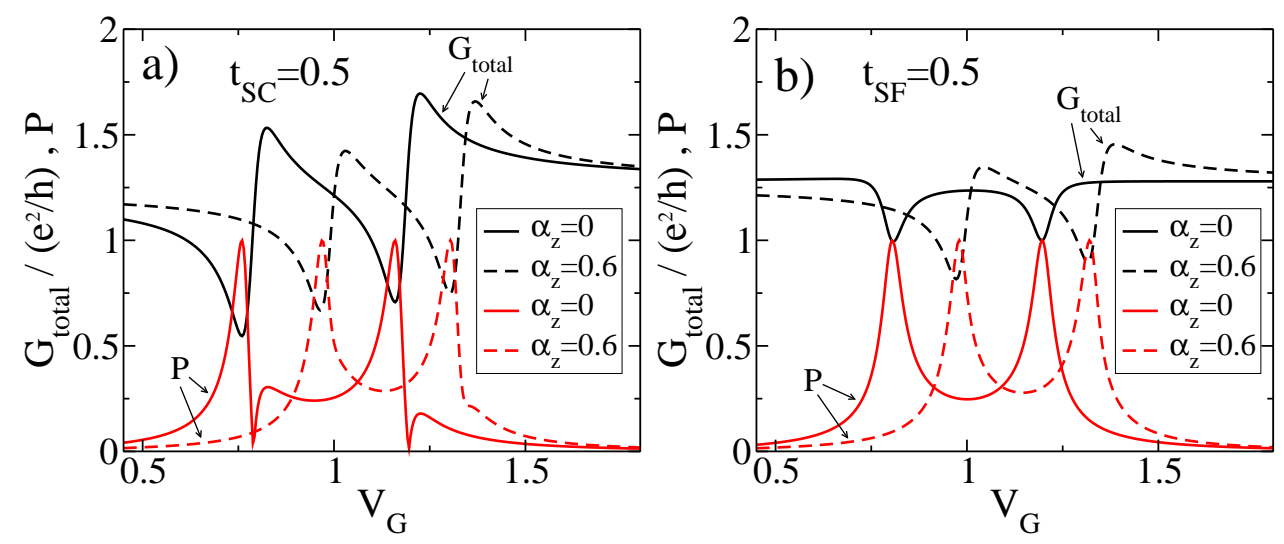

Figure 6.14: Effect of SOI on the spin polarization $P$ of a current flowing across the $\mathrm{AB}$ interferometer. The corresponding total conductance $G_{\text {total }}$ is also shown as reference.

a) For $t_{\mathrm{SC}}=0.5\left(\phi_{\mathrm{SC}}=0\right)$ and $t_{\mathrm{SF}}=0$, one observes two dips in the polarization with $P \rightarrow 0$ for $\alpha_{z}=0$ and two maxima with $P=1$. The dips vanish for finite $\alpha_{z}$ and the positions of the polarization maxima coincide with the positions of the minima of the corresponding conductance curve.

b) For $t_{\mathrm{SF}}=0.5\left(\phi_{\mathrm{SF}}=0\right)$ and $t_{\mathrm{SC}}=0$, the polarization reveals a double peak structure, both for $\alpha_{z}=0$ and $\alpha_{z}=0.6$. Finite SOI just leads to a shift of this structure towards larger $\left|V_{G}\right|$.

\section{Spin polarization}

Fig. 6.14 shows the influence of SOI on the polarization $P=\sqrt{P_{x}^{2}+P_{y}^{2}+P_{z}^{2}}$ of a current flowing across the $\mathrm{AB}$ interferometer. The individual components $P_{i}$ are not within our scope at the moment. $P$ is plotted over the gate voltage $V_{G}$ and the corresponding total conductance $G_{\text {total }}$ is also shown as a reference, since the application of spin polarization in terms of spintronic devices (spin transistor etc.) is favorable in regimes in which the total conductance is sizeable. As can be seen from Fig. 6.14 a) for the case with $t_{\mathrm{SC}}=0.5\left(\phi_{\mathrm{SC}}=0\right)$ and $t_{\mathrm{SF}}=0$, the spin polarization reveals maxima with $P=1$ at the same position as the conductance minima for $\alpha_{z}=0$ as well as for $\alpha_{z}=0.6$. The shift of the conductance curve for finite SOI has been discussed earlier, thus it is not remarkable that the polarization also shows this trend. The interesing feature is that the polarization dips with $P \rightarrow 0$ being present at the conductance maxima for $\alpha_{z}=0$ do not exist for finite $\alpha_{z}$. This cannot be explained by the increased effective hopping in the lower arm, but is more likely due to the interplay of the different phases of $\alpha_{z}, t$ and $t_{\mathrm{SC}}$. Changing the phase $\phi_{\mathrm{SC}}$ for $\alpha_{z}=0$ namely does not lead to a lift of the polarization dips.

For only spin-flip hopping being present in the upper arm, i.e. $t_{\mathrm{SF}}=0.5\left(\phi_{\mathrm{SF}}=0\right)$ and $t_{\mathrm{SC}}=0$ [see Fig. 6.14 $\mathrm{b}$ )], the spin polarization curve is just horizontally shifted 

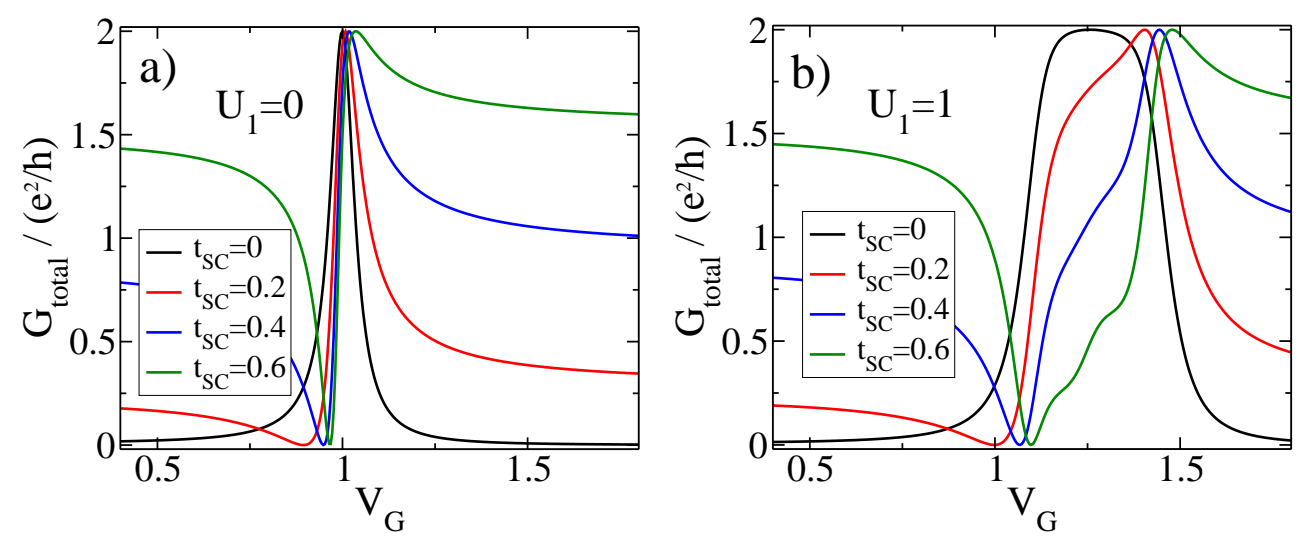

Figure 6.15: Influence of the on-site Coulomb interaction $U_{1}$ on $G_{\text {total }}$ as a function of $V_{G}$.

a) For $U_{1}=0$, increasing $t_{\mathrm{SC}}$ leads to a larger asymptotic value of $G_{\text {total }}$. The position of the Fano maximum/minimum only shows a sparse dependence on $t_{\mathrm{SC}}$.

b) For $U_{1}=1$, the asymptotic values of $G_{\text {total }}$ in good approximation remains the same compared to a), but in the Kondo region (for $t_{\mathrm{SC}}$ ) a combined structure of Kondo and Fano effect evolves for $t_{\mathrm{SC}}>0$.

by $\Delta V_{G}=\sqrt{t^{2}+\alpha_{z}^{2}}-t$ for $\alpha_{z}=0.6$ (taking the $\left(\alpha_{z}=0\right)$-curve as reference), but the shape remains exactly the same. Again, the position of the polarization maxima corresponds to the position of the conductance minima for both $\alpha_{z}=0$ and $\alpha_{z}=0.6$, but one does not observe any polarization dips.

We have only considered the total polarization so far. For real spintronic applications, one would naturally have to account for the direction of the spin polarization as well. Furthermore, tuning the phases $\phi_{\mathrm{SC}}$ and $\phi_{\mathrm{SF}}$ while varying the ratio $t_{\mathrm{SC}} / t_{\mathrm{SF}}$ might also lead to some interesting interference phenomena which should be reflected in the conductance and the spin polarization.

\subsubsection{Coulomb interaction}

We now include the Coulomb interaction via an on-site interaction $U_{1}$ and a nearestneighbor interaction $U_{2}$ [see Fig.6.10. The total conductance was shown in Sect.6.1.2 to exhibit Kondo plateaus in presence of Coulomb interaction. We now investigate how the Kondo effect interacts with the Fano effect in the present setup.

Fig. 6.15 b) shows the total conductance as a function of the gate voltage for constant $U_{1}=1\left(U_{2}=0\right)$. The hopping parameters in the lower arm are $t=1$ and $t_{L}=t_{R}=0.2$ and we tune $t_{\mathrm{SC}}$ in the upper arm. The Kondo plateau which is observed for $t_{\mathrm{SC}}=0$ is destroyed for $t_{\mathrm{SC}}>0$, but the gate voltage interval between the conductance minimum and the conductance maximum is of the width of the Kondo plateau meaning that the Kondo temperature still is the important energy scale of our system. The conductance within this interval is decreased with 

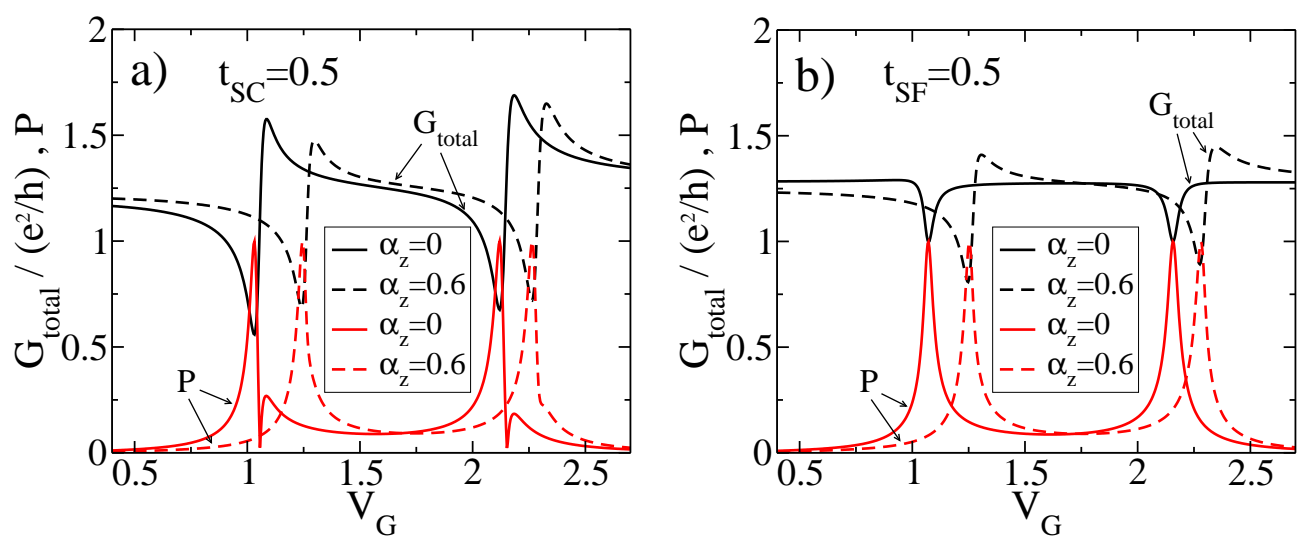

Figure 6.16: Influence of the SOI on the polarization and total conductance for finite Coulomb interaction $U_{1}=1$ and $U_{2}=0.5$.

a) For $t_{\mathrm{SC}}=0.5\left(\phi_{\mathrm{SC}}=0\right)$ and $t_{\mathrm{SF}}=0$, the polarization $P$ vanishes at the position of the conductance peaks for $\alpha_{z}=0$. These dips disappear for finite $\alpha_{z}=0.6$. In comparision to the non-interacting case [see Fig. 6.14 a)], the separation of the two polarization maxima is enlarged due to the increased renormalized magnetic field.

b) For $t_{\mathrm{SF}}=0.5\left(\phi_{\mathrm{SF}}=0\right)$ and $t_{\mathrm{SC}}=0, P$ and $G_{\text {total }}$ qualitatively reveal the same behavior as in the non-interacting case, both for $\alpha_{z}=0$ and $\alpha_{z}=0.6$. Again, the finite Coulomb interaction causes an enlargement of the polarization peak separation.

increasing $t_{\mathrm{SC}}$. The wave-like shape of the conductance curve for $t_{\mathrm{SC}}=0.6$ seems to be a numerical artifact at first sight. However, the vertex functions within the fRG flow do not become too large, which is an indication for a good numerical quality of the results. As the wave-structure reveals two inflection points, it might also come from the fact that a double dot is included in the lower arm of the $\mathrm{AB}$ interferometer. Anyway, the qualitative observation of the interplay of the Kondo and the Fano effect is not affected by this.

For $V_{G}$ outside the Kondo region, $G_{\text {total }}$ tends towards the non-interacting conductance [see Fig. 6.15 a)]. This means that transport is mainly conducted via the upper arm in this regime and explains the strong dependence of the asymptotic conductance on $t_{\mathrm{SC}}$.

Fig. 6.16 a) shows the total conductance and spin polarization for finite Coulomb interaction $U_{1}=1$ and $U_{2}=0.5$ and $t_{\mathrm{SC}}=0.5\left(\phi_{\mathrm{SC}}=0\right)$ and $t_{\mathrm{SF}}=0$. Again, the hopping parameters of the lower arm are $t=1$ and $t_{L}=t_{R}=0.2$. The two characteristic dips with vanishing polarization for $\alpha_{z}=0$ are lifted for finite SOI $\alpha_{z}=0.6$. This effect has already been observed in the case without Coulomb interaction [see Fig. 6.14 a)]. Comparing Fig. 6.16 b), where the same parameters have been used as in a), except for $t_{\mathrm{SF}}=0.5\left(\phi_{\mathrm{SF}}=0\right)$ and $t_{\mathrm{SC}}=0$, with the noninteracting equivalent [see Fig. 6.14 b)], also does not reveal any new features which 
could be traced back to collective behavior induced by the Coulomb interaction.

Overall, Fig. 6.16] indicates that there are only minor quantitativ changes due to the Coulomb interaction in the chosen parameter regime. The separation of the polarization maxima is increased due to the increased Zeeman splitting caused by the renormalized magnetic field. For gate voltages outside this characteristic structure, the values of total conductance and spin polarization correspond in good approximation to those obtained in the non-interacing case.

\subsection{Summary}

In this chapter, we have first studied the spin-dependent transport through a serial quantum dot described by a tight-binding model with two lattice sites connected to two semi-infinite Fermi liquid leads. As the SOI is reflected in spin-flip hopping and complex spin-conserving hopping, a double dot is the smallest system which can account for this effect.

The linear conductance as a function of an applied gate voltage in the noninteracting case revealed Lorentzian peaks the width of which is given by the hybridization $\Gamma=t_{L}^{2}+t_{R}^{2}$ of the quantum dot and the semi-infinite leads and which occurred whenever the dot's occupation number was changed. The peak position was predetermined by the energy eigenvalues of the decoupled quantum dot. In the lattice-spin base, the double dot's eigenvalue problem could be solved even in presence of SOI $\alpha_{y}$ and $\alpha_{z}$ and an external magnetic field $B_{z}$. It was shown that the SOI does not lift the spin degeneracy of the eigenvalues, but only causes a shift of the energy levels from $\pm t$ towards $\pm \sqrt{\alpha_{y}^{2}+\alpha_{z}^{2}+t^{2}}$, i.e. the SOI could be absorbed into an effective hopping for vanishing magnetic field.

For finite magnetic field, however, the spin degeneracy of the dot's eigenvalues was shown to be lifted due to the Zeeman splitting and the ratio $\alpha_{y} / \alpha_{z}$ gained importance, especially with respect to the direction of the spin polarization which revealed absolute values of $P=1$, i.e. perfect polarization, at the conductance peaks as long as the peaks were well-separated. Spin polarization could not be achieved just by tuning the SOI parameters, but a finite magnetic proved itself to be neccessary.

The Coulomb interaction was modeled by an on-site contribution $U_{1}$ and a nearest-neighbor contribution $U_{2}$ and led to a broadening of the conductance peaks, the Kondo effect. The Kondo effect arose to be inured to the SOI, as the timeinversion symmetry is not affected by the SOI, but could be destroyed by an external magnetic field which caused a conductance dip within the plateau region in the small field regime. For larger magnetic fields, the dip was broadened and the two resulting conductance maxima were separated by $2 \gamma B_{z}$. We identified the strength of the magnetic field which caused a dip down to $G_{\text {total }}=1 e^{2} / h$ with the Kondo temperature $T_{K}$, i.e. the energy scale relevant for the low-energy physics of our system. $T_{K}$ was determined as a function of all the system parameters and showed an exponential decreasing dependence on $U_{1}$ and $U_{2}$ (with slightly different prefactors) 
and a quadratic dependence on $\alpha$.

In the second part of this chapter, we studied the transport properties of an Aharonov-Bohm interferometer with the interacting double dot in one arm coupled to the reservoirs by $t_{L}$ and $t_{R}$ and a spin-dependent hopping channel in the other arm. The phase of the spin-dependent hopping could be thought of being tuned by a magnetic flux piercing the $\mathrm{AB}$ ring or otherwise being altered by allowing for SOI induced spin-flip and complex spin-conserving hopping parameters.

We observed Fano antiresonances leading to a vanishing conductance at a certain gate voltage. This dip in the conductance was scaled down with increasing SOI strength such that even for small SOI parameters a finite conductance was obtained at the position of the Fano antiresonance. On the other hand, a Fano-like structure could be generated from a Lorentzian structure by allowing for spin-flip hopping in the upper arm and a finite $\alpha_{z}$ in the lower arm. This was not observed for finite $\alpha_{y}$ in the lower arm indicating that $\alpha_{y}$ and $\alpha_{z}$ play entirely different roles in this setup. However, assuming the upper channel being governed by spin-conserving hopping alone, $\alpha_{y}$ and $\alpha_{z}$ led to the same results.

A finite magnetic field in the lower arm led to a split of the Fano structure and made a significant spin polarization possible. The maxima of the spin polarization conincided with the Fano dips of the total conductance independent of the SOI. For vanishing SOI, however, dips occured in the polarization, $P=0$, if transport via the upper arm was only possible by spin-conserving hopping. This feature was abandoned by a finite SOI.

Allowing for finite Coulomb interaction in the lower arm, the Fano structure was broadened to an interval of the Kondo plateau width. For a sizeable magnetic field, however, the total conductance and spin polarization revealed the same behavior as in the non-interacting case. We only observed minor quantitative modifications due to the renormalization of the system parameters.

In the final chapter of this thesis, we will give a short overview of the experimental situation regarding quantum dots and quantum wires. Based on results of ab initio calculations and experimental measurements, we will give a rough estimation of the magnitude of the parameters used in our models and discuss the experimental feasibility of building nanoscale devices which allow for spin polarization. 


\section{Chapter 7}

\section{Towards experimental realization}

The experimental realization of quantum dots and quantum wires has gained much attention in the past decade. Filed growth techniques of semiconductor heterojunctions as well as the implementation of metallic electrodes on top of these junctions allow for confining electrons to a very small spatial area which in good approximation can be seen as a zero- or one-dimensional electron system.

\subsection{Realization of quantum dots}

Quantum dots are usually implemented by confining electrons in a 2DEG with an array of metallic gate electrodes. GaAs/AlGaAs heterojunctions are often used to establish the 2DEG with a thin AlGaAs layer of $\sim 100 \mathrm{~nm}$ being grown on the GaAs substrate using molecular beam epitaxy. The electrodes are mounted on top of the AlGaAs layer using e.g. photolithography.

Fig. 7.1 shows a scanning electron microscope (SEM) picture of such a GaAs double quantum dot. The metallic gates $\mathrm{L}$ and $\mathrm{R}$ define the double dot in the 2DEG about $100 \mathrm{~nm}$ below the surface by depletion of this area, ideally allowing for single electron transport. The tunnel barrier between the left dot and the right dot can be tuned via the upper electrode in the middle. Applying a gate voltage to the lower three electrodes changes the dot's energy. (This gate voltage is exactly the parameter we used in Chapter [6] to modify the double dot's transport properties.) The source and drain contacts are not shown here. Quantum point contacts (QPC) on the left and right side measure the conductances $g_{l s}$ and $g_{r s}$ which reflect the average occupation of the left/right dot. This setup has been used in Ref. [78] to study the triplet-singlet spin relaxation via the nuclei of the host atoms in presence of a magnetic field. It has also been shown in Ref. [79] that coherent manipulation of coupled electron spins can be achieved with this setup. The spin dephasing time usually limited to $\sim 10 \mathrm{~ns}$ by hyperfine interactions with the GaAs host nuclei could thereby be extended to a coherence time larger than $1 \mu \mathrm{s}$ for a two-electron spin state. 
Thus, one can think of these double dots being a first attempt towards realization of a spin qubit.

The Kondo effect has been observed for quantum dots in GaAs [68 as well as in InAs [72, both being materials which reveal a strong SOI. However, although this indicates that the Kondo effect is not destroyed by the SOI, its quantitative effect on the Kondo scale $T_{K}$ has not yet been investigated experimentally. Therefore, it is important to achieve control over the SOI strength by external electric fields. These fields can be implemented easily by additional gate electrodes, but the parameter range in which the SOI can be tuned without any unintentional side effects might be small.

The possibility of non-local spin control has been reported in Ref. [80 for a system consisting of a double quantum dot with the left dot and the right dot being coupled by an open conducting region. It was shown that Kondo resonances in one dot could be suppressed by changing the electron number and the coupling of the other dot. However, this effect could not be observed within our framework.

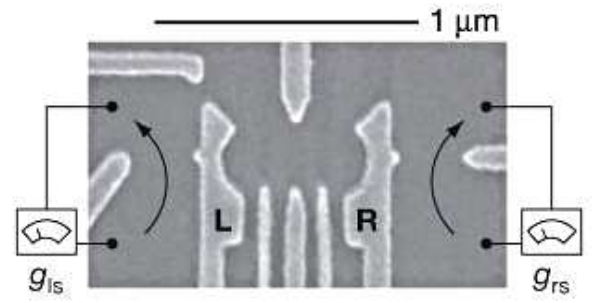

Figure 7.1: SEM picture of a GaAs double quantum dot in a 2DEG defined between two metallic gates $\mathrm{L}$ and $\mathrm{R}$. The tunnel barrier between the left and the right dot is tuned by a gate voltage applied to the upper electrode in the middle. The three lower electrodes are used to change the dot's energy. QPCs on the left and right measure the conductances $g_{l s}$ and $g_{r s}$ which reflect the average occupation of each dot. Reprinted by permission from Macmillan Publishers Ltd: Nature 435, 925, copyright 2005 .

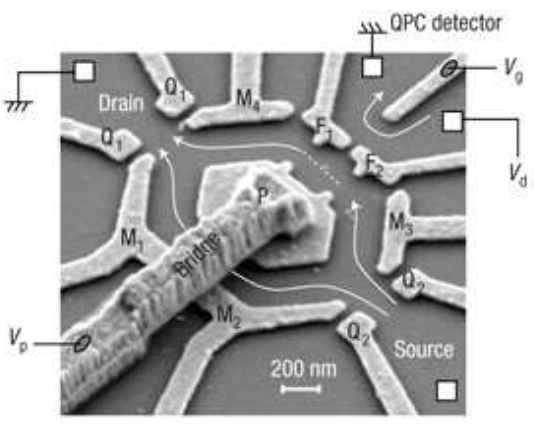

Figure 7.2: SEM picture of an Aharonov-Bohm ring in a 2DEG formed at a GaAs/AlGaAs heterojunction. The side gates $\mathrm{M}_{1}, \mathrm{M}_{2}, \mathrm{M}_{3}$ and $\mathrm{M}_{4}$ together with an island gate $\mathrm{P}$ define the $\mathrm{AB}$ ring. The source and drain contacts are shown. An additional quantum dot with its properties being controlled by the voltages $V_{g}$ and $V_{d}$ and a QPC detector are placed to detect charges trapped inside the upper interferometer arm. Reprinted by permission from Macmillan Publishers Ltd: Nature 4, 205, copyright 2008. 
A more subtle setup is depicted in Fig. 17.2, The SEM picture shows an AharonovBohm interferometer fabricated in the 2DEG formed at a GaAs/AlGaAs heterojunction. The $\mathrm{AB}$ ring is defined by the gates $\mathrm{M}_{1}, \mathrm{M}_{2}, \mathrm{M}_{3}$, and $\mathrm{M}_{4}$ together with an island gate $\mathrm{P}$. The lower arm can be pinched off by applying a large negative voltage to $\mathrm{M}_{1}$ and $\mathrm{M}_{2}$, thus allowing for a separate study of the transport properties of the quantum dot in the upper arm. The dot's occupation number is determined by measuring the conductance at the QPC detector. The tunnel barrier to the higherdimensional environment (the leads in our model) is tuned by the gates $\mathrm{Q}_{1}$ and $\mathrm{Q}_{2}$. This setup has been used in Ref. [81] to study Aharonov-Bohm oscillations and quantum interference.

\subsection{Realization of quantum wires}

Basically, three methods exist to experimentally realize quantum wires. The first is similar to the method described in the previous section. Two parallel gate electrodes on top of a semiconductor heterojunction can be used to confine the electrons in the 2DEG in a second direction leaving the possibility of electronic motion in one direction only. Experimentally, this might be the most difficult realization.

Another possibility is to use the cleaved-edge overgrowth technique [82, 83. Using this method, one cleaves a sample with a semiconductor heterojunction orthogonal to the junction twice and overgrows the cleavage planes with single quantum wells in order to confine the electrons to one dimension.

However, nowadays most attention is spent on carbon nanotubes as realization of quantum wires. They can be deposited by e.g. spin-coating on a semiconductor substrate and besides their outstanding mechanical properties like tensile strength and elastic modulus they also reveal excellent electronic properties. Coiled up from a flat sheet of graphene, the nanotube's conductance behavior strongly depends on the chiral vector, i.e. the coil direction, and can show semiconducting as well as metallic behavior.

Fig. 7.3 shows an atomic force microscope (AFM) picture of a single-wall carbon nanotube with $\mathrm{Pt}$ electrodes on top of a $\mathrm{Si} / \mathrm{SiO}_{2}$ substrate as used in Ref. [45. The electrode in the upper left corner is used to change the electrostatic potential of the nanotube via an applied gate voltage and a bias voltage is applied between the other two electrodes.

It has been pointed out in Ref. 84 that single-wall carbon nanotubes can be spin-polarized in low magnetic fields. Furthermore, a change of the electronic spectrum as well as induced spin-flips by changing the applied gate voltage have been reported giving rise to the assumption that electron-electron interactions play an important role. Recently, a sizeable spin-orbit interaction has been observed in carbon nanotubes by excited-state spectroscopy [22].

These observations make carbon nanotubes ideal candidates for experimental setups in which the spin-polarization effects described in this thesis could be measured. 


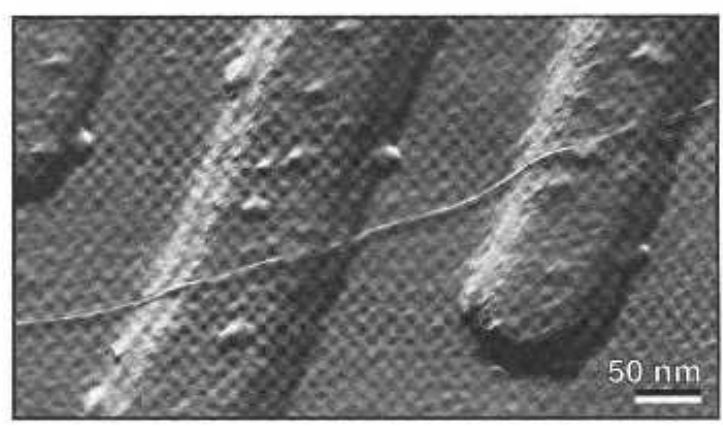

Figure 7.3: AFM picture of a carbon nanotube on top of a $\mathrm{Si} / \mathrm{SiO}_{2}$ substrate with $\mathrm{Pt}$ electrodes. The electrode in the upper left corner is used to change the electrostatic potential of the nanotube via an applied gate voltage. A bias voltage is applied between the other two electrodes. Reprinted by permission from Macmillan Publishers Ltd: Nature 386, 474, copyright 1997.

\subsection{The system parameters}

In general, lattice models are believed to be superior to continuum models in terms of adequate modeling microscopic solid state materials, especially in low dimensions. We therefore estimate the magnitude of the parameters used in our model in order to show the experimental feasibility.

A common guess for the conventional hopping matrix element is $t \sim 1 \mathrm{eV}$ [85]. Unfortunately, in the literature one finds huge deviations of the values for the Coulomb interaction matrix elements $U_{1}$ (local) and $U_{2}$ (nearest-neighbor), depending on the numerical method and asumptions made to the underlying physics, e.g. material under consideration and screening of the electron charge. Without screening effects, $U_{1} \sim 20-25 \mathrm{eV}$ and $U_{2} \sim 5 \mathrm{eV}$ have been obtained for $3 \mathrm{~d}$ transition metals using Wannier functions in a spherical-harmonics expansion [86]. It was pointed out in Ref. [86] that constrained local density approximation calculations, which include some screening effects, yield values of the same order for $U_{1}$ and $U_{2}$. However, $U_{1}=2-4 \mathrm{eV}$ has also been reported for the same group of materials using self-consistent Hartree-Fock approximation [87. For GaAs, which is a material much more common for quantum wires than a $3 \mathrm{~d}$ metal, $U_{1}=0.56 \mathrm{eV}$ has been obtained by density functional theory [88. Thus, we believe that the values for $U_{1}$ and $U_{2}$ chosen in this thesis should at least roughly give a good estimate.

The strength of the Rashba spin-orbit field is believed to be in the range of $0.5-4 \times 10^{-11} \mathrm{eVm}$ for InAs with theoretical and experimental values corresponding quite well [89]. This would lead to a spin-orbit coupling parameter $\alpha \sim 0.1 \mathrm{eV}$ for our lattice model, assuming a lattice spacing of several angstrom. The values reported in Ref. [89] refer to the bulk of InAs and not a heterostructure interface, 
which is necessary to obtain a well defined quantum wire. One can assume that the electric field is enlarged at such an interface leading to a somewhat larger spin-orbit coupling. Moreover, it has been shown experimentally that the SOI strength in semiconductor heterostructures can be tuned by applying a gate voltage [90, 91, 92 . In our calculations, we chose systems consisting of $N \sim 100-5000$ lattice sites. This corresponds to an overall length of $50-2500 \mathrm{~nm}$ for a lattice spacing of half a nanometer, which should be a realistic guess and also feasible in experiments.

Thus, all system parameters assumed in this thesis should at least roughly be achievable in experimental setups. Furthermore, we believe that attaching a 1D quantum wire to higher-dimensional leads which can be regarded as Fermi liquids is a suitable approach to model the experimental situation. 


\section{Chapter 8}

\section{Conclusions and outlook}

In this thesis, we investigated the influence of the (Rashba) spin-orbit interaction (SOI) on the transport properties of correlated electrons in quantum wires and quantum dots and analyzed in which parameter regimes spin polarization can be obtained.

In the first part, we neglected the Coulomb interaction and started with the derivation of the SOI Hamiltonian in the continuum form as a relativistic effect following directly from the Dirac equation in second order $\mathcal{O}\left(v^{2} / c^{2}\right)$. We then confined the system to one dimension showing that the SOI plays a role, whenever the electrons are confined by an external scalar potential with large gradients. For a strictly one-dimensional confinement, the scalar potential had to be chosen in a way that two SOI parameters $\alpha_{y}$ and $\alpha_{z}$ emerged (for transport in $x$-direction). We also allowed for a magnetic field in arbitrary direction being represented by the Zeeman Hamiltonian and solved the corresponding eigenvalue problem. The interplay of the SOI leading to a splitting of the quadratic energy dispersion $\epsilon^{(s)}(k)$ in $k$-direction (with $s= \pm$ labeling the different dispersion branches) with the magnetic field inducing a Zeeman gap was discussed.

Aiming for control over the spin polarization in terms of spin expectation values, we expanded the setup originally proposed by Strěda \& Sěba in Ref. [26] and addressed the scattering problem at a potential step and a potential barrier for the special case of the magnetic field being parallel to the quantum wire in presence of the two SOI parameters $\alpha_{y}$ and $\alpha_{z}$. A sizeable spin polarization was shown to be feasible and controllable by tuning the energy of the incoming electrons within a suitable parameter regime for energies close to the gap of the energy dispersion. The SOI was shown to play a crucial role regarding the size and the direction of the spin polarization. The ratio of the SOI parameters was shown to determine the direction of the polarization orthogonal to the wire via $\left|\alpha_{y} / \alpha_{z}\right|=\left|P_{z} / P_{y}\right|$, whereas the total polarization was determined by the SOI strength $\alpha=\sqrt{\alpha_{y}^{2}+\alpha_{z}^{2}}$.

In Chapter 3. we constructed a lattice model in terms of localized Wannier states which exhibited the same low-energy properties as the continuum model in terms of energy dispersion and spin expectation values. We confined the lattice model to 
a finite size of $N$ lattice sites and attached it to two semi-infinite leads via hopping amplitudes $t_{L, R}$ thereby matching a situation which is far more realistic than the infinite continuum model. Projecting out the leads by a Feshbach projection, we were left with a finite scattering problem for $2 N \times 2 N$-matrices which yielded the propagator $\mathcal{G}$.

The linear transport through the system was studied within the Landauer-Büttiker formalism (see e.g. Ref. [41]). We calculated the linear conductance $G_{\sigma \sigma^{\prime}}$ from the transmission $\mathcal{T}_{\sigma \sigma^{\prime}}$ both of which were shown to exhibit two spin indices $\sigma, \sigma^{\prime} \in\{\uparrow, \downarrow\}$ (four conductance components) in our case with SOI the first index labeling the (z-component of the) spin of the (incoming) electron in the left lead and the second index labeling the (z-component of the) spin of the (outgoing) electron in the right lead. Using the Kubo formula (see e.g. Ref. [42]), we obtained $\mathcal{T}_{\sigma \sigma^{\prime}}$ from the $(1, N)$-matrix element $\mathcal{G}_{\sigma \sigma^{\prime}}(1, N)$ of the propagator.

Studying the linear conductance as a function of the chemical potential $\mu$ of the entire system (quantum wire and leads), we matched a situation analog to altering the Fermi energy of the incoming energies in the continuum case. Due to the finite band width of the leads, however, the chemical potential was restricted to $\mu \in(-2, \ldots, 2)$ (in units of the lead hopping). In order to avoid backscattering into the leads, we adiabatically turned on/off all system parameters (like SOI and magnetic field) which were not present in the leads and obtained a perfect total conductance (being the sum of the four components) $G_{\text {total }}=2 e^{2} / h$. We neglected any SOI and magnetic field in the leads, which would make the measurement of spin polarization difficult, as the spin would be no conserved quantitiy in the leads. However, a finite SOI in the leads could be absorbed into an effective lead hopping $t_{\text {eff }}=\sqrt{t^{2}+\alpha_{y}^{2}+\alpha_{z}^{2}}$ and the same was shown to hold for the contacts between the leads and the quantum wire.

Similar to the Datta \& Das-transistor, we observed an oscillatory behavior of the conductance components with the system length $N$ with an oscillation period being inverse proportional to the SOI strength, $N_{\text {osc }} \sim 1 / \alpha$ with $\alpha=\sqrt{\alpha_{y}^{2}+\alpha_{z}^{2}}$. The spinconserving and spin-flip components were degenerate, respectively, i.e. $G_{\uparrow \uparrow}=G_{\downarrow \downarrow}$ and $G_{\uparrow \downarrow}=G_{\uparrow \downarrow}$. In presence of a magnetic field $\gamma B_{x}$ (in $x$-direction), we obtained an energy range with two open conducting channels and, consequently, $G_{\text {total }}=$ $2 e^{2} / h$ for $\mu$ being in the energetic bulk of the leads and two ranges of width $\gamma B_{x}$ at the band edges with one conducting channel and $G_{\text {total }}=1 e^{2} / h$. This was identified as a trivial band effect due to the Zeeman splitting. The components $G_{\sigma \sigma^{\prime}}$ revealed a strong oscillatory behavior inside the range with two conducting channels, which could be altered by the SOI. Even in presence of SOI the above-mentioned degeneracy of the components was conserved. Outside this range, this degeneracy was lifted and no significant oscillations could be observed.

For a finite potential step $V_{0}$ in the middle of the quantum wire, we discovered an energy range of width $V_{0}$ (just below the range with two conducting channels) within which a sizeable spin polarization could be observed which was shown to react very sensitively on the chemical potential $\mu$. Increasing $V_{0}$ beyond a critical value led to 
a vanishing linear conductance which was explained within a single-particle picture as an energetical rupture of the bands.

Furthermore, we studied the linear conductance and spin polarization in presence of a single impurity $V_{1}$ representing the potential barrier discussed in the continuum case. Whereas $G_{\text {total }}$ was discreased with $V_{1}$ and the step-like shape of the ranges with one and two conducting channels was washed out, the spin polarization could be tuned over a wide range by setting the SOI and the magnetic field accordingly and also showed a strong dependence on $V_{1}$.

As conventional methods like first-order perturbation theory and self-consistent Hartree-Fock approximation are known to break down in low-dimensional systems with Coulomb interaction, we gave a short introduction to the functional renormalization group method (fRG) in Chapter 4. Starting with the generating functional for the $m$-particle vertex functions, we introduced an infrared cutoff $\Lambda$ to the free propagator, $\mathcal{G}^{0} \rightarrow \mathcal{G}^{0, \Lambda}$. Derivation of this functional w.r.t. $\Lambda$ led to an exact hierarchy of flow equations for the vertex functions. This hierarchy had to be truncated in order to integrate the remaining flow equations from a large starting value $\Lambda_{0}$ down to $\Lambda=0$. At the end of the fRG flow, we were left with an effective one-particle problem with the renormalization of the system parameters being included in the system's self energy $\Sigma^{\Lambda=0}$.

In Chapter [5, we included the Coulomb interaction into our model via a local part $U_{1}$ and a nearest-neighbor part $U_{2}$. Using the fRG method, we first studied short quantum wires in relation to which the flow of the two-particle vertex could be neglected. Due to the renormalization of the system parameters, the linear conductance and the spin polarization were altered in our systems with potential step or single impurity, but the qualitative results remained the same.

Thereafter, we studied long quantum wires with single impurities which are known to reveal characteristics of inhomogeneous Luttinger liquids like power-law scaling of correlation functions like the linear conductance (see e.g. Ref. [57]). For given $\mu$, we chose the ratio $U_{1} / U_{2}=-2 \cos \left(2 k_{F}\right)=2-\mu^{2}$ in order to tune the backscattering component $g_{1, \perp}$ to zero which, again, allowed us to neglect the flow of the two-particle vertex. We calculated the linear conductance as a function of the system size $N$ with $\delta=v_{F} / N$ being the relevant energy scale for the low-energy excitations and obtained the expected power laws. In presence of SOI, we obtained the same exponents as those predicted in leading order [59, 63 by replacing the conventional hopping $t$ with an effective hopping $t_{\text {eff }}=\sqrt{t^{2}+\alpha_{y}^{2}+\alpha_{z}^{2}}$. However, the spin polarization did not reveal any power law behavior, but showed oscillations with constant frequency and amplitude as a function of the system size.

Including a potential step $V_{0}$ into the system caused a step in the profile of the particle density $\left\langle n_{j}\right\rangle$. Although power laws could be extracted from the spectral function on each side of the step, the exponents did not correspond to the respective filling, even when we drove the system into the asymptotic limit by an additional strong single impurity $V_{1}$.

In Chapter 6] we studied the transport properties of a double $\operatorname{dot}(N=2$ lattice 
sites) in presence of SOI and a magnetic field. As before, the dot was attached to two non-interacting leads without SOI and magnetic field. For the non-interacting case, all properties of the linear conductance could be explained by analyzing the eigenvalue problem of the decoupled dot. Since the quantum dot was much easier to handle numerically than the quantum wires, we were able to account for the flow of the two-particle vertex in this setup. We studied the linear conductance as a function of a gate voltage applied to the dot. Electron correlations led to the transformation of the Lorentzian conductance peaks into Kondo plateaus which were affected by the SOI just by the effective hopping $t_{\text {eff }}$, but did not change their shape. However, the Kondo plateaus were destroyed by an external magnetic field and we identified the magnetic field which led to a dip $G_{\text {total }}=1 e^{2} / h$ within the Kondo plateau region with the Kondo temperature $T_{K}$, i.e. the relevant energy scale in this situation. We observed an exponential dependence of $T_{K}$ on $U_{1}$ and $U_{2}$ with slightly different exponential prefactors. The prefactor for $U_{1}$ corresponded very well with the one predicted in first order [70], but no reference was found for the prefactor of $U_{2}$. Furthermore, $T_{K}$ revealed a quadratic dependence on the SOI strength $\alpha$.

In a next step, we included the double dot in one arm of an Aharonov-Bohm (AB) interferometer and studied spin-dependent interference effects and the interplay of the Kondo and the Fano effect. For certain parameter regimes, dips in the polarization could be lifted by a finite SOI on the quantum dot. In addition, interference effects revealed to stongly influence the total conductance.

Finally, in Chapter 7 we gave a short overview of experiments which have been performed on quantum dots and quantum wires showing that the setups discussed in this thesis might match realistic experimental situations and, therefore, the polarization effects obtained here might also be observable experimentally. Finally, we compared the dimension of the different system parameters used in this thesis with values found in the literature finding quite a good agreement.

The parametrization of the two-particle vertex in presence of SOI and the solution of the corresponding flow equations remains a major task for the future. Furthermore, subband mixing was neglected in our approach, but is known to reveal subtle effects on the spin polarization and the transport properties for finite SOI. Further structuring of quantum wires by potential steps and several single impurities would certainly lead to an interesting behavior of the linear conductance and the spin polarization. Last but not least, higher order SOI (following from an expansion of the Dirac equation in higher than second order) might also be taken into account. 


\section{Appendix A}

\section{The Green's function of the leads}

The derivation of the Green's function of the leads in presence of SOI follows the same idea as in the case without SOI (see Refs. [17, 40]). Consider a semi-infinite

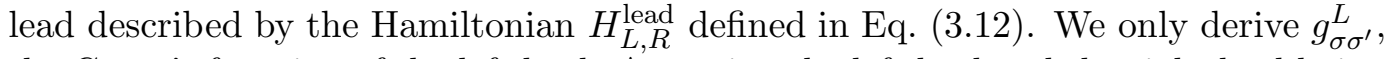
the Green's function of the left lead. Assuming the left lead and the right lead being identical, we obtain $g_{\sigma \sigma^{\prime}}^{R}=g_{\sigma \sigma^{\prime}}^{L}$.

The lattice site index runs from $-\infty$ up to some $j$ and we identify the Green's function at this rightmost site with the resolvent of the lead Hamiltonian, projected to this site, i.e. $g_{\sigma \sigma^{\prime}}(z)=\left(z-H_{L}^{\text {lead }}\right)_{\sigma \sigma^{\prime}}^{-1}(j ; j)$. We now add one lattice site $j+1$ with the same hopping parameters $t, \alpha_{y}$ and $\alpha_{z}$ to the right of our semi-infinite lead. Since the lead is semi-infinite and homogeneous the Green's function $\tilde{g}_{\sigma \sigma^{\prime}}(z)$ at the new site $j+1$ should be the same as $g_{\sigma \sigma^{\prime}}(z)$. In the same way as for the case without SOI (see Ref. [17] for details), we therefore obtain

$$
\begin{aligned}
\tilde{g}_{\sigma \sigma^{\prime}}^{-1}(z)= & \left(z-H_{L}^{\text {lead }}\right)_{\sigma \sigma^{\prime}}(j+1 ; j+1) \\
& -\sum_{\sigma^{\prime \prime}, \sigma^{\prime \prime \prime}}\left(H_{L}^{\text {lead }}\right)_{\sigma \sigma^{\prime \prime}}(j+1 ; j)\left(z-H_{L}^{\text {lead }}\right)_{\sigma^{\prime \prime} \sigma^{\prime \prime \prime}}^{-1}(j ; j)\left(H_{L}^{\text {lead }}\right)_{\sigma^{\prime \prime \prime} \sigma^{\prime}}(j ; j+1) \\
= & (z+\mu) \delta_{\sigma \sigma^{\prime}} \\
& -\sum_{\sigma^{\prime \prime}, \sigma^{\prime \prime \prime}}\left\{\left[-t \delta_{\sigma \sigma^{\prime \prime}}-\alpha_{z} \delta_{\sigma-\sigma^{\prime \prime}}\left(\delta_{\sigma \uparrow}-\delta_{\sigma \downarrow}\right)+i \alpha_{y} \delta_{\sigma \sigma^{\prime \prime}}\left(\delta_{\sigma \uparrow}-\delta_{\sigma \downarrow}\right)\right] g_{\sigma^{\prime \prime} \sigma^{\prime \prime \prime}}(z)\right. \\
& \left.\times\left(-t \delta_{\sigma \sigma^{\prime \prime}}-\alpha_{z} \delta_{\sigma-\sigma^{\prime \prime}}\left(\delta_{\sigma \uparrow}-\delta_{\sigma \downarrow}\right)+i \alpha_{y} \delta_{\sigma \sigma^{\prime \prime}}\left(\delta_{\sigma \uparrow}-\delta_{\sigma \downarrow}\right)\right]\right\} \\
& \stackrel{!}{=} g_{\sigma \sigma^{\prime}}^{-1}(z)
\end{aligned}
$$

also allowing for a chemical potential $\mu$ in the leads. For convenience, we did not explicitely write the $\sim$ sign over the different hopping amplitudes. Multiplying Eq. (3.13) with the $2 \times 2$-matrix $\left(g_{\sigma \sigma^{\prime}}(z)\right)$ from the left, we obtain a system of quadratic equa- 
tions for the Green's function $g_{\sigma \sigma^{\prime}}(z)$ which is given in matrix notation by

$$
\begin{aligned}
\left(\begin{array}{ll}
0 & 0 \\
0 & 0
\end{array}\right)= & \left(\begin{array}{ll}
1 & 0 \\
0 & 1
\end{array}\right)-\left(\begin{array}{cc}
z+\mu & 0 \\
0 & z+\mu
\end{array}\right)\left(\begin{array}{cc}
g_{\uparrow \uparrow} & g_{\uparrow \downarrow} \\
g_{\downarrow \uparrow} & g_{\downarrow \downarrow}
\end{array}\right) \\
& -\left(\begin{array}{cc}
g_{\uparrow \uparrow} & g_{\uparrow \downarrow} \\
g_{\downarrow \uparrow} & g_{\downarrow \downarrow}
\end{array}\right)\left(\begin{array}{cc}
-t+i \alpha_{y} & -\alpha_{z} \\
\alpha_{z} & -t-i \alpha_{y}
\end{array}\right) \\
& \times\left(\begin{array}{cc}
g_{\uparrow \uparrow} & g_{\uparrow \downarrow} \\
g_{\downarrow \uparrow} & g_{\downarrow \downarrow}
\end{array}\right)\left(\begin{array}{cc}
-t-i \alpha_{y} & -\alpha_{z} \\
\alpha_{z} & -t+i \alpha_{y}
\end{array}\right),
\end{aligned}
$$

using the short-hand notation $g_{\sigma \sigma^{\prime}}$ instead of $g_{\sigma \sigma^{\prime}}(z)$. This matrix equation can be solved by a computer algebra program leading to several solutions all of which are impossible for physical reasons except for Eq. (3.13).

We now give a general proof for the fact that the spin-flip components of the left and right lead's Green's function vanish, i.e. $g_{\uparrow !}^{L, R}=g_{\downarrow \uparrow}^{L, R}=0$. The different hopping terms provided by Eq. (3.12) are visualized in Fig. A.1. Again, we do not explicitely write the ${ }^{\sim}$ sign over the leads' hopping amplitudes for convenience. We can combine

conventional hopping

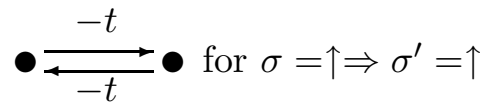

$\bullet \stackrel{-t}{\underset{-t}{\rightleftarrows}} \bullet$ for $\sigma=\downarrow \Rightarrow \sigma^{\prime}=\downarrow$
SOI $\left(\alpha_{y}\right)$ hopping

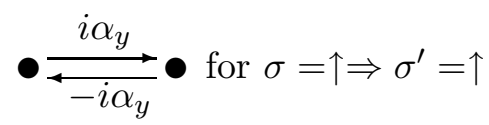
• $\underset{i \alpha_{y}}{\stackrel{-i \alpha_{y}}{\rightleftarrows}} \bullet$ for $\sigma=\downarrow \Rightarrow \sigma^{\prime}=\downarrow$

SOI $\left(\alpha_{z}\right)$ hopping

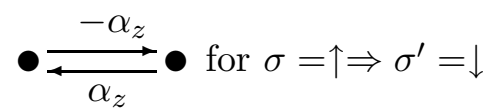

$\bullet \stackrel{\alpha_{z}}{\underset{-\alpha_{z}}{\rightleftarrows}} \bullet$ for $\sigma=\downarrow \Rightarrow \sigma^{\prime}=\uparrow$

Figure A.1: Visualization of the conventional hopping and the SOI hopping.

the conventional hopping and the SOI $\left(\alpha_{y}\right)$ hopping to an effective spin conserving hopping with amplitude $-t \pm i \alpha_{y}$, depending on $\sigma$ and the hopping direction. The spin-flip hopping is given by $\pm \alpha_{z}$.

Let $n \in \mathbb{N}^{\geq 2}$. An arbitrary closed path of $n$th order ( $n$ hopping steps) accomplishes

$$
\begin{aligned}
n & =n_{x}+n_{-x} \\
& =n(\mathrm{sc}, \uparrow \uparrow)+n(\mathrm{sc}, \downarrow \downarrow)+n(\mathrm{sf}, \uparrow \downarrow)+n(\mathrm{sf}, \downarrow \uparrow)
\end{aligned}
$$


and

$$
n_{x}=n_{-x}
$$

with $n_{x}$ and $n_{-x}$ being the number of hopping steps in direction $\pm x$ and $n\left(\mathrm{sc}, \sigma \sigma^{\prime}\right)$, $n\left(\right.$ sf, $\left.\sigma \sigma^{\prime}\right)$ being the number of spin conserving and spin-flip hopping steps, respectively. The first spin index labels the initial state, the second spin index labels the final state after one hopping process. Naturally, the equation

$$
\sum_{\sigma} n(\mathrm{sf}, \sigma-\sigma)=2 m+1 \quad, m=0,1,2, \ldots .
$$

must hold in order to get an effective spin-flip. Following Fig. A.1 the hopping steps of a path $S$ can be decomposed into

$$
n(S)=\underbrace{n_{x}(\mathrm{sc})+n_{x}(\mathrm{sf})}_{n_{x}}+\underbrace{n_{-x}(\mathrm{sc})+n_{-x}(\mathrm{sf})}_{n_{-x}}
$$

and give the contribution

$$
\begin{aligned}
B(S)= & \left(-t+i \alpha_{y}\right)^{n_{x}(\mathrm{sc}, \uparrow \uparrow)}\left(-t-i \alpha_{y}\right)^{n_{x}(\mathrm{sc}, \downarrow \downarrow)} \\
& \times\left(-\alpha_{z}\right)^{n_{x}(\mathrm{sf}, \uparrow \downarrow)}\left(\alpha_{z}\right)^{n_{x}(\mathrm{sf}, \downarrow \uparrow)} \\
& \times\left(-t-i \alpha_{y}\right)^{n_{-x}(\mathrm{sc}, \uparrow \uparrow)}\left(-t+i \alpha_{y}\right)^{n_{-x}(\mathrm{sc}, \downarrow \downarrow)} \\
& \times\left(\alpha_{z}\right)^{n_{-x}(\mathrm{sf}, \uparrow \downarrow)}\left(-\alpha_{z}\right)^{n_{-x}(\mathrm{sf}, \downarrow \uparrow)}
\end{aligned}
$$

to the corresponding Green's function.

For any closed path $S$ the inverse path $\tilde{S}$ exists, which means that all lattice points are passed in reversed order but with the same processes (i.e. conserving or flipping the spin). Note that the total number of spin flips is conserved and regarding to the sequence of different processes, the direction $\sigma \rightarrow \sigma^{\prime}$ of a spin-flip process between each lattice sites remains the same, but, of course, in the opposite spatial direction. The spin conserving hopping processes also happen between the same lattice sites as for the path $S$, but with the opposite spin. Therefore, we obtain

$$
\begin{array}{ll}
\text { spin conserving hopping : } & \tilde{n}_{ \pm x}(\mathrm{sc}, \uparrow \uparrow)=n_{\mp x}(\mathrm{sc}, \downarrow \downarrow) \\
& \tilde{n}_{ \pm x}(\mathrm{sc}, \downarrow \downarrow)=n_{\mp x}(\mathrm{sc}, \uparrow \uparrow) \\
\text { spin-flip hopping : } & \tilde{n}_{ \pm x}(\mathrm{sf}, \uparrow \downarrow)=n_{\mp x}(\mathrm{sf}, \uparrow \downarrow) \\
& \tilde{n}_{ \pm x}(\mathrm{sf}, \downarrow \uparrow)=n_{\mp x}(\mathrm{sf}, \downarrow \uparrow)
\end{array}
$$

Since Eq. A.5 holds for the inverse path as well, we get

$$
\begin{aligned}
B(\tilde{S})= & \left(-t+i \alpha_{y}\right)^{\tilde{n}_{x}(\mathrm{sc}, \uparrow \uparrow)}\left(-t-i \alpha_{y}\right)^{\tilde{n}_{x}(\mathrm{sc}, \downarrow \downarrow)} \\
& \times\left(-\alpha_{z}\right)^{\tilde{n}_{x}(\mathrm{sf}, \uparrow \downarrow)}\left(\alpha_{z}\right)^{\tilde{n}_{x}(\mathrm{sf}, \downarrow \uparrow)} \\
& \times\left(-t-i \alpha_{y}\right)^{\tilde{n}_{-x}(\mathrm{sc}, \uparrow \uparrow)}\left(-t+i \alpha_{y}\right)^{\tilde{n}_{-x}(\mathrm{sc}, \downarrow \downarrow)} \\
& \times\left(\alpha_{z}\right)^{\tilde{n}_{-x}(\mathrm{sf}, \uparrow \downarrow)}\left(-\alpha_{z}\right)^{\tilde{n}_{-x}(\mathrm{sf}, \downarrow \uparrow)} \\
= & (-1)^{n_{x}(\mathrm{sf}, \uparrow \downarrow)+n_{x}(\mathrm{sf}, \downarrow \uparrow)+n_{-x}(\mathrm{sf}, \uparrow \downarrow)+n_{-x}(\mathrm{sf}, \downarrow \uparrow)} B(S) \\
= & (-1)^{2 m+1} B(S)=-B(S) .
\end{aligned}
$$


Therefore, the contribution of any closed path $S$ to the Green's functions $g_{\uparrow \downarrow}^{L, R}$ and $g_{\downarrow \uparrow}^{L, R}$ is cancelled by the contribution of their reversed path $\tilde{S}$ and we obtain

$$
g_{\uparrow \downarrow}^{L, R}=g_{\downarrow \uparrow}^{L, R}=0 .
$$




\section{Appendix B}

\section{$\mathcal{O}(N)$-algorithm for matrix inversion in presence of SOI}

According to Eq. (4.30), calculating the inverse of a large $2 N$-dimensional matrix, i.e. the full propagator $\tilde{\mathcal{G}}^{\Lambda}$, for a system with $N$ lattice sites (factor 2 because of spin) in each step of the numerical integration of the fRG flow equations plays a crucial role with respect to computational effort. A full inversion can be done by an $\mathcal{O}\left(N^{3}\right)$-algorithm, which severely slows down the calculations for large $N$. Even if one neglects the fRG flow of the two-particle vertex, the calculation of a conductance curve for a system with $N=1000$ lattice sites with e.g. 200 data points takes over one day on a single-core pentium 4 processor with $3 \mathrm{GHz}$. However, as we do not account for next-nearest neighbor effects in the hopping or the Coulomb interaction, we only need the tridiagonal part of the full propagator and the self energy for integrating the flow equations. For a conventional Hubbard Model, a short introduction to how this can be done by an $\mathcal{O}(N)$-algorithm was given in Ref. 43 .

\section{B.1 Matrix representation in presence of SOI}

Indeed, things become more complicated in presence of SOI. Having chosen a latticespin base for describing the Hamiltonian results in a matrix representation $\tilde{\mathcal{G}}_{j, j^{\prime}}^{\Lambda}\left(\sigma, \sigma^{\prime}\right)$ and $\Sigma_{j, j^{\prime}}^{\Lambda}\left(\sigma, \sigma^{\prime}\right)$ for the propagator and the self energy. One can interpret this tensor product of lattice and spin space as a conventional lattice representation with matrix elements $A_{j, j^{\prime}}$ being itself complex $2 \times 2$-matrices representing the spin space, i.e.

$$
A_{j, j^{\prime}}=\left(\begin{array}{cc}
A_{j, j^{\prime}}(\uparrow \uparrow) & A_{j, j^{\prime}}(\uparrow \downarrow) \\
A_{j, j^{\prime}}(\downarrow \uparrow) & A_{j, j^{\prime}}(\downarrow \downarrow)
\end{array}\right)
$$

As an illustration, we write down the matrix elements of the inverse of the free propagator being inserted into the Dyson equation [see Eq. (4.30)]. For an arbitrary magnetic field $\mathbf{B}=\left(B_{x}, B_{y}, B_{z}\right)$, chemical potential $\mu$, conventional hopping $t$ and 
SOI components $\alpha_{y}, \alpha_{z}$, the diagonal elements are given by

$$
\left(\mathcal{G}_{j, j}^{0}\right)^{-1}(i \Lambda)=\left(\begin{array}{cc}
i \Lambda+\mu-\gamma B_{z} & \gamma\left(-B_{x}+i B_{y}\right) \\
\gamma\left(-B_{x}-i B_{y}\right) & i \Lambda+\mu+\gamma B_{z}
\end{array}\right)
$$

the upper secondary diagonal elements by

$$
\left(\mathcal{G}_{j, j+1}^{0}\right)^{-1}(i \Lambda)=\left(\begin{array}{cc}
t-i \alpha_{y} & -\alpha_{z} \\
\alpha_{z} & t+i \alpha_{y}
\end{array}\right)
$$

and the lower secondary diagonal elements by

$$
\left(\mathcal{G}_{j+1, j}^{0}\right)^{-1}(i \Lambda)=\left(\begin{array}{cc}
t+i \alpha_{y} & \alpha_{z} \\
-\alpha_{z} & t-i \alpha_{y}
\end{array}\right)
$$

Obviously, the elements of the lower secondary diagonal are the adjoints of the upper secondary diagonal, but the corresponding $2 \times 2$-matrices themselves are not Hermitian. This structure remains conserved, if we subtract the self energy according to Eq. (4.30) with the influence of the leads and one-particle potentials induced by a potential step or other impurities being included in the self energy. Thus, the inverse of the full propagator can be written as

$$
T=\left(\tilde{\mathcal{G}}^{\Lambda}\right)^{-1}=\left(\begin{array}{ccccc}
A_{1} & B_{1} & 0 & \cdots & 0 \\
B_{1}^{\dagger} & A_{2} & \ddots & & \vdots \\
0 & \ddots & \ddots & \ddots & 0 \\
\vdots & & \ddots & \ddots & B_{N-1} \\
0 & \cdots & 0 & B_{N-1}^{\dagger} & A_{N}
\end{array}\right)
$$

with $A_{1}, \ldots, A_{N}$ and $B_{1}, \ldots, B_{N-1}$ being complex $2 \times 2$-matrices. We now use a modification of the standard $L U$-decomposition [51]. We decompose the matrix $T$ into the product of a lower-diagonal matrix $L$, a diagonal matrix $D$ and an upperdiagonal matrix $U$ ( $L D U$-decomposition) via

$$
T=\left(\begin{array}{cccc}
\mathbf{1}_{2} & 0 & & 0 \\
L_{1} & \mathbf{1}_{2} & \ddots & \\
& \ddots & \ddots & 0 \\
0 & & L_{N-1} & \mathbf{1}_{2}
\end{array}\right)\left(\begin{array}{cccc}
D_{1} & 0 & & 0 \\
0 & D_{2} & \ddots & \\
& \ddots & \ddots & 0 \\
0 & & 0 & D_{N}
\end{array}\right)\left(\begin{array}{cccc}
\mathbf{1}_{2} & U_{1} & & 0 \\
0 & \mathbf{1}_{2} & \ddots & \\
& \ddots & \ddots & U_{N-1} \\
0 & & 0 & \mathbf{1}_{2}
\end{array}\right),
$$

with $L_{1}, \ldots, L_{N-1}, D_{1}, \ldots, D_{N}, U_{1}, \ldots, U_{N-1} \in \mathbb{C}^{(2,2)}$. Similarly, we establish an $\tilde{U} \tilde{D} \tilde{L}$ decomposition

$$
T=\tilde{U} \tilde{D} \tilde{L}
$$

with an upper-diagonal matrix $\tilde{U}$, a diagonal matrix $\tilde{D}$ and a lower-diagonal matrix $\tilde{L}$ with $\tilde{U}, \tilde{D}, \tilde{L} \in \mathbb{C}^{(2 N, 2 N)}$. Note that in contrast to the Hubbard model in which $U_{j}=L_{j}$ holds with $U_{j}, L_{j} \in \mathbb{R}$ (see e.g. Ref. [43]), we do not have any further 
symmetry between $U_{j}$ and $L_{j}$, but $L_{j}=U_{j}^{\dagger} D_{j}^{\dagger} D_{j}^{-1} \neq U_{j}^{\dagger}$, since $D_{j}$ is not Hermitian. By a simple comparison of the coefficients in Eqs. (B.6) and (B.7), we get

$$
D_{1}=A_{1}, \quad U_{i}=D_{i}^{-1} B_{i}, \quad D_{i+1}=A_{i+1}-L_{i} B_{i} \quad \text { for } i=1, \ldots, N-1
$$

and

$$
\tilde{D}_{N}=A_{N}, \quad U_{i}=B_{i} \tilde{D}_{i+1}^{-1}, \quad \tilde{D}_{i}=A_{i}-B_{i} \tilde{L}_{i} \quad \text { for } i=1, \ldots, N-1
$$

Thus, we can get all matrix elements of the $L D U$ - and $\tilde{U} \tilde{D} \tilde{L}$-decomposition by successive insertion. Using $T \mathcal{G}=\mathcal{G} T=\mathbf{1}_{2 N}$ with $\mathcal{G}=\left(G_{i, j}\right)$ and $G_{i, j} \in \mathbb{C}^{(2,2)}$ for $i, j=1, \ldots, N$, we obtain from the $L D U$-decomposition by comparison of coefficients

$$
G_{N, N}=D_{N}^{-1}, G_{i, i}=D_{i}^{-1}-U_{i} G_{i+1, i}, G_{i, i+1}=-U_{i} G_{i+1, i+1}, G_{i+1, i}=-G_{i+1, i+1} L_{i}
$$

for $i=1, \ldots, N-1$ and from the $\tilde{U} \tilde{D} \tilde{L}$-decomposition

$$
G_{1,1}=\tilde{D}_{1}^{-1}, \quad G_{i, i}=\tilde{D}_{i}^{-1}-\tilde{L}_{i-1} G_{i-1, i}, \quad G_{i, i+1}=-G_{i, i} \tilde{U}_{i}, \quad G_{i+1, i}=-\tilde{L}_{i} G_{i, i}
$$

for $i=2, \ldots, N$. Finally, we can perform a steplike successive calculation.

$$
\begin{aligned}
& G_{1,1} \quad=\tilde{D}_{1}^{-1} \quad \stackrel{\tilde{U} \tilde{D} \tilde{L}}{\longrightarrow} \quad \begin{array}{lll}
G_{1,2} & =-G_{1,1} \tilde{U}_{1} \\
G_{2,1} & =-\tilde{L}_{1} G_{1,1}
\end{array} \stackrel{L D U}{\longrightarrow} G_{2,2} \quad=-U_{1}^{-1} G_{12} \\
& G_{2,2} \quad=-U_{1}^{-1} G_{12} \stackrel{\tilde{U} \tilde{D} \tilde{L}}{\longrightarrow} \begin{array}{l}
G_{2,3}=-G_{2,2} \tilde{U}_{2} \\
G_{3,2}=-\tilde{L}_{2} G_{2,2}
\end{array} \stackrel{L D U}{\longrightarrow} G_{3,3} \quad=-U_{2}^{-1} G_{23} \\
& G_{N-1, N-1}=\cdots \quad \stackrel{\tilde{U} \tilde{D} \tilde{L}}{\longrightarrow} \quad \ldots \quad \stackrel{L D U}{\longrightarrow} \quad \ldots \quad \Rightarrow \text { finished }
\end{aligned}
$$

In this way, we obtain all tridiagonal, i.e. diagonal and upper/lower secondary diagonal, matrix elements $G_{i j}$ of the full propagator $\tilde{\mathcal{G}}$ from the $L D U$ - and $\tilde{U} \tilde{D} \tilde{L}$ decomposition in $N$ calculation steps. Furthermore, the numerical effort for performing the $L D U$ - and $\tilde{U} \tilde{D} \tilde{L}$-decomposition according to Eqs. (B.8) and (B.9) is of $\mathcal{O}(N)$. Thus, the complete algorithm is of $\mathcal{O}(N)$ as well.

Care must be taken in the case of matrices which are invertible but not $L D U$ (or $\tilde{U} \tilde{D} \tilde{L}-$ ) decomposable. Naturally, such matrices exist. Indeed, sometimes we have suffered from numerical problems in presence of SOI and magnetic field for very large systems, but this was due to imprecise intermediate data during the integration of the fRG flow equations rather than an immanent problem of the method. However, varying the system parameters slightly and increasing the accuracy of the numerical integration of the flow equations, we got rid of these problems, at least in the majority of cases. 


\section{B.2 Calculation of the $G_{1, N}$ matrix element of the full propagator}

As clarified in Eqs. (3.22) and (3.23), the matrix element $G_{1, N}$ of the full propagator at the end of the fRG flow is essential in order to calculate the linear conductance. Certainly, $G_{1, N}$ can not be calculated in the scheme mentioned above, but it is not neccessary to perform a full $\mathcal{O}\left(N^{3}\right)$-inversion. Having obtained the self energy $\Sigma^{\Lambda=0}$ at the end of the flow, we set up the inverse $T$ of the full propagator up according to Eq. (4.30) again and perform a $L D U$-decomposition according to Eq. (B.6). Defining new matrices $P=L$ and $Q=D U$, we obtain

$$
T=P Q=\left(\begin{array}{cccc}
\mathbf{1}_{2} & 0 & & 0 \\
L_{1} & \mathbf{1}_{2} & \ddots & \\
& \ddots & \ddots & 0 \\
0 & & L_{N-1} & \mathbf{1}_{2}
\end{array}\right)\left(\begin{array}{cccc}
D_{1} & D_{1} U_{1} & & 0 \\
0 & D_{2} & \ddots & \\
& \ddots & \ddots & D_{N-1} U_{N-1} \\
0 & & 0 & D_{N}
\end{array}\right)
$$

Generally, the inverse matrices $V=\left(V_{i, j}\right)=P^{-1}$ and $W=\left(W_{i, j}\right)=Q^{-1}$ are full lower and upper triangular matrices, respectively. Using $V P=\mathbf{1}_{2 N}$ and $W Q=\mathbf{1}_{2 N}$, we get

$$
\begin{aligned}
& V_{i, j}=\quad 0 \quad \text { for } i<j \\
& V_{i, j}=\quad \mathbf{1}_{2} \quad \text { for } i=j \\
& V_{i, j}=-L_{i-1} V_{i-1, j} \quad \text { for } \quad i>j \\
& W_{i, j}=-U_{i} W_{i+1, j} \quad \text { for } \quad i<j \\
& W_{i, j}=D_{i}^{-1} \quad \text { for } i=j \\
& W_{i, j}=\quad 0 \quad \text { for } i>j
\end{aligned}
$$

with $V_{i, j}, W_{i, j} \in \mathbb{C}^{(2,2)}$ for $i, j=1, \ldots, N$.

Inverting the equation $T=P Q$ leads to

$$
\tilde{\mathcal{G}}=T^{-1}=Q^{-1} P^{-1}=W V
$$

and especially

$$
G_{1, N}=\sum_{i=1}^{N} W_{1, i} V_{i, N}=W_{1, N} V_{N, N}
$$


since $V_{i, j}=0$ for $i<j$ according to Eq. (B.13). We further obtain

$$
\begin{aligned}
G_{1, N}= & W_{1, N} V_{N, N}=W_{1, N} \\
= & -U_{1} W_{2, N} \\
= & \left(-U_{1}\right)\left(-U_{2}\right) W_{3, N} \\
& \vdots \\
= & \left(-U_{1}\right) \cdots\left(-U_{N-1}\right) W_{N, N}=\left(-U_{1}\right) \cdots\left(-U_{N-1}\right) D_{N}^{-1} \\
= & {\left[\prod_{i=1}^{N-1}\left(-U_{i}\right)\right] D_{N}^{-1} . }
\end{aligned}
$$

Thus, we can calculate $G_{1, N}$ in a single loop from the $L D U$-decomposition of $T$.

The algorithms presented in this chapter are very similar to those used for the conventional Hubbard model, despite the fact that we have to deal with an algebra of complex $2 \times 2$-matrices and not just complex (or even real) numbers. Taking into account this matrix algebra, one can use more or less the same routines for the calculations. 


\section{Appendix C}

\section{The eigenvalue problem of the double dot}

The Hamiltonian describing the double dot with SOI and magnetic field can be written as a matrix in lattice-spin representation leading to

$$
\bar{H}=\left(\begin{array}{cccc}
\mu+\gamma B_{z} & \gamma\left(B_{x}-i B_{y}\right) & -t+i \alpha_{y} & \alpha_{z} \\
\gamma\left(B_{x}+i B_{y}\right) & \mu-\gamma B_{z} & -\alpha_{z} & -t-i \alpha_{y} \\
-t-i \alpha_{y} & -\alpha_{z} & \mu+\gamma B_{z} & \gamma\left(B_{x}-i B_{y}\right) \\
\alpha_{z} & -t+i \alpha_{y} & \gamma\left(B_{x}+i B_{y}\right) & \mu-\gamma B_{z}
\end{array}\right)
$$

The eigenvalues and eigenvectors in this most general case can easily be obtained by a computer algebra program, but are quite cumbersome. We therefore concentrate on the situation given in Fig. 6.2 where $B_{x}=0, B_{y}=0$ and $\alpha_{y}=0$. In this case, we obtain the four eigenvalues

$$
\begin{aligned}
& \lambda_{1}=\mu+\delta_{1} \\
& \lambda_{2}=\mu+\delta_{2} \\
& \lambda_{3}=\mu-\delta_{2} \\
& \lambda_{4}=\mu-\delta_{1}
\end{aligned}
$$

with

$$
\begin{aligned}
& \delta_{1}=\sqrt{\alpha_{z}^{2}+\left(t+\gamma B_{z}\right)^{2}} \\
& \delta_{2}=\sqrt{\alpha_{z}^{2}+\left(t-\gamma B_{z}\right)^{2}} .
\end{aligned}
$$


The normalized eigenvectors are given by

$$
\begin{aligned}
& \mathbf{a}_{1}=\frac{1}{N_{1}}\left(\begin{array}{c}
\frac{t+\gamma B_{z}+\delta_{1}}{\alpha_{z}} \\
1 \\
\frac{-t-\gamma B_{z}-\delta_{1}}{\alpha_{z}} \\
1
\end{array}\right) \quad \text { with } \quad N_{1}=\left(2+\frac{\left(t+\gamma B_{z}+\delta_{1}\right)^{2}}{\alpha_{z}^{2}}\right)^{1 / 2} \\
& \mathbf{a}_{2}=\frac{1}{N_{2}}\left(\begin{array}{c}
\frac{-t+\gamma B_{z}+\delta_{2}}{\alpha_{z}} \\
\frac{-t+\gamma B_{z}+\delta_{2}}{\alpha_{z}} \\
1
\end{array}\right) \quad \text { with } \quad N_{2}=\left(2+\frac{\left(t-\gamma B_{z}-\delta_{2}\right)^{2}}{\alpha_{z}^{2}}\right)^{1 / 2} \\
& \mathbf{a}_{3}=\frac{1}{N_{3}}\left(\begin{array}{c}
\frac{-t+\gamma B_{z}-\delta_{2}}{\alpha_{z}} \\
-1 \\
\frac{-t+\gamma B_{z}-\delta_{2}}{\alpha_{z}} \\
1
\end{array}\right) \quad \text { with } \quad N_{3}=\left(2+\frac{\left(t-\gamma B_{z}+\delta_{2}\right)^{2}}{\alpha_{z}^{2}}\right)^{1 / 2} \\
& \mathbf{a}_{4}=\frac{1}{N_{4}}\left(\begin{array}{c}
\frac{t+\gamma B_{z}-\delta_{1}}{\alpha_{z}} \\
1 \\
\frac{-t-\gamma B_{z}+\delta_{1}}{\alpha_{z}} \\
1
\end{array}\right) \quad \text { with } \quad N_{4}=\left(2+\frac{\left(t+\gamma B_{z}-\delta_{1}\right)^{2}}{\alpha_{z}^{2}}\right)^{1 / 2}
\end{aligned}
$$

The matrix $\mathcal{M}=\left(\mathbf{a}_{1}, \mathbf{a}_{2}, \mathbf{a}_{3}, \mathbf{a}_{4}\right)$ built of these eigenvectors is just the transformation matrix from the lattice-spin representation to the energy representation of our model. We can therefore transform the Hamiltonian Eq. (3.12) describing the contact between the dot and the leads in order to determine the coupling of the four energy levels to the leads. It is shown in Fig. 6.3 that electrons with different spin $\sigma=\uparrow$ couple to the energy levels in a way different from electrons with $\sigma=\downarrow$. Now we would like to address the question how the energy levels are coupled to each other. We therefore analyze the influence of the hopping terms $t$ and $\alpha_{z}$ on the transformed expressions $c_{i}^{\dagger} c_{j}$ with $i, j \in 1, \ldots, 4$ and $c_{i}^{\dagger}\left(c_{i}\right)$ denoting the creation (annihilation) operator of the electrons on energy level $\lambda_{i}$. This is shown in Table C.1.

The two outer levels $\lambda_{1}=\mu+\delta_{1}$ and $\lambda_{4}=\mu-\delta_{1}$ are coupled to each other via hopping processes induced by $t$ and $\alpha_{z}$. The same holds for the inner levels $\lambda_{2}=\mu+\delta_{2}$ and $\lambda_{3}=\mu-\delta_{2}$. Interestingly, there is no coupling between inner and outer levels. 


\begin{tabular}{c|c|c} 
operator & contribution induced by $t$ & contribution induced by $\alpha_{z}$ \\
\hline$c_{1}^{\dagger} c_{1}$ & $\frac{-2 t\left(\alpha_{z}^{2}-\left(\delta_{1}+\gamma B_{z}+t\right)^{2}\right)}{\alpha_{z}^{2} N_{1}^{2}}$ & $\frac{4\left(\delta_{1}+\gamma B_{z}+t\right)}{N_{1}^{2}}$ \\
$c_{1}^{\dagger} c_{2}$ & 0 & 0 \\
$c_{1}^{\dagger} c_{3}$ & 0 & 0 \\
$c_{1}^{\dagger} c_{4}$ & $\frac{-4 t}{N_{1} N_{4}}$ & $\frac{4\left(\gamma B_{z}+t\right)}{N_{1} N_{4}}$ \\
$c_{2}^{\dagger} c_{2}$ & $\frac{2 t\left(\alpha^{2}-\left(\delta_{2}+\gamma B_{z}-t\right)^{2}\right)}{\alpha_{z}^{2} N_{2}^{2}}$ & $\frac{4\left(\delta_{2}+\gamma B_{z}-t\right)}{N_{2}^{2}}$ \\
$c_{2}^{\dagger} c_{3}$ & $\frac{4 t}{N_{2} N_{3}}$ & $\frac{4\left(\gamma B_{z}-t\right)}{N_{2} N_{3}}$ \\
$c_{2}^{\dagger} c_{4}$ & 0 & 0 \\
$c_{3}^{\dagger} c_{3}$ & $\frac{2 t\left(\alpha_{z}^{2}-\left(\delta_{2}-\gamma B_{z}+t\right)^{2}\right)}{\alpha_{z}^{2} N_{3}^{2}}$ & $\frac{4\left(-\delta_{2}+\gamma B_{z}-t\right)}{N_{3}^{2}}$ \\
$c_{3}^{\dagger} c_{4}$ & 0 & 0 \\
$c_{4}^{\dagger} c_{4}$ & $\frac{-2 t\left(\alpha_{z}^{2}-\left(\delta_{1}-\gamma B_{z}-t\right)^{2}\right)}{\alpha_{z}^{2} N_{4}^{2}}$ & $\frac{4\left(-\delta_{1}+\gamma B_{z}+t\right)}{N_{4}^{2}}$
\end{tabular}

Table C.1: Contributions to the operators $c_{i}^{\dagger} c_{j}$ induced by $t$ and $\alpha_{z} \cdot c_{i}^{\dagger}\left(c_{i}\right)$ denotes the creation (annihilation) operator of an electron with energy $\lambda_{i}$. 


\section{Bibliography}

[1] M.N. Baibich, J.M. Broto, A. Fert, F. Nguyen van Dau, F. Petroff, P. Etienne, G. Creuzet, A. Friederich, and J. Chazelas, Phys. Rev. Lett. 61, 2472 (1988).

[2] G. Binasch, P. Grünberg, F. Saurenbach, and W. Zinn, Phys. Rev. B 39, 4828(R) (1989).

[3] S. Datta and B. Das, Appl. Phys. Lett. 56, 7 (1990).

[4] J.C. Egues, C. Gould, G. Richter, and L.W. Mohlenkamp, Phys. Rev. B 64, 195319 (2001).

[5] C.W.J. Beenakker, Phys. Rev. B 73, 201304 (2006).

[6] S.A. Wolf, D.D. Awschalom, R.A. Buhrman, J.M. Daughton, S. von Molnár, M.L. Roukes, A.Y. Chtchelkanova, and D.M. Treger, Science 294, 1488 (2001).

[7] K. G. Wilson, Rev. Mod. Phys. 47, 773 (1975).

[8] M. Salmhofer, Renormalization, Springer-Verlag, Berlin, 1999.

[9] V. Meden, lecture notes on the "Functional renormalization group", http://www.theorie.physik.uni-goettingen.de/ meden/funRG/

[10] For a review on the NRG method, see R. Bulla, T.A. Costi, and T. Pruschke, Rev. Mod. Phys. 80, 395 (2008).

[11] S.R. White, Phys. Rev. Lett. 69, 2863 (1992).

[12] S.R. White, Phys. Rev. B 48, 10345 (1993).

[13] For a review on the DMRG method, see U. Schollwöck, Rev. Mod. Phys. 77, 259 (2005).

[14] J.M. Luttinger, J. Math. Phys. 4, 1154 (1963).

[15] T. Giamarchi, Quantum physics in One Dimension, Oxford University Press, New York, 2004.

[16] V. Meden, S. Andergassen, W. Metzner, U. Schollwöck, and K. Schönhammer, Europhys. Lett. 64, 769 (2003). 
[17] T. Enss, V. Meden, S. Andergassen, X. Barnabé-Thériault, W. Metzner, and K. Schönhammer, Phys. Rev. B 71, 155401 (2005).

[18] S. Andergassen, T. Enss, V. Meden, W. Metzner, U. Schollwöck, and K. Schönhammer, Phys. Rev. B 73, 045125 (2006)

[19] J. Kondo, Prog. Theor. Phys. 32, 37 (1964).

[20] C. Karrasch, T. Enss, and V. Meden, Phys. Rev. B 73, 235337 (2006).

[21] R. Winkler, Spin-Orbit Coupling Effects in Two-Dimensional Electron and Hole Systems, Springer-Verlag, Berlin (2003).

[22] F. Kuemmeth, S. Ilani, D.C. Ralph, and P.L. McEuen, Nature 452, 448 (2008).

[23] J.A. Folk, S.R. Patel, K.M. Birnbaum, C.M. Marcus, C.I.Duruöz, and J.S. Harris, Jr., Phys. Rev. Lett. 86, 2102 (2001).

[24] B.I. Halperin, A. Stern, Y. Oreg, J.N.H.J. Cremers, J.A. Fok, and C.M. Marcus, Phys. Rev. Lett. 86, 2106 (2001).

[25] J. J. Sakurai, Advanced Quantum Mechanics, Addison-Wesley, Reading, 1967.

[26] P. Strěda, P. Sěba, Phys. Rev. Lett. 90, 256601 (2003).

[27] A.V. Moroz and C.H.W. Barnes, Phys. Rev. B 60, 14272 (1999).

[28] F. Mireles and G. Kirczenow, Phys. Rev. B 64, 24426 (2001).

[29] M. Governale and U. Zülicke 2002, Phys. Rev. B 66, 07331 (2002); Solid State Com. 131581 (2004).

[30] R. G. Pereira and E. Miranda, Phys. Rev. B 71, 085318 (2005).

[31] L. Serra, D. Sánchez, and R. López, Phys. Rev. B 72, 235309 (2005).

[32] S. Zhang, R. Liang, E. Zhang, L. Zhang, and Y. Liu, Phys. Rev. B 73, 155316 (2006).

[33] C. Dekker, Physics Today 52, no. 5, 22 (1999).

[34] E.I. Rashba, Physica E 34, 31 (2006).

[35] J.E. Birkholz and V. Meden, J. Phys.: Condens. Matter 20, 085226 (2008).

[36] L.W. Molenkamp, G. Schmidt, and G.E.W. Bauer, Phys. Rev. B 64, 121202 (2001).

[37] E.N. Economou, Green's Functions in Quantum Physics, Springer-Verlag, Berlin, (1979). 
[38] Y. Meir and N.S. Wingreen, Phys. Rev. B 50, 4947 (1994).

[39] K. Janzen, V. Meden, and K. Schönhammer, Phys. Rev. B 74, 085301 (2006).

[40] T. Enss Dissertation, Stuttgart (2005).

[41] For a review on the Landauer-Büttiker approach to transport and the Kubo formalism see: S. Datta, Electronic Transport in Mesoscopic Systems, Cambridge University Press, Cambridge, 1995.

[42] H. Bruus and K. Flensberg, Many-body Quantum Theory in Condensed Matter Physics, Oxford University Press, Oxford, 2004.

[43] S. Andergassen, T. Enss, V. Meden, W. Metzner, U. Schollwöck, and K. Schönhammer, Phys. Rev. B 70, 075102 (2004).

[44] I. Lizuain, J.G. Muga, and A. Ruschhaupt, Phys. Rev. A 74, 053608 (2006).

[45] S.J. Tans, M.H. Devoret, H. Dai, A. Thess, R.E. Smalley, L.J. Geerligs, and C. Dekker, Nature 386, 474 (1997).

[46] K. Wilson, Phys. Rev. D 3, 1818 (1971).

[47] R. Hedden, V. Meden, T. Pruschke, and K.Schönhammer, J. Phys.: Condens. Matter 16, 5279 (2004).

[48] J. W. Negele and H. Orland, Quantum Many-Particle physics, (Addison-Wesley, Reading, 1988).

[49] P. Wächter, V. Meden, and K. Schönhammer, Phys. Rev. B 76, 045123 (2007).

[50] C. Karrasch, Diplomarbeit, Göttingen (2006).

[51] W.H. Press, B.P. Flannery, S.A. Teukolsky, and W.T. Vetterling, Numerical Recipes in C, Cambridge University Press, Cambridge, 1991.

[52] For a review, see J. Voit, Rep. Prog. Phys. 57, 977 (1994).

[53] K. Schönhammer, V. Meden, W. Metzner, U. Schollwöck, and O. Gunnarson, Phys. Rev. B 61, 7 (2000).

[54] For a review, see J. Sólyom, Adv. Phys. 28, 201 (1979).

[55] D.C. Mattis and E.H. Lieb, J. Math. Phys. 6, 304 (1965).

[56] S. Tomonaga, Prog. Theor. Phys. 5, 544 (1950).

[57] For a recent review see K. Schönhamme, Interacting Electrons in Low Dimensions, Ed.: D. Baeriswyl, Kluwer Academic Publishers (2005).

[58] F.D.M. Haldane, J. Phys. C 14, 2585 (1981). 
[59] C.L. Kane and M.P.A. Fisher, Phys. Rev. Lett. 68, 1220 (1992).

[60] C.L. Kane and M.P.A. Fisher, Phys. Rev. B 46, 7268 (1992).

[61] C.L. Kane and M.P.A. Fisher, Phys. Rev. B 46, 15233 (1992).

[62] A. Furusaki and N. Nagaosa, Phys. Rev. B 47, 4631 (1993).

[63] V. Meden, W. Metzner, U. Schollwöck, O. Schneider, T. Stauber, and K. Schönhammer, Eur. Phys. J. B 16, 631 (2000).

[64] S. Andergassen Dissertation, Stuttgart (2005).

[65] For a review, see F. Gebhard, The Mott Metal-Insulator Transition - Models and Methods, Springer-Verlag, Heidelberg, 1997.

[66] D.F. Urban and A. Komnik, Phys. Rev. Lett. 100, 146602 (2008).

[67] D. Loss and D.P. DiVincenzo, Phys. Rev. A 57, 120 (1998).

[68] D. Goldhaber-Gordon, H. Shtrikman, D. Mahalu, D. Abusch-Magder, U. Meirav, and M.A. Kastner, Nature 391, 156 (1998).

[69] Y. Meir and N.S. Wingreen, Phys. Rev. Lett. 68, 2512 (1992).

[70] A.C. Hewson, The Kondo Problem to Heavy Fermions, (Cambridge University Press, Cambridge, UK, 1993).

[71] J. Malecki, J. Stat. Phys. 129, 741 (2007).

[72] T.S. Jespersen, M. Aagesen, C. Sørensen, P.E. Lindelof, and J. Nygård, Phys. Rev. B 74, 233304 (2006).

[73] J.R. Schrieffer and P.A. Wolff, Phys. Rev. 149, 491 (1966).

[74] Q. Sun, J. Wang, and H. Guo, Phys. Rev. B 71, 165310 (2005).

[75] U. Fano, Phys. Rev. 124, 1866 (1961).

[76] R. Whiddington and M. Priestley, Proc. R. Soc. A 145, 462 (1934).

[77] D. Sánchez and L. Serra, Phys. Rev. B 74, 153313 (2006).

[78] A.C. Johnson, J.R. Petta, J.M. Taylor, A. Yacoby, M.D. Lukin, C.M. Marcus, M.P. Hanson, and A.C. Gossard, Nature 435, 925 (2005).

[79] J.R. Petta, A.C. Johnson, J.M. Taylor, E.A. Laird, A. Yacoby, M.D. Lukin, C.M. Marcus, M.P. Hanson, and A.C. Gossard, Science 309, 2180 (1998).

[80] N.J. Craig, J.M. Taylor, E.A. Lester, C.M. Marcus, M.P. Hanson, A.C. Gossard, Science 304, 565 (2004). 
[81] D. Chang, G.L. Khym, K. Kang, Y. Cung, H. Lee, M. Seo, M. Heiblum, D. Mahalu, and V. Umansky, Nature 4, 205 (2008).

[82] G. Schedelbeck, W. Wegschneider, M. Bichler, and G. Abstreiter, Science 278, 1792 (1997).

[83] L.V. Titova, G. Cywiński, M. Kutrowski, T. Wojtowicz, X. Liu, J.K. Furdyna, and M. Dobrowolska, Phys. Stat. Sol. (b) 241, 519 (2004).

[84] S.J. Tans, M.H. Devoret, R.J.A. Groeneveld, and C. Dekker, Nature 394, 761, (1998).

[85] see for example: G. Czycholl, Theoretische Festkörperphysik, (Vieweg, Braunschweig/Düsseldorf, 2000).

[86] I. Schnell, G. Czycholl, and R.C. Albers, Phys. Rev. B 65, 075103 (2002).

[87] B.N. Cox, M.A. Coulthard, and P. Lloyd, J. Phys. F 4, 807 (1974).

[88] O. Pankratov and M. Scheffler, Phys. Rev. Lett. 70, 351 (1993).

[89] D. Grundler, Phys. Rev. Lett. 84, 6074 (2000).

[90] J. Nitta, T. Akazaki, H. Takayanagi, and T. Enoki, Phys. Rev. Lett. 78, 1335 (1997).

[91] G. Engels, J. Lange, T. Schäpers, and H. Lüth, Phys. Rev. B 55, R1958 (1997).

[92] Y. Sato, T. Kita, S. Gozu, and S. Yamada, J. Appl. Phys. 89, 8017 (2001). 


\section{Acknowledgement}

There are a lot of people, who supported me during the last few years in the most different ways so that this thesis finally came to a successful end.

First of all, I want to thank my supervisor Prof. Dr. Volker Meden for giving me the opportunity to write this $\mathrm{PhD}$ thesis in his group and for taking his time, whenever I was approaching him with questions. Especially in the last phase of this thesis, I benefited from his hints and suggestions.

I'm grateful to Prof. Dr. Reiner Kree for agreeing to do the second review. I also thank Dr. Andreas Honecker who often helped me regarding computer problems, and Prof. Dr. Kurt Schönhammer for the interesting lectures on Electrons in low dimensions and Renormalization group methods.

Special thanks go to Steffi Ileona Birkholz, Peter Wächter, Robert Peters and Christoph Karrasch for critically reading the manuscript and furthermore to Christoph for sharing the office with me for the past three years. It was a great pleasure to talk about topics far away from physics as well.

I am grateful to all members of the Institut für Theoretische Physik of the University of Göttingen for a pleasant working environment.

Last but not least, I want to exceptionally thank my family and all of my friends, who calmed me down, built me up and motivated me, whenever I was unhappy with my results and who showed me again and again that there is more in my life than just physics. 


\section{Lebenslauf}

\section{Persönliches}

\begin{tabular}{|c|c|}
\hline Name & Jens Eiko Birkholz \\
\hline Adresse & Stumpfe Eiche $4 \mathrm{a}$ \\
\hline & 37077 Göttingen \\
\hline Tel. & $0176 / 61566287$ \\
\hline email & birkholz@theorie.physik.uni-goettingen.de \\
\hline Geburtsdatum & 10. April 1979 \\
\hline Geburtsort & Hannover \\
\hline Natiol & deutsch \\
\hline
\end{tabular}

\section{Ausbildung}

Jul 1998

Jul 1998 - Jul 1999

Okt 1999 - Jan 2005

Jan 2004 - Jan 2005

Jan 2005

seit Apr 2005
Abitur (Kaiser-Wilhelm-und Ratsgymnasium Hannover), Gesamtnote: 1.1

Zivildienst (Kreiskrankenhaus Lehrte)

Physikstudium an der Universität Hannover und der University of New South Wales, Sydney (Australien)

Diplomarbeit am

Institut für Solarenergieforschung Hameln (ISFH) über

"Eisen-korrelierte Photodegradation in monound multikristallinem Silizium"

Diplom in Physik (Universität Hannover), Gesamtnote: 1.15 (sehr gut)

Promotion bei Prof. Dr. Volker Meden an der Georg-August-Universität Göttingen über

"Spin-orbit interaction in quantum dots and quantum wires of correlated electrons - A way to spintronics?"

Göttingen, den 2.9.2008 